\title{
Research Commons
}

http://researchcommons.waikato.ac.nz/

\section{Research Commons at the University of Waikato}

\section{Copyright Statement:}

The digital copy of this thesis is protected by the Copyright Act 1994 (New Zealand).

The thesis may be consulted by you, provided you comply with the provisions of the Act and the following conditions of use:

- Any use you make of these documents or images must be for research or private study purposes only, and you may not make them available to any other person.

- Authors control the copyright of their thesis. You will recognise the author's right to be identified as the author of the thesis, and due acknowledgement will be made to the author where appropriate.

- You will obtain the author's permission before publishing any material from the thesis. 


\title{
Relationship of the cricothyroid space with vocal range in female singers
}

\author{
A thesis \\ submitted in fulfilment \\ of the requirements for the degree \\ of \\ Doctor of Musical Arts \\ at \\ The University of Waikato \\ by \\ Beverley Pullon
}

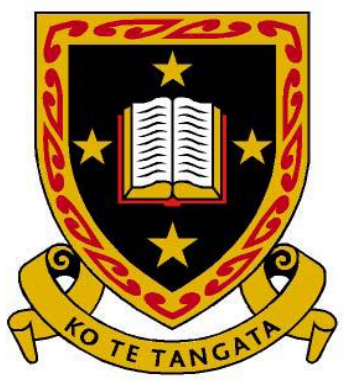

THE UNIVERSITY OF

WAIKATO

Te Whare Wananga o Waikato

2014 


\section{Abstract}

It is well documented that the cricothyroid (CT) space opens and closes with changes in pitch, narrowing with rising pitch and widening with falling pitch. Indeed, cricothyroid approximation surgery, a procedure where the CT space is deliberately made smaller, is used in male to female transgender subjects to successfully elevate vocal pitch.

The present study focuses on investigating the relationship between the anterior CT space at rest and vocal range in female singers. Laryngeal dimensions (anterior CT space and heights of the thyroid and cricoid cartilages) were measured using ultrasound in 43 healthy, classically trained, female singers. Potential associations with and between age, ethnicity, anthropometric indices (height, weight, body mass index), neck dimensions (circumference and length), vocal data (practice and performance vocal range, lowest and highest practice and performance notes) along with usual speaking fundamental frequency were also explored.

The main finding was that mezzo-sopranos have a significantly wider resting CT space than sopranos (11.6 mm versus $10.4 \mathrm{~mm} ; \mathrm{P}=0.007$ ). Mezzo-sopranos also had significantly lower 'lowest and highest' performance notes and speaking fundamental frequencies than sopranos. Furthermore, there was a weak but significant negative correlation between the magnitude of the anterior CT space and the lowest performance note $(r=-0.448 ; P=0.003)$ but there was no significant correlation with either the highest performance note or vocal range.

These results suggest there is a relationship between the CT space and the lowest note a female can sing. This was evident in the correlation of a small CT space with a higher 'lowest performance note'. It appears that the CT space influences how low female singers can sing, but not how high they can sing. 


\section{Acknowledgements}

This research project would not have been possible without the support of many people. I wish to express gratitude to my supervisor, Professor Mark Stringer who was abundantly helpful. Without his knowledge, invaluable assistance, support and guidance, this thesis would never have been completed. I would also like to express appreciation to my chief supervisor Associate Professor Martin Lodge for the useful comments and remarks, and who guided me through this project from the very beginning to the end. Furthermore, I would like to thank Mr David Griffiths and Dr. Rachael Griffiths-Hughes for their support on the way. Deepest gratitude is also due to Dame Malvina Major for voice tuition and guidance in repertoire. To the rest of the Conservatorium of Music staff at University of Waikato who offered support along the way, I am intensely appreciative.

Special acknowledgments also go to Mr Martin Necas for allowing use of the ultrasound facilities at Waikato Hospital, and a heartfelt thanks to Mrs Wendy Wackrow for her expertise and professional manner and making herself available to ultrasound scan every subject.

I would also like to convey thanks to the Post Graduate Department and Hillary Scholarship programme at University of Waikato for providing the financial means.

To the superb library staff Jenny McGhee, Heather Morrell and Cheryl Ward for their unfailing help with references and all aspects of thesis layout. Additionally, to the interloan library staff for managing to procure every obscure journal article, book, compact disc, long playing record and thesis I required, frequently in multiple languages, I am greatly indebted.

For assisting me with statistical analysis I am grateful to Dr. Ray Littler and Dr. Chris Morris. 
I would further like to thank Kayla Collingwood for proof reading and correcting my innumerable grammatical errors.

I am further indebted to my friends and loved ones for their great support and encouragement throughout the entire process. To my longsuffering family, especially my husband Humphrey, who has had to endure many months with tears of frustration. To my daughter Rebecca, who has continually encouraged me to finish my thesis whilst she is currently undertaking a Doctor of Philosophy at Oxford University. Also to my twin sons, Jonathan and Andrew, who had to respond to my frequent SOS calls for technological help. To my parents, Hector and Joy Anderson, who are my biggest fans and supported me by attending every opera, recital and concert I was performing in. I will be grateful forever for your love.

Lastly, I would like to say a special thanks to the participants in my survey, who have willingly shared their precious time during the process of interviewing and testing.

Finally, a big thank you my friends at University of Waikato, Waikato Hospital and elsewhere for their support and encouragement throughout.

For any errors or inadequacies that may remain in this work, the responsibility is entirely my own. 


\section{Table of Contents}

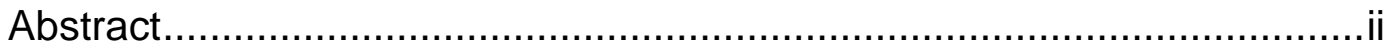

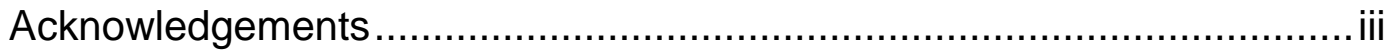

List of Figures ................................................................................ viii

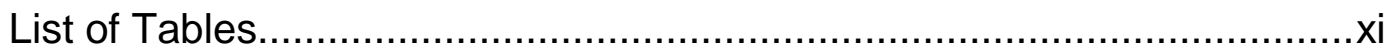

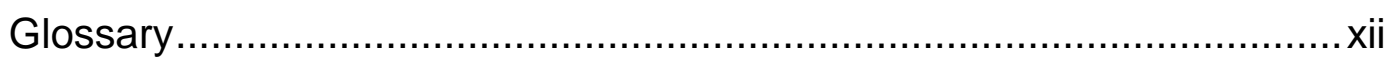

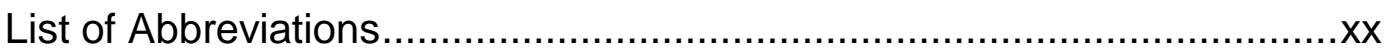

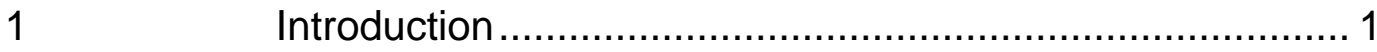

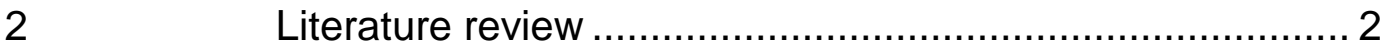

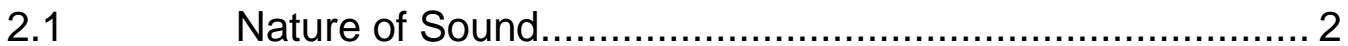

2.1.1 A musical sound wave .................................................... 3

2.1.2 Characteristics of a regular sound wave .......................... 3

2.1.3 How the larynx makes a sound........................................ 5

2.1.4 Sound Spectrogram................................................... 13

2.2 Speaking fundamental frequency ................................... 15

2.2.1 Surgical alteration of fundamental frequency................... 21

2.3 Vocal range and voice classification (Type)..................... 24

2.3.1 Senescence and vocal range ....................................... 26

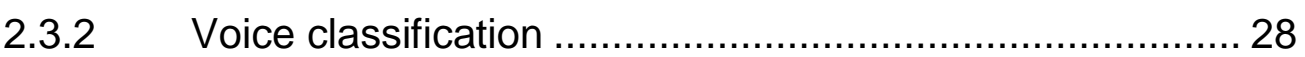

2.3.3 Relationship between voice type and anatomical features 30

2.3.4 Importance of vocal range and voice classification........... 33

2.4 The 'gifted voice' and genetic aspects ............................ 34

$2.5 \quad$ Anatomy of the larynx ........................................................... 36

2.5.1 Laryngeal Cartilages .................................................... 36

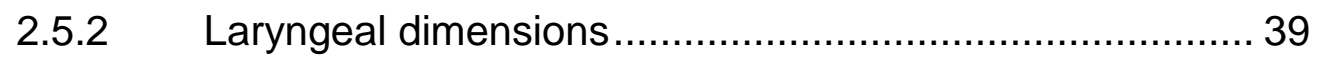

2.5.3 Larynx: Internal anatomy ............................................... 44

2.5.4 Muscles of the Larynx................................................ 46

2.5.5 Cricothyroid joint ..................................................... 50

2.5.6 Muscular control of fundamental frequency ….................. 52

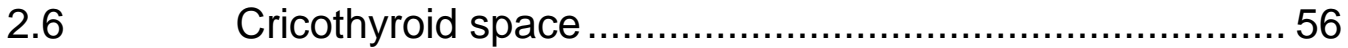

2.6.1 Vocal pitch and the cricothyroid space .......................... 57

2.6.2 Cadaver studies on the cricothyroid space ...................... 58

2.6.3 Ultrasound studies of the cricothyroid space ................... 59

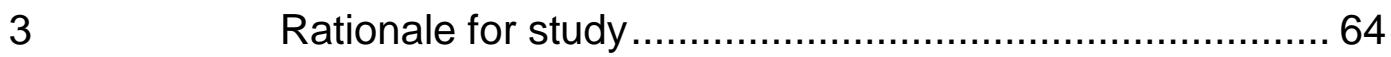




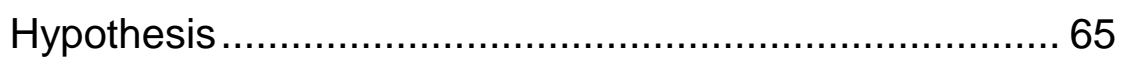

Study Aims ...................................................... 65

5

Materials and Methods ............................................. 66

5.1 Participants ........................................................... 66

Equipment and Measurements ..................................66 66

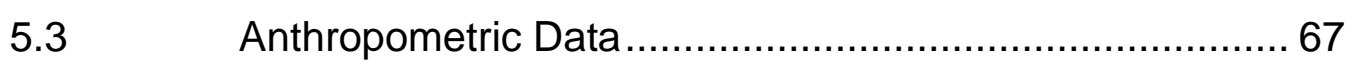

$5.4 \quad$ Neck Dimensions................................................. 68

$5.5 \quad$ Vocal Data .......................................................... 71

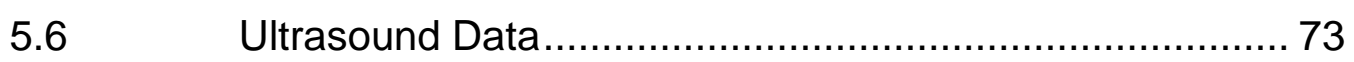

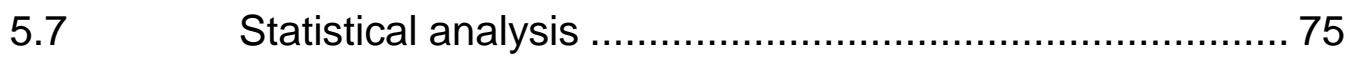

Ethical Approval.............................................. 76

$6 \quad$ Results............................................................. 77

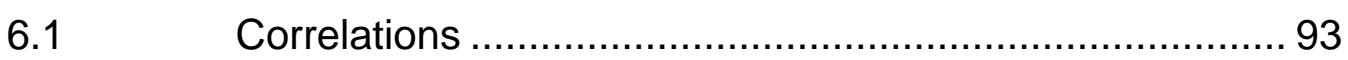

6.1.1 CT space correlations ........................................ 93

6.1.2 Speaking fundamental frequency correlations ............... 100

6.1.3 Other performance vocal range correlations................. 100

6.1.4 Other anthropometric correlations ............................. 101

6.1.5 Correlations between re-tested parameters................. 102

$7 \quad$ Discussion ....................................................... 103

Main findings..................................................... 103

7.2 Comparison with previous studies ................................ 105

7.2.1 Anthropometric indices ....................................... 105

7.2.2 Neck measurements ............................................ 106

7.2.3 CT space and laryngeal cartilages........................... 107

7.2.4 Speaking Fundamental Frequency ........................... 109

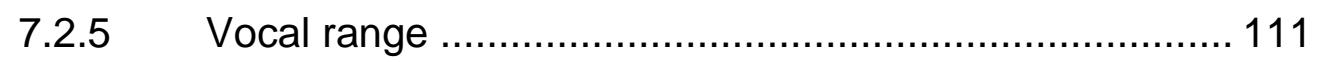

$8 \quad$ Practical Implications ........................................ 114

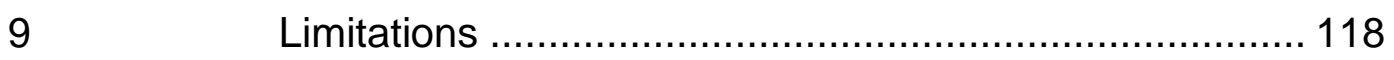

9.1 General limitations ........................................ 118

$9.2 \quad$ Measurement errors .............................................. 118

9.3 Measurement reliability ...................................... 120

$9.4 \quad$ Suggestions for improvements ............................. 121

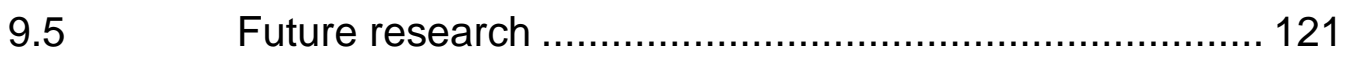

$10 \quad$ Conclusions ..................................................... 123 
Appendix A: Surgical procedures for pitch modification 152

Appendix B: Voice types ...

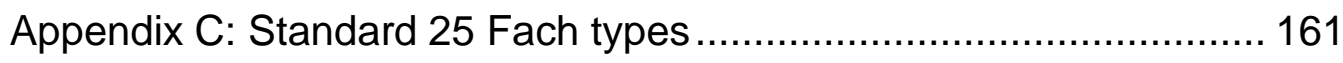

Appendix D: Cadaver studies on the cricothyroid joint ...................... 163

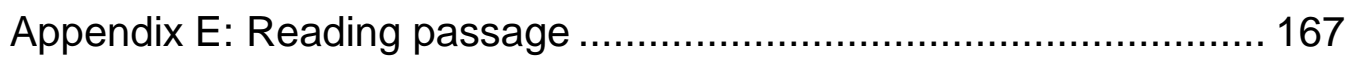

Appendix F: Ethical approval, information sheet and consent form .... 168

Appendix G: Summary of anthropometric data divided into voice types soprano and mezzo-soprano

Appendix H: Summary of acoustic data divided into voice types soprano and mezzo-soprano ........................................ 176

Appendix I: Data comparing age to SFF ...................................... 177

Appendix J: Conversion of note names to numbers .......................... 178

Appendix K: Repeated measurements raw data ............................... 179

Appendix L: Relationship between practice and performance vocal range

Appendix M: Correlations of all data.............................................. 182

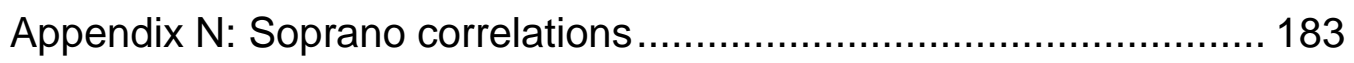

Appendix O: Mezzo-soprano correlations ........................................ 184

Appendix P: Data summary of CT space measurements $(\mathrm{mm})$ with vocal range in semitones, lowest and highest performance notes and Fach.

Appendix Q: Data comparing age to performance vocal range 186 


\section{List of Figures}

Figure 2.1: Sine wave showing compression and rarefaction ................... 2

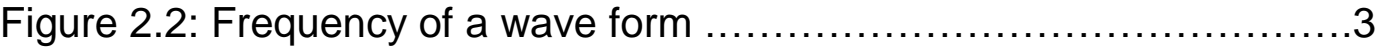

Figure 2.3: Phases of the vocal fold glottal cycle .................................. 6

Figure 2.4: Glottal cycle .............................................................. 7

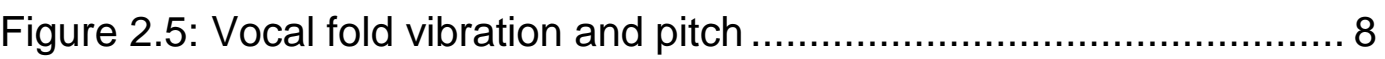

Figure 2.6: Cross section of vocal fold .............................................. 9

Figure 2.7: Narrowband sound spectrogram showing fundamental frequency (F0) and harmonics (H) ................................... 14

Figure 2.8: Average $F_{0}$ as a function of age for trained and untrained men and women .................................................................. 18

Figure 2.9: Schematic representation of CTA ...................................... 22

Figure 2.10: Anterior Commissure Laryngoplasty .................................. 23

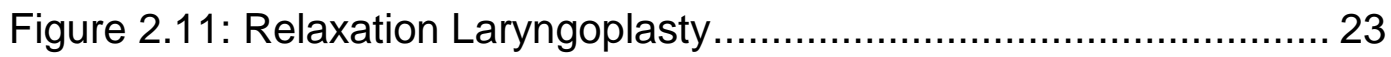

Figure 2.12: Principle resonating cavities; pharynx and oral cavities ....... 32

Figure 2.13: Larynx: Anterior view ..................................................... 36

Figure 2.14: Larynx: Left anterior oblique view .................................... 37

Figure 2.15: Arytenoid cartilage movement .......................................... 38

Figure 2.16: Arytenoid and corniculate cartilages ................................ 39

Figure 2.17: Schematic cross section of a vocal fold ............................. 44

Figure 2.18: Vocal folds adducted and abducted ............................... 45

Figure 2.19: Lateral and superior view of the laryngeal intrinsic muscles 46

Figure 2.20: Intrinsic Laryngeal Muscles.............................................. 48

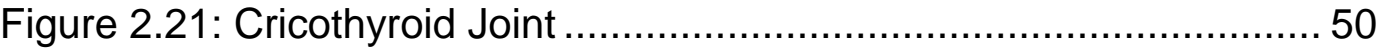

Figure 2.22: Rotation and translation of the cricothyroid joint .................. 54

Figure 2.23: Cricothyroid Space........................................................ 56

Figure 2.24: Correlation between the width of the CT space and the length of the vocal folds 59

Figure 2.25: Ultrasound image showing cartilaginous structures of the

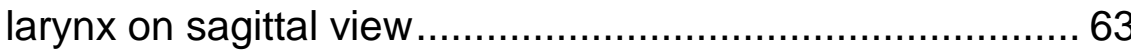

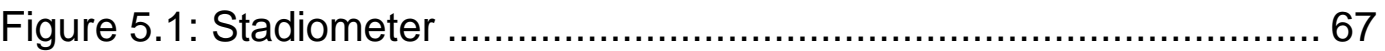

Figure 5.2: Scales showing readings obtained .................................... 67

Figure 5.3: Neck circumference measurement ..................................... 68 
Figure 5.4: Neutral head position using Frankfurt plane 69

Figure 5.5: Device used for anatomical positioning of head 69

Figure 5.6: Procedure for neck length measurement. ........................... 70

Figure 5.7: Sound spectrogram from a participants reading passage...... 72

Figure 5.8: Illustrates the examination procedure 74

Figure 5.9: Longitudinal sagittal ultrasound image of larynx with measurements

Figure 6.1: Box and whisker plot and tabular mean values of age for each voice type 79

Figure 6.2: Box and whisker plot and tabular mean values of height for each voice type 80

Figure 6.3: Box and whisker plot and tabular mean values of weigh for each voice type

Figure 6.4: Box and whisker plot and tabular mean values of BMI for each voice type

Figure 6.5: Box and whisker plot and tabular mean values of neck circumference for each voice type 81

Figure 6.6: Box and whisker plot and tabular mean values of neck length for each voice type

Figure 6.7: Box and whisker plot and tabular mean values of cricoid cartilage arch height for each voice type.... 82

Figure 6.8: Box and whisker plot and tabular mean values of cricothyroid space dimensions for each voice type

Figure 6.9: Cricothyroid space measurements divided into soprano and mezzo-soprano

Figure 6.10: Cricothyroid space measurements according to Fach 84

Figure 6.11: Box and whisker plot and tabular mean values of thyroid cartilage height for each voice type

Figure 6.12: Box and whisker plot and tabular mean values of combined length of laryngeal cartilages for each voice type

Figure 6.13: Box and whisker plot and tabular mean values of speaking fundamental frequency for each voice type

Figure 6.14: Box and whisker plot and tabular mean values of lowest performance note for each voice type 
Figure 6.15: Relationship of lowest performance note to voice type 88

Figure 6.16: Box and whisker plot and tabular mean values of highest performance note for each voice type

Figure 6.17: Relationship of highest performance note to voice type....... 89

Figure 6.18: Box and whisker plot and tabular mean values of performance vocal range for each voice type

Figure 6.19: Box and whisker plot and tabular mean values of lowest practice note for each voice type 90

Figure 6.20: Box and whisker plot and tabular mean values of highest performance note for each voice type

Figure 6.21: Box and whisker plot and tabular mean values of practice vocal range for each voice type

Figure 6.22: Relationship between cricothyroid space and speaking fundamental frequency

Figure 6.23: Relationship of the cricothyroid space to the lowest performance note. 95

Figure 6.24: Relationship of the cricothyroid space to the lowest performance note in sopranos 96

Figure 6.25: Cricothyroid space showing lowest performance notes of sopranos and mezzo-sopranos

Figure 6.26: Relationship of anterior cricothyroid space with highest performance note. 98

Figure 6.27: Cricothyroid space showing highest performance notes of sopranos and mezzo-sopranos 98

Figure 6.28: Relationship of anterior cricothyroid space with performance vocal range

Figure 6.29: Relationship between first and second measurements on selected parameters 102

Figure A12.1: Drawing of a larynx showing movements of the thyroid cartilage secondary to movements at the CTJ 163

Figure A12.2: CT joints according to Maue and Dickson 164

Figure A12.3: Different locations of the effective rotational axis of the CTJ 


\section{List of Tables}

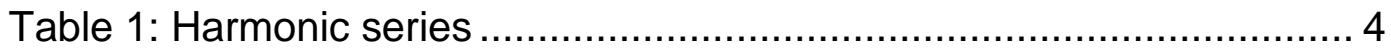

Table 2: Variables influencing SFF .............................................. 15

Table 3: Dimensions of the adult larynx in cadavers.............................. 40

Table 4: Comparison of cricoid and thyroid cartilage dimensions in different ethnic groups............................................................ 42

Table 5: Anthropometric indices, laryngeal cartilage dimensions, and the cricothyroid space data for the study sample ………….............. 77

Table 6: Acoustic data of the study sample ......................................... 78

Table 7: Comparison of speaking fundamental frequency in participants younger or older than 40 years of age

Table 8: Re-tested parameters showing differences in the mean, standard deviation and standard error of the mean for the selected measurements.

Table 9: Summary of selected parameters according to voice type

expressed as means and standard deviations 104

Table 10: Comparison of neck dimensions 106

Table 11: Comparison of female thyroid and cricoid cartilage measurements and cricothyroid space with previous studies . 108

Table 12: Comparison of average SFF values in voice types 109

Table 13: Comparison of average performance vocal ranges between sopranos and mezzo-sopranos

Table 14: Comparison of average performance vocal ranges between Fach

Table 15: Summary of pitch raising mechanisms and their effect on the CT space

Table A16: Relaxation laryngoplasty: surgical procedure 156 


\section{Glossary}

2-sample t-test

Abduction

Adduction

Amplitude

Antagonist muscle

Anterior

Anteroposterior

Arthritis

Atrophy

Average

Axial plane

Body mass index

(BMI)
A hypothesis test for two population means to determine whether they are significantly different A body movement away from midline. In the larynx, abduction is when the vocal folds separate to open the glottis for breathing

A body movement toward the midline. In the larynx, adduction is when the vocal folds come together to close the glottis

The height of a sound wave, which give the intensity of sound. Measured in 'bels', named after Alexander Graham Bell who invented the telephone, often the decibel scale is used which allows the enormous range of intensities to be broken down into manageable portions. $1 \mathrm{db}$ is $1 / 10^{\text {th }}$ of a bel

A muscle that opposes the action of another. In the vocal folds the TA and CT muscles are antagonistic Front side of the body, also known as ventral From front to back Inflammation of joints Wasting away of tissues (mostly commonly muscle). This typically occurs as a result of disuse Also known as the mean: is the sum of all the observations divided by the number of observations Also known as the horizontal plane. Divides the body into superior and inferior parts, roughly perpendicular to the spine

A ratio between weight and height that is commonly used to classify obesity in adults. The equation is body weight $(\mathrm{kg}) /$ height $^{2}(\mathrm{~m})^{2}$ which gives $\mathrm{BMI}$ in $\mathrm{kg} / \mathrm{m}^{2}$ 
Boxplot

Caudal

Closed quotient

Correlation

Cranial

Cricoid cartilage

Cricothyroid membrane

(Cricothyroid ligament) Connects the front parts of the thyroid and cricoid cartilages

Cricothyroid muscle A paired intrinsic laryngeal muscle that is used primarily to control pitch

Damping

Distal

Dizygotic twins

Electromyography

External muscles

Fach

Fast Fourier

transform (FFT)

Formant

Frankfurt horizontal

plane

A graphical summary of the distribution of a sample that shows its shape, central tendency, and variability

Rear side of the body, also known as the posterior Percentage of glottal cycle where the glottis is adducted or closed

A measure of linear association between two variables

Above or near the head, also known as superior Ring shaped cartilage connected to the trachea Shortening of the vibrating length of the vocal folds Away from the trunk

Also known as fraternal twins: develop from two eggs, each fertilized by separate sperm cells

An electrical recording of muscle activity

Muscles on the external (outside) of an anatomical structure (as opposed to intrinsic/inside muscles) Speciality or category (German)

A Fourier transform converts time to frequency. It shows the sounds spectral content divided into frequency bands. The FFT is a common algorithm for Fourier transforms and rapidly computes such transformations

A point of concentrated energy within the vocal sound wave

A plane that passes through the orbitales and the porions to make a horizontal plane. The head is 
positioned parallel to the ground. Established in 1884 in Frankfurt, Germany, by the World Congress of Anthropology and is decreed as the principal standard anatomical position for representation of the human skull

Frequency

The number of vibrations or cycles per unit of time, usually expressed in Hertz $(\mathrm{Hz})$. In voice it refers to the number of times the vocal folds open and close in a second

\section{Fundamental}

frequency $\left(\mathrm{F}_{0}\right)$

The lowest frequency in a sound wave

Glottal cycle One glottal cycle is an open and close of the glottis

Glottal period

Glottis Time interval between closing instances of the glottis

The opening between the vocal folds when they are separated (abducted)

Harmonics

Hertz (Hz)

Horizontal

Hypoechoic

Inferior

Inter-rater

repeatability

Intraluminal
An integral multiple of the fundamental frequency. Overtones of a complex sound

The number of cycles of a vibrating object in one second, a unit of frequency named after the 19th C. German Physicist Heinrich Hertz

A plane passing through the standing body parallel to the floor dividing the body into superior and inferior parts

A tissue or structure that reflects relatively few ultrasound waves, gives off few echoes.

Below, towards the feet

A method of measuring reliability which determines the extent to which two or more raters obtain the same result when using the same instrument to measure a concept

Within the lumen of a tubular structure or organ, between or among tubes 
Intra-rater

repeatability

A measure of reliability assessment in which the same assessment is completed by the same rater on two or more occasions

Intrinsic muscles Muscles on the internal (inside) of an anatomical structure, as opposed to the extrinsic (outside) muscles

Isometric tension When two antagonistic muscles contract at the same time causing an increase of tension. When the CT and TA reach an isometric point, vocal fold tension is increased, but no change in vocal fold length occurs

Lamina propria

Laryngeal

Larynx

Lateral

Limitations

Lower whisker

Mean

Medial

Median

Median plane
Layers in motion

Relating to the larynx

The structure of muscle and cartilage at the upper end of the trachea, containing the vocal folds. It serves as the human organ of vocal sound. Also known as the voice box

To the side of the body

Those elements over which the researcher has no control

Extends to the minimum data point within 1.5 box heights from the bottom of the box in a boxplot graph

Also known as the average: is the sum of all the observations divided by the number of observations To the middle of the body

The middle of the range of data: half the observations are less than or equal to it and half the observations are greater than or equal to it A vertical plane dividing the body into left and right halves. Also known as the mid-sagittal plane 
Mid-sagittal plane

Monozygotic twins

Oedema

Orbitale

Ossification

Outlier

Paired t-test

Palate

Pearson product

moment

correlation (r)

Pedagogy

Period

Periodic

Pharynx

Pitch

Porion

Posterior

Posteroanterior
A vertical plane dividing the body into left and right halves. Also known as the median plane

Also known as identical twins. They develop from one zygote that splits and forms two embryos that have completely identical genotypes

Excessive accumulation of fluid in tissues

Lowest point of the lower margin of the orbit (eye socket)

Turn to bone

Observation that is beyond the upper or lower whisker in a boxplot

A hypothesis test for the mean difference between paired observations that are related or dependent The roof of the mouth (buccal cavity) which consists of the alveolar ridge and the hard and soft palate

Assesses whether two continuous variables are linearly related. The measure of association varies from -1 to +1 , with 0 indicating non relationship and 1 indicating perfect relationship

The science and art of teaching

The time interval between repeating events Has a regular repetition rate, and thus a pitch, that can be expressed as a frequency in hertz $(\mathrm{Hz})$

The throat, specifically the vocal tract from the back of the nose down to the top of the larynx

The psychoacoustic counterpart of fundamental frequency (F0)

Most lateral point on the roof of the external bony ear holes

Rear side of the body, also known as caudal From back to front 
Prone

Proximal

Rarefaction

Real-time

Registers

Repeatability

Reproducibility

Resonance

Sagittal plane

Senescence

Singers formant

Sinuses

Sinusoidal motion

Soft palate

\section{Speaking}

fundamental

frequency

Spectrogram
With the front or ventral surface lying downward (lying face down)

Near the trunk

A decrease in density

A display that appears virtually simultaneously with the sound that generates it

Perceptually distinct regions of vocal quality as pitch or loudness is changed

represents the variation that occurs when the same appraiser measures the same part with the same device

represents the variation that occurs when different appraisers measure the same part with the same device

Acoustical amplification and reinforcement of sound vibrations

A vertical plane passing through the standing body from front to back

The condition or process of deterioration with age

A strong area of energy centred around 2800$3500 \mathrm{~Hz}$ and generally found among a clustering of the third, fourth and fifth formants. Sometimes called the 'ring' in the voice paranasal cavity The projection of circular motion (in a plane) at constant speed onto one axis in the plane Velum: the soft posterior portion of the roof of the mouth

The average rate at which the vocal folds vibrate in connected speech

A visual representation of sound. A short segment of sound represented visually which shows three 
dimensions: frequency (in Hertz), intensity (colour spectrum), and time (ms)

Staccato

Stadiometer

Sub-glottal

Superior

Supine

Supra-glottal

Tessitura

Testosterone

Thorax

Thyroarytenoid

muscle

Thyroid cartilage

Timbre

Trachea

Transverse

Upper whisker
A note separated from successive notes by being quickly released

An instrument used to measure height

Below the glottis

Above or near the head, also known as cranial

With the back or dorsal surface downward (lying face up)

Above the glottis

Area within a singer's vocal range that is produced with minimal strain

The hormone responsible for development of male sexual characteristics, which includes major growth in the larynx

The chest cavity, containing the heart, lungs, part of the trachea and the oesophagus

A paired intrinsic laryngeal muscle that comprises the bulk of the vocal fold

A "V" shaped cartilage in the neck. Also known as the "Adams apple" in men

The characteristic quality of sound determined by the harmonics, which distinguishes one voice or instrument from another

The windpipe, main tubular system by which air passes to and from the lungs

A horizontal plane passing through the standing body parallel to the ground, i.e. in a crosswise direction. This plane divides the body into upper (superior) and lower (inferior) regions

Extends to the maximum data point within 1.5 box heights from the top of the box in a boxplot graph 
Uvula

Velum

Ventral

Vertical

Vibrato

Vocal folds

Vocal ligament

Vocalis muscle
Pendular muscle at the posterior of the velum or soft palate, which hangs like a sac

The soft palate; the soft posterior portion of the roof of the mouth

Front side of the body, also known as anterior Upright

A pulse in the voice produced by alternating perceptible variation and pitch

Part of the larynx which is comprised of the vocalis muscle, the vocal processes of the arytenoid cartilage and the vocal ligament

Elastic tissue membrane extending from the thyroid cartilage in front to the vocal process of the arytenoid cartilage behind

Also known as the internal thyroarytenoid, and is adhered to the vocal ligament 


\section{List of Abbreviations}

BMI Body mass index

CAT Computed axial tomography

cm Centimetre

CT Cricothyroid

CTA Cricothyroid approximation

CTJ Cricothyroid joint

dB Decibel

EMG Electromyography

Fo Fundamental frequency

FFT Fast Fourier transform

H Harmonic

Hz Hertz

mm Millimetre

SFF Speaking fundamental frequency

TA Thyroarytenoid 


\section{Introduction}

In a recent summer course for singing, an observation was made on the length of my thyroid and cricoid cartilages, and my small cricothyroid (CT) space. The professor, when questioned, was uncertain as to its significance. This piqued my curiosity as to what influence the CT space and length of the thyroid and cricoid cartilages would have on a singer's voice.

The CT space is the interval between the anterior inferior border of the thyroid cartilage and the anterior superior border of the cricoid cartilage. An article by Ingo Titze (1998a), Professor of Speech Science and Voice at the University of lowa, and Executive Director of the Wilbur James Gould Voice Research Center for the Performing Arts, entitled "Five ingredients of a physiologically gifted voice" suggested that the CT space was one of the most important anatomical dimensions of the larynx in determining vocal range. He previously noted that the vocal ligament may make a difference to the extent of the pitch range (Titze, 1996). This hypothesis was both logical and intriguing, and I therefore decided to investigate it further.

The sound of the voice is created in the larynx by the vibration of the vocal folds. The cricothyroid space in the larynx closes and opens to modulate vocal pitch. The ability to be able to produce voice over such a wide pitch range is unique to humans. Could the natural dimensions of the cricothyroid space be a key factor in determining a singer's vocal range? This thesis explores this relationship, and its association with anthropometric indices, fundamental frequency and voice classification. 


\section{Literature review}

\subsection{Nature of Sound}

Sound is an oscillating pressure wave that is transmitted through a medium (gas, liquid or solid) and composed of frequencies detectable by the hearing apparatus. This section focuses on sound transmitted by air molecules to the human ear. The sound wave travels through the air by the pressurisation and immediate depressurisation of air molecules, thus causing them to be alternately compressed and rarefacted. When this disturbance of air molecules reaches the ear, it results in a vibration in the tympanic membrane (ear drum), interpreted as sound by the brain (Michael, 2012).

A sound wave can be depicted graphically as a waveform, a visual representation of the compressions and rarefactions that occur in a given time (Figure 2.1). This waveform allows key features to be easily identified which aid with interpretation of a sound wave. A period is one cycle of vibration (compression and rarefaction). The horizontal line of the waveform is the point of equilibrium, whilst anything above the line is positive pressure (compression) of the air molecules and anything below the line is negative pressure (rarefaction).

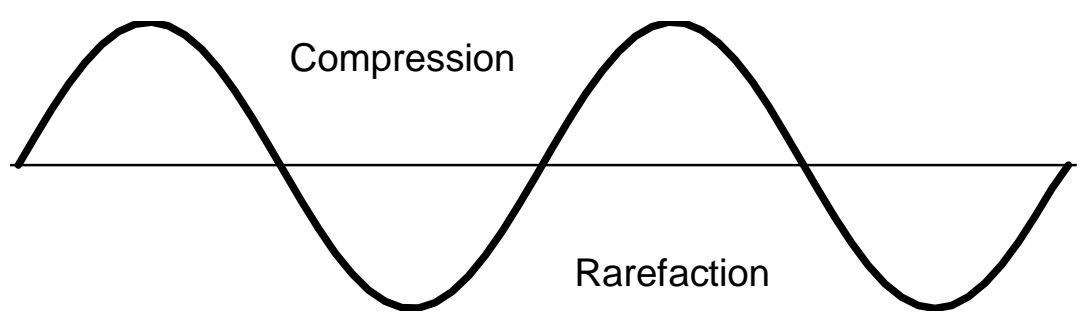

Figure 2.1: Sine wave showing compression and rarefaction

A sine wave represents a vibration at one single frequency and amplitude, with equal compression and rarefaction phases. A waveform like this does not exist in nature and is not representative of the human voice. Sounds in the real world are the consequence of complex vibrations, made up of 
several sine waves occurring simultaneously with differing frequencies, amplitudes and phases (Michael, 2012).

\subsubsection{A musical sound wave}

Musical and non-musical sounds are differentiated by the regularity of the vibration. A musically pitched sound has vibrations which are strong and regular (periodic). When a regular vibration is heard then a specific frequency is detected and a pitch is perceived. Conversely, a musically non-pitched sound is a mixture of different frequencies with no strong regularity such that a musical tone or specific pitch cannot be detected (How Music Works, 2012).

\subsubsection{Characteristics of a regular sound wave}

There are four main properties of a regular sound wave which affect the way it is heard. These are frequency (or pitch), amplitude (loudness), the tone quality (timbre), and the duration.

- Frequency (pitch)

Water and ice describe the same thing but from a different viewpoint and something similar may be said regarding frequency and pitch (a musical note). They are closely and directly related but not equivalent. Frequency is objective whilst pitch is subjective. Frequency is measured in cycles per second or Hertz $(\mathrm{Hz})$ and is the speed of vibration. One Hertz is one waveform occurring in one second. There is a reciprocal relationship between frequency and period, frequency being inversely proportional to the wavelength (length of a period). Figure 2.2 illustrates this relationship.

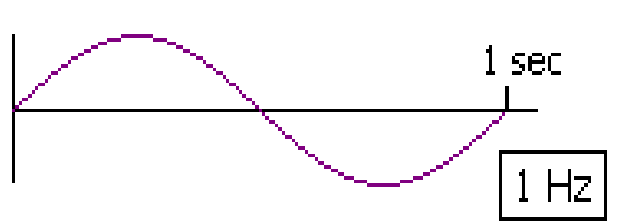

Low frequency

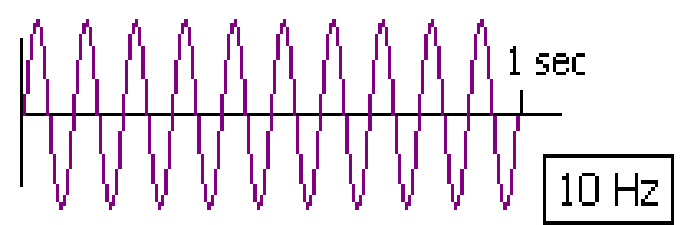

Higher frequency

Figure 2.2: Frequency of a wave form (How Music Works, 2012; used with permisssion) 
Pitch is the subjective interpretation of frequency as perceived by the ear (Hass, 2003; Shewell, 2009). This equates to how low or high the sound appears to the hearer. The frequency of vibration gives us the pitch of the sound: the higher the frequency the higher the pitch.

Each musical note has its own distinctive frequency. The lowest or fundamental frequency is also known as the first harmonic (H1). Associated harmonics are whole number multiples of the fundamental frequency. This relationship between the fundamental and its harmonics is referred to as the harmonic series (McCoy, 2004) (Table 1).

Table 1: Harmonic series

\begin{tabular}{|c|c|}
\hline Harmonic $(\mathrm{H})$ & Musical Interval \\
\hline $\mathrm{H} 1-\mathrm{H} 2$ & Octave \\
\hline $\mathrm{H} 2-\mathrm{H} 3$ & Perfect fifth \\
\hline H3- H4 & Perfect fourth \\
\hline H4-H5 & Major third \\
\hline H5-H6 & Minor third \\
\hline H6-H7 & Minor third \\
\hline
\end{tabular}

- Amplitude (loudness)

Amplitude, measured in decibels $(\mathrm{dB})$, is the strength of the vibration, which is proportional to the loudness of the sound. The greater the amplitude the louder the sound, although this is not a direct correlation since other factors can also affect the perception of loudness. 
- Timbre

Two notes of the same pitch and loudness from the same or different instruments, may sound quite different due to the differences in tone quality (timbre). The sound waves associated with each note are complex, consisting of many waves occurring simultaneously. This mixture of different but related frequencies (harmonics), which occur with varying prominence, plays an important part in giving each instrument its distinctive tone or timbre.

- Duration

The duration of a sound wave is a measure of the time for which it remains detectable. Musical sounds have to be of sufficient duration to establish a periodicity for the brain to interpret it as pitch.

\subsubsection{How the larynx makes a sound}

The human larynx creates sound waves by vibrating the vocal folds (Deguchi et al., 2011). The sound waves then travel through the vocal tract and out into the air (Michael, 2012).

During respiration when the vocal folds are abducted (separated) air moves freely in and out of the body. During singing or speaking the vocal folds adduct (close) obstructing this air flow. This causes the vocal folds to vibrate in a cycle of closing and opening, alternating the trapping and releasing of air which creates the compressions and rarefactions that cause sound. Each trap/release cycle of vibration can be referred to as a glottal cycle, which may be divided into four phases: A closed phase, B opening phase, $C$ open phase, and D closing phase (Figure 2.3). These phases are not the result of multiple laryngeal muscle contractions as is commonly believed. The intrinsic muscles of the larynx do play their part in stabilising the vocal folds, but the actual vibration of the folds is due to the change in air pressure that occurs (Bernoulli principle) as explained below (Wolfe et al., 2010). 


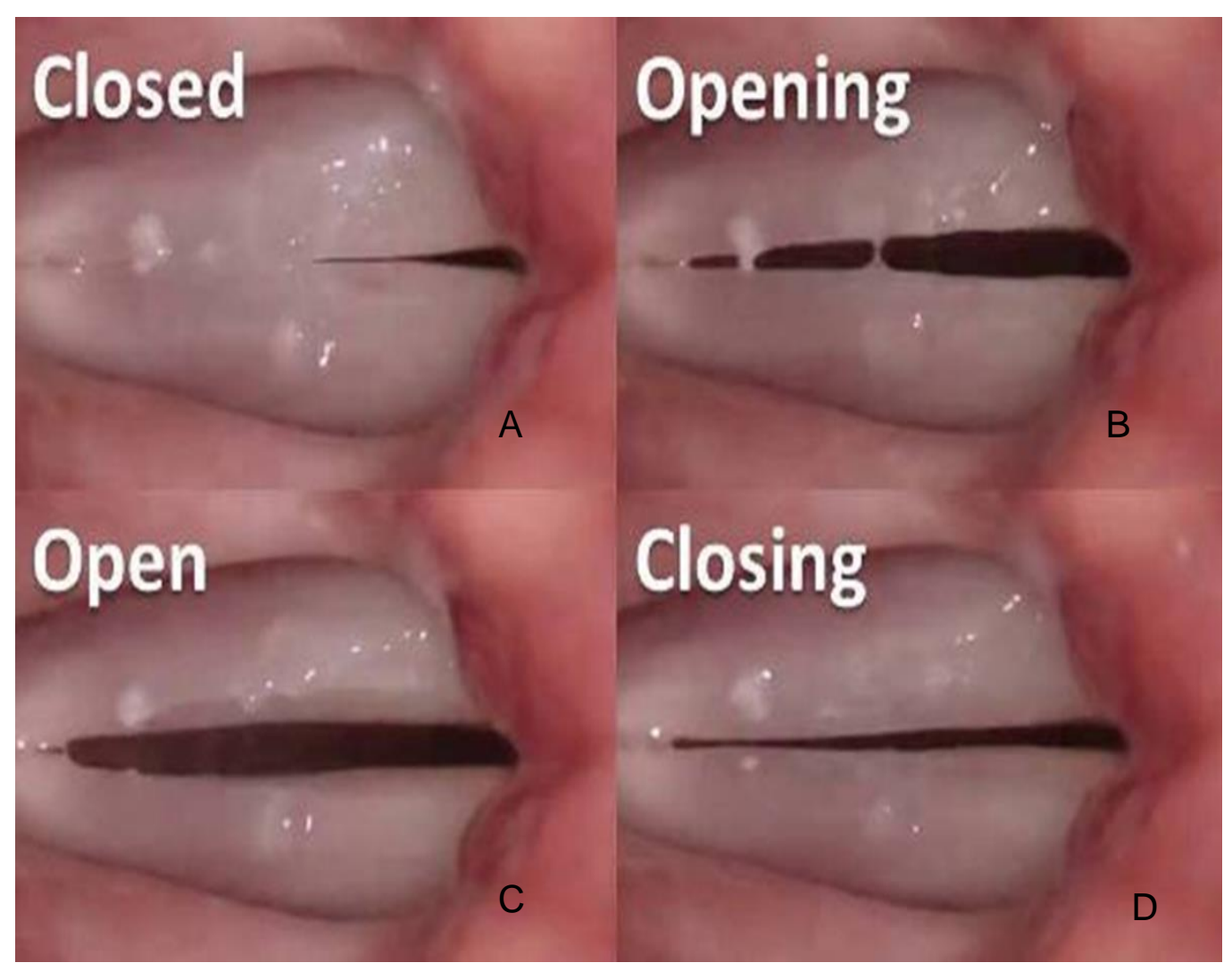

Figure 2.3: Phases of the vocal fold glottal cycle. A closed phase: B opening phase: $C$ open phase and D closing phase (Little, 2012)

Figure 2.4 is a schematic diagram of the vocal folds in cross section showing the phases of the glottal cycle. In the closed phase (1) the airstream is momentarily stopped when the vocal folds are adducted causing the subglottal pressure to build up below the vocal folds. Opening phase 2, 3: When the subglottal pressure below the vocal folds is high enough, the folds are forced apart (opening phase [2 and 3]). In the open phase (4 and 5) high speed air flows through the vocal folds lowering the subglottal pressure (beginning of the sound wave). This is followed by the closing phase (6 to 9 ) when the drop in subglottal pressure sucks the vocal folds back together with the lower part of the folds closing first, followed by the upper part. Finally the vocal folds are once again closed (10) cutting off the air column. The vibratory cycle then repeats (Jiang et al., 2000; Reeve, 2005; Chapman, 2006). 

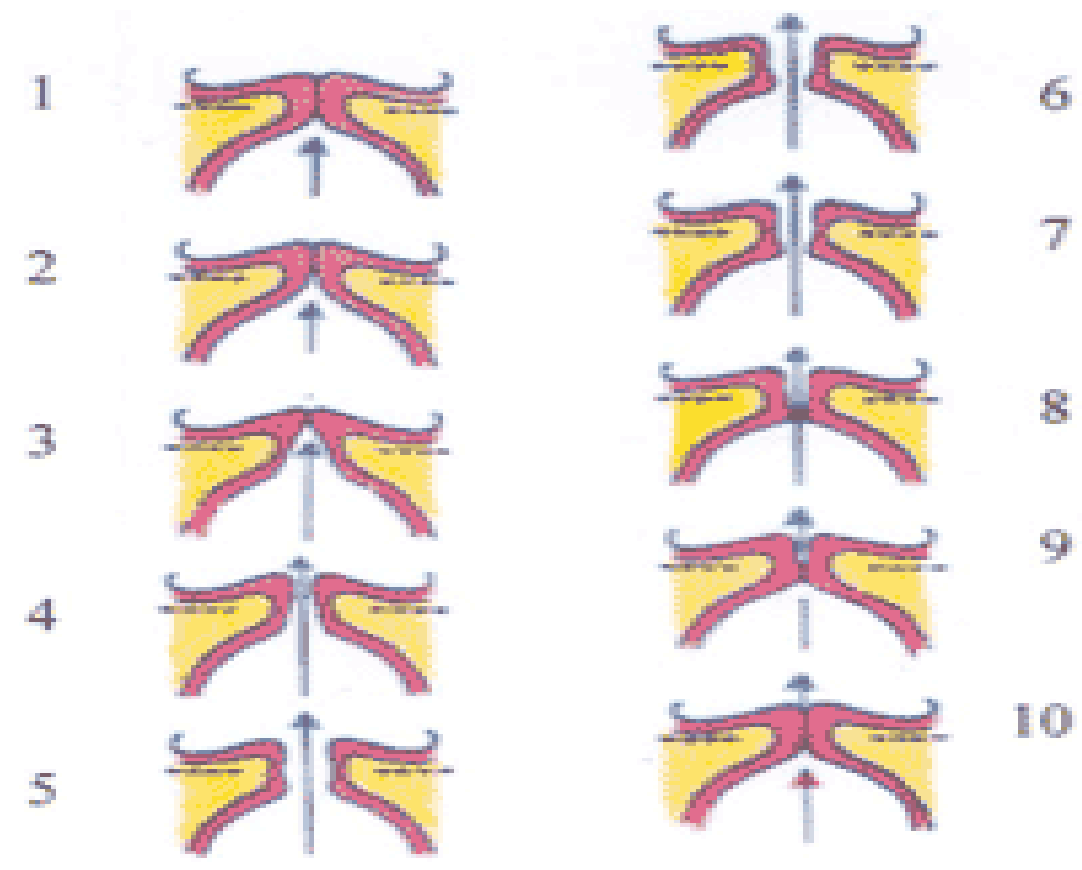

Figure 2.4: Glottal cycle. 1 Closed phase: 2 and 3 opening phase: 4 and 5 open phase: $6,7,8$ and 9 closing phase: 10 closed phase (Washington Voice Consortium, 2013)

- Vocal fold frequency

The primary determinant of vocal fold vibration frequency and therefore fundamental frequency (pitch) is tension on the vocal folds. The role of the cricothyroid and thyroarytenoid muscles in lengthening and shortening the vocal folds respectively, is discussed in Chapter 2.5.4. Lengthening the vocal folds increases their tension and elasticity but their edges become thinner so only the superficial parts of the vocal fold vibrate. This increases the frequency of vibration and therefore the pitch. Conversely, shortening the vocal folds reduces their tension and elasticity, causing deeper parts of the vocal fold to vibrate. This reduces the frequency of vibration and thus lowers the pitch (National Center for Voice and Speech, 2013a).

In voiced sounds, the lowest fundamental frequency is usually dominant and the one perceived as pitch. For example, the fundamental frequency $440 \mathrm{~Hz}$ is equivalent to the pitch of the musical note A4 (the A above middle $C$ ). When this note is sung it means that the vocal folds are opening and closing 440 times in one second. Doubling the frequency of 
vibration results in a note perceived as an octave higher. Figure 2.5 shows how fast the vocal folds are vibrating at certain pitches.

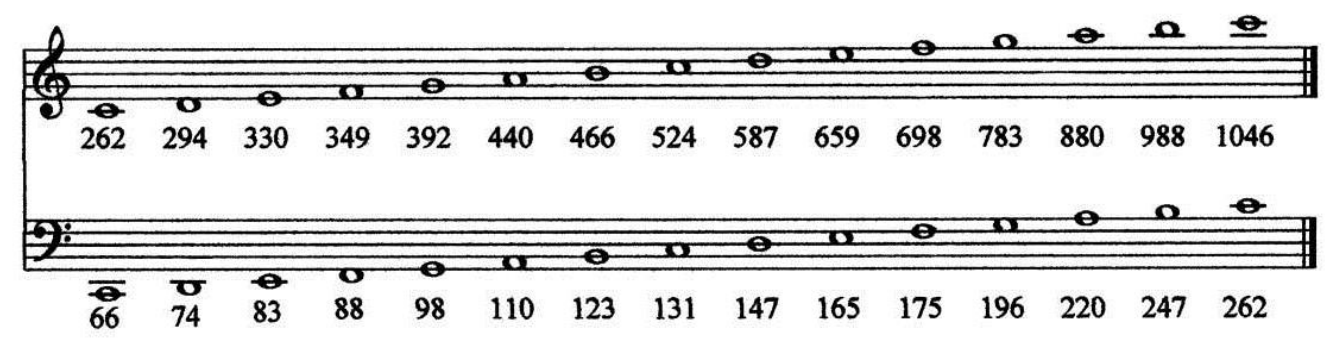

Figure 2.5: Vocal fold vibration and pitch. The frequency of vocal fold vibration $(\mathrm{Hz})$ is shown beneath each note (Michael, 2012; used with permission)

- Vocal fold structure and composition

The structure and composition of the vocal folds regulates vocal fold tension, and this is also important in determining $F_{0}$ at which the vocal folds will vibrate (Titze, 1981; Colton, 1988; Jiang, et al., 2000). The vocal folds are multilayered structures comprising muscle and connective tissue with a mucosal covering (discussed in section 2.5.3).

The tension developed within the vocal folds is determined by the elastic and collagen fibres within it and covering its surface. Collagen fibres provide strength and stiffness, whereas elastic fibres provide elasticity (Titze, 1981). Thus, the greater the proportion of collagen fibres in a vocal fold layer, the more resistant to vibration it will be. The thin outer layer (cover) is made up of pliable, loose material that cannot hold much tension, but can stretch. This allows free vibration of the vocal cover over the body, resulting in the formation of a mucosal wave as air is passed through the glottis as a release of building subglottic pressure (Figure 2.6a). 

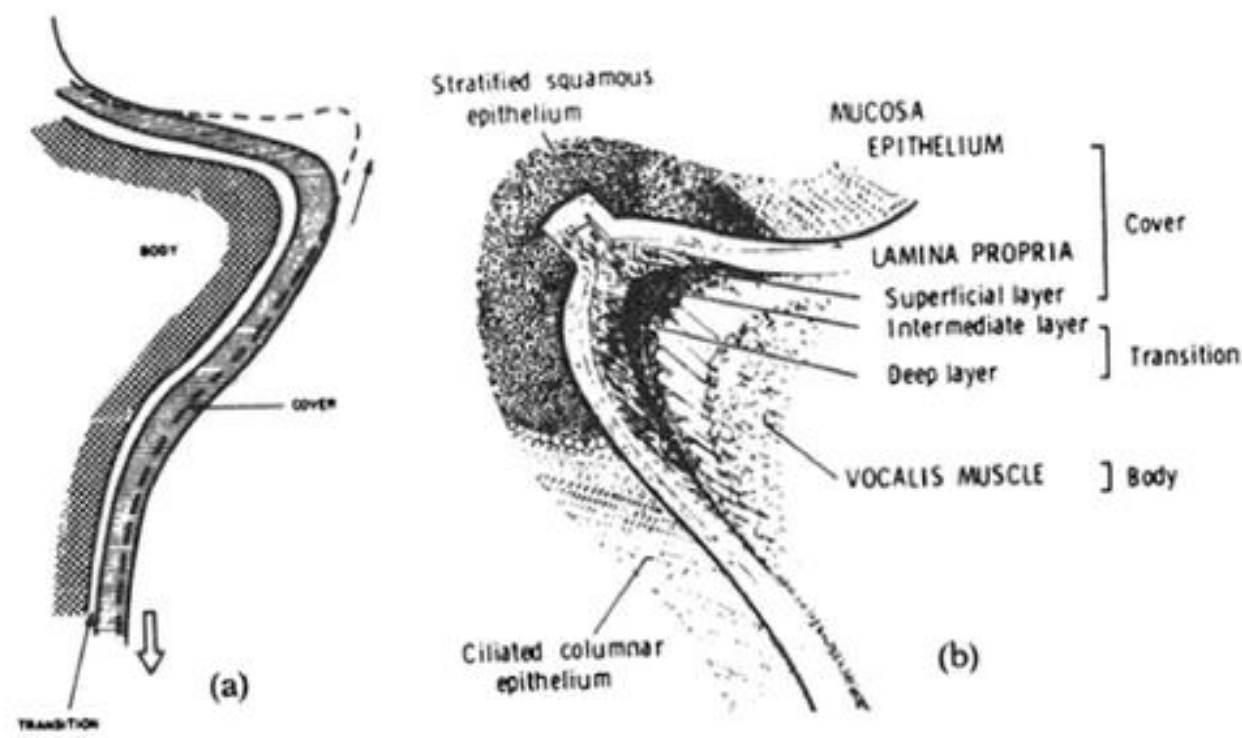

Figure 2.6(a): Schematic cross section of vocal fold showing cover, transition and body. The dashed line and upper arrow show the kind of sliding motion that is postulated to occur between cover and body. (b) A frontal section of a human vocal fold at the mid-point of the membranous portion, schematically presented (Miller, 1986b)

The transition layer of the vocal folds (also known as the vocal ligament) consists of the intermediate and deep layers of the lamina propria (Figure 2.6b). The intermediate layer of the lamina propria consists mainly of elastin fibres running parallel to the long axis of the vocal fold (Hirano et al., 1983; Sataloff, 1997; Chapman, 2006). Whereas, the deep layer of the lamina propria consists primarily of densely packed collagen fibres (Hirano, et al., 1983; Sataloff, 1997; Titze \& Hunter, 2004). Functionally, this layer limits vocal fold elongation, helps position the vocal folds when the arytenoid cartilage moves, and provides adhesion between the layers. The vocal ligament also gives the vocal folds support, particularly for highpitched sounds (Titze, 1996). The vocalis muscle (one of the intrinsic laryngeal muscles) makes up the body of the vocal fold.

- Mechanisms of vocal fold vibration

The vocal folds can vibrate in four different ways, determined by combinations of their length, mass and tension combined with the amount of time glottis is open or closed (Roubeau et al., 2004; Henrich, 2006). 
The first way is called, 'vocal fry' or 'creak' which is when the tension of the vocal folds is so low that the vibration is aperiodic. It sounds low but has no clear pitch (Hollien \& Michel, 1968). The second way is often referred to as thick fold vibration, and is used to produce low and medium pitches with virtually all of the mass and length of the vocal fold vibrating (Behnke, 1900). The frequency is regulated by muscular tension (Hirano et al., 2009). The glottis opens for a relatively short fraction of a vibration period (Henrich et al., 2005). Thin fold vibration, the third way, is used to produce medium and high pitches for women and high pitches for men. A reduced fraction of the vocal fold mass vibrates, amounting to about two thirds its length and less of their breadth. The glottis is open for a longer fraction of the vibration period (Henrich, et al., 2005). The fourth way is a phenomenon known as damping. Damping is used to describe the production of the highest range of pitches, called 'whistle' or 'flageolet. Damping occurs when there is a "reduction in the effective length of vibration of the vocal folds" (Titze \& Hunter, 2004: p. 6). Only the anterior part of the vocal folds vibrate as the posterior region of the vocal folds are approximated and do not vibrate (Miller, 2000; Titze \& Hunter, 2004).

Thick and thin fold vibrations are usually used in speech and singing. Some people use 'vocal fry' or 'creak' in speech, especially at the end of sentences, and coloratura sopranos use whistle or flageolet in their highest range. Men, with their lower vocal range, typically use thick fold for nearly all speech and most singing. When singing in 'falsetto' they use thin folds. For women singers, the situation depends on the vocal range. Thick folds are used for 'chest' voice and thin folds for 'head' voice. There is usually a pitch and intensity range over which singers can use either thick or thin fold, and trained singers are good at disguising the transition (Roubeau, et al., 2004). Altos often use both thin and thick folds although sopranos usually sing in thin folds and extend their range downwards to avoid the 'break' in the voice. High sopranos often have a considerable overlap region between thin fold and flageolet. 
- Amplitude of the vocal folds

In singing and speaking, the strength of vibration of the vocal folds is directly related to the strength of the air stream traversing the glottis each time it opens during a glottal cycle (cycle of vibration). This in turn is dependent on two interrelated factors: the length of time the vocal folds are closed and how much of the vocal folds are opposed during the glottal cycle. The role of the adductor muscles, (thyroarytenoid, lateral cricoarytenoid and interarytenoid), in bringing the vocal folds together is discussed in section 2.5.4.

In the glottal cycle when the vocal folds are closed together for a longer time a greater subglottal (underneath) pressure builds up and subsequently more vocal fold muscle is engaged to keep them adducted. A higher subglottal pressure is then needed to separate the folds which are more forcefully moved apart as the air explodes through the glottis. Consequently, more air is displaced creating a sound wave with a greater amplitude and hence a louder sound. Due to the Bernoulli Effect (Reeve, 2005), the open phase is now much shorter because the air passing through the vocal folds is moving faster and therefore at lower pressure so the vocal folds close faster. In soft phonation, less of the vocal folds are engaged in closing them and the closed phase is shorter as the subglottal air pressure does not build up to such a high level. The expulsion of air through the glottis is weaker causing a smaller amplitude, thus a quieter sound (Michael, 2012).

- Vocal timbre

The tone quality in the voice is affected by the resonating chambers in the pharynx, mouth, nasal cavity and paranasal sinuses. The exact size and space of these vary between individuals giving each human voice its individual characteristics (Sataloff, 1997; Tortora \& Derrickson, 2006). 
- Vocal duration

In singing, the length of time a note is sung has to be sufficient to establish periodicity so the brain can interpret it as pitch. Even a staccato note lasting a few vibratory cycles can fulfil this requirement (McCoy, 2004).

- Wavelength

Wavelength is an important property of sound for singers because of the way different wavelengths propagate. This phenomenon affects singers when they are evaluating the pitch and quality of their own voices. A sound wave with a long wave length (low frequency) bends around obstacles and travels easily from the mouth to the ear making it easy for each singer to hear their sound. In contrast, a sound wave with a short wavelength (high frequency) travels in a straight line from the mouth making it harder for the singer to hear themselves (McCoy, 2004). 


\subsubsection{Sound Spectrogram}

A real time sound spectrogram using Fast Fourier transform (FFT) is a visual representation of a short sample of sound. The display plots a linear frequency which some singers may find difficult to interpret. Spectrograms can either be narrowband or wideband depending on whether frequency is divided into narrow or broad bands, respectively. As band width widens, frequency displays becomes less detailed. Narrow band spectrograms allow clear visualisation of harmonics and the wiggle or sinusoidal movement of them depicts vibrato, if present. With wideband spectrograms individual harmonics are no longer seen but spectral peaks (formants) are shown with great clarity. An interruption in the harmonic bands on a spectrogram is due to consonants in the vocal line. Therefore, the continuousness of signal represents legato (McCoy, 2004).

Three types of information are provided by a sound spectrogram: frequency (pitch), time, and amplitude (loudness).

Frequency (pitch) is shown on the vertical or $Y$ axis of the spectrogram and ranges from $0-5000 \mathrm{~Hz}$. Vibration at individual frequencies is displayed as horizontal lines, with the fundamental frequency (F0) being the lowest band. The bands above it represent the harmonics, which follow the pattern of the harmonic series (as explained in 1.4) and formants. The midpoint is $2500 \mathrm{~Hz}$ (E8) and therefore eight octaves are displayed below this line, whilst one octave is represented above the line (Miller, 2008).

The sound spectrogram reads like music, from left to right, with time shown on the horizontal or $\mathrm{X}$ axis. Between 4 and 20 seconds are visible in a single panel (Miller, 2008).

A sound heard as a single pitch can be analysed to show individual harmonics, each with their own intensity. The third dimension of a sound spectrogram is the intensity, or amplitude (dB's). This intensity (loudness) is represented by a colour change, with red being the loudest through yellow and green down to blue as the quietest (Miller, 2008). 


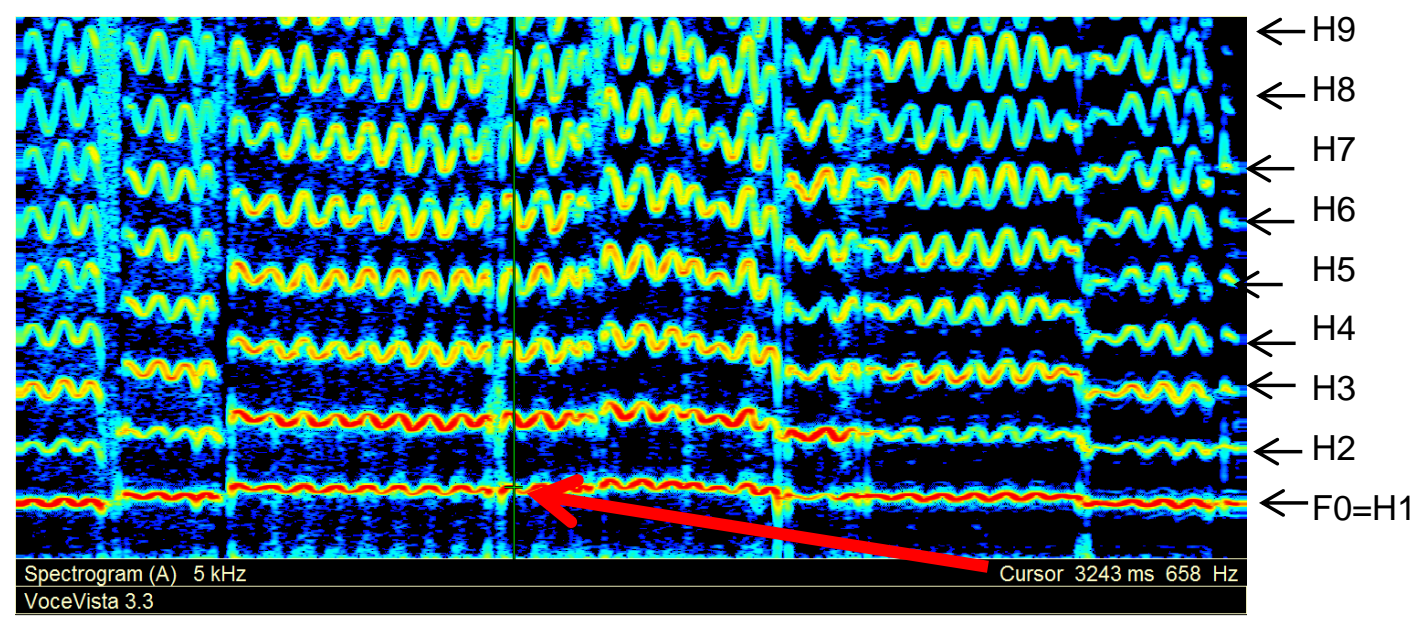

Figure 2.7: Narrowband sound spectrogram showing fundamental frequency (F0) and harmonics (H). The cursor shows a frequency of $658 \mathrm{~Hz}$ at 3243 milliseconds (Source: Author)

Frequency and time coordinates can be selected at any point in a spectrogram (Figure 2.7). The long vertical line of the cursor gives the time in milliseconds, while the short horizontal cross bar gives the frequency co-ordinate in $\mathrm{Hz}$ (Miller, 2008). In Figure 2.6, the fundamental frequency and the first nine harmonics are clearly seen. In accordance with the colour scale for loudness, F0 and H2 appear to be the loudest (red) and $\mathrm{H} 9$ the quietest (blue) with the harmonics in between showing intermediate degree of loudness. The wiggle in the harmonics shows vibrato while the breaks in the harmonic line indicate consonants interrupting the vocal line. 


\subsection{Speaking fundamental frequency}

Fundamental frequency $\left(F_{0}\right)$ is the rate at which the vocal folds vibrate. It is mainly determined by their tension and length (Hirano, 1974; Evans et al., 2006; Hamdan et al., 2012) and is measured in Hertz (Hz). Speaking fundamental frequency (SFF) is "the average fundamental frequency in connected speech" (Boone et al., 2010: p. 150). Sometimes the terms $F_{0}$ and SFF are used interchangeably (Colton et al., 2011). For the purposes of this thesis, SFF will be used to refer to the mean $F_{0}$ of a given sample of speech.

There are many influences on SFF including subject selection criteria and subject variables (Table 2). Particularly relevant to this thesis are the person's age, voice type, training and anthropometric indices.

Table 2: Variables influencing SFF

\begin{tabular}{|c|c|}
\hline $\begin{array}{c}\text { Variable } \\
\text { influencing SFF }\end{array}$ & References \\
\hline senescence & $\begin{array}{l}\text { (McGlone \& Hollien, 1963; Honjo \& Isshiki 1980; } \\
\text { Stoicheff, 1981; Alarcos et al., 1983; Mueller et al., } \\
\text { 1984; Linville \& Fisher, 1985; Linville, 1987; } \\
\text { Pegoraro- Krook, 1988; Higgins \& Saxman, 1991; } \\
\text { Russell et al., 1995) }\end{array}$ \\
\hline $\begin{array}{l}\text { professional } \\
\text { training }\end{array}$ & $\begin{array}{c}\text { (McGlone, 1977; Watson \& Hixon, 1985; Morris \& } \\
\text { Brown Jr, 1987; Brown Jr et al., 1988; Brown Jr et al., } \\
\text { 1991; Brown Jr et al., 1993; Baken \& Orlikoff, 2000; } \\
\text { Brown Jr et al., 2000) }\end{array}$ \\
\hline task type & $\begin{array}{l}\text { (Mysak, 1959; Horii, 1975; Hudson \& Holbrook, 1982; } \\
\text { Ramig \& Ringel, 1983; Britto \& Doyle, 1990; Fitch, } \\
\text { 1990; Murry et al., 1995; Zraick et al., 2000; Baker et } \\
\text { al., 2008; Schiwitz, 2011) }\end{array}$ \\
\hline measuring method & $\begin{array}{l}\text { (Hirano, 1989; Morris \& Brown Jr, 1996; Behrman, } \\
\text { 2005) }\end{array}$ \\
\hline
\end{tabular}




\begin{tabular}{|c|c|}
\hline $\begin{array}{l}\text { anthropometric } \\
\text { indices }\end{array}$ & $\begin{array}{l}\text { (Bricker \& Pruzansky, 1976; Lass \& Davis, 1976; } \\
\text { Lass et al., 1977; Lass, Barry, et al., 1979; Lass, } \\
\text { DiCola, et al., 1979; Lass \& Colt, 1980; Graddol \& } \\
\text { Swann, 1983; van Dommelen \& Moxness, 1995; } \\
\text { Kreiman 1997; Krauss et al., 2002; Evans, et al., } \\
\text { 2006; Hamdan, et al., 2012) }\end{array}$ \\
\hline language & $\begin{array}{c}\text { (Hollien \& Malcik, 1962; Kitzing, 1979; Hudson \& } \\
\text { Holbrook, 1982) }\end{array}$ \\
\hline culture & (Harris et al., 1998) \\
\hline race & $\begin{array}{l}\text { (Hollien \& Malcik, 1962; Hudson \& Holbrook, 1982; } \\
\text { Wheat \& Hudson, 1988; Xue \& Mueller, 1996) }\end{array}$ \\
\hline dialect & $\begin{array}{c}\text { (Hanley, 1951; Coleman \& Markham, 1991; } \\
\text { Altenberg \& Ferrand, 2006) }\end{array}$ \\
\hline $\begin{array}{c}\text { hormonal factors } \\
\text { and menstrual } \\
\text { cycle }\end{array}$ & $\begin{array}{l}\text { (Rubin, 1988; Higgins \& Saxman, 1989; Abitbol et al., } \\
\text { 1999) }\end{array}$ \\
\hline $\begin{array}{l}\text { vocal fold oedema; } \\
\text { vocal fatigue }\end{array}$ & (Bagnall et al., 2011) \\
\hline vocal fold flexibility & (Harris, et al., 1998) \\
\hline $\begin{array}{l}\text { intrasubject } \\
\text { variability }\end{array}$ & (Coleman, 1993) \\
\hline personality & (Wolfe et al., 1990; Harris, et al., 1998) \\
\hline emotional status & (Traunmuller \& Eriksson, 1994) \\
\hline smoking & (Gilbert \& Weismer, 1974) \\
\hline stress & (Rubin, 1988) \\
\hline $\begin{array}{l}\text { pathology (organic } \\
\text { and functional } \\
\text { vocal pathology) }\end{array}$ & $\begin{array}{c}\text { (Brodnitz, 1966; Murry, 1978; Murry \& Doherty, 1980; } \\
\text { Hirano, 1989) }\end{array}$ \\
\hline vocal fold nodules & (Peppard et al., 1988) \\
\hline physical conditions & (Drew \& Sapir, 1995) \\
\hline
\end{tabular}


There have been numerous studies on SFF in males and females of all ages. There is considerable variation among reported values and definitive normal levels have not yet been established. However, a typical mean SFF value for a male or female child is approximately $220-300 \mathrm{~Hz}$, for a premenopausal adult woman around $224 \mathrm{~Hz}$ (range 180-250 Hz), a postmenopausal women approximately $200 \mathrm{~Hz}$ (range 160-230), and for an adult male approximately $128 \mathrm{~Hz}$ (range 100-150 Hz) (Fairbanks, Herbert, et al., 1949; Fairbanks, Wiley, et al., 1949; Fairbanks, 1959; Hollien et al., 1971; Hollien \& Shipp, 1972; Aronson, 1980; Stoicheff, 1981; De Pinto \& Hollien, 1982; Sorenson, 1989; Brown Jr, et al., 1991; Traunmuller \& Eriksson, 1994; Russell, et al., 1995; Harris, et al., 1998; Baken \& Orlikoff, 2000; Lawrence, 2004; Lindh, 2006; Shewell, 2009). These figures are generalisations and individual SFF values vary considerably.

It is generally accepted that mean SFF values and ranges change with age. The SFF of girls and boys decreases to adult levels at puberty (Duffy, 1970; Hollien et al., 1994; Harries et al., 1998). In adult women SFF remains relatively stable after adolescence until it decreases at the menopause, stabilising again after the menopause (McGlone \& Hollien, 1963; Chevrie-Muller et al., 1971; Kitzing, 1979; Stoicheff, 1981; Benjamin, 1986; Pegoraro- Krook, 1988; Higgins \& Saxman, 1991; Russell, et al., 1995; Raj et al., 2010). It has been suggested that the observed decrease in SFF in women during their $50 \mathrm{~s}$ is directly related to physiological changes in the larynx associated with changing hormone levels, rather than chronological age (Abitbol, et al., 1999). In males, a dramatic decrease in SFF occurs at puberty. Progressive lowering continues until around 50 years of age, when SFF starts to increase (Mysak, 1959; Hollien \& Shipp, 1972; Kitzing, 1979; Pegoraro- Krook, 1988; Brown Jr, et al., 1991; Traunmuller \& Eriksson, 1994; Decoster \& Debruyne, 1997; Verdonck-de Leeuw \& Mahieu, 2004; Harnsberger et al., 2008). Hollien and Shipp (1972) noted that age related changes in SFF seemed to be less prominent or even absent in trained voice professionals, especially singers, which was subsequently confirmed by others (Brown Jr, et al., 
1991; Morris et al., 1995). Data from the National Center for Voice and Speech in the USA are shown in Figure 2.8.

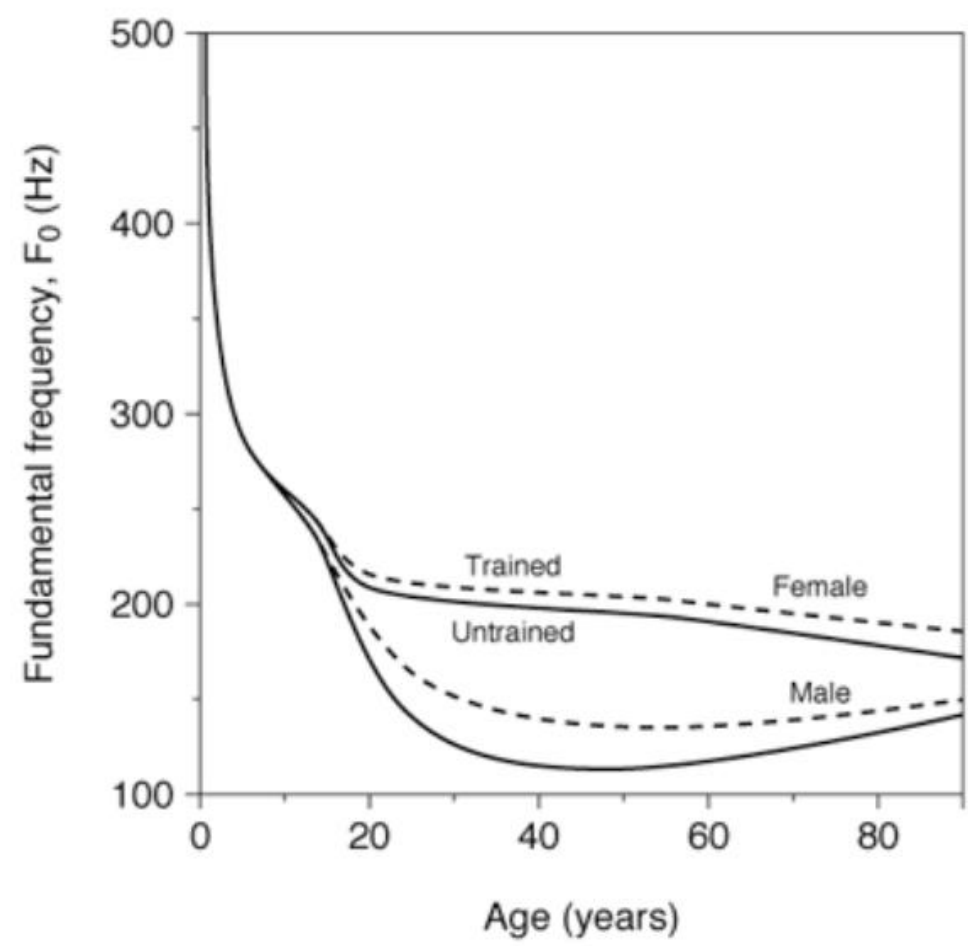

Figure 2.8: Average $F_{0}$ as a function of age for trained and untrained men and women (National Center for Voice and Speech, 2013b; used with permission)

There have been a few studies on SFF in singers (Nadoleczny, 1923; Koufman \& Blalock, 1988; Brown Jr, et al., 1991; Brown Jr, et al., 1993; Drew \& Sapir, 1995; Morris, et al., 1995; Larsson \& Hertegård, 2008) and limited reports of SFF based on voice classification. Even though there is a wide variation in SFF values within each voice category, it has been shown that SFF differs significantly between voice categories: sopranos have the highest SFF and basses the lowest. Comparing recorded voices to tuning fork frequencies Nadoleczny (1923) reported data on average SFF for singers in six different voice categories: basses, $98 \mathrm{~Hz}$; baritones, $116 \mathrm{~Hz}$; tenors, $134 \mathrm{~Hz}$; contraltos, $212 \mathrm{~Hz}$; mezzo-sopranos, $230 \mathrm{~Hz}$ and sopranos, $262 \mathrm{~Hz}$. Similar results were obtained by Roers (2005) who used a digital computer programme to record and measure SFF. In this study, reported average SFF results for the six different voice categories were: basses, $104 \mathrm{~Hz}$; baritones, $116.5 \mathrm{~Hz}$; tenors, $139 \mathrm{~Hz}$; contraltos, $220 \mathrm{~Hz}$; mezzo-sopranos, $220 \mathrm{~Hz}$ and sopranos, $233.5 \mathrm{~Hz}$. Larsson and 
Hertegård (2008) also used recorded voices and a computer programme to measure SFF, yielding average values of $91 \mathrm{~Hz}(78-114)$ for basses, $113 \mathrm{~Hz}(106-124)$ for tenors, $177 \mathrm{~Hz}(153-205)$ for mezzo-sopranos and $189 \mathrm{~Hz}(160-247)$ for sopranos.

SFF data has been collected using spontaneous speech and by asking participants to read a passage and different methods have been used to record SFF. There is no standardised protocol for measuring SFF. Some studies have shown that SFF values for reading tasks are consistently higher than those for spontaneous speech (Snidecor, 1951; Mysak, 1959; Schultz-Coulon, 1975; Horii, 1982; Hudson \& Holbrook, 1982; Ramig \& Ringel, 1983; Fitch, 1990; Traunmuller \& Eriksson, 1994; Schiwitz, 2011). There is some evidence that different measurement techniques result in significantly different results (Horii, 1975; Hudson \& Holbrook, 1982; Britto \& Doyle, 1990; Murry, et al., 1995; Zraick, et al., 2000; Zraick et al., 2005; Baker, et al., 2008; Chen et al., 2009; Hunter, 2009).

Numerous publications have suggested a correlation between SFF and physical characteristics. In particular height, weight and BMI are thought to affect SFF (Evans, et al., 2006). Even Charles Darwin (1871) suggested that there may be a link between $F_{0}$ and body size. Laver and Trudgill (1979) implied a negative correlation between SFF and the height or weight due to their observation that tall, well-built men tend to have a low SFF. A study by Evans et al. (2006) supported this assertion, at least in men with increased upper body musculature, a wide chest circumference and high shoulder to hip ratio. However, several studies contradict this observation (Lass \& Brown, 1978; Graddol \& Swann, 1983; Kunzel, 1989; Collins, 2000; Hamdan, et al., 2012). Overall, studies show no consistent correlation between SFF and body size.

Using a completely different approach, several studies have investigated the ability of listeners to judge the height and weight of the speaker from speech samples. A number of these studies have claimed that listeners can correctly estimate height and weight from voice samples (Lass \& Davis, 1976; Lass, et al., 1977; Lass, Barry, et al., 1979; Lass, DiCola, et 
al., 1979; Lass \& Colt, 1980; Graddol \& Swann, 1983; Krauss, et al., 2002). Conversely, several studies have concluded that from speaker $F_{0}$ neither height nor weight parameters could be estimated reliably by listeners (Gunter \& Manning, 1982; Kunzel, 1989; van Dommelen \& Moxness, 1995; Collins, 2000). According to Gonzales (2003), the studies by Lass and colleagues should be interpreted with caution.

In conclusion, SFF values are affected by age, gender and vocal training. SFF tends to decrease slightly at the menopause in women but gradually increases after 50 years of age in men. There are still conflicting opinions surrounding the suggested correlation of SFF and anthropometric indices. More research is needed to examine the consistency of measuring SFF. 


\subsubsection{Surgical alteration of fundamental frequency}

Vocal pitch can be changed surgically by altering the thickness, length or tension of the vocal folds. Scherer and Guo (1990) developed a formula ${ }^{1}$ which clearly showed that vocal pitch can be altered if one or more of the following can be achieved:

- A change in the tension of the vocal fold (relatively less efficient, since the relationship to $\mathrm{F} 0$ is the square root rather than linear)

- A change in the total mass/density of the vocal fold (also less efficient, because of the square root relationship),

- A change in the vibrating length of the vocal fold (highly efficient).

Current operative techniques for surgical alteration of voice pitch are based on one or more of these principles. In simple terms, $F_{0}$ is inversely proportional to twice the length of the vocal folds, inversely proportional to the square root of their density (mass per unit volume), and directly proportional to the square root of their tension (Colton, 1988; Scherer \& Guo, 1990; Laukkanen et al., 2002; Lawrence, 2004). Vocal pitch can be elevated by various surgical techniques which aim to increase vocal fold tension, shorten the length of the vibratory portion of the vocal folds, reduce total vocal fold mass, or through a combination of these methods (Gross, 1999; Neumann et al., 2002; Lawrence, 2004; Colton, et al., 2011). These techniques have become the standard methods used to raise vocal pitch (Storck et al., 2011), especially in male to female transgender subjects (Yang et al., 2002).

A variety of actual procedures for raising voice pitch have been described. These include cricothyroid approximation, cricothyroidopexy, cricothyroid fixation, cricothyroid subluxation, anteroposterior extension of the thyroid ala, endolaryngeal shortening of the vocal folds, vocal ligament tightening, anterior commissure advancement, thyroid cartilage and vocal fold

\footnotetext{
${ }^{1} F_{0}=(1 / 2 L) \sqrt{ } T / P$ where $L$ is the length of the vocal folds, $T$ is the mean longitudinal tension of the vocal folds and $\mathrm{P}$ is the mass (density) of the vocal folds (Scherer \& Guo, 1990)
} 
reduction, feminising laryngoplasty, vocal fold reduction by laser, vocal fold resurfacing, partial thyroarytenoid myectomy (with or without the laser), scarification, vocal fold stripping, longitudinal incisions in the folds, and injection of steroid into the vocal folds. For a more detailed explanation of these procedures see Appendix A.

Two of the more common methods are Cricothyroid Approximation and Anterior Commissure Laryngoplasty. Cricothyroid Approximation (CTA) is a procedure in which the CT space is reduced by approximating the cricoid and thyroid cartilages (Figure 2.9). This increases vocal fold tension (Lawrence, 2004) and elevates vocal pitch (Isshiki, 1989; Neumann, et al., 2002).
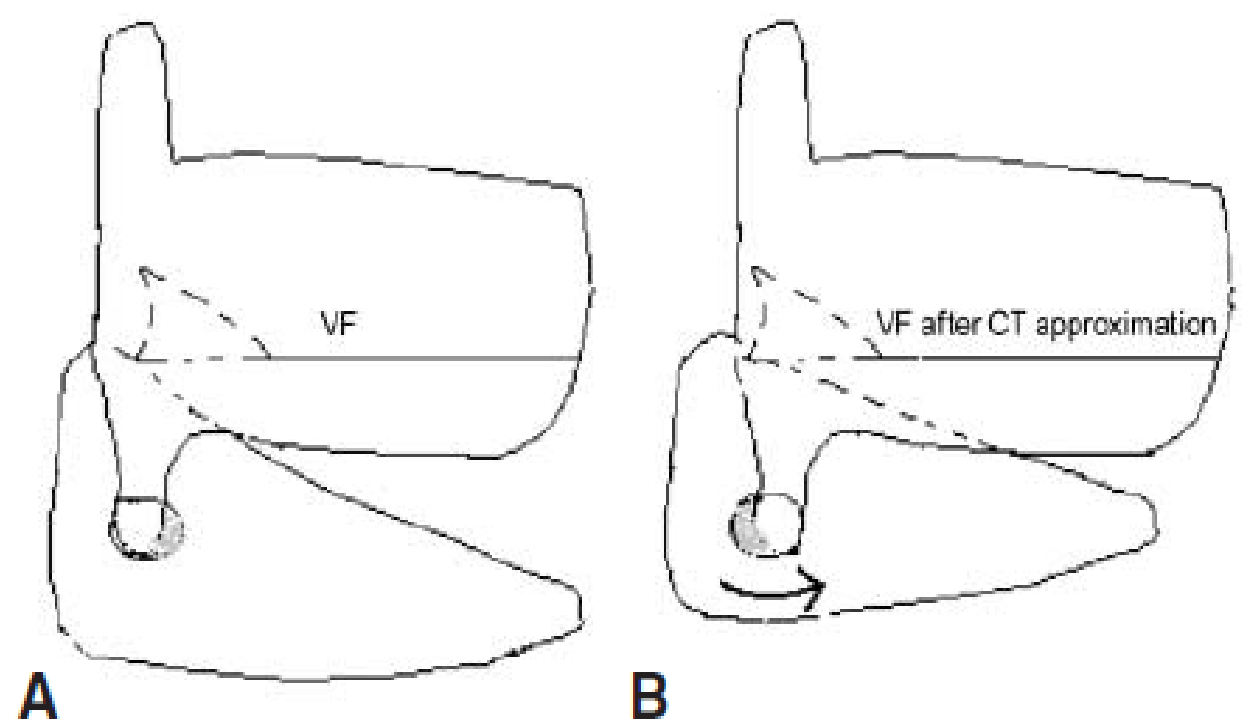

Figure 2.9: Schematic representation of CTA. A: shows the position of the cricoid and thyroid cartilages and vocal folds (VF) at rest. B: approximation of cricoid and thyroid cartilages and position of vocal folds after CTA (Filho et al., 2005; used with permission)

Anterior Commissure Laryngoplasty is the second most popular technique to increase $\mathrm{F}_{0}$. Two main methods are employed: creation of an anterior web thereby shortening the vibratory length of the folds (Donald, 1982; Wendler, 1990; Gross \& Fehland, 1995; Gross, 1999) (Figure 2.10), and 
anterior commissure advancement which increases vocal fold tension by stretching (LeJeune et al., 1983; Tucker, 1989).
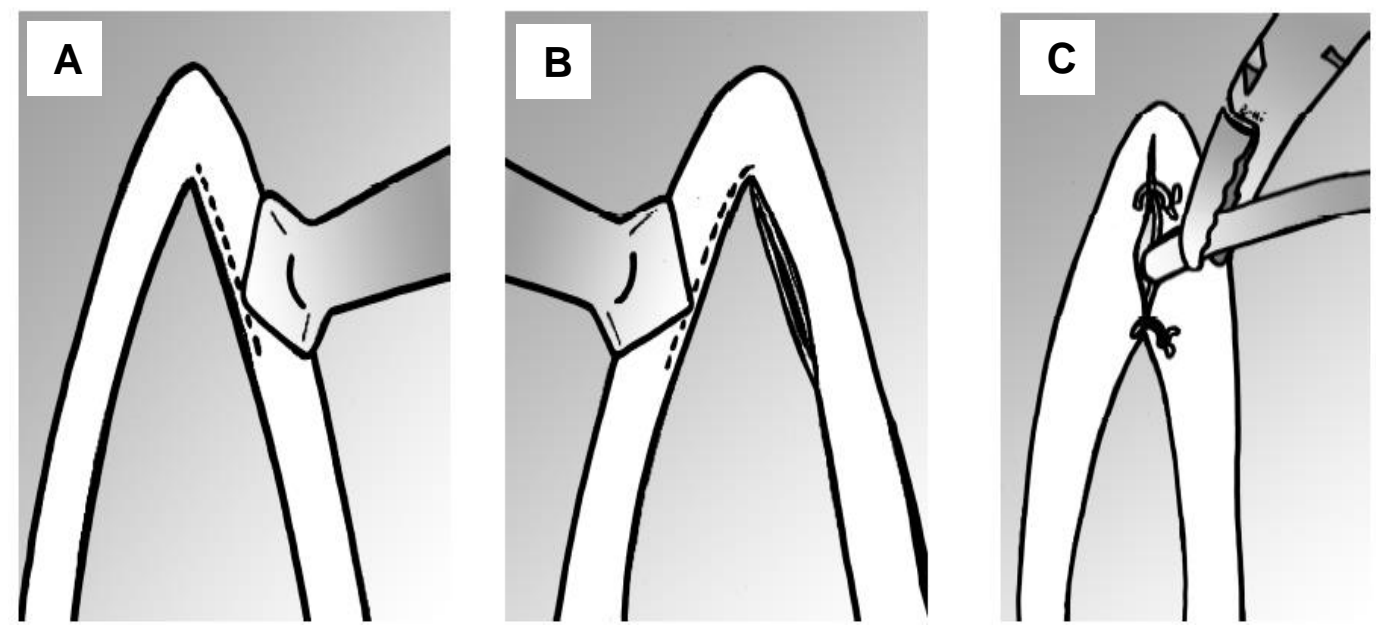

Figure 2.10: Anterior Commissure Laryngoplasty A and B: decortication of the anterior third of the vocal fold C: suturing of anterior thirds of the vocal folds together to reduce the vibratory portion of the folds (Ricci-Maccarini et al., 2011; used with permission)

Techniques used to surgically lower $F_{0}$ can be classified as Relaxation Laryngoplasty (Friedrich et al., 2007) (Figure 2.11). The techniques are Type III thyroplasty and anterior commissure retrusion. The main aim is to shorten the distance between the vocal fold attachments, thereby reducing the tension of the vocal folds and causing pitch to decrease (Isshiki et al., 1974).
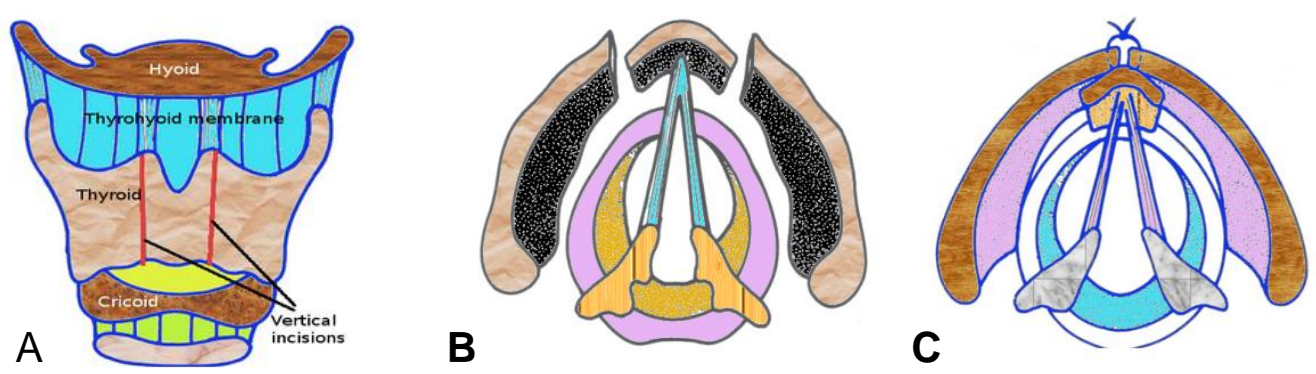

Figure 2.11: Relaxation Laryngoplasty (Isshiki Thyroplasty III). A: Shows vertical incision lines on the thyroid cartilage, $B$ : shows the depressed anterior segment of the thyroid cartilage, C:shows the anterior segment of the thyroid cartilage which has been pushed posteriorly and the free edges of the thyroid cartilage reapproximated anteriorly (Balasubramanian, 2010) 


\subsection{Vocal range and voice classification (Type)}

Simply defined, vocal range is the span from the lowest to the highest note that a singer can produce (Mills, 2012). However, not all pitches in the total vocal range that an individual voice can physically produce may be sung effectively. The range of pitches with a sound of acceptable quality is usually smaller than the physiological frequency range. This range of lowest to highest notes is often referred to as the singer's 'performance range', and is normally used to describe a singer's vocal range.

There are three major voice categories for females, each associated with the following vocal ranges: (Note middle $\mathrm{C}=\mathrm{C} 4$ )

Soprano: $\quad \mathrm{C} 4-\mathrm{C} 6$

Mezzo-soprano: A3-A5

Contralto: $\quad$ F3 - F5

This is a broad generalisation. Singers may be able to sing higher or lower than their assigned voice type (Greene, 1972), they may lie between the typical ranges of two categories, or possess a vocal range that entirely encompasses two voice types (O'Connor, 2012). Appendix B lists specific ranges of sub categories relevant to this thesis.

Determination and alteration of pitch within the vocal range, including extremes of high and low (Miller, 1986b), requires functional adjustment of the structures involved in voice production (Hammer et al., 2010; Mürbe et al., 2011), and the coordinated action of intrinsic and extrinsic laryngeal muscles (Atkinson, 1978). Vocal fold length, which is controlled by the cricothyroid and thyroarytenoid muscles, is a key parameter affecting the pitch range of the voice (Hong et al., 1998; Roers et al., 2009). To be able to achieve the range of frequencies required for singing, the length of the vocal folds must undergo dramatic changes in their length and tension (Hammer et al., 2010). The vocal folds must be long, thin and taut to produce high frequencies; conversely, producing low frequencies requires short, thick and relatively slack vocal folds. The control and coordination of the muscles involved in effecting these alterations of the vocal folds can 
be enhanced by training and mastery of vocal technique (Jiang, et al., 2000; Colton, et al., 2011; Mürbe, et al., 2011).

A major goal of every singer is to have as well-developed and as full a range as physically possible. Maintaining an even timbre throughout the vocal registers (head, middle, and chest voice) is also a major aim in classical voice training (Miller, 1986b). Once a good technique has been established within a limited and comfortable range of pitches, then the range can be increased slowly in both directions incrementally, making sure vocal quality is preserved. Studies have shown that singers have a wider vocal range than non-singers (Åkerlund et al., 1992; Brown Jr, et al., 1993) and both performance and total physiological range can be extended, particularly at the higher end of the frequency range (Mendes et al., 2003).

The vocal range may be limited by poor vocal technique, (Bastian, 1996). However, some singers may still not reach their full potential range (Miller, 1986b), even with good technical coordination and healthy vocal production. There are many reasons for this, all of which limit vocal freedom, particularly in the upper register. These include: lack of energy, anxiety about top/bottom notes, jaw problems (tight, locked, downward projecting or a pushed out forward jaw), tongue problems (tight or tense), facial problems, mouth and/or lip problems (lip tension, upper lip pulled down over top teeth, protruding or pursing of lips), lowered soft palate, lowered cheek muscles under the eyes and nose, a closed throat, lack of air flow, and an elevated or depressed larynx (Miller, 1986a; O'Connor, 2012).

A restricted pitch range is a characteristic feature of vocal fold pathology e.g. nodules, polyps, cysts, haemorrhage etc. The presence of a lesion impedes the laryngeal adjustments necessary for production of a full pitch range. When the pathological condition resolves, a full pitch range is usually regained (Colton, et al., 2011). Some surgical procedures such as CT approximation (Yang, et al., 2002), CT fixation (Sataloff et al., 1992) 
and CT resection (Smith et al., 2008), may also decrease the highest attainable pitch and therefore the vocal range.

Even with vocal training and exercises, the voice will always have an upper and lower pitch limit (Miller, 2000). Each voice is unique. Some singers will be able to develop exceptionally broad ranges, while others have a narrower range (O'Connor, 2012). An individual's vocal range is partly dependent on their unique anatomical and physiological vocal apparatus (Bastian, 1996; Pedersen \& McGlashan, 2010; Mürbe, et al., 2011).

\subsubsection{Senescence and vocal range}

Unfortunately, voices do not improve with age. It is not possible to pinpoint the start of vocal decline with ageing as many factors are involved including psychological health, hereditary and social factors (Behlau et al., 1988; Colton, et al., 2011). All parts of the vocal tract are affected by ageing (Sataloff et al., 1997) and the processes are diverse. Changes such as atrophy (loss of tissue such as muscle mass), reduced elasticity, and oedema (swelling due to excessive accumulation of tissue fluid) can affect the larynx. Specifically, the vocal folds may thicken, the superficial layer of the lamina propria may become thinner and/or more oedematous, and the vocalis muscle may atrophy. The main effect on the voice is a change in vocal range, with progressive loss of top voice, expansion of lower voice, and reduction in total voice range (Mueller, et al., 1984; Linville, 1987; Sataloff, et al., 1997; Teles-Magalhães et al., 2000; Verdonck-de Leeuw \& Mahieu, 2004). Other effects of aging include a deterioration in vocal quality, abnormal vibration of the vocal folds, reduction in the control of pitch, a marked decrease in vocal agility, reduced responsiveness between nerve impulses and muscle fibres (adversely affecting the cricothyroid and thyroarytenoid muscles), a decrease in glottal firmness and reduced elasticity of the vocal folds. 
The laryngeal cartilages can also undergo calcification or ossification with advancing age (Von Leden, 1977; Honjo \& Isshiki 1980; Kahane, 1994). Paradoxically, this may improve the singing voice by providing a more rigid framework to the larynx and vocal folds. However, excessive ossification renders the larynx too rigid, impeding relative movement between the cartilages, especially if ossification takes place in or around the cricothyroid joints or if the cartilages actually fuse together (Kahane, 1994). The cartilages of the larynx may begin to calcify and lose elasticity after 25 years of age but, at the other end of the spectrum, Zenkler (1960) noted that the thyroid cartilage may still be elastic at the age of 70 years.

Women have additional hormonal issues in relation to senescence and vocal range. In women, the hormones oestrogen, progesterone and testosterone are a major factor in determining changes in the larynx and the voice throughout life. Abitbol et al. (1989) showed that hormonal changes in women after the menopause caused the vocal folds to become less supple, with a thinner mucosa and decreased vibratory amplitude. This was reflected by greater vocal fatigue and a decrease in vocal range with loss of high notes and an increase in lower notes. Subtle changes can occur during the menstrual cycle (Rubin, 1988; Higgins \& Saxman, 1989).

Regular vocal training, physical fitness and maintenance of mental health may slow the rate of decline (Sataloff, et al., 1997). Regular vocal exercises help to keep the laryngeal cartilages flexible, extending the life of the singing voice. In trained singers, age related changes seem to be less prominent or even absent (Hollien \& Shipp, 1972; Sataloff, et al., 1997). The well-known Italian tenor, Martinelli, was still singing roles at the Metropolitan Opera House at the age of 76 years. His final professional role was Emperor Altoum, in Puccini's opera Turandot, at the advanced age of 82 years (Greene, 1972). 


\subsubsection{Voice classification}

Voice type is classified on the basis of several qualities or characteristics (Shewan, 1979). These include vocal range, tessitura, timbre (colour), vocal register transition points (passaggio), vocal weight, and, to a lesser extent, speaking fundamental frequency (SFF), and physical characteristics (Chagnon, 1998).

As expected, vocal classification relies significantly on the lowest and highest useable notes of the performance range (Mendes, et al., 2003). Sopranos have the highest useable notes while basses have the lowest. Vocal tessitura refers to the range over which the singer is most comfortable. This is generally where the voice has the best tone quality and pleasing sound. Timbre or colour simply refers to the quality of the tone produced by the singer. This is influenced by the shape and volume of the vocal resonating chambers. Passaggio is the term used to describe the frequency/pitch range at which the voice shifts into the next vocal register. There are two vocal registration areas, lower and upper passaggio, dividing the three accepted timbres of chest, middle and head voice. In classical singers, vocal weight refers to the perceived 'lightness' or 'heaviness' of a singing voice. A heavy voice is often associated with the term 'dramatic'; it tends to be powerful, dark and rich. At the other end of the spectrum is the lighter 'lyric' voice, which has an agile bright style in classical performance. A soprano voice is often considered to be a lighter voice, but this is a misconception, as some soprano voice types, such as a dramatic coloratura soprano, have an intermediate vocal weight. Vocal weight is often a factor when composing because it can affect a performance in many ways. The heavier voice can be used to fill a dramatic role with a large orchestra, as it can carry over a large ensemble better than a lighter voice. In contrast, because of its agility, a lighter voice can more easily negotiate florid coloratura passages (O'Connor, 2012).

SFF is another criterion that may be used to classify singers. Sometimes, it has implications for decision making in relation to vocal performers (Moses, 1949; Cleveland, 1977). If a soprano speaks at too low a pitch, 
there is a possibility that the voice may be classified as a Mezzo-soprano or lower (Drew \& Sapir, 1995).

The German Fach system is a method of classifying singers, according to the range, weight and colour of their voices. Vocal anatomy and function determine a singer's Fach. Although it is possible for an individual to alter the functionality of their voice through vocal training, Fach type is largely determined by inherited anatomical and physiological characteristics (Miller, 1986b). In operatic works some roles can be sung by more than one Fach type. Many singers are not easily categorised into a single Fach type and will often be a hybrid of two Fach types (Miller, 2000).

Twenty five standard Fach categories are recognised with some additional specialised categories defined for specific operatic roles (Appendix C). Classical singers are divided into the six basic vocal categories of Bass, Baritone and Tenor for men, and Alto, Mezzo-Soprano and Soprano for women. The Fach system then further subdivides the type of each voice within each group (Suverkrop, 2013). For example, the variety of soprano is further classified as coloratura, dramatic coloratura, soubrette/coloratura, soubrette, lyric, lyrico spinto, spinto, dramatic or young dramatic, or the variety of mezzo-soprano as dramatic or lyric. In many instances there are only minor differences separating each category (McGinnis, 2010). 


\subsubsection{Relationship between voice type and anatomical features}

Although it can be modified by technique and training, voice type is largely determined by the size and structure of the larynx (although knowledge of laryngeal anatomy cannot precisely define voice type) and the physical characteristics of the individual. Thus, the length and mass of the vocal folds (Titze, 1994) and their configuration, vocal tract dimensions including the size and shape of the resonating oral cavities (Sundberg, 1987), and the physical size and structure of the larynx (Sataloff, 1995) all influence the voice along with body size and shape.

The physical characteristics of an individual such as height, weight, and neck circumference can sometimes predict the type of voice they have (Roers et al., 2007), but there are no definitive correlations. Previous observations of male singers suggest that basses and low baritones are often tall and tend to have long thin necks (Doscher, 1994; Larsson \& Hertegård, 2008), while tenors are shorter and have short, broad necks (Jewett, 1869; Larsson \& Hertegård, 2008). The link between neck length and size and voice type is less obvious in females (Larsson \& Hertegård, 2008), but sopranos tend to be shorter individuals than mezzos. Jewett (1869) suggested that sopranos have a short neck and high shoulders, while contraltos have a very long neck and sloping shoulders. Many coloratura sopranos have a relatively small body and head and a shorter neck (Doscher, 1994). Mezzo-sopranos are typically women with a particularly symmetrical neck and shoulders (Jewett, 1869).

In a review of laryngeal morphology and voice classification, Murbe et al., (2011) concluded that voice type is related to body height, vocal fold length, and vocal tract length. Generally, the taller the person, the longer their vocal folds and vocal tract, which in turn affects the primary qualities or determinants of their voice. This same group of researchers previously established a direct relationship between the dimensions of the vocal folds (length, width, and mass) and voice classification (Roers, et al., 2009). It is known that males have longer vocal folds than females (Nishizawa et al., 1988; Hertegård et al., 1993; Su et al., 2002; Schuster et al., 2005; 
Larsson \& Hertegård, 2008; Mürbe, et al., 2011). However, there are also differences in vocal fold length between voice types e.g. between soprano and mezzo-soprano, or tenor and bass (Larsson \& Hertegård, 2008). Generally, the greater the dimensions of the vocal folds, the lower the voice category. Conversely, smaller dimensions of the vocal folds are consistent with higher voice types (Lawrence, 2004). To quote Brodnitz (1965) "...the long cords of a man with their greater mass produce a deeper sound than the shorter cords of a women..." (p. 21).

As a general rule, singers with larger vocal tracts and greater vocal fold dimensions have lower passaggio pitch areas, and lower ranges and tessituras. Sopranos tend to have the shortest vocal tracts and basses the longest and the voices are perceived as darker (Chagnon, 1998). Conversely, singers with smaller vocal tract dimensions have higher passaggio pitch areas, ranges and tessituras. Adult males tend to have a longer vocal tract than adult females (Chiba \& Kajiyama, 1941; Roers, et al., 2009) and the adult male larynx is generally more caudally positioned (Balasubramanian, 2010). The distinctive timbre or resonance of the male voice is primarily due to the greater length of the upper airway (throat, mouth, nose, and sinuses (Fitch \& Giedd, 1999). A quote by Laver and Trudgill (1979) succinctly states:

A tall well-built man will tend to have a long vocal tract and large vocal folds. His voice quality will reflect the length of his vocal tract by having correspondingly low ranges of formant frequencies, and his voice dynamic features will indicate the dimensions and mass of his vocal folds by a correspondingly low range of fundamental frequency (p. 9).

The two principle resonating cavities are the pharynx (which includes the naso-, oro-, and laryngopharynx) and the mouth (oral cavity) (Figure 2.12). With training, the singer can consciously change the dimensions of the resonating system by adjusting the position of the larynx, the shape of the pharynx, the position of the tongue, soft palate, and mandible and the 
shape of the lips (Callaghan, 2000). Raising or lowering the larynx or pursing the lips can alter the length of the vocal tract, and having a longer vocal tract makes the tone darker (Chagnon, 1998). The soft palate and the larynx have an opposing relationship: the arch of the soft palate descends as the larynx rises producing a darker tone, and vice-versa to produce a brighter tone. Gutzmann (1949) found that there were certain tendencies within voice types: a high palate was seen more often in sopranos while a flat wide palate was characteristic of basses. He also noted that a high palate was most common in singers with a light timbre, while a dark timbre was mostly associated with a flat palate.

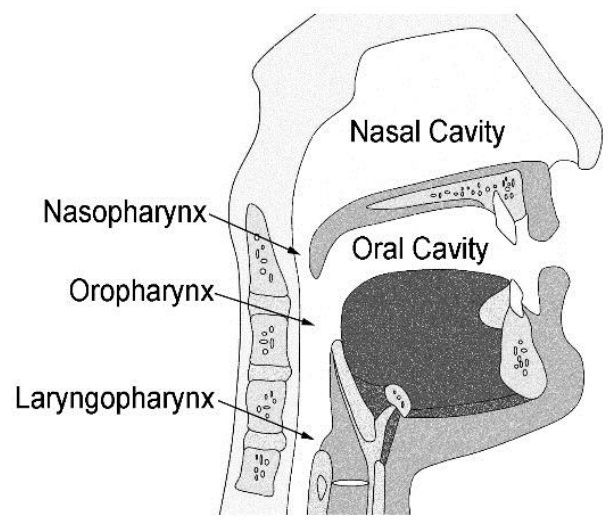

Figure 2.12: Principle resonating cavities; pharynx and oral cavities (McCoy, 2004; used with permisssion)

There is debate about whether the paranasal sinuses and the nasal cavity contribute to resonance and a bright tone in the voice. It is well recognised that when singers develop upper respiratory tract infections and the paranasal sinuses become fluid filled, the quality and the tone of the voice are altered. However, Vennard (1967) stated "...it is very unlikely that the paranasal sinuses impart resonance to the voice... and exert any influence upon vocalisation..." (p. 96). This was subsequently confirmed by Yanagisawa et al. (1989). With regards to nasal cavity resonance, Alderson (1993) asserted "... directing a portion of the sound waves through the nasal cavity adds a more brilliant ring to the tone..." (p. 26). More research is needed to clarify the importance of their role in contribution to resonance. 


\subsubsection{Importance of vocal range and voice classification}

Identification of the correct natural singing range and accurate vocal classification is of crucial importance for a singer. There are numerous risks to the voice if this is compromised, as stress and strain on the voice will lead to vocal fatigue, serious injury to the vocal folds and, ultimately, a voice disorder (Roers et al., 2009). Notes on the outer limits of the range also need to be produced accurately and not over worked, otherwise injury can occur even in trained singers (Greene, 1972; Mürbe, et al., 2011).

Vocal range and correct classification also need to be established for a singer's repertoire selection. For opera singers in particular, this determines what roles are suitable to perform and minimises the risks of damaging the vocal apparatus from singing unsuitable repertoire (O'Connor, 2012). Range is not the only determinant when evaluating a voice but a broader range expands repertoire choices and gives the singer more versatility. As ranges are precisely prescribed within categories, having a limited range is a serious disadvantage to the performer (Miller, 1986b).

If singers are classified too early, they might be classified incorrectly due to limitations in voice range from inexperience, immaturity or temporary technical difficulties. This not only limits their potential but may also cause voice problems. It is often underestimated how much an untrained or novice singer can increase their vocal range and therefore they should not limit themselves to a particular category until all avenues and techniques of training have been explored (Chagnon, 1998; O'Connor, 2012). 


\subsection{The 'gifted voice' and genetic aspects}

The "gifted", "golden", "elite", or "premiere" voice, as referred to by Hollien (1993), is a voice with a "breath-taking" quality. Thus far, there is no general agreement on the morphological characteristics of the larynx in an individual with an outstanding voice. It remains controversial whether the genetically determined anatomical dimensions of the larynx lead to a gifted singer or whether training and dedication are more important.

Variation in the anatomical dimensions of the larynx have been thought by some researchers to have the major influence on the vocal range and voice quality (Miller, 1986b; Sataloff, 1995; Titze, 1998b, 1998a). As Callaghan (2000) stated 'It is commonly believed that premier/elite singers must be physiologically gifted' (p.25). The advent of the laryngoscope has permitted easy access to inspect the larynx. This has been used to assess and determine if any morphologically visible features predict voice quality.

The internationally acclaimed English contralto Kathleen Ferrier was noted to have had a spacious pharyngeal cavity (Henderson, 1954) leading to the suggestion that capacious resonating chambers with a wide opening to the laryngeal vestibule were associated with a naturally good voice (Arnold, 1962; Sundberg, 1987; Sonninen et al., 1999). Titze (1998a) suggested that symmetry of the larynx, including symmetry of the vocal folds, could contribute to normal vocal fold vibration and better control of pitch, loudness and onset. Arnold (1962) suggested that a symmetrical, unobstructed, and easily viewable larynx were features contributing to a good singing voice. Conversely, an asymmetrical, constricted, and poorly accessible larynx would be found in individuals with an inferior singing voice.

Using nasal endoscopy to examine/photograph and videotape larynges of many "gifted", "golden", "elite", or "premiere" singers, Cleveland (1991) reached a different conclusion. He discovered that there was nothing anatomically unique about the visual morphology of the larynx that distinguished these singers from the average singer. The range of 
morphological features, such as obstructions and asymmetry, were no different to those observed in the general population. Based on his research, he concluded that each larynx is "...original, and its potential lies far beyond its appearance" (Cleveland, 1991; p. 51).

For the singer, reasonably normal anatomy is essential, though it appears this is not enough by itself (Greene, 1972; Sataloff, 1995). An individual's voice is determined by much more than genetics and anatomy. Debruyne et al. (2002) suggested that there is a complex interaction between genotype and training. Further research in the very complex subject of genetic influences on voice are needed before it can be determined whether inherited morphology or training is the most important single factor for an exceptional voice (Sataloff, 1995). However, whether the "gifted" voice is innate or learnt, it is still special when singing (Hollien, 1993). 


\subsection{Anatomy of the larynx}

The larynx, or voice box, is placed at the upper part of the air passage, situated between the trachea and the root of the tongue. The larynx houses the two vocal folds (left and right) which are stretched horizontally across the larynx from front to back and create the voice sound by vibrating (Spalding, 1995).

\subsubsection{Laryngeal Cartilages}

The laryngeal skeleton is composed of nine cartilages, three single and three paired cartilages, all inter-connected by ligaments and moved by numerous muscles (Figures 2.13 and 2.14).

- Thyroid

- Cricoid

- Two Arytenoid

- Two Corniculate

- Two Cuneiform

- Epiglottis

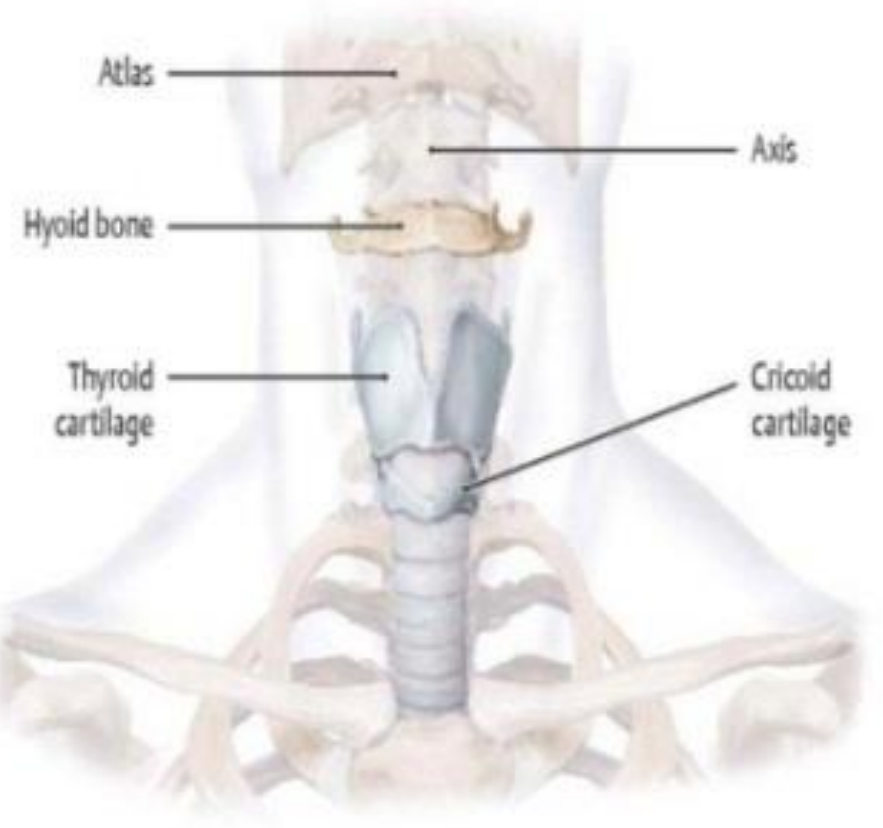

Figure 2.13: Larynx A: Anterior view (Schünke et al., 2006; used with permission) 


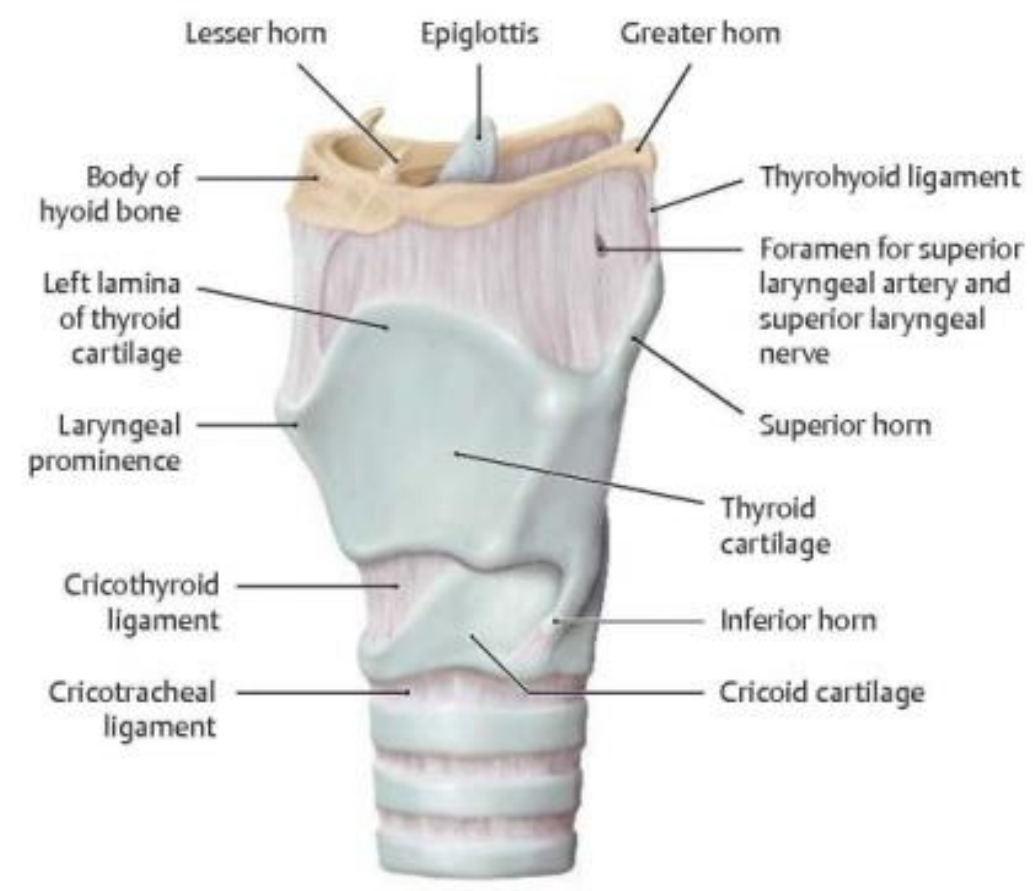

Figure 2.14: Larynx B: Left anterior oblique view (Schünke, et al., 2006; used with permission)

- Thyroid Cartilage

The thyroid cartilage is the largest of the laryngeal cartilages and is shaped like a shield with two laminae that are fused in the midline of the neck to form a laryngeal prominence known as the Adams apple. Situated above this is the distinctive $\mathrm{V}$ shaped notch, the superior thyroid notch. This prominence is usually larger in males than in females. The thyroid cartilage is hinged so that it can move slightly in both a forward and downward motion. Its superior border is connected to the hyoid bone by a membrane and the inferior border articulates with the cricoid cartilage (Spalding, 1995).

- Cricoid cartilage

The cricoid cartilage is a complete ring of hyaline cartilage that is smaller, thicker and stronger than the thyroid cartilage; it forms the inferior limit of the larynx. It is attached to the inferior border of the thyroid cartilage by the cricothyroid ligament (also called the cricothyroid membrane) and is connected to the superior border of the first tracheal ring (i.e. the 'top' of the trachea) (Rosen \& Simpson, 2008). 
- Arytenoid cartilage

The arytenoid cartilages are located at the posterior, superior border of the cricoid cartilage. These paired cartilages are critically important because they effect changes in the position and tension of the vocal folds. Pitch is controlled by the tension in the vocal folds (also known as the vocal cords). The arytenoid cartilages have the ability to rock, glide and pivot and are under the control of the laryngeal muscles. They therefore control the opening and closing of the vocal folds in breathing and phonation (Tortora \& Derrickson, 2006) (Figure 2.15)

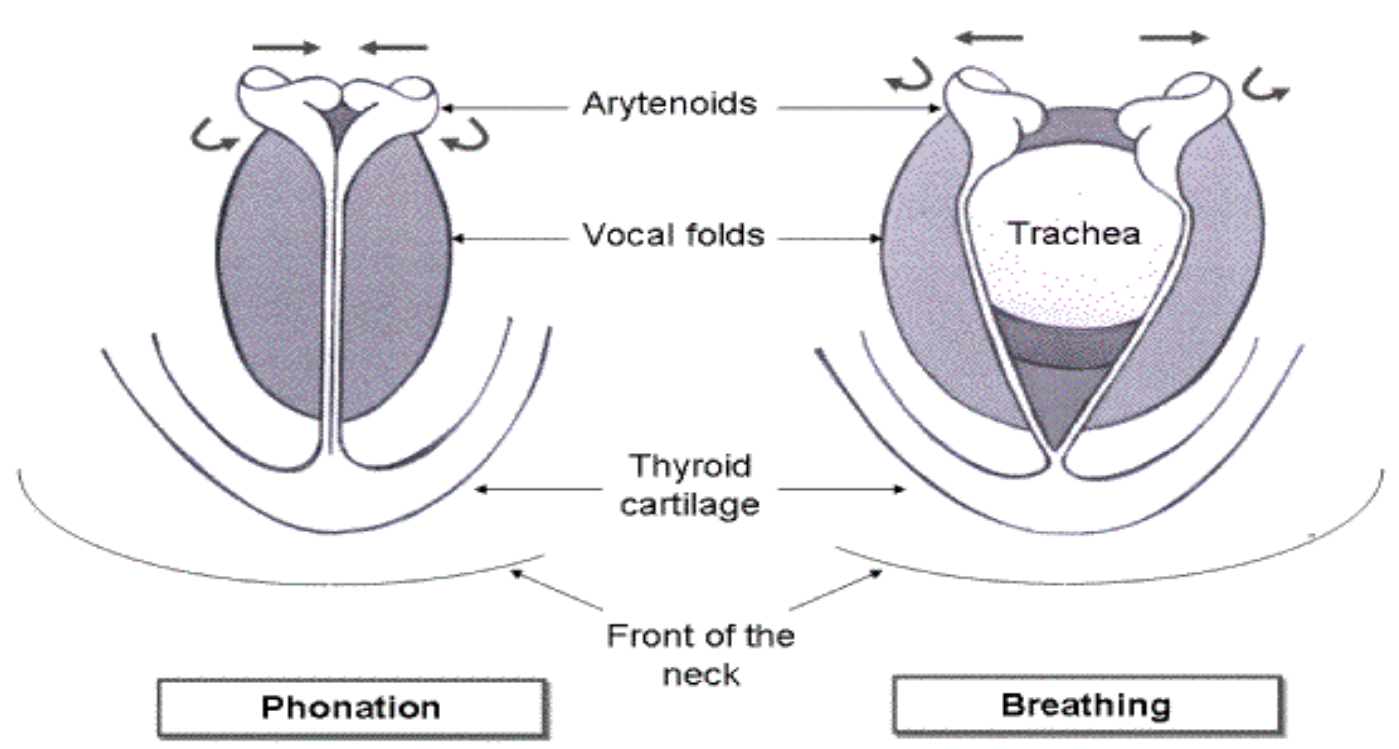

Figure 2.15: Arytenoid cartilage movement (Wolfe, et al., 2010; used with permission)

- Corniculate cartilages

These small paired corniculate cartilages are horn shaped pieces of fibroelastic cartilage located at the apex of each arytenoid cartilage ((Figure 2.16). They are completely embedded within the aryepiglottic folds and are supporting structures for the epiglottis (Rosen \& Simpson, 2008). 


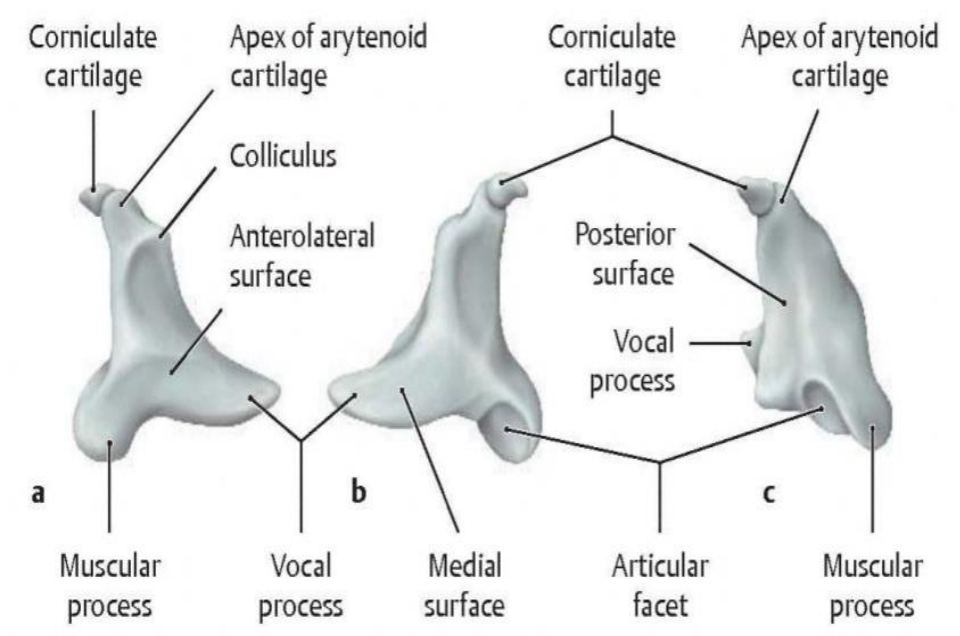

Figure 2.16: Arytenoid and corniculate cartilages. (a) lateral view; (b) medial view; (c) posterior view (Schünke, et al., 2006; used with permission)

\subsubsection{Laryngeal dimensions}

With the advent of sophisticated medical imaging equipment (ultrasound, $\mathrm{CT}, \mathrm{MRI}$, etc), just about any dimension of the human larynx can be accurately measured. The list includes the dimensions of the laryngeal cartilages, internal and external diameters of the cricoid cartilage, height and length of the thyroid alae, angulation of the thyroid laminae, height of the arytenoid cartilages, width and length of the epiglottis (Eckel et al., 1994; Eckel \& Sittel, 1995; Sprinzl et al., 1999; Tayama et al., 2001), and vocal fold dimensions.

Until puberty, there are no significant differences in the size of the larynx between males and females but after puberty, the male larynx becomes considerably larger (Kahane, 1978; Titze, 1988, 1994). It has also been noted that the larynx is relatively more caudally positioned in men compared to women and children (Balasubramanian, 2010). Gender differences in laryngeal dimensions are important for biomechanical modelling and they also have clinical implications (Tayama, et al., 2001). Numerous data on laryngeal dimensions from autopsies can be found in older textbooks of anatomy but, there is no information on how these measurements were taken or precisely where they were measured 
(Sappey, 1874; Behnke, 1900; Testut \& Jacob, 1921; Judson \& Weaver, 1942; Negus, 1949; Lanz \& Wachsmuth, 1955; Gray, 1959). In recent years, several studies have reported detailed measurements of biomechanically important morphometric features of the adult human larynx of both sexes (Table 1) (Eckel, et al., 1994; Eckel \& Sittel, 1995; Tayama, et al., 2001). However, all these measurements were made in cadavers and may not represent actual dimensions of living tissues.

Table 3: Dimensions of the adult larynx in cadavers

\begin{tabular}{|c|c|c|c|c|}
\hline & \multicolumn{2}{|c|}{ Adult males } & \multicolumn{2}{|c|}{ Adult females } \\
\hline & $\begin{array}{l}\text { Dimension } \\
(\mathrm{mm})\end{array}$ & $\mathrm{n}$ & $\begin{array}{l}\text { Dimension } \\
(\mathrm{mm})\end{array}$ & $\mathrm{n}$ \\
\hline $\begin{array}{l}\text { Total Length: }{ }^{2} \\
\text { distance from lower edge of cricoid to } \\
\text { cranial edge of hyoid }\end{array}$ & $\begin{array}{c}63.1 \pm 4.9 \\
(47-71)\end{array}$ & 28 & $\begin{array}{c}51.2 \pm 3.5 \\
(41-58)\end{array}$ & 25 \\
\hline $\begin{array}{l}\text { Transverse distance: }{ }^{2} \\
\text { distance between posterior ends of the } \\
\text { greater cornua of the thyroid cartilage }\end{array}$ & $\begin{array}{c}38.2 \pm 7.7 \\
(27-63)\end{array}$ & 28 & $\begin{array}{c}36.9 \pm 6.4 \\
(26-48)\end{array}$ & 25 \\
\hline $\begin{array}{c}\text { A-P length: }{ }^{3} \\
\text { distance from laryngeal prominence to } \\
\text { posterior edge of larynx at level of } \\
\text { vocal folds }\end{array}$ & $37.3 \pm 4.5$ & 6 & 28.9 & 2 \\
\hline $\begin{array}{l}\text { Internal transverse diameter } \\
\text { of the caudal edge of the cricothyroid } \\
\text { cartilage }^{2}\end{array}$ & $\begin{array}{c}18.2 \\
(13-24)\end{array}$ & 28 & $\begin{array}{c}14.5 \\
(10-17)\end{array}$ & 25 \\
\hline
\end{tabular}

${ }^{2}$ Eckel et al. (1994)

${ }^{3}$ Tayama et al. (2001) 
Ethnic differences have also been investigated in Europeans (Chievitz, 1882; Balboni, 1955; Minnigerode, 1955; Malinowski, 1967; Eckel, et al., 1994), North Americans (Maue \& Dickson, 1971), Black Africans (Ajmani, 1990) and Asian Indians (Longia \& Saxena, 1980; Harjeet \& Jit, 1992; Jain $\&$ Dhall, 2008). Jain and Dhall (2008) found significant positive correlations between an individual's stature and the length of their thyroid lamina, and the height and transverse diameter of their cricoid ring. However, no such correlations were found by Ajimani (1990) and Longia (1990) in Africans and Turkish people respectively.

The measurements most relevant to this thesis are the anterior vertical height of the thyroid and cricoid cartilages in the midline and the vertical dimension of the cricothyroid space. Values for these parameters recorded in previous studies are listed in Table 4. Although these measurements were reportedly similar, there are wide variations in the data. This is particularly obvious in the results of Maue and Dickson (1971), who may have used different sites of measurement. In spite of differences in the methods and number of specimens, some conclusions can still be inferred. In general, significant ethnic differences in the height of the cricoid and thyroid cartilages were found. Not surprisingly, these dimensions were greater in males. Despite the paucity of data, it appears that the anterior vertical CT space is also greater in males. 
Table 4: Comparison of cricoid and thyroid cartilage dimensions in different ethnic groups

\begin{tabular}{|c|c|c|c|c|c|c|c|c|c|c|c|}
\hline \multirow[t]{2}{*}{ Author } & \multirow[t]{2}{*}{$\begin{array}{l}\text { Study } \\
\text { sample } \\
\text { origin }\end{array}$} & \multirow[t]{2}{*}{ Method } & \multirow[t]{2}{*}{$\mathrm{n}$} & \multicolumn{2}{|c|}{$\begin{array}{l}\text { Anterior thyroid vertical height } \\
\text { in midline }(\mathrm{mm}) \pm \text { S.D. }\end{array}$} & \multicolumn{2}{|c|}{$\begin{array}{l}\text { Cricoid arch height } \\
\text { in midline }(\mathrm{mm}) \pm \text { S.D. }\end{array}$} & \multicolumn{2}{|c|}{$\begin{array}{l}\text { Cricothyroid space } \\
\text { vertical space } \\
(\mathrm{mm}) \pm \text { S.D. }\end{array}$} & \multicolumn{2}{|c|}{$\begin{array}{l}\text { Total height of } \\
\text { cartilages } \\
(\mathrm{mm})\end{array}$} \\
\hline & & & & Male & Female & Male & Female & Male & Female & Male & Female \\
\hline $\begin{array}{l}\text { Chievitz } \\
(1882)\end{array}$ & European & Cadavers & $\begin{array}{c}270 \\
135 \text { male } \\
135 \text { female } \\
\end{array}$ & 21.5 & 15.8 & 8.1 & 6.8 & & & & \\
\hline $\begin{array}{l}\text { Maue \& } \\
\text { Dickson } \\
(1971)\end{array}$ & $\begin{array}{c}\text { North } \\
\text { American }\end{array}$ & Cadavers & $\begin{array}{l}10 \text { male } \\
10 \text { female }\end{array}$ & 37.1 & 26.0 & 3.1 & 3.1 & & & & \\
\hline $\begin{array}{l}\text { Ajmani } \\
(1990)\end{array}$ & Nigerian & Cadavers & $\begin{array}{l}28 \text { males } \\
12 \text { females }\end{array}$ & $22.3 \pm 7.4$ & $17.3 \pm 6.6$ & $8.4 \pm 4.3$ & $7.5 \pm 4.3$ & & & & \\
\hline $\begin{array}{c}\text { Eckel et al. } \\
(1994)\end{array}$ & German & Cadavers & $\begin{array}{c}28 \text { male } \\
25 \text { female }\end{array}$ & $18.5 \pm 2.5$ & $15.8 \pm 1.1$ & $6.9 \pm 1.4$ & $6.2 \pm 1.1$ & & & & \\
\hline $\begin{array}{c}\text { Tayama } \\
(2001)\end{array}$ & Japanese & Cadavers & $\begin{array}{c}6 \text { male } \\
3 \text { female }\end{array}$ & $19.2 \pm 0.8$ & 13.6 & $7.1 \pm 1.0$ & $5.4 \pm 0.01$ & $10.2 \pm 2.7$ & 8.1 & 36.5 & 26.8 \\
\hline $\begin{array}{c}\text { Jain \& Dhall } \\
(2008)\end{array}$ & Asian Indian & Cadavers & $\begin{array}{c}20 \text { male } \\
20 \text { female }\end{array}$ & $16.4 \pm 2.7$ & $13.4 \pm 3.2$ & $6.0 \pm 0.1$ & $5.6 \pm 0.1$ & & & & \\
\hline $\begin{array}{l}\text { Gugatschka et } \\
\text { al. } \\
(2009)\end{array}$ & Austrian & $\begin{array}{l}\text { Living } \\
\text { Ultrasound }\end{array}$ & 64 male & $19 \pm 1.9$ & & $9.3 \pm 1.8$ & & $10 \pm 2.7$ & & 38.3 & \\
\hline $\begin{array}{l}\text { Hammer et al. } \\
(2010) \\
\text { Windisch et al. } \\
\quad(2010)\end{array}$ & Austrian & Cadavers & $\begin{array}{l}25 \text { male } \\
25 \text { female }\end{array}$ & & & & & $\begin{array}{l}9.2 \pm 2.5 \\
(1.5-7.8)\end{array}$ & $\begin{array}{c}6.6 \pm 1.8 \\
(2.8-16.2)\end{array}$ & & \\
\hline
\end{tabular}


Determination of resting vocal fold measurements is often difficult, complicated and/or time consuming (Perlman et al., 1984). There is also the added problem of defining the exact limits of the vocal folds (Colton, 1988). A wide range of methods and techniques have been developed for determining vocal fold dimensions. Nishizawa et al. (1988) and Schade et al. (2002) measured vocal fold length using an endoscope, whilst Su et al. (2002) measured vocal fold length during direct laryngoscopy under general anaesthetic. Since vocal fold length cannot be measured directly from radiographs, Roers et al. (2007) measured the distance from the anterior extremity of the thyroid cartilage to the spine and used this as an estimate of vocal fold length. Other authors have attempted to measure vocal fold length using CAT (Hertegård, et al., 1993) or MRI scans (Fitch \& Giedd, 1999), whilst Schuster et al. (2005) and Larsson and Hertegård (2008) used a laser projection system to measure the length and width of singers' vocal folds.

Despite differences in measurement techniques and reference points, all authors found that vocal fold dimensions differ not only between genders, with longer folds in adult males, but also between voice categories, with longer vocal folds in basses compared to tenors, and altos compared to sopranos (Pfau, 1973; Nishizawa, et al., 1988; Hertegård, et al., 1993; Eckel, et al., 1994; Benninghoff \& Drenckhahn, 2002; Su, et al., 2002; Roers, 2005; Schuster, et al., 2005; Roers, et al., 2007; Larsson \& Hertegård, 2008; Shewell, 2009). In general, vocal fold length in males is between 15 and $20 \mathrm{~mm}$ and in females between 9 and $13 \mathrm{~mm}$. This is a broad generalisation and vocal fold length in either sex can fall outside these ranges. 


\subsubsection{Larynx: Internal anatomy}

According to Hirano (1974, 1975, 1977; Hirano et al., 1981; Hirano \& Sato, 1993) the vocal folds consist of three layers of tissue (Figure 2.17): 1) an outer layer comprising epithelium and the superficial part of the lamina propria (the cover); 2) a transition layer consisting of the intermediate and deep layers of the lamina propria (layers in motion) collectively known as the vocal ligament; and 3) vocalis bundle of the thyroarytenoid muscle (the body). As discussed in section 2.1.3, deeper layers of mucosa are stiffer and are more resistant to stretching than shallower layers.

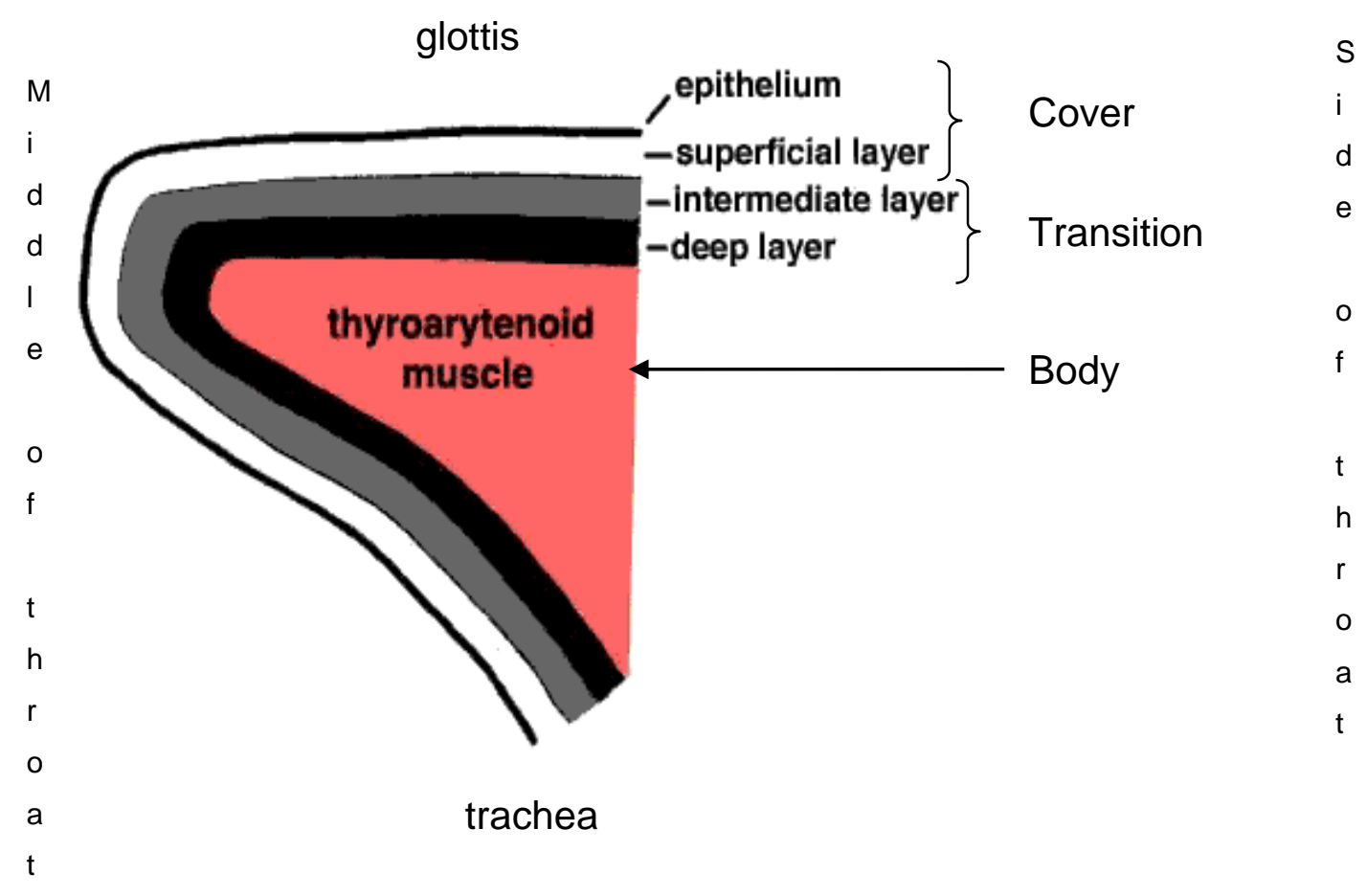

Figure 2.17: Cross section of a vocal fold, showing cover, transition and body (Michael, 2012; used with permission) 
The vocal folds open in a V-shape (Figure 2.18), with the apex of the V positioned anteriorly. The anterior ends of the vocal folds are attached to the inside of the thyroid notch, while the posterior ends (the wide part of the $\mathrm{V}$ ) are attached to the arytenoid cartilages. The folds are open during breathing and closed during phonation and singing; their movement is dictated by movements of the arytenoid cartilages.

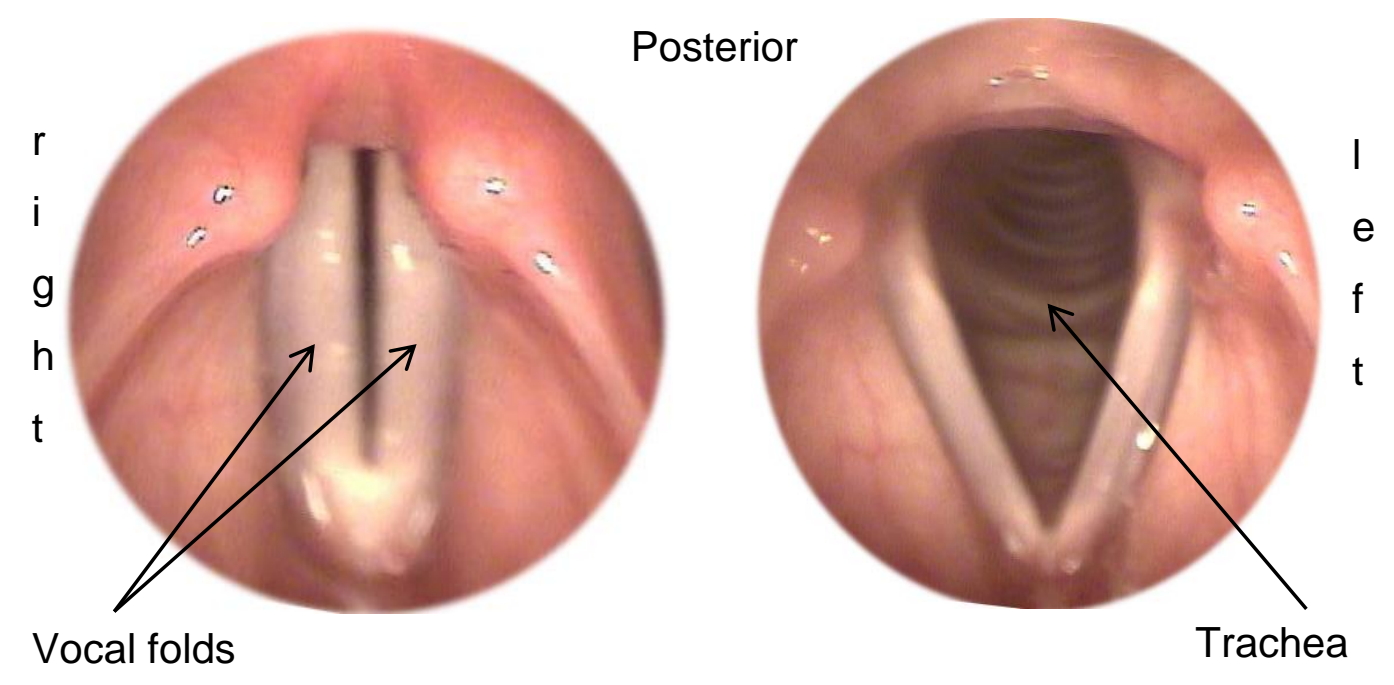

Anterior

Figure 2.18: In the left image the vocal folds are adducted, whilst in the right-hand image they are abducted (Michael, 2012; used with permission)

\section{- Glottis}

The glottis is the space between the two vocal folds. If the folds are adducted the glottis is closed and if the folds are abducted the glottis is open. Clearly, the folds must be open to breathe but air is forced through the closed glottis when speaking or singing. 


\subsubsection{Muscles of the Larynx}

There are two groups of laryngeal muscles, extrinsic and intrinsic.

Extrinsic laryngeal muscles are those that connect the laryngeal cartilages to other structures in the neck. There are four such pairs of extrinsic laryngeal muscles attached to the thyroid and cricoid cartilages from elsewhere in the chest and neck. They are connected with moving the whole larynx (Messing, 2012).

Intrinsic laryngeal muscles are those that connect the laryngeal cartilages to one another (Figure 2.19).
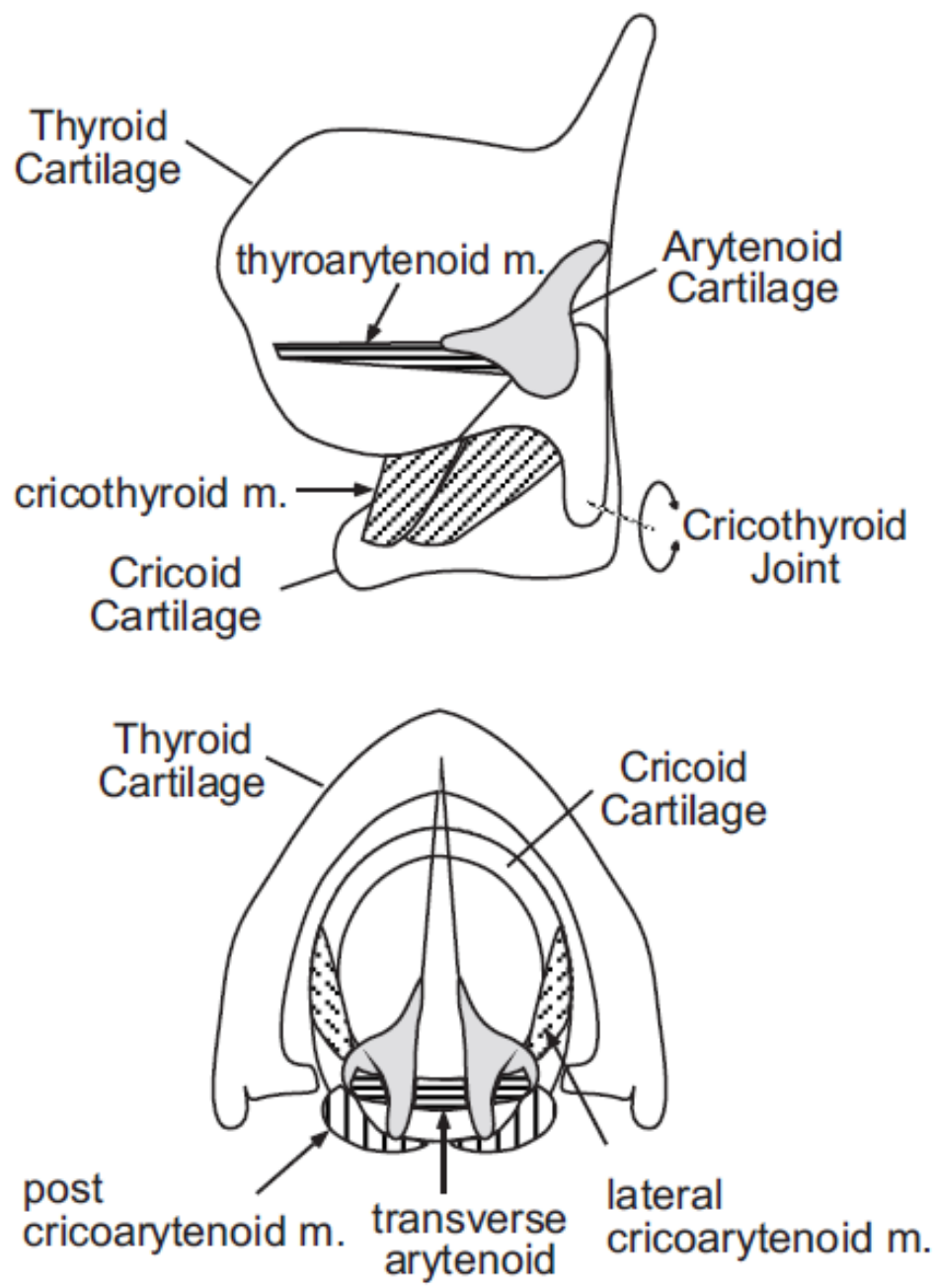

Figure 2.19: Lateral and superior view of the laryngeal intrinsic muscles (Honda, 2004) 
- Intrinsic laryngeal muscles

The intrinsic laryngeal muscles are all paired (a right and a left muscle). They are entirely responsible for changing the length, tension, shape and spatial position of the vocal folds. This is achieved by altering the orientation of the muscular and vocal processes of the arytenoid cartilages (Rosen \& Simpson, 2008). When the intrinsic muscles of the larynx contract, they pull on the arytenoid cartilages which causes them to pivot and/or glide. For example, contraction of the posterior cricoarytenoid muscles causes the vocal cords to move apart (abduction). Contraction of the lateral cricoarytenoid muscles causes the vocal cords to move closer together (adduction) (Tortora \& Derrickson, 2006). The intrinsic muscles of the larynx that regulate the length of the vocal folds are the thyroarytenoid and cricothyroid. In simple terms, the thyroarytenoid muscles shorten (and relax) whilst the cricothyroid muscles elongate (and place tension on) the vocal folds (Klimek et al., 2005).

There are several major vocal fold adductors (lateral cricoarytenoid, thyroarytenoid and interarytenoid muscles), one abductor (posterior cricoarytenoid) and two tensor muscles (cricothyroid and vocalis). Each of these muscles are discussed briefly (Figure 2.20). 


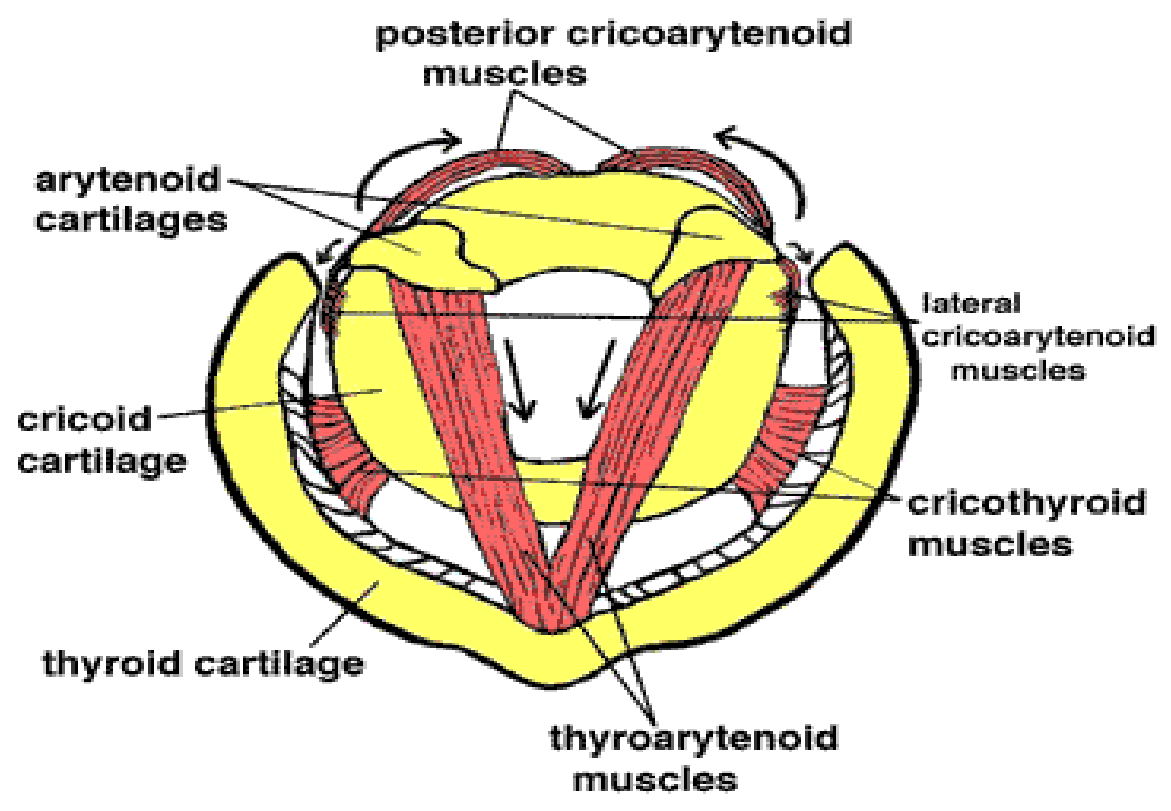

Figure 2.20: Intrinsic Laryngeal Muscles (Michael, 2012; used with permission)

- Lateral cricoarytenoid muscles

The lateral cricoarytenoid muscles are adductor muscles which close the glottis by rotating the arytenoid cartilages, bringing their vocal processes closer together.

- Thyroarytenoid muscles

The thyroarytenoid (TA) muscles lie parallel to and beside the vocal folds. They are complex paired muscles divided into two parts: Thyroarytenoid (external thyroarytenoid) and vocalis (internal thyroarytenoid). The vocalis muscle forms the medial portion of the thyroarytenoid and is adhered to the vocal ligament. Their purpose is to pull the arytenoid cartilage towards the thyroid cartilage, thereby shortening the vocal folds. This enables the vocal folds to vibrate more slowly therefore lowering the pitch. Appleman (1967) stated that the vocalis controls "... the conformation of the vocal fold in its various states of thickness and thinness during changes in pitches" (p. 46). 
- Interarytenoid muscles

There are two sets of the interarytenoid muscles: the transverse and the oblique arytenoids. They bring the two arytenoid cartilages together, thus adducting the vocal folds.

- Cricoarytenoid muscles

The posterior cricoarytenoid muscles are the abductor muscles which open the glottis by pulling the posterior ends of the arytenoid cartilages apart and rotating their vocal processes to allow the vocal cords to abduct. To some extent, these are the most important muscles involved in vocal production since they are the only muscles that cause the vocal cords to move apart.

- Cricothyroid muscles

The cricothyroid (CT) muscles are the largest of the intrinsic muscles. When the CT muscles contract, they lengthen the vocal folds by pulling the thyroid cartilage down and forward on its hinge with the cricoid cartilage. This increases the distance between the arytenoids and the thyroid notch. As the vocal folds lengthen, they become thinner (decrease of vocal fold vibratory mass) and taut (increased vocal fold tension). This causes them to vibrate faster as air is passed through them and therefore raises the pitch (Hirano, 1981; Strong \& Vaughan, 1981; Shipp, 1982; Honda, 2004; Michael, 2012). 


\subsubsection{Cricothyroid joint}

The cricothyroid joint (CTJ) is the articulation between the inferior cornu of the thyroid cartilage and the side of the cricoid cartilage (Figure 2.21). The primary movement at these paired synovial joints is rotation around a transverse axis but some gliding movements in an anteroposterior (horizontal) and craniocaudal (vertical) direction can also occur (Maue \& Dickson, 1971; Sataloff et al., 1998). The CTJ plays a key role in adjusting pitch. When the cricothyroid muscles contract, the thyroid and cricoid cartilages are approximated anteriorly, narrowing the CT space and elongating the vocal folds which results in an increase of pitch with a consecutive stretching and increasing of tension (Hammer, et al., 2010).

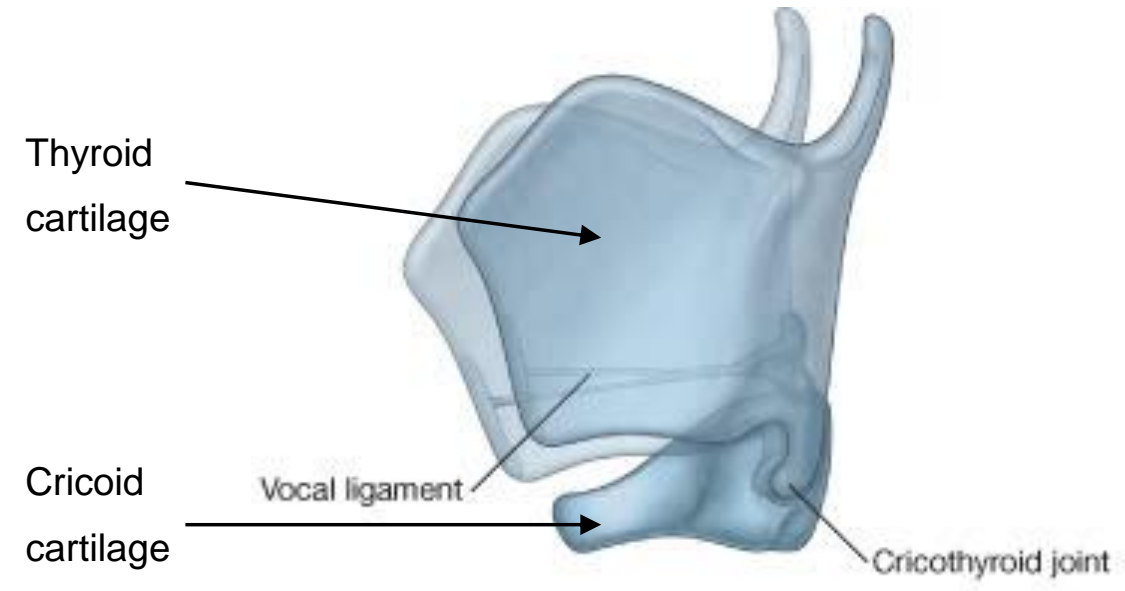

Figure 2.21: Cricothyroid Joint (Drake et al., 2005; used with permission)

The CTJ has a highly variable anatomy, and this determines movement capabilities (rotation and anteroposterior gliding) in the CTJ and direct determination of vocal fold lengthening. However, there have been suggestions that these movement capabilities of the CTJ are limited by its anatomical structure (Mayet \& Mundnich, 1958). Articular joint surface, joint type, effective rotational axis, capsule configuration and ligaments surrounding the joint all determine its mobility. Studies have proven that there is a clear correlation between CTJ anatomy and vocal fold lengthening. (See Appendix D for full explanation). Restrictions on rotational ability depend on where the effective rotational axis lies. A low 
rotational axis permits more rotation of the CTJ thus more vocal fold lengthening can occur. Conversely, less vocal fold lengthening occurs with a higher rotational axis. Restrictions in gliding would be caused by a welldefined articular joint surface with a tight capsule and ligaments (Mayet \& Mundnich, 1958; Kahane, 1994; Hammer, et al., 2010). In contrast, a poorly developed articular joint surface with a loose capsule and slack ligamentous cartilages would increase the potential for gliding (Maue \& Dickson, 1971; Vilkman et al., 1987). Thus gliding and pars obliqua contraction may serve as a function of individual anatomical variations of the CTJ (McHenry et al., 1997). 


\subsubsection{Muscular control of fundamental frequency}

The cricothyroid (CT) muscle plays a key role in adjusting $F_{0}$, especially in raising pitch. Cinematographic and radiographic observations (Ranke \& Lullies, 1953; Hollien, 1960; Fink, 1962), as well as electromyography (EMG) studies (Arnold, 1961; Yanagihara \& von Leden, 1968; Hirano et al., 1969; Gay et al., 1972; Atkinson, 1978; Shipp et al., 1979; Roubeau et al., 1997; Kochis-Jennings et al., 2012), have all demonstrated that the CT muscle is the prime contributor to controlling $F_{0}$ during phonation (Freedman, 1956; Hast, 1966; Yanagihara \& von Leden, 1968; Haglund, 1973; Stone \& Nuttall, 1974; Braund et al., 1988; Pešák, 2009). Further compounding the complexity of $F_{0}$ control is the intrinsic complexity of the CT muscle itself. Traditionally, the human CT muscle has been described as consisting of straight (pars recta) and oblique (pars obliqua) parts. A third deep part of the muscle (horizontal part) has been identified in humans (Mu \& Sanders, 2007). Each part has distinct EMG activity but only the first two parts have been studied in detail (Hiroto et al., 1967; Honda, 1988; McHenry, et al., 1997; Hong, et al., 1998; Hong et al., 2001). Most studies agree that the pars recta and pars obliqua have different functions across $F_{0}$ : the pars recta showed almost constant activity during phonation regardless of $F_{0}$ variation, whereas the pars obliqua had a linear relationship with $\mathrm{F}_{0}$, demonstrating greater activity with a higher $\mathrm{F}_{0}$ (Honda, 1983, 1988; Hong, et al., 1998; Hong, et al., 2001; Honda, 2004). Even though stimulation of the pars recta resulted in greater increases in $F_{0}$ than in the pars obliqua, the combined activity of both parts was associated with the greatest increase in $F_{0}$. This implies that the synergistic activities of both parts are important in adjustment of vocal fold length.

The cricothyroid joint (CTJ) is the main framework for $\mathrm{F}_{0}$ control of the human voice (Honda, 2004). It allows an external elongation of the vocal fold performed by the CT muscle with a consecutive stretching and increasing of tension (Hammer, et al., 2010). The pars recta and pars obliqua each have a different direct action on the CT joint (Honda, 2004). The main movement of the CTJ is rotation (Fink \& Demarest, 1978). This 
is initiated by the contraction of the pars recta, which rotates the thyroid cartilage down towards the cricoid cartilage along a vertical axis (Vilkman et al., 1996), reducing the CT space (Figure 2.22). According to Vilkman (1997), this is the most important factor affecting longitudinal tension of the vocal folds, functioning over the entire $F_{0}$, resulting in a simultaneous raising of the $\mathrm{F}_{0}$ (Hong, et al., 1998).

In contrast, the pars obliqua moves the cricoid cartilage backwards (forward translation of the thyroid cartilage) with simultaneous forward anteroposterior gliding at the CT joint (Freedman, 1956; Vilkman, 1987; Vilkman, et al., 1987) (Figure 2.22). It causes longitudinal vocal fold lengthening which is important for $\mathrm{F}_{0}$ regulation in extremes of pitch range particularly at the upper end (Sonesson, 1982; Honda, 2004). As this is mainly a forward horizontal movement, there is minimal CT space movement. Several investigators have determined it corresponds to less than $50 \%$ of changes in vocal fold length (Sonninen, 1956; Sonesson, 1959; Fink \& Demarest, 1978) but since joint translation takes place parallel to the longitudinal axis of the vocal fold, it can stretch the vocal folds more effectively than rotation. Therefore, this secondary movement is also important in controlling $F_{0}$, especially for higher frequencies. Both rotational and horizontal gliding movements combined cause a significantly greater elongation of the vocal folds as compared to just using rotation (Hammer, et al., 2010). 


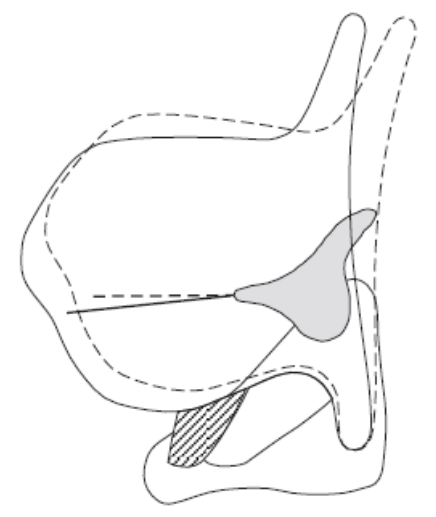

(a) Rotation

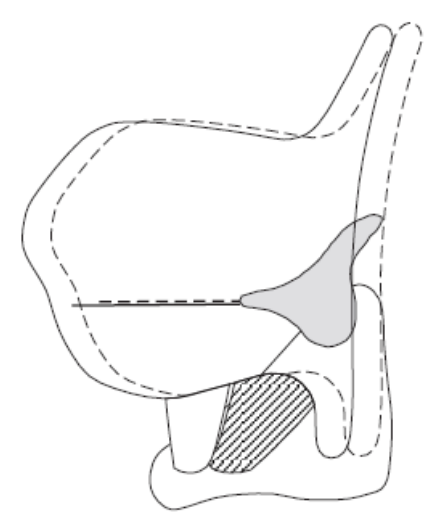

(b) Translation

Figure 2.22: Rotation and translation of the cricothyroid joint respectively (Honda, 2004)

The thyroarytenoid (TA) muscles are also considered important intrinsic laryngeal muscles in $F_{0}$ regulation. The TA causes vocal fold shortening contributed to by CTJ rotation (Honda, 2004) and widens the CT space. Contraction of the TA does not only act on the CT joint, but also changes the property of the deep layer of the vocal fold (Honda, 2004). Generally, an increase in TA activity increases the thickness of the vocal folds, so a decrease in the activity of the vocalis muscle occurs with a shift from a heavier register to a lighter one.

Functionally however, the TA role in $F_{0}$ control is not straightforward. It interacts with the CT in raising or lowering $F_{0}$ (Shipp \& McGlone, 1971; Shipp \& Morrissey, 1977; Titze et al., 1989; Lindestad et al., 1991). Many EMG observations of the TA have indicated that its activity increases $F_{0}$ despite its potential Fo lowering effect (Arnold, 1961; Hirano, et al., 1969; Gay, et al., 1972; Atkinson, 1978; Shipp, et al., 1979). The complex balance of CT and TA muscle activity (Larson et al., 1987) appears to be dependent on different parts of the range (Titze, 1993). TA activity correlates with a rise in $\mathrm{F}_{0}$ as long as the CT activity is not at its maximum, i.e. at lower fundamental frequencies and lower vocal intensities (Titze, 2000; National Center for Voice and Speech, 2013a). Conversely, an 
increase in TA activity tends to lower $F_{0}$ at higher fundamental frequencies and low vocal intensity (especially in falsetto voice) (Titze, et al., 1989).

The CT muscle acts as an antagonist to the vocalis muscle, and therefore isometric tension can occur between these two muscles (Titze, 1981). If the CT and TA reach an isometric point, vocal fold tension increases without further lengthening of the vocal folds. Consequently, the CT and TA have opposing effects on the change of length of the vocal fold. The CT muscles lengthen the vocal folds while the TA muscles shorten the vocal folds. Therefore, at lower pitches the vocalis muscle is used more to shorten and thicken the vocal folds. In the middle range there is a balance of TA and CT activity, whilst in the high range the activity of CT dominates and vocalis must relax (Miller, 1986b). Thus, to produce high frequencies the vocal folds must be long, thin and taut, conversely producing low frequencies requires short, thick and relatively slack vocal folds. Great skill is necessary to achieve transitions throughout different registers, especially from middle to head register, without a break. As the CT and vocalis are innervated by different muscles (superior laryngeal nerve and recurrent laryngeal nerve respectively) it is possible to learn to activate these adjacent muscles selectively and independently balance them to optimise the control of $F_{0}$ and vocal range (Titze, 2000).

If CT muscle activity has reached its physical limit for contraction and stretched the collagenous fibres within the vocal folds to their limit for elongation, $F_{0}$ can only be increased further by increasing vocal fold tension and stiffness alone. However according to Miller (2000) this should be avoided because "the inner elastic tissues of the vocal folds are not constructed to sustain stressful action over long periods of time without damage" (p. 28). To avoid this excessive stress on the vocal folds, it has been proposed that damping should be used instead (Titze \& Hunter, 2004). 


\subsection{Cricothyroid space}

The cricothyroid (CT) space is the interval (gap) between the anterior inferior border of the thyroid cartilage and the anterior superior border of the cricoid cartilage (Goumas et al., 1997) (Figure 2.23). It is bridged by fibrous tissue that forms the cricothyroid membrane. The cricothyroid ligament is the thickened vertical middle segment of the cricothyroid membrane. The band is narrower at the top where it is attached to the thyroid cartilage, and broadens as it descends to attach to the cricoid cartilage (Reidenbach, 1996).

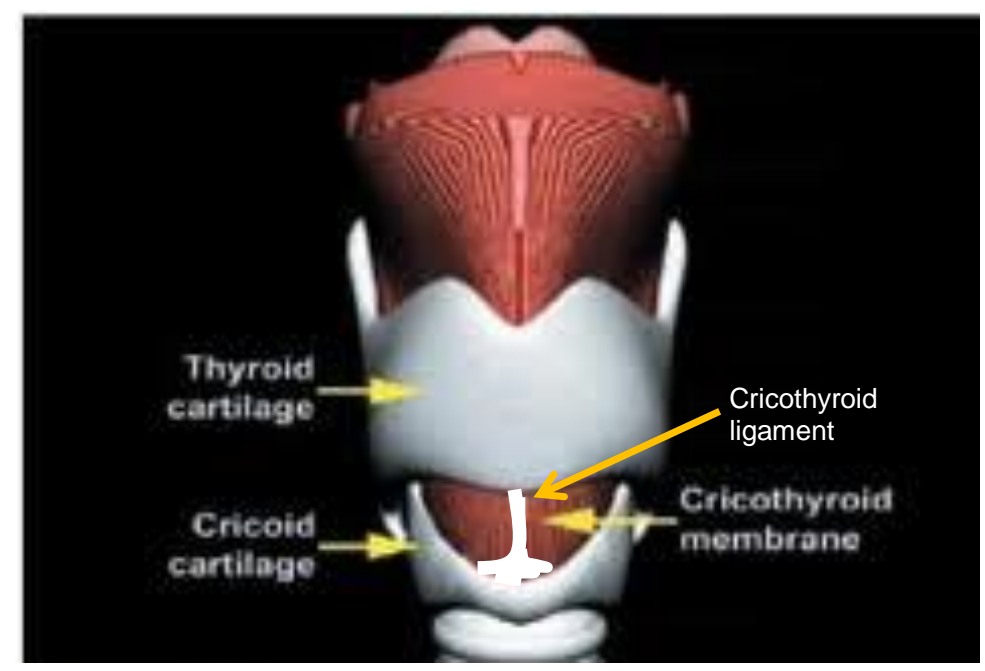

Figure 2.23: Cricothyroid Space (Hsiao \& Pacheco-Fowler, 2008; used with permission)

Clinically, the CT space and its relation to other intralaryngeal structures is important in the surgical modification of vocal pitch. The two most common operations for achieving this are Cricothyroid Approximation (CTA) and Anterior Commissure Laryngoplasty. (See Appendix A for an explanation of these surgeries). Cadaver studies have been performed to define the best surgical approach to minimize tissue damage (Reidenbach, 1996) and to study the anatomy of the CT space with specific reference to structures within $1 \mathrm{~cm}$ of the midline (Goumas, et al., 1997). 


\subsubsection{Vocal pitch and the cricothyroid space}

The region of the cricothyroid space has been referred to as the cricothyroid visor by some authors (Ardran \& Kemp, 1966; Harris, et al., 1998) because the cricoid and thyroid cartilages articulate in a similar manner to a metal helmet in a suit of armour. When the thyroid cartilage tilts forward on the cricoid cartilage, the cricothyroid visor closes and the vocal folds lengthen, producing a higher pitch. Conversely, the cricothyroid visor opens wider when singing at a low pitch (Harris, et al., 1998). As Ardran and Kemp (1966) stated, "...there is no doubt that closing of the cricothyroid space elongates and tenses the vocal folds and is associated with the production of high pitched sounds" (p. 653). It is important that the visor can be opened and closed quickly and freely to enable adjustments in the length and tension of the vocal cords to accommodate the wide range of pitches required for singing. If the CT membrane within the visor becomes too tight then problems with phonation can occur (Shewell, 2009).

The early 1900s saw several studies investigate the relationship between $F_{0}$ and the CT space. Using radiography, it was shown that the visor is in a partly open neutral position during quiet respiration (Sonninen, 1956; Fink \& Demarest , 1978) and the CT space became narrower with rising pitch (Moeller \& Fisher, 1904) and wider with decreasing pitch (Vilkman et al., 1997). These findings were subsequently confirmed by others (Arnold, 1961; Ardran \& Kemp, 1966; Harris, et al., 1998). However, Laukkanen et al. (2002) demonstrated that the decrease in CT space was not linearly related to rising pitch. Smaller decreases occur in the mid-range pitches, (and there are dips on either side at passaggio points) and, at the very highest pitches, the decrease in CT space is extremely small. Furthermore, trained singers have smaller changes in the CT space in the mid-range pitches compared to non-singers. This finding was confirmed by Gugatschka et al. (2009). These results parallel data obtained by Sonninen (1954) and Sonninen et al. (1999) who showed that the pitch 
related increase in vocal fold length was nonlinear, with smaller increments at higher pitches. Vilkman et al. (1997) discovered that when the CT space was narrowest, $\mathrm{F}_{0}$ continued to rise and that vocal pitch could decrease further after the CT space was maximally open.

\subsubsection{Cadaver studies on the cricothyroid space}

The majority of studies to investigate the anatomy and function of the CT space have been carried out in human cadavers (Vilkman, et al., 1987; Reidenbach, 1996; Goumas, et al., 1997). This has been accepted as a legitimate way to study human larynges (Van Den Berg \& Tan, 1959) but it should be recognised that whilst the studies produce useful results, living anatomy may be slightly different.

Kitajima et al. (1979) designed an experiment using excised human larynges to establish the relationship between the anterior CT space and vocal pitch. They showed that stimulation of the CT muscle decreased the CT space and raised the vocal pitch. In addition, the relationship between CT space and vocal pitch, expressed in semitones, was almost linear. Another study by Hammer et al. (2010) was conducted to determine the correlation between anterior CT space distance and vocal fold length (Figure 2.24). They noted that males had significantly greater maximum and minimum CT spaces than females when the CT space was opened and closed by manual rotation of the CT joint. Despite this, changes in the dimension of the CT space caused nearly the same changes in length and tension of the vocal folds in men and women. This was also previously noted by Filho et al. (2005) who attributed it to males having a more posterior cricothyroid articulation on the cricoid ring and longer vocal folds such that overall vocal fold elongation was similar to that of females. 


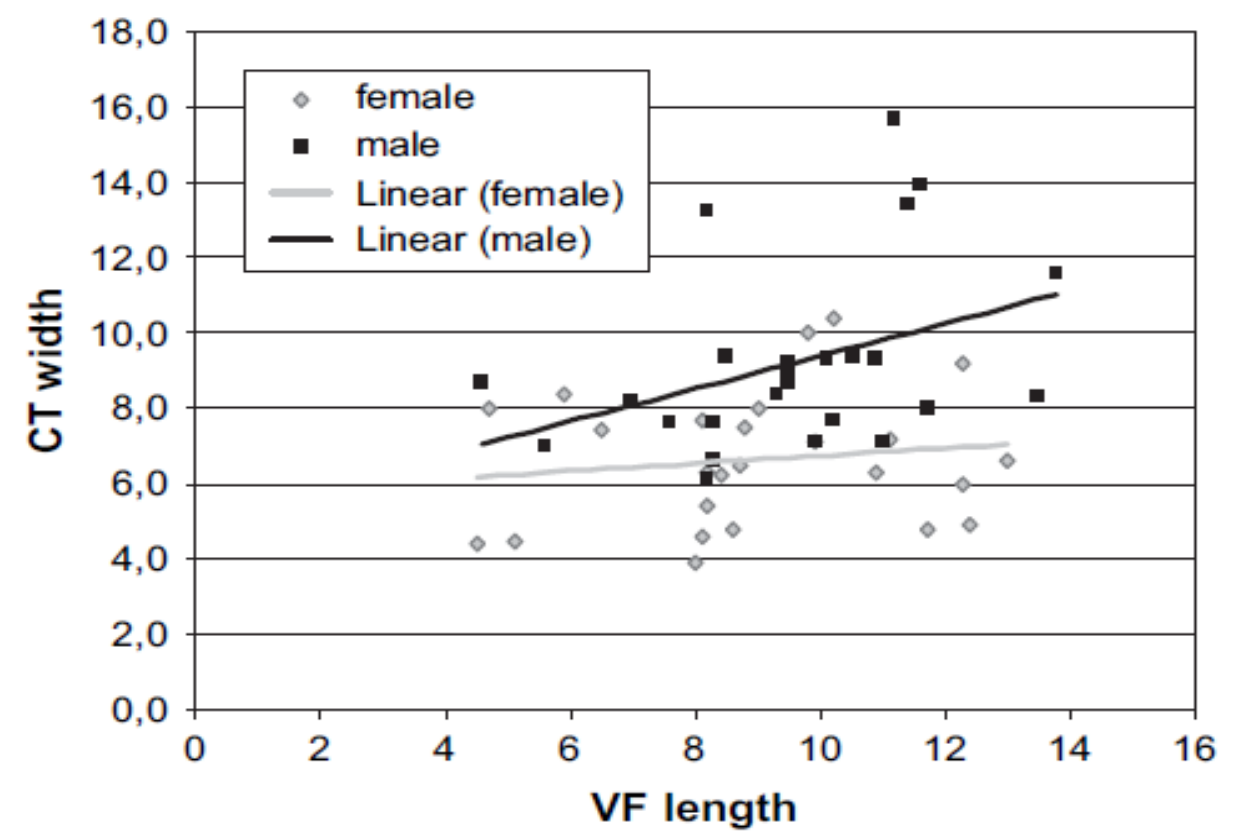

Figure 2.24: Correlation between the width of the CT space and the length of the vocal folds (Hammer, et al., 2010; used with permission from Elsevier)

\subsubsection{Ultrasound studies of the cricothyroid space}

There are many techniques for measuring the CT space. The space can be directly visualised by fiberoptic endoscopy but this is invasive and subject to measurement problems (Vilkman, et al., 1997). Video fluorography is another method, but the cricoid and thyroid cartilages are often poorly visualised which could lead to measurement problems, and, like straight $\mathrm{X}$-rays and CAT scans, there are radiation risks (Vilkman, et al., 1997). Ultrasound (US) has been widely used in medicine to study all regions of the human body. In otolaryngology, US is used to visualise many structures in the neck, including cartilages, glands and lymph nodes. Furthermore, this method of imaging is painless and carries no harmful radiation risk. Since the cricoid and thyroid cartilages are readily visible, the space between them can easily be measured.

Most of the US studies carried out on the CT space have focused on pitchsynchronous changes in the anterior CT space. They have shown that the CT space decreases with rising pitch and increases with lowering pitch. This confirms that changes in the anterior CT space reflect changes in $\mathrm{F}_{0}$. 
Laukkanen et al. (2002) used ultrasound to show that when singers sang rising intervals of perfect fifths throughout their range, the CT space decreased. Gugatschka et al. (2009) confirmed these findings. Vilkman et al., (1997) used ultrasound to measure the CT space in participants speaking a three and a five word sentence. They demonstrated that the CT space widened with decreasing pitch as a function of speech declination. The results obtained in these studies show that ultrasound imaging is a reliable method for measuring the anterior CT space.

A few difficulties measuring the CT space with US were encountered by Vilkman et al. (1997) and Laukkanen et al. (2002). Reference points for measurement were the calcified areas close to the anteroinferior edge of the thyroid cartilage and the anterosuperior edge of the cricoid cartilage. Vilkman et al. (1997) noted that these landmarks may be affected by soft tissue displacement. Laukkanen et al. (2002) noted that these points did not necessarily correspond to the exact border of the cartilages and so the measured CT distance was not always accurate. They also found that the vertical position of the larynx changed over a pitch range, which affected the measuring points and contours of the calcified landmarks. For these reasons only changes in CT distances could be measured and values were comparable over only a limited pitch range.

In both studies, the US transducer was placed against the skin overlying the anterior aspect of the larynx in the midline. To obtain clear images of the thyroid and cricoid cartilages and to avoid projection errors, contact between the skin and the US probe had to be very tight. Vilkman et al. (1997) found that a silicone stand-off pad had to be used to improve contact and image quality in complicated cases. Laukkanen et al. (2002) found that pressure on the larynx from the US probe prevented anteroposterior movement of the larynx and affected projection of the image. Furthermore, participants were laying supine, which could affect voice production and interfere with voice control habits. Both the supine position and the pressure of the US probe on the neck may impair the 
function of the muscles that assist anteroposterior gliding at the CTJ. Therefore, participants may have been forced to use rotation at the CTJ more than usual when raising the pitch. Female subjects, in particular, may have adopted a more falsetto-like singing style requiring less elongation of the vocal folds.

Vilkman et al. (1997) found that the limited frame rate of the video recorder caused some blurring of the images during rapid changes and the timing of the measurements was hampered by differential framing of video and audio signals. They commented on the different strengths and weaknesses of manual measurements and those made with a computer cursor but found that both methods correlated well and that reasonably reliable results could be obtained using either.

In summary, even though limitations are recognised in studies using US to measure anterior CT distance, US imaging is nevertheless reliable.

- Ultrasound Imaging

With ultrasound, cartilaginous structures such as the thyroid cartilage, cricoid cartilage and tracheal rings are homogeneously hypoechoic (i.e. they reflect relatively few ultrasound waves and appear dark in sonographic images), and their intraluminal surfaces are outlined by a bright air-mucosa interface. They can be seen clearly as shown in Figure 2.21. Both the thyroid and cricoid cartilages may demonstrate a variable degree of central internal echogenicity related to increased tissue density.

On sagittal view, the thyroid cartilage was visible as a linear hypoechoic structure highlighted by the bright air-mucosa interface at its posterior surface. The cricoid cartilage on sagittal view had a rounded hypoechoic appearance (Singh et al., 2010).

Sonographically, the cricothyroid membrane can be demonstrated on the sagittal view as a hyperechoic band linking the hypoechoic thyroid and 
cricoid cartilages (Figure 2.25); hyperechoic (also sometimes called echogenic) structures have many internal echoes and appear bright in sonographic images. 

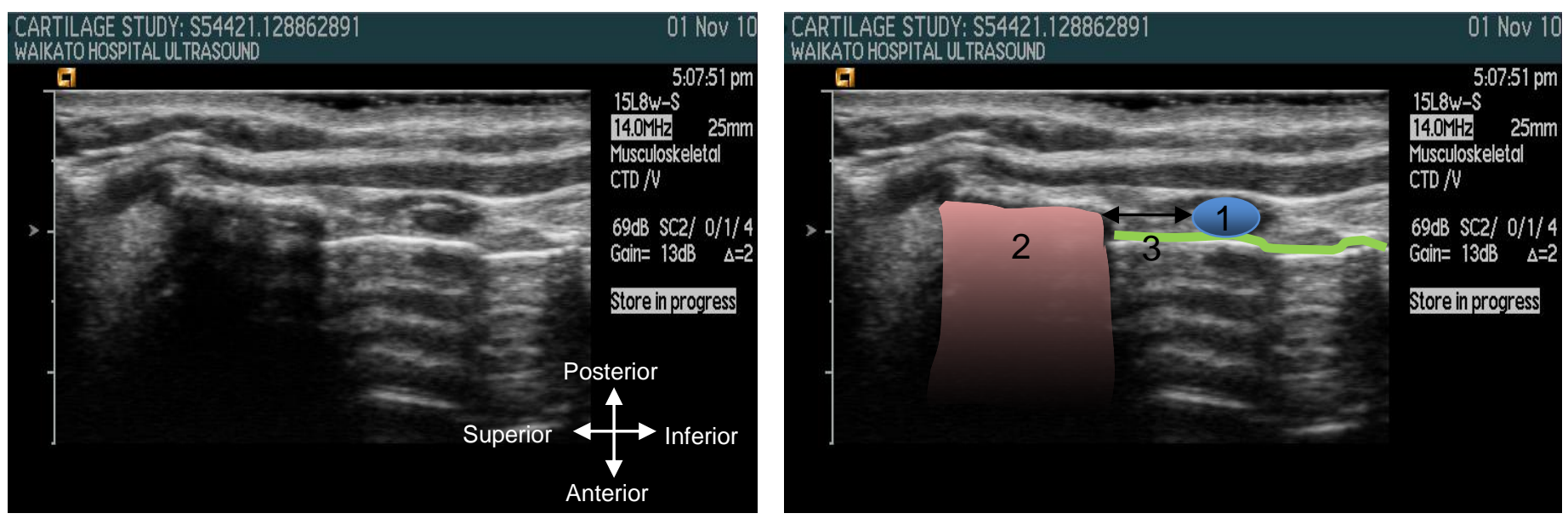

Figure 2.25: Ultrasound image showing cartilaginous structures of the larynx on sagittal view. The right hand image is a coloured version of the left to highlight anatomical structures. 1. Cricoid cartilage 2. Lower edge thyroid cartilage 3. Tracheal lumen (green line). Arrow: Anterior cricothyroid space. There is a centimetre scale bar on the left of each image (Source: Author) 


\section{Rationale for study}

There is growing evidence that the CT space is important in determining vocal pitch (Titze, 1994; Tayama, et al., 2001). The CT space decreases when singing higher pitches. Cricothyroid approximation surgery is used to elevate the speaking voice of transsexuals by reducing the CT space (Isshiki, et al., 1974; Isshiki, 1981; Isshiki et al., 1983; Isshiki, 1989; Neumann, et al., 2002; Yang, et al., 2002; Lawrence, 2004). Further, in excised larynges from cadavers, a nearly linear relationship exists between vocal pitch and CT distance (Kitajima, et al., 1979).

It is clear from the literature review (section 2.6.3) that studies of the CT space have focused mainly on pitch synchronous changes, but have not explored the relationship with overall vocal range. It has been shown by Laukkanen (2002) and Gugatschka (2009) that the CT space dimensions change with $F_{0}$. However, the suggestion by Titze (1998) that a large CT space is important for a wide vocal range appears not to have been systematically investigated.

Studies have shown that ultrasonography is a reliable technique for measuring the CT space, although most of these studies have been performed on only small numbers or untrained singers (Vilkman, et al., 1997; Laukkanen, et al., 2002; Gugatschka, et al., 2009). Furthermore, other possibly related morphological parameters, such as the length and height of the thyroid and cricoid cartilages, or basic physical parameters such as subject weight and height, have not been considered in these studies. It appears that no study has been performed previously on healthy, trained female singers. 


\section{Hypothesis}

A female singer's laryngeal anatomy, in particular their cricothyroid midline interval, correlates with vocal range.

\subsection{Study Aims}

The purpose of this research was twofold. The primary goal was to determine whether the dimensions of the resting cricothyroid interval correlate with vocal range in female singers. A second aim was to investigate potential associations between voice categories (soprano and mezzo-soprano), age, laryngeal dimensions (thyroid and cricoid cartilage heights), neck dimensions (circumference and length), anthropometric indices (weight, height, $\mathrm{BMI}$ ), and habitual speaking fundamental frequency (SSF). 


\section{Materials and Methods}

\subsection{Participants}

Following written informed consent, 43 healthy female participants were enrolled and underwent a systematic ultrasound examination of their larynx. Anthropometric and vocal data were also collected. Inclusion / exclusion criteria for this study were as follows:

- Classically trained female singers only aged between 18 and 65 years at a minimum vocal performance level of second year university or above.

- Voice has to be in a comfortable Fach.

- No history of voice disorder.

- No active upper respiratory tract infection or allergy, and to be symptom free on the day of testing.

\subsection{Equipment and Measurements}

In addition to age (in years) and ethnicity ${ }^{4}$ (Statistics New Zealand categories; version1.0, 2005), anthropometric, vocal and ultrasound measurements were taken.

\footnotetext{
${ }^{4}$ Ethnicity should not be confused with other related terms. Race is a biological indicator and an ascribed attribute. Ancestry is a biological and historical concept and refers to a person's blood descent. Citizenship is a legal status. These terms contrast with ethnicity which is self-perceived and a cultural concept. Ethnic origin is a person's historical relationship to an ethnic group, or a person's ancestors' affiliation to an ethnic group, whereas ethnicity is a person's present-day affiliation (Statistics New Zealand, 2013)
} 


\subsection{Anthropometric Data}

Height $(\mathrm{cm})$ was measured to within $1 \mathrm{~mm}$ using a stadiometer. It has a vertical scale and a movable headboard and is affixed on the wall at a predetermined height. The barefooted participant stood under the stadiometer (seca, Birmingham, U.K) with the back of their head and heels flat against the wall. (Figure 5.1)

Body weight was measured to the nearest $10 \mathrm{~g}$ (with light clothing on) using Oregon scientific digital scales, Model GA101 (Tualatin, Oregon USA). By entering age and height into the scales, BMI $\left(\mathrm{kg} / \mathrm{m}^{2}\right)$ and $\%$ body fat was calculated. (Figure 5.2 )

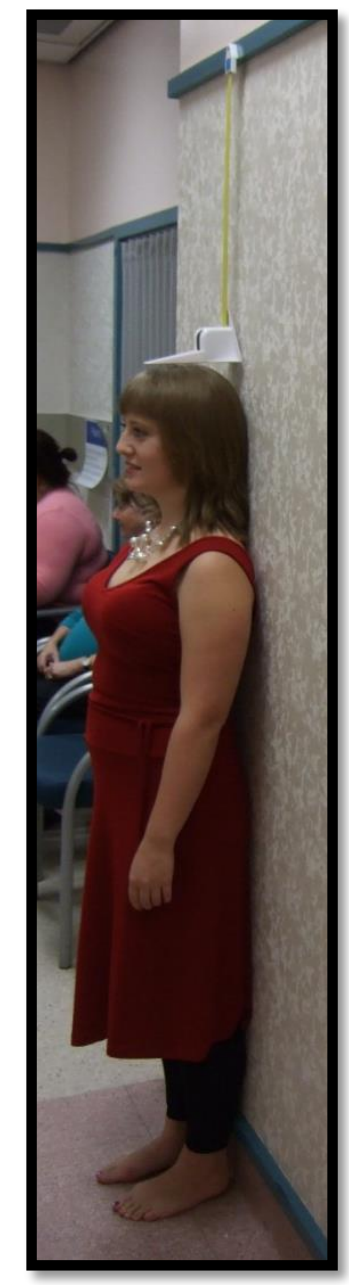

Figure 5.1: Stadiometer (Source: Author)

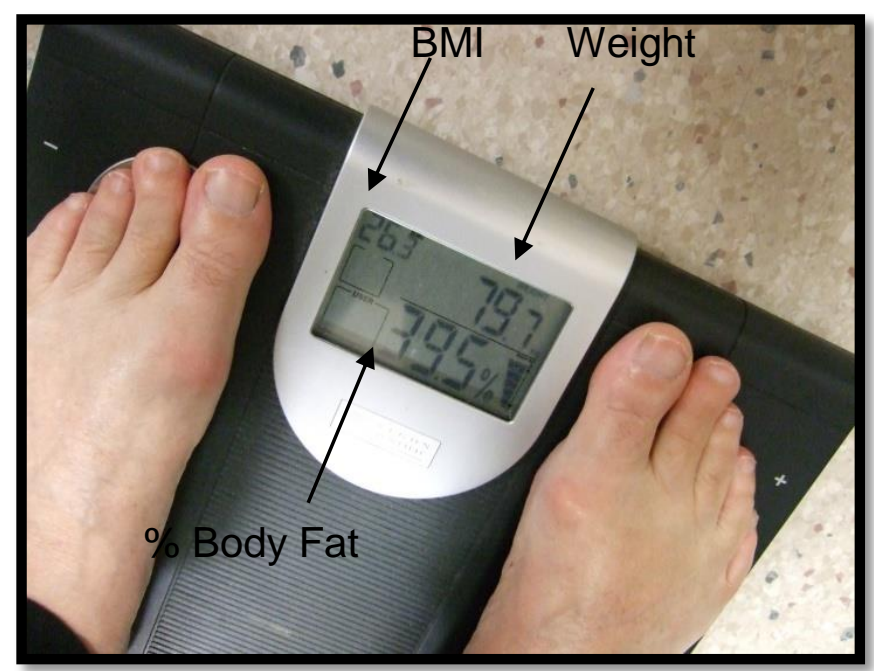

Figure 5.2: Scales showing readings obtained (Source: Author) 


\subsection{Neck Dimensions}

Neck length and circumference to the nearest $\mathrm{mm}$ were measured with participants seated upright in a chair with their face directed forward and shoulders relaxed. The average of three repeated measurements was recorded.

Neck circumference was recorded just below the larynx and perpendicular to the axis of the neck using a plastic tape measure (Ben-Noun et al., 2001) (Figure 5.3).

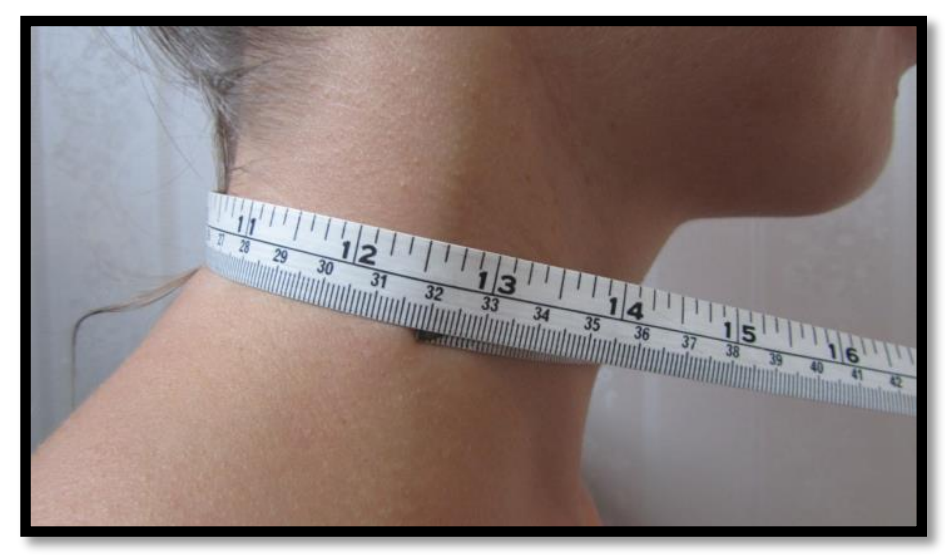

Figure 5.3: Neck circumference measurement (Source: Author)

To measure neck length using a standardised and consistent technique, anatomical landmarks were used to position the head in a neutral position (Figure 5.4). The Frankfurt Plane is an anatomically based co-ordinate which is commonly used to orientate the human skull. It is defined as a plane passing through the inferior margin of the left orbit (the left orbitale) and the upper margin of the ear canal or external auditory meatus (the porion). This equates to a neutral head position in the living subject and is almost parallel to the true horizontal (Cheng et al., 2012). 


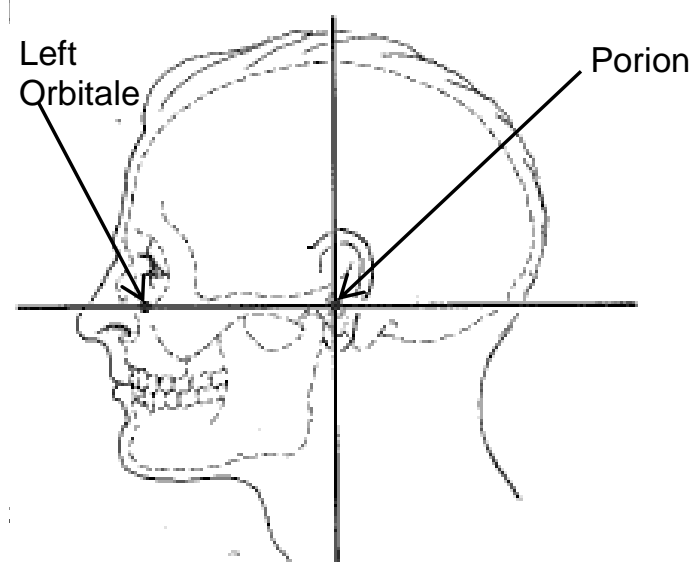

Figure 5.4: Neutral head position using Frankfurt plane (Manén, 1974; used with permission)

A homemade device (Figure 5.5) constructed out of a CD case, ruler and spirit level was used to align the head in a neutral anatomical position. The edge of the CD case was placed against the porion and the edge of the ruler against the left orbitale. The head was adjusted vertically until the spirit level showed it to be horizontal (Figure 5.6B). After this neck length was measured with a ruler from the upper surface of the suprasternal notch to the under surface of the tip of the chin (Figure 5.6C). Four sites were marked on each participant with a black felt marker pen to make these measurements: left orbitale, left porion, suprasternal notch and tip of chin (Figure 5.6A).

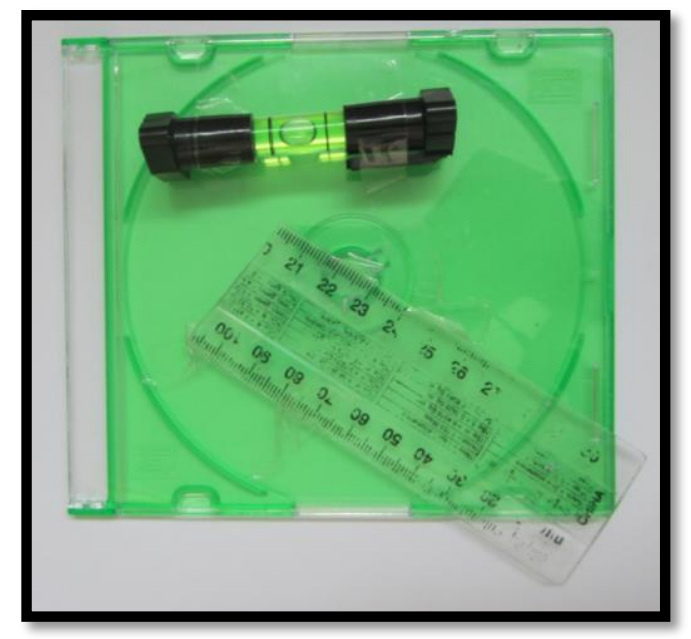

Figure 5.5: Device used for anatomical positioning of head (Source: Author) 

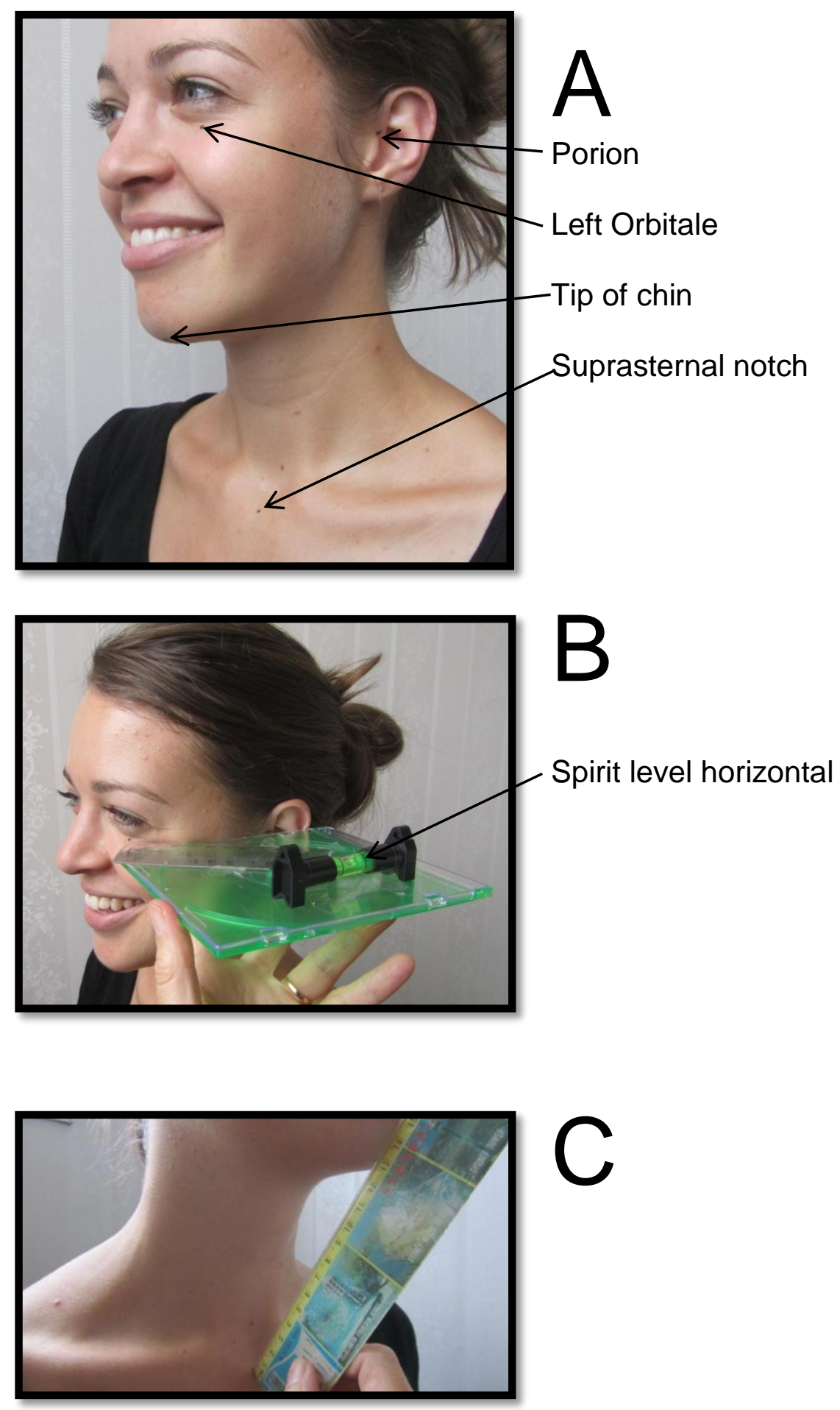

Figure 5.6: Procedure for neck length measurement.

A: Surface landmarks marked

B: Neutral head position obtained

C: Measurement of neck length

(Source: Author) 


\subsection{Vocal Data}

Vocal data included: Fach, Vocal range (practice and performance ranges) expressed in note names and semitones and fundamental frequency of speech.

Each participant was asked to state their perceived voice type using the Fach classification. As Fach types can overlap, several singers were considered as belonging in two categories. A handout with the available options and an explanation of each category was given to the participant if required (see Appendix B). Due to the large number of Fach types, only the common Fach types of female voices were used. The options given were: Coloratura soprano, Soubrette, Lyric soprano, Spinto, Dramatic soprano, Lyric mezzo-soprano, Dramatic mezzo-soprano and Contralto.

A simple definition of vocal range is the span from the lowest to the highest note that a singer can produce. However, to a classically trained singer, this definition is refined to distinguish between a 'practice range' and a 'performance range'. For the purpose of this thesis, practice vocal range is defined as the total vocal range that an individual voice can physically produce, irrespective of vocal quality. In contrast, a performance vocal range is considered to represent the lowest to highest notes that an individual would sing publicly. The quality of sound within a performance range is typically regarded as being aesthetically pleasing (Appelman, 1967).

To ascertain the two vocal ranges as defined, each participant was asked to state their practice and performance ranges according to the set criteria (above). Counting in semitones each participant's practice range and performance range was then calculated, assigning $\mathrm{C}^{4}$ as middle $\mathrm{C}$.

Fundamental frequency ( $\mathrm{F} 0$ ) of speaking voice (in $\mathrm{kHz}$ ) was recorded using VoceVista - Pro Multimedia version 1.0, 2008 voice analysis software (Visualization Software LLC, Stafford, Virginia, USA) whilst reading a passage of prose (Appendix E) at a comfortable pitch and 
loudness. Each participant was taken into a booth and asked to read three short passages into a microphone (Laser A0-MICD02) held approximately $12 \mathrm{~cm}$ from the mouth, which was connected to a laptop computer. The sound spectrogram (F3 function) of the VoceVista - Pro programme was used to analyse the readings. The frequency range was set from zero to $5 \mathrm{kHz}$ and at the end of each reading the display was "frozen" (Figure 4.7).

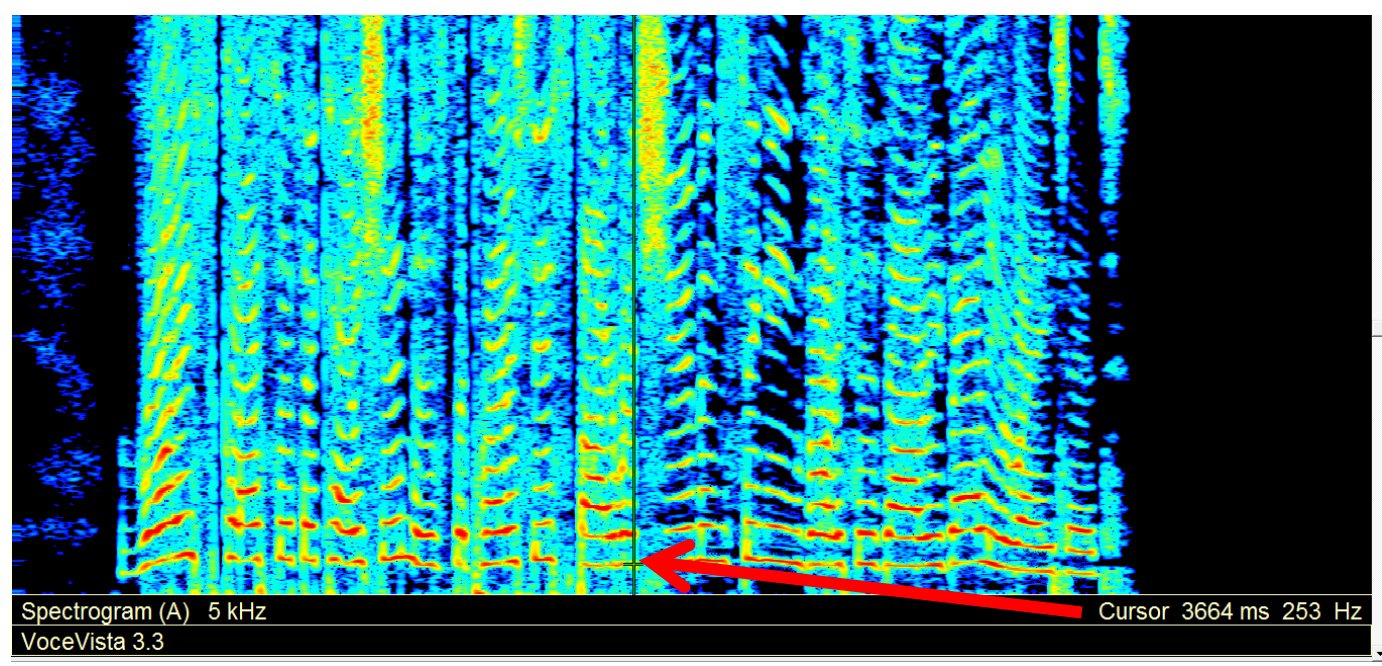

Figure 5.7: Sound spectrogram from a participants reading passage (Source: Author)

In speaking, the vocal folds continually change their length and tension so vibrate at a number of different frequencies and produce a number of different pitches (Harris, et al., 1998). As consonants break the harmonic line, each individual word is seen as a distinct harmonic band followed by a slight break. On the acoustic waveform, the cursor was placed on each word in the passage to be analysed and was assigned a frequency value. These values were added together then divided by the number of words in the sentence, giving an average frequency for each passage. All three passages were analysed in this way, and the average from each reading added together and divided by three to give an overall average fundamental pitch $\left(F_{0}\right)$ of the spoken voice (Speaking fundamental frequency SFF). 


\subsection{Ultrasound Data}

The following ultrasound measurements were recorded during normal quiet respiration:

- Height $(\mathrm{mm})$ of the cricoid and thyroid cartilages in the midline

- Cricothyroid space $(\mathrm{mm})$, measured in the midline between the anteroinferior edge of the thyroid cartilage and the anterosuperior edge of the cricoid cartilage

- Ultrasound unit and transducer

A Siemens ACUSON Sequoia 512 (Mountainview, CA) ultrasound machine with a 8-15 MHz multifrequency linear-array transducer (15L8w) was used. The transducer is specifically designed for high resolution scanning of superficial structures. The high-definition zoom function was used to enlarge selected images for improved visualization. Standard calibrated electronic calipers were used to make distance measurements. Ultrasound images were recorded on a DICOM data DVD and numeric data entered into a Microsoft Excel 2010 spreadsheet (Microsoft Corporation, product version 14.0.6029.1000).

- Ultrasound Technique and Measurements

Ultrasound examinations were performed in the Department of Ultrasound, Radiology Department at Waikato Hospital, Hamilton, New Zealand. Each investigation was carried out by the same experienced sonographer. Participants were seated and the transducer was placed on the skin anteriorly over the larynx in the mid-sagittal plane (Figure 5.8). The linear echogenic band between the anteroinferior border of the thyroid cartilage and the anterosuperior edge of the cricoid cartilage was measured to define the cricothyroid space (Singh, et al., 2010). 


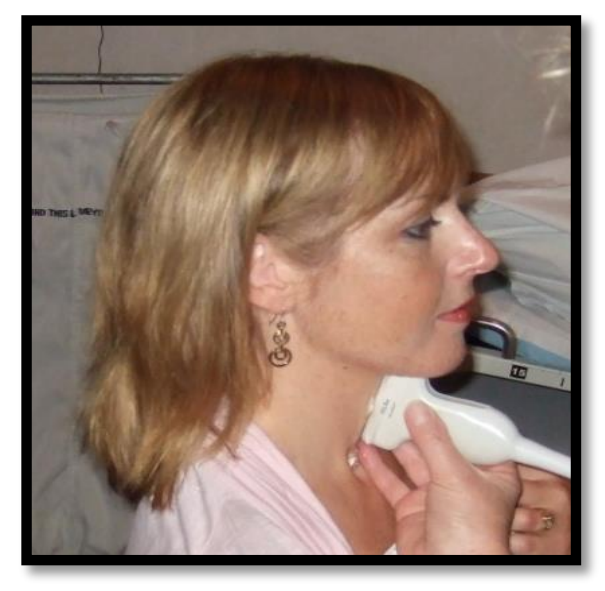

Figure 5.8: Illustrates the examination procedure (Source: Author)

Figure 5.9 shows an ultrasound image of the larynx with associated measurements. Cricoid cartilage height was measured between points 1 and 2 and is shown as distance 1 coloured blue in the right hand corner. Similarly, the cricothyroid space was measured between points 2 and 3 , and is distance 2 coloured green. Measurement between the number 3 points represents the height of the thyroid cartilage and is distance 3 coloured pink.

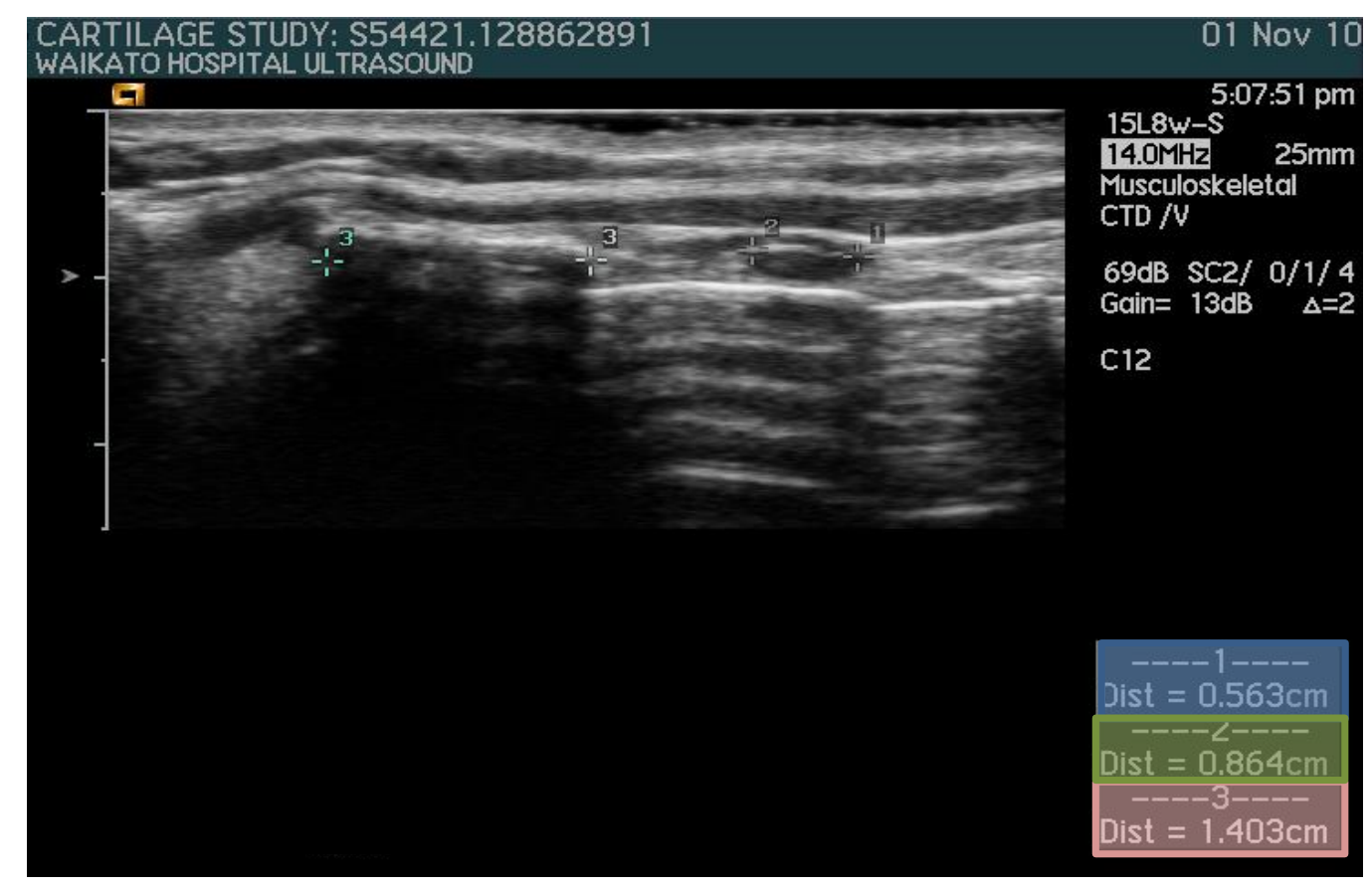

Figure 5.9: Longitudinal sagittal ultrasound image of larynx with measurements. 1-2 = cricoid cartilage; $2-3$ = cricothyroid space; $3-3$ the thyroid cartilage (Source: Author) 


\subsection{Statistical analysis}

Statistical analysis of laryngeal dimensions, anthropometric data and vocal data was performed using analysis of variance (ANOVA; Minitab version 16.2.2 [2010] analytical software).

The Student's 2 sample t-test was used to compare the means of the two normally distributed continuous variables (soprano and mezzo-soprano) from separate samples (age, ethnicity, anthropometric indices, neck dimensions, vocal data). Whereas the paired Students t-test was used to asses differences between intra-rater measurements.

Continuous variables were analysed with Pearson's product-moment correlation co-efficient ( $P$ value). This is typically denoted by $r$ and is a measure of the correlation (linear dependence) between two variables (Swinscow \& Campbell, 2002). All tests were performed as two-sided analyses, taking $\mathrm{P}<0.05$ as statistically significant.

Relationships between variables were analysed using linear regression, with categorical predictors being included where it was useful to test whether relationships depended on category. 


\subsection{Ethical Approval}

Any research involving human participants must be conducted in accordance with the highest ethical standards. The research project in this thesis complies with all of the requirements of the University of Waikato's ethics policy. The investigation of the cricothyroid interval in human participants was approved by the Faculty of Arts and Social Sciences (FASS) Human Research Ethics Committee on 26 August 2010 (FS201033). Appendix F contains material relating to the approval and participant consent process. 


\section{Results}

The study group comprised 43 women: 33 sopranos (4 coloraturas, 6 spintos, 10 light lyric sopranos, 5 heavy lyric sopranos, 4 soubrettes, 3 dramatic sopranos) and 11 mezzo-sopranos. Among the participants was one set of fraternal twins, a mother and daughter, one singer with a cleft palate that had been surgically corrected along with a jaw (mandibular) advancement, and two participants who had previously been diagnosed with Graves' disease (thyrotoxicosis), both currently off treatment.

A summary of all measurements including general anthropometric indices, and laryngeal cartilage dimensions are shown in Table 5. (Appendix $G$ contains a summary of general anthropometric indices and laryngeal cartilage dimensions divided into voice types: soprano and mezzosoprano).

Table 5: Anthropometric indices, laryngeal cartilage dimensions, and the cricothyroid space data for the study sample

\begin{tabular}{|c|c|c|c|c|}
\hline Parameter & $\begin{array}{c}\text { Mean } \\
\mathbf{( n = 4 3 )}\end{array}$ & $\begin{array}{c}\text { SD } \\
(\mathbf{n = 4 3})\end{array}$ & SE mean & $\begin{array}{c}\text { Range } \\
(\mathbf{n = 4 3})\end{array}$ \\
\hline Age (years) & 38.1 & 14.3 & 2.2 & $18-63$ \\
\hline Height (cm) & 165.7 & 6.7 & 1.0 & $151.3-177$ \\
\hline Weight (kg) & 73.0 & 18.2 & 2.8 & $47.4-139.8$ \\
\hline BMI (kg/m²) & 26.5 & 5.8 & 0.9 & $19.3-44.6$ \\
\hline Neck circumference (cm) & 34.0 & 3.2 & 0.5 & $30.0-45.5$ \\
\hline $\begin{array}{c}\text { Neck length (cm) } \\
\text { Cricoid cartilage arch height in } \\
\text { midline (mm) }\end{array}$ & 12.1 & 1.5 & 0.2 & $9.0-15.4$ \\
\hline Cricothyroid space (mm) & 10.7 & 1.4 & 0.2 & $7.3-14.3$ \\
\hline $\begin{array}{c}\text { Thyroid cartilage height in midline } \\
\text { (mm) }\end{array}$ & 12.1 & 2.0 & 0.3 & $8.3-16.2$ \\
\hline $\begin{array}{c}\text { Combined height of thyroid and } \\
\text { cricoid cartilages \& cricothyroid } \\
\text { space in midline (mm) }\end{array}$ & 28.5 & 2.9 & 0.4 & $21.8-35.0$ \\
\hline
\end{tabular}


A summary of all acoustic measurements including lowest and highest performance notes, lowest and highest practice notes, performance and practice vocal ranges are shown in Table 6 . As would be expected the lowest and highest performance notes and performance vocal range is smaller than the lowest and highest practice notes and practice vocal range. (Appendix $\mathrm{H}$ contains a summary of acoustic data divided into voice types: soprano and mezzo-soprano).

Table 6: Acoustic data of the study sample

\begin{tabular}{|c|c|c|c|c|}
\hline Parameter & $\begin{array}{l}\text { Mean } \\
(n=43)\end{array}$ & $\begin{array}{c}\text { SD } \\
(n=43)\end{array}$ & SE mean & $\begin{array}{l}\text { Range } \\
(n=43)\end{array}$ \\
\hline $\begin{array}{c}\text { Speaking fundamental } \\
\text { frequency } \\
\text { SFF }(\mathrm{Hz})\end{array}$ & 200 & 26.5 & 4.1 & $152-243$ \\
\hline $\begin{array}{c}\text { Lowest performance } \\
\text { note } \\
\text { (pitch) }\end{array}$ & F\#3 & 2.6 & 0.4 & $\mathrm{C} 3-\mathrm{A} \# 3$ \\
\hline $\begin{array}{c}\text { Highest performance } \\
\text { note } \\
\text { (pitch) }\end{array}$ & $\mathrm{C \# 6}$ & 2.4 & 0.4 & G\#5- F6 \\
\hline $\begin{array}{l}\text { Performance vocal } \\
\text { range (semitones) }\end{array}$ & 31 & 3.1 & 0.5 & $24-39$ \\
\hline $\begin{array}{l}\text { Lowest practice note } \\
\text { (pitch) }\end{array}$ & D\#3 & 2.8 & 0.4 & $\mathrm{G} 2-\mathrm{G} 3$ \\
\hline $\begin{array}{l}\text { Highest practice note } \\
\text { (pitch) }\end{array}$ & E6 & 2.8 & 0.4 & A\#5- F6 \\
\hline $\begin{array}{l}\text { Practice vocal range } \\
\text { (semitones) }\end{array}$ & 37 & 3.7 & 0.6 & $29-46$ \\
\hline
\end{tabular}




\section{- Ethnicity}

In 39 of the 43 participants in this study both parents were NZ European. Three of the remaining four participants had one NZ European and one NZ Māori parent, and the other had two NZ Māori parents. However, it should be noted that ethnicity is self-defined and is primarily a cultural affiliation and should not be confused with race (Statistics New Zealand, 2013).

- Age

Mean age was $38.1 \pm 14.3$ years (range 18-63 years). There were 24 participants under the age of 40 years, and 19 aged 40 years or more. There were no significant differences in age between the different voice types (Figure 6.1).

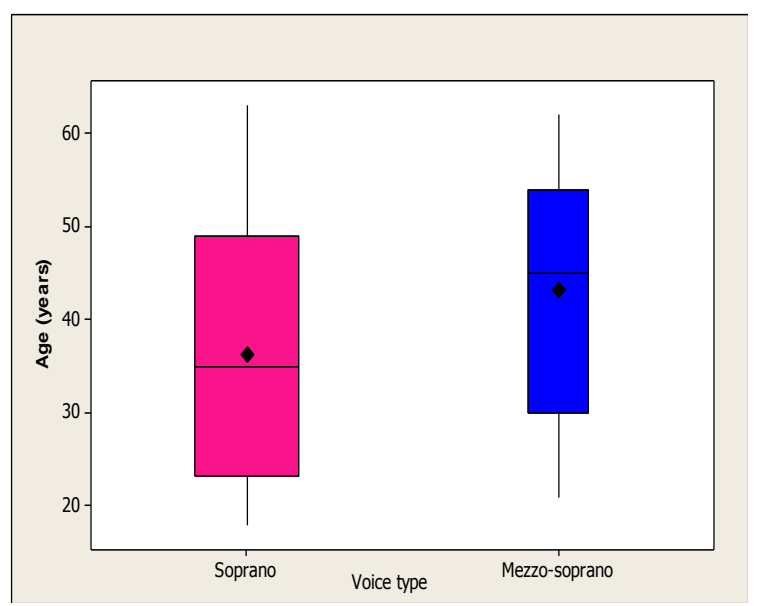

\begin{tabular}{|c|c|c|}
\hline $\begin{array}{c}\text { Voice } \\
\text { type }\end{array}$ & $\mathbf{n}$ & $\begin{array}{c}\text { Mean age } \\
\text { (years) }\end{array}$ \\
\hline All & 43 & $38.1 \pm 14.3$ \\
\hline Soprano & 32 & $36.3 \pm 14.4$ \\
\hline $\begin{array}{c}\text { Mezzo- } \\
\text { soprano }\end{array}$ & 11 & $43.2 \pm 13.4$ \\
\hline \multicolumn{2}{|c|}{ Significance (P) } & 0.165 \\
\hline
\end{tabular}

Figure 6.1: Comparison of age for each voice type shown as box and whisker plot $^{5}$ and tabular mean values

\footnotetext{
${ }^{5}$ Box and whisker plots are used to summarise and compare sample distributions. A box and whisker plot displays the median, quartiles, and minimum and maximum observations for a group. The box plot provides a visual summary of the data and identifies outliers. The mean value is displayed as a diamond $(\downarrow)$. The centre horizontal line in the box represents the sample median or middle of the data. The bottom and top edges of the box correspond to the 25th (Quartile1) and 75th (Quartile 3) percentiles. The box length is therefore one interquartile range (Quartile 3 Quartile 1). The vertical lines that project out from the box, called whiskers, extend up to a distance of 1.5 of the box (interquartile ranges). The upper whisker extends to the highest data value within the upper limit and the lower whisker extends to the lowest value within the lower limit.
} 
- Height

Mean height was $165.7 \pm 6.7 \mathrm{~cm}$ (range 151.3-177 cm). There was a tendency for the mezzo-sopranos to be taller than sopranos, but this was not statistically significant (Figure 6.2).

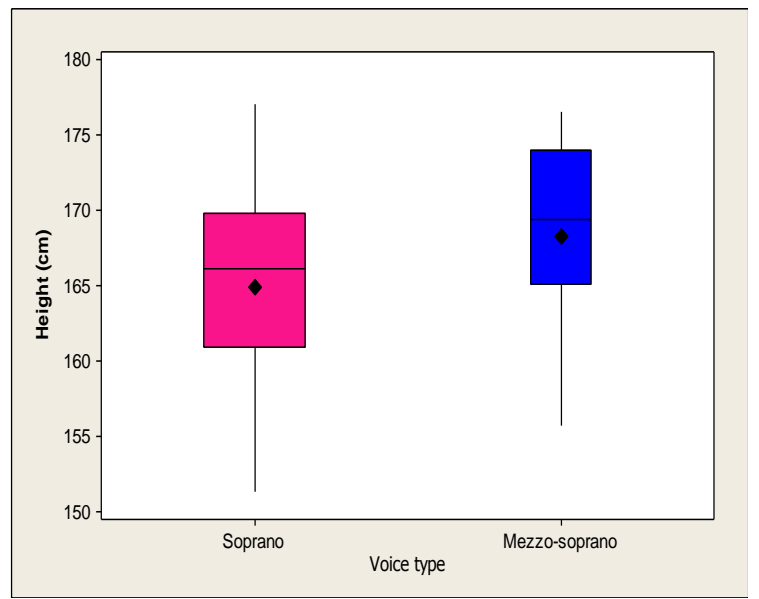

\begin{tabular}{|c|c|c|}
\hline $\begin{array}{c}\text { Voice } \\
\text { type }\end{array}$ & $\mathbf{n}$ & $\begin{array}{c}\text { Mean height } \\
\text { (cm) }\end{array}$ \\
\hline All & 43 & $165.7 \pm 6.7$ \\
\hline Soprano & 32 & $164.9 \pm 6.6$ \\
\hline $\begin{array}{c}\text { Mezzo- } \\
\text { soprano }\end{array}$ & 11 & $168.2 \pm 6.8$ \\
\hline \multicolumn{2}{|c|}{ Significance (P) } & 0.175 \\
\hline
\end{tabular}

Figure 6.2: Comparison of height for each voice type as box and whisker plot and tabular mean values

- Weight

Mean weight was $73.0 \pm 18.2 \mathrm{~kg}$ (range 47.4 - $139.8 \mathrm{~kg}$ ). The mean mezzo-soprano weight was slightly heavier than sopranos but this was not statistically significant (Figure 6.3).

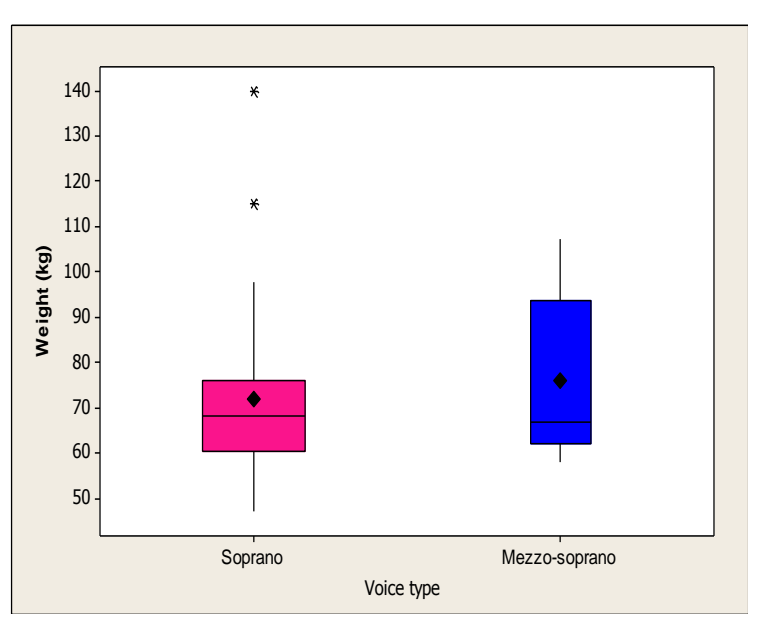

\begin{tabular}{|c|c|c|}
\hline $\begin{array}{c}\text { Voice } \\
\text { type }\end{array}$ & $\mathbf{n}$ & $\begin{array}{c}\text { Mean } \\
\text { weight } \\
\mathbf{( k g )}\end{array}$ \\
\hline All & 43 & $73.0 \pm 18.2$ \\
\hline Soprano & 32 & $71.9 \pm 18.7$ \\
\hline $\begin{array}{l}\text { Mezzo- } \\
\text { soprano }\end{array}$ & 11 & $76.1 \pm 16.7$ \\
\hline \multicolumn{2}{|c|}{ Significance (P) } & 0.507 \\
\hline
\end{tabular}

Figure 6.3: Comparison of weight for each voice type as box and whisker plot ${ }^{6}$ and tabular mean values

${ }^{6}$ Values beyond the whiskers are outliers, which are unusually large or small observations. These extreme values are designated with a cross $(x)$ 
- Body mass index

Mean body mass index (BMI) was $26.5 \pm 5.8 \mathrm{~kg} / \mathrm{m}^{2}$ with a range of 19.3 $44.6 \mathrm{~kg} / \mathrm{m}^{2}$. Average BMl showed no significant difference for both voice types (Figure 6.4).

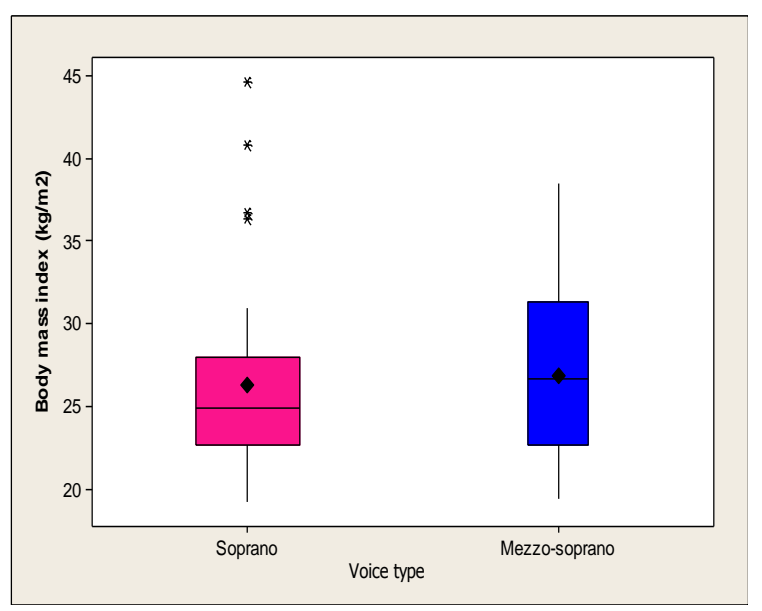

\begin{tabular}{|c|c|c|}
\hline $\begin{array}{c}\text { Voice } \\
\text { Type }\end{array}$ & $\mathbf{n}$ & $\begin{array}{c}\text { Mean BMI } \\
\mathbf{~ k g} / \mathbf{m}^{2}\end{array}$ \\
\hline All & 43 & $26.5 \pm 5.8$ \\
\hline Soprano & 32 & $26.4 \pm 6.0$ \\
\hline $\begin{array}{c}\text { Mezzo- } \\
\text { soprano }\end{array}$ & 11 & $26.9 \pm 5.6$ \\
\hline \multicolumn{2}{|l|}{ Significance (P) } & 0.807 \\
\hline
\end{tabular}

Figure 6.4: Comparison of BMI for each voice type as box and whisker plot and tabular mean values

- Neck circumference

Mean neck circumference was $34.0 \pm 3.2(30.0-45.5) \mathrm{cm}$. The average mean neck circumference was exactly the same for sopranos and mezzosopranos $(\mathrm{P}=0.993)$ (Figure 6.5).

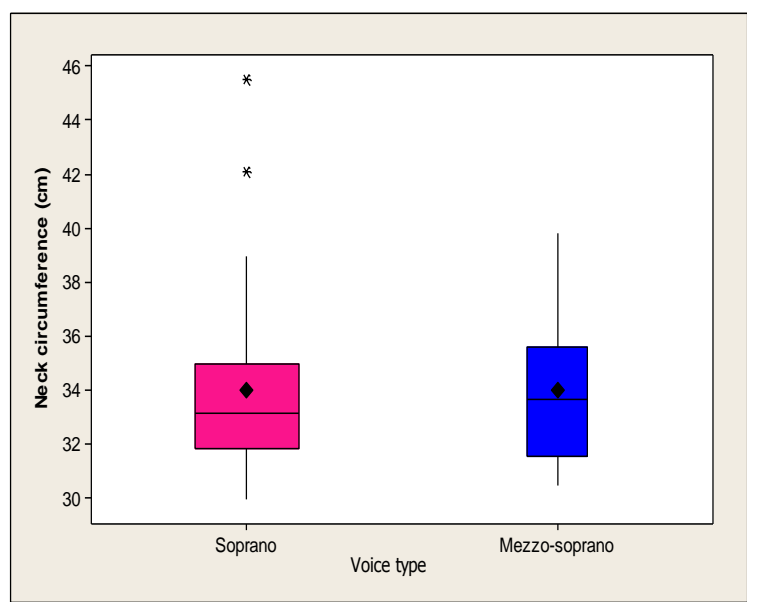

\begin{tabular}{|c|c|c|}
\hline $\begin{array}{c}\text { Voice } \\
\text { Type }\end{array}$ & $\mathbf{n}$ & $\begin{array}{c}\text { Mean neck } \\
\text { circumference } \\
\text { (cm) }\end{array}$ \\
\hline All & 43 & $34.0 \pm 3.2$ \\
\hline Soprano & 32 & $34.0 \pm 3.4$ \\
\hline $\begin{array}{c}\text { Mezzo- } \\
\text { soprano }\end{array}$ & 11 & $34.0 \pm 2.7$ \\
\hline $\begin{array}{c}\text { Significance } \\
(\mathrm{P})\end{array}$ & 0.993 \\
\hline
\end{tabular}

Figure 6.5: Comparison of neck circumference for each voice type as box and whisker plot and tabular mean values 
- Neck length

Mean neck length was $12.1 \pm 1.5 \mathrm{~cm}$ (range 9.0-15.4 cm). Even though there was a tendency for the mezzo-sopranos to have a longer neck than the sopranos, this was just short of statistical significance (Figure 6.6).

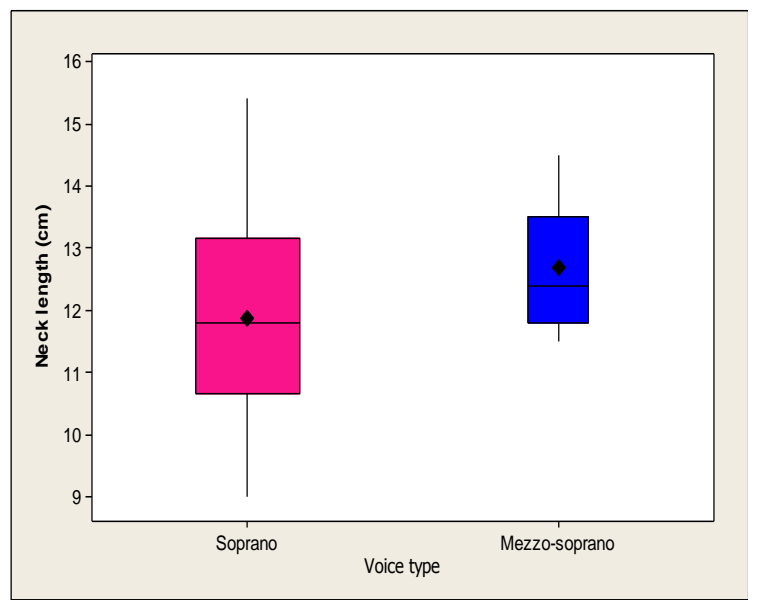

\begin{tabular}{|c|c|c|}
\hline $\begin{array}{c}\text { Voice } \\
\text { Type }\end{array}$ & $\mathbf{n}$ & $\begin{array}{c}\text { Mean neck } \\
\text { length }(\mathbf{c m})\end{array}$ \\
\hline All & 43 & $12.1 \pm 1.5$ \\
\hline Soprano & 32 & $11.9 \pm 1.7$ \\
\hline $\begin{array}{l}\text { Mezzo- } \\
\text { soprano }\end{array}$ & 11 & $12.7 \pm 1.0$ \\
\hline \multicolumn{2}{|l|}{ Significance (P) } & 0.061 \\
\hline
\end{tabular}

Figure 6.6: Comparison of neck length for each voice type as box and whisker plot and tabular mean values

- Cricoid cartilage

Mean cricoid cartilage arch height in the midline was $5.7 \pm 0.7 \mathrm{~mm}$ with a range from $4.4-7.3 \mathrm{~mm}$. The cricoid cartilage measurements were very similar between the sopranos and mezzo-sopranos (Figure 6.7).

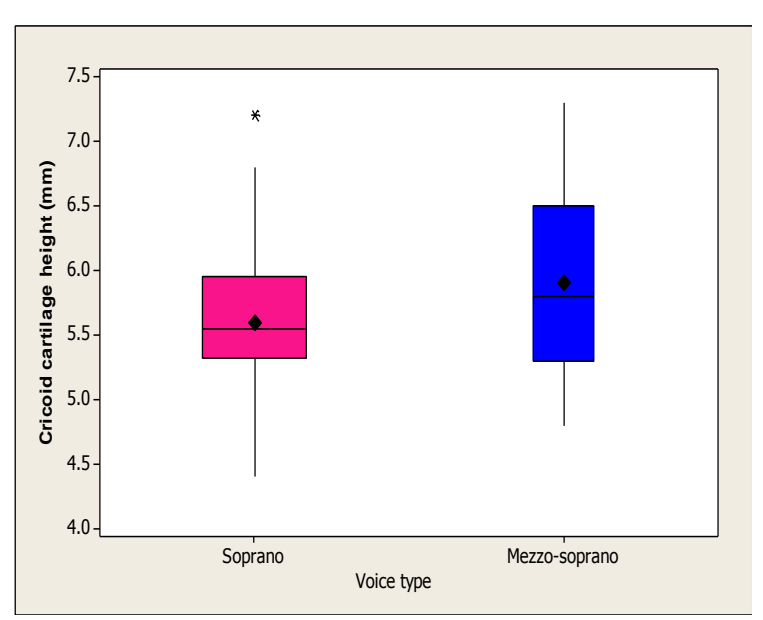

\begin{tabular}{|c|c|c|}
\hline $\begin{array}{c}\text { Voice } \\
\text { Type }\end{array}$ & $\mathbf{n}$ & $\begin{array}{c}\text { Mean cricoid } \\
\text { cartilage } \\
(\mathbf{m m})\end{array}$ \\
\hline All & 43 & $5.7 \pm 0.7$ \\
\hline Soprano & 32 & $5.6 \pm 0.7$ \\
\hline $\begin{array}{c}\text { Mezzo- } \\
\text { soprano }\end{array}$ & 11 & $5.9 \pm 0.7$ \\
\hline \multicolumn{2}{|l|}{ Significance (P) } & 0.213 \\
\hline
\end{tabular}

Figure 6.7: Comparison of cricoid cartilage arch height for each voice type as box and whisker plot and tabular mean values 
- Cricothyroid space

The mean cricothyroid space was measured at $10.7 \pm 1.4 \mathrm{~mm}$ ranging from 7.3-14.0 $\mathrm{mm}$. When the cricothyroid space was divided into voice types, this showed a statistically significant difference between the two groups ( $P=0.007$ ) (Figure 6.8). Mezzo-sopranos had a larger cricothyroid space than sopranos.

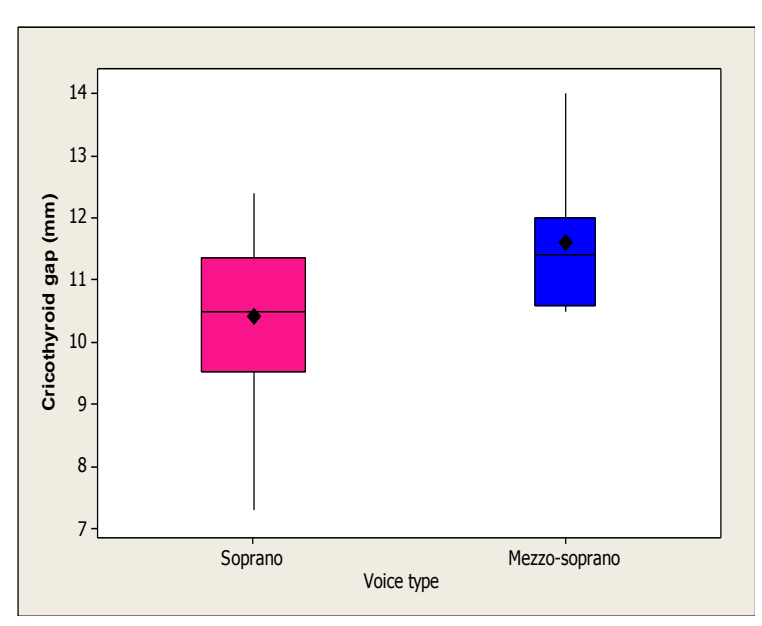

\begin{tabular}{|c|c|c|}
\hline $\begin{array}{c}\text { Voice } \\
\text { Type }\end{array}$ & $\mathbf{n}$ & $\begin{array}{c}\text { Mean } \\
\text { cricothyroid } \\
\text { space } \\
(\mathbf{m m})\end{array}$ \\
\hline All & 43 & $10.7 \pm 1.4$ \\
\hline Soprano & 32 & $10.4 \pm 1.4$ \\
\hline $\begin{array}{l}\text { Mezzo- } \\
\text { soprano }\end{array}$ & 11 & $11.6 \pm 1.1$ \\
\hline \multicolumn{2}{|c|}{ Significance (P) } & 0.007 \\
\hline
\end{tabular}

Figure 6.8: Comparison of the cricothyroid space dimensions for each voice type as box and whisker plot and tabular mean values

The sopranos CT space measurement ranged from 7.3-12.4 mm, whilst the mezzo-sopranos range was from $10.5-14.0 \mathrm{~mm}$. Figure 6.9 shows that all singers with a resting CT space of less than $10.4 \mathrm{~mm}$ were sopranos (comprising 15 out of the 32 sopranos), whilst all singers with a resting CT space of greater than $13.0 \mathrm{~mm}$ were mezzo-sopranos. Individuals with resting CT spaces between 10.5-12.4 mm had mixed voice types. The type of soprano, namely lyric, coloratura, spinto etc. did not appear to be predictable from the CT space measurement (Figure 6.10). 


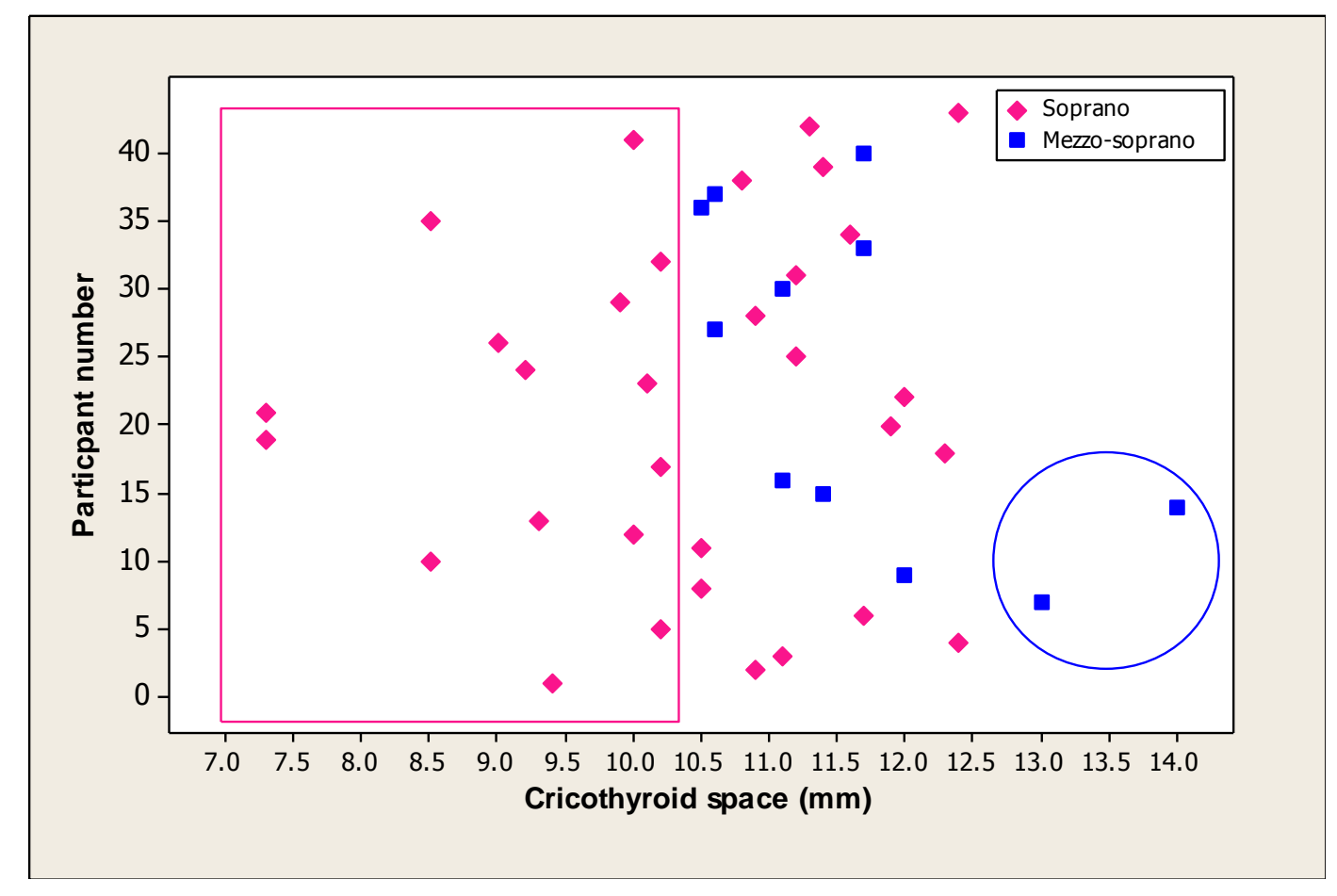

Figure 6.9: Cricothyroid space measurements divided into soprano and mezzosoprano. The pink rectangle shows sopranos with a resting CT space of less than $10.4 \mathrm{~mm}$, whilst the blue circle depicts mezzo-sopranos with a resting CT space of greater than $13 \mathrm{~mm}$

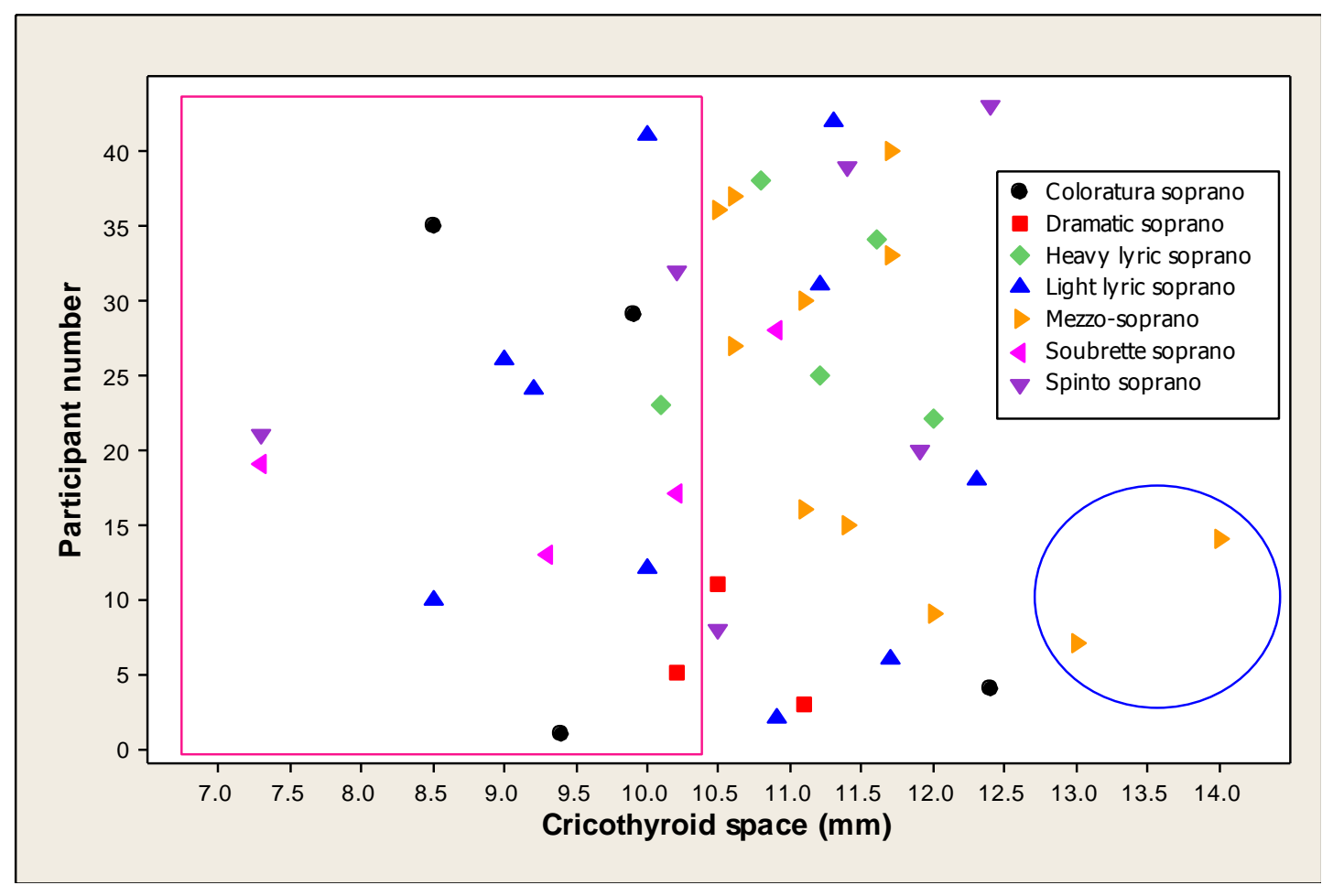

Figure 6.10: Cricothyroid space measurements according to Fach. The pink rectangle shows the Fach of those sopranos with a resting CT space of less than $10.4 \mathrm{~mm}$, whilst the blue circle depicts the mezzo-sopranos with a resting CT space of greater than $13 \mathrm{~mm}$ 
- Thyroid cartilage measurement

The mean thyroid cartilage height in the midline was $12.1 \pm 2.0 \mathrm{~mm}$ (range $8.3-16.2 \mathrm{~mm}$ ). There was no significant difference between the two voice types (Figure 6.11).

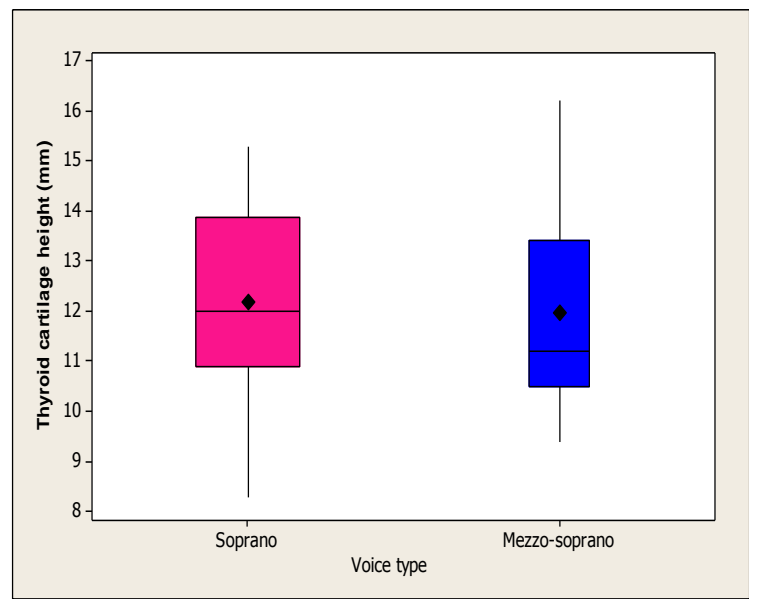

\begin{tabular}{|c|c|c|}
\hline $\begin{array}{c}\text { Voice } \\
\text { Type }\end{array}$ & $\mathbf{n}$ & $\begin{array}{c}\text { Mean thyroid } \\
\text { cartilage } \\
(\mathbf{m m})\end{array}$ \\
\hline All & 43 & $12.1 \pm 2.0$ \\
\hline Soprano & 31 & $12.2 \pm 2.0$ \\
\hline $\begin{array}{c}\text { Mezzo- } \\
\text { soprano }\end{array}$ & 12 & $12.0 \pm 2.2$ \\
\hline \multicolumn{2}{|c|}{ Significance (P) } & 0.769 \\
\hline
\end{tabular}

Figure 6.11: Comparison of thyroid cartilage height for each voice type as box and whisker plot and tabular mean values

- Total length of laryngeal cartilages

The combined length of the thyroid and cricoid cartilages and cricothyroid space in the midline was $28.5 \pm 2.9 \mathrm{~mm}$ ranging from 21.8 to $35.0 \mathrm{~mm}$. There was no significant difference between sopranos and mezzosopranos (Figure 6.12).

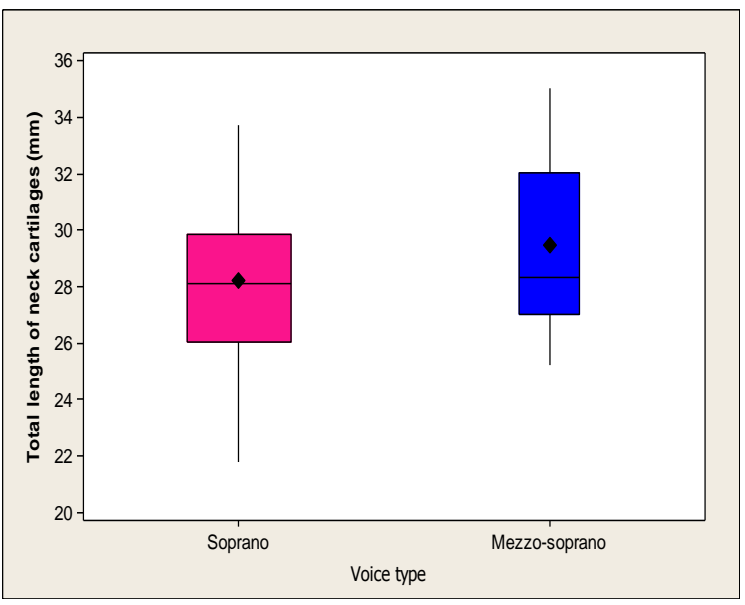

\begin{tabular}{|c|c|c|}
\hline $\begin{array}{c}\text { Voice } \\
\text { Type }\end{array}$ & $\mathbf{n}$ & $\begin{array}{c}\text { Mean total } \\
\text { length of } \\
\text { laryngeal } \\
\text { cartilages } \\
(\mathbf{m m})\end{array}$ \\
\hline All & 43 & $28.5 \pm 2.9$ \\
\hline Soprano & 31 & $28.2 \pm 2.9$ \\
\hline $\begin{array}{l}\text { Mezzo- } \\
\text { soprano }\end{array}$ & 12 & $29.5 \pm 3.1$ \\
\hline \multicolumn{2}{|l|}{ Significance (P) } & 0.248 \\
\hline
\end{tabular}

Figure 6.12: Comparison of combined length of laryngeal cartilages in the midline for each voice type as box and whisker plot and tabular mean values 
- Speaking fundamental frequency

The overall mean speaking fundamental frequency was $200 \pm 36.5 \mathrm{~Hz}$, ranging from $152-243 \mathrm{~Hz}$. As expected, sopranos had a significantly higher mean SFF than mezzo-sopranos (Figure 6.13).

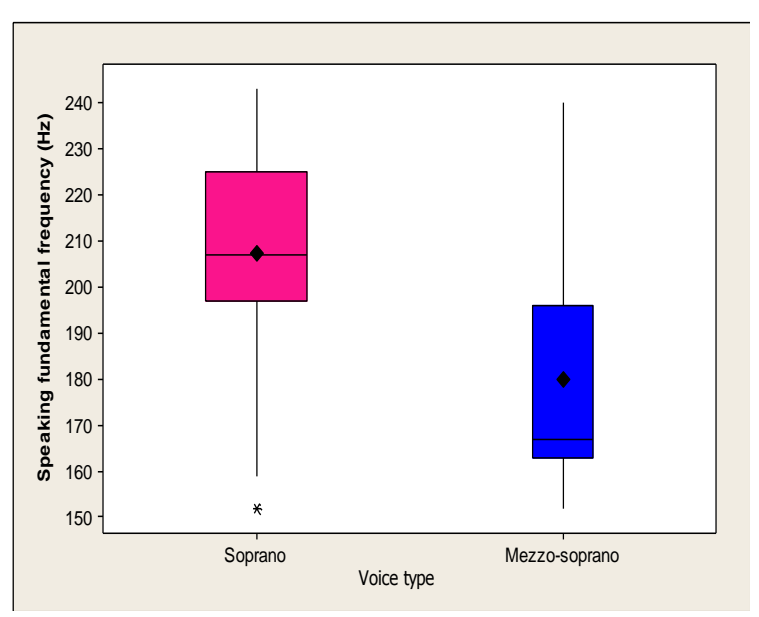

\begin{tabular}{|c|c|c|}
\hline $\begin{array}{c}\text { Voice } \\
\text { Type }\end{array}$ & $\mathbf{n}$ & $\begin{array}{c}\text { Mean SFF } \\
\mathbf{( H z )}\end{array}$ \\
\hline All & 43 & $200 \pm 36.5$ \\
\hline Soprano & 32 & $207 \pm 23.1$ \\
\hline $\begin{array}{c}\text { Mezzo- } \\
\text { soprano }\end{array}$ & 11 & $180 \pm 26.3$ \\
\hline \multicolumn{2}{|c|}{ Significance (P) } & 0.008 \\
\hline
\end{tabular}

Figure 6.13: Comparison of speaking fundamental frequency for each voice type as box and whisker plot and tabular mean values

Three of the 43 participants had one NZ European and one NZ Māori parent, and one had two NZ Māori parents; their SFFs ranged from 163 to $227 \mathrm{~Hz}$.

There was no significant difference in average SFF for participants under 40 years of age compared to older participants (Table 7 ).

Table 7: Comparison of speaking fundamental frequency in participants younger or older than 40 years of age.

\begin{tabular}{|c|c|c|}
\hline $\begin{array}{c}\text { Age } \\
\text { (years) }\end{array}$ & $\mathbf{n}$ & $\begin{array}{c}\text { Mean SFF } \\
\mathbf{( H z )}\end{array}$ \\
\hline $18-39$ & 24 & $202 \pm 21.5$ \\
\hline $40-63$ & 19 & $199 \pm 32.4$ \\
\hline \multicolumn{2}{|c|}{ Significance (P) } & 0.744 \\
\hline
\end{tabular}


The lowest SFF of $152 \mathrm{~Hz}$ was observed in two participants aged 40 and 56 years, whilst the highest SFF of $243 \mathrm{~Hz}$ was achieved by a 49 year old. The two oldest participants were 63 years. One of them had the second highest SFF of $240 \mathrm{~Hz}$, whilst the other had a SFF of $203 \mathrm{~Hz}$. Conversely, the youngest participant, who was 18 years old, had a SFF of $167 \mathrm{~Hz}$. (See Appendix I for raw data). In this study there was one pair of dizygotic twins and a mother and daughter. The SFF results in the twins (both sopranos) were $222 \mathrm{~Hz}$ and $214 \mathrm{~Hz}$, whilst the SFFs from mother and daughter (both sopranos) were 243 and $239 \mathrm{~Hz}$, respectively.

- Lowest performance note

As expected, sopranos had significantly higher lower performance notes (G3) than mezzo-sopranos (E3) (Figure 6.14).

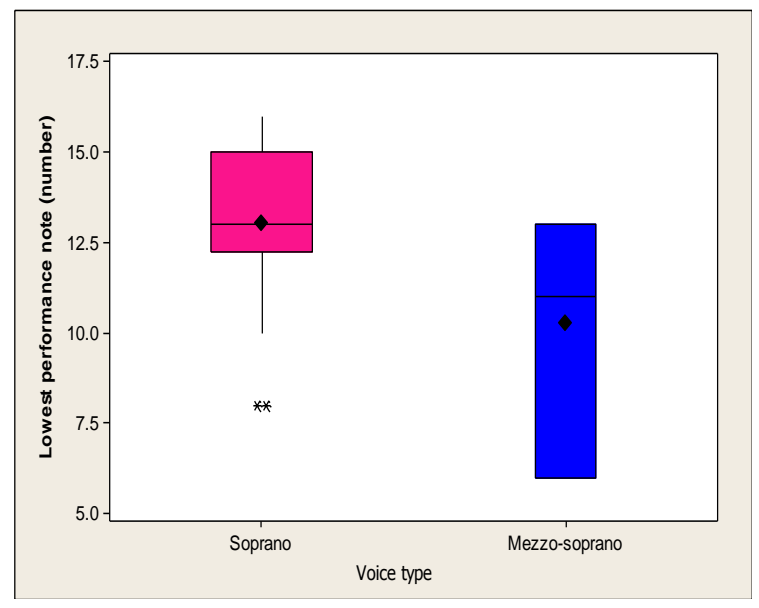

\begin{tabular}{|c|c|c|}
\hline $\begin{array}{c}\text { Voice } \\
\text { Type }\end{array}$ & $\mathbf{n}$ & $\begin{array}{c}\text { Mean lowest } \\
\text { performance } \\
\text { note }\end{array}$ \\
\hline All & 43 & F\#3 \pm 2.6 \\
\hline Soprano & 32 & G3 \pm 2.1 \\
\hline $\begin{array}{c}\text { Mezzo- } \\
\text { soprano }\end{array}$ & 11 & E3 \pm 3.0 \\
\hline \multicolumn{2}{|l|}{ Significance (P) } & 0.013 \\
\hline
\end{tabular}

Figure 6.14: Comparison of lowest performance note for each voice type as box and whisker plot and tabular mean values (Appendix $\mathrm{J}$ contains a Table for converting musical note names into numbers).

In Figure 6.15 it can be seen that sopranos had a range from D3 - A\#3 (numbers 8 -16) for their lowest performance notes, whilst for mezzosopranos the range was from C3 - G3 (numbers 6-13). Figure 6.15 clearly shows that 12 of the 32 sopranos had lowest performance notes between $\mathrm{G} \# 3$ and $A \# 3$ (numbers 14-16 in pink rectangle) whilst three of 11 mezzosopranos had C3 as their lowest performance note (number 6 in blue oval). Between these groups was a mixture of sopranos and mezzo-sopranos. 


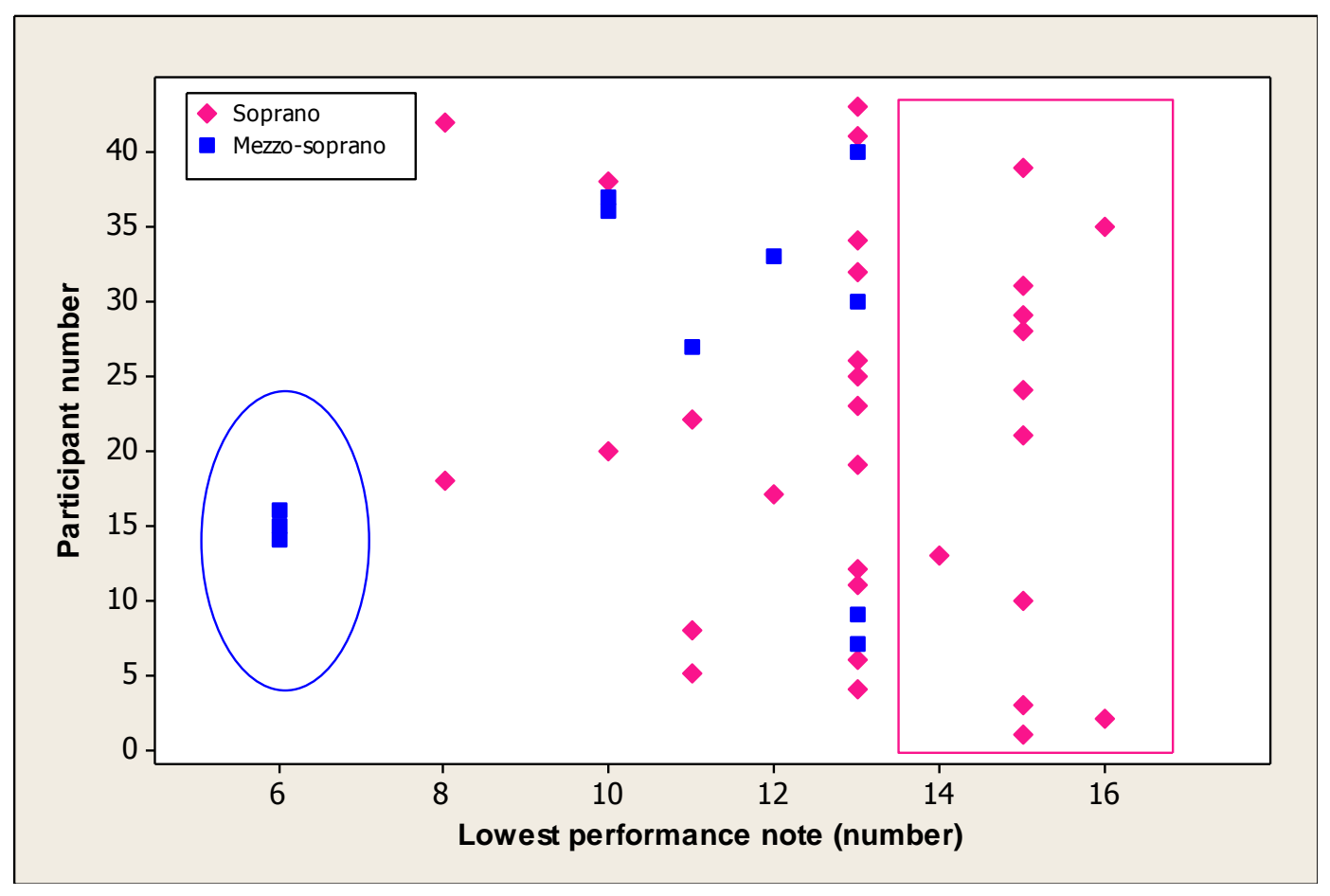

Figure 6.15: Relationship of lowest performance note to voice type

- Highest performance note

Not surprisingly, sopranos had a significantly higher mean performance note (D6) than mezzo-sopranos (A\#5) (Figure 6.16).

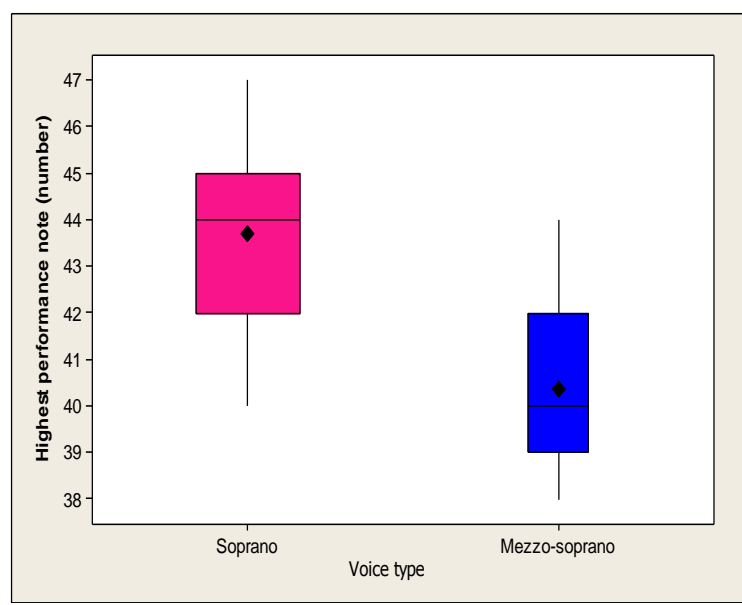

\begin{tabular}{|c|c|c|}
\hline $\begin{array}{c}\text { Voice } \\
\text { Type }\end{array}$ & $\mathbf{n}$ & $\begin{array}{c}\text { Mean } \\
\text { highest } \\
\text { performance } \\
\text { note }\end{array}$ \\
\hline All & 43 & $\mathrm{C \# 6} \pm 2.4$ \\
\hline Soprano & 32 & $\mathrm{D} 6 \pm 2.0$ \\
\hline $\begin{array}{c}\text { Mezzo- } \\
\text { soprano }\end{array}$ & 11 & $\mathrm{~A} \# 5 \pm 1.9$ \\
\hline \multicolumn{2}{|l|}{ Significance (P) } & $<0.0005$ \\
\hline
\end{tabular}

Figure 6.16: Comparison of highest performance note for each voice type as box and whisker plot and tabular mean values 
Figure 6.17 clearly shows that the majority of sopranos had their highest performance note above C\#6 (number 43). There was one mezzo-soprano with a highest performance note of D6 (number 44). Mezzo-sopranos had lower highest performance notes, around G\#5 to A5 (numbers 38/39). As expected there was an overlap in the middle where sopranos and mezzosopranos had highest performance notes around A\#5 to C6 (numbers 4042).

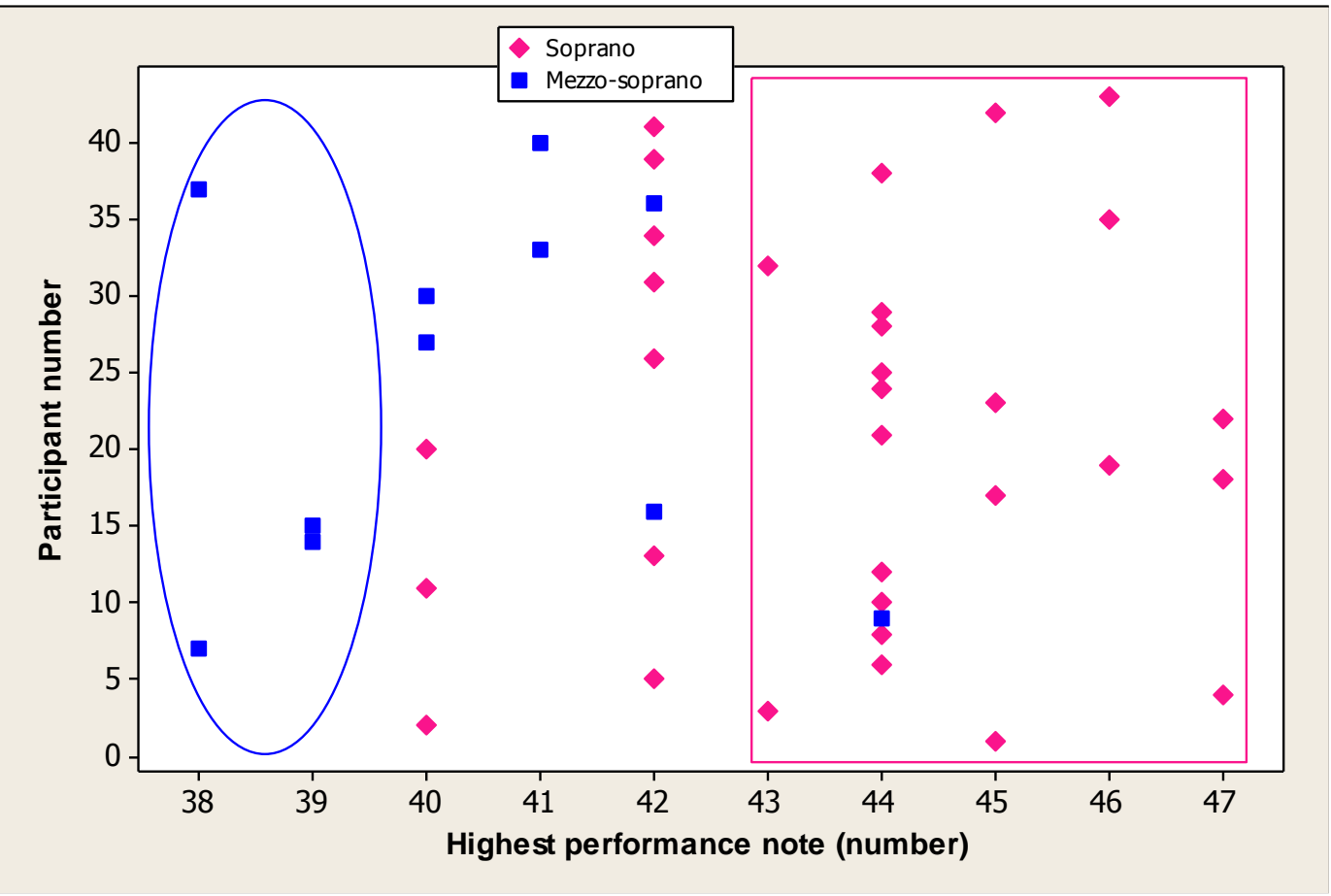

Figure 6.17: Relationship of highest performance note to voice type. The pink rectangle shows the sopranos who had highest performance notes above C\#6 (number 43), whilst the blue oval depicts the mezzo-sopranos whose highest performance notes were between G\#5 and A5 (numbers 38 and 39) 
- Performance vocal range

The performance vocal range, which is accepted as the musical performance range, was similar between sopranos and mezzo-sopranos (Figure 6.18).

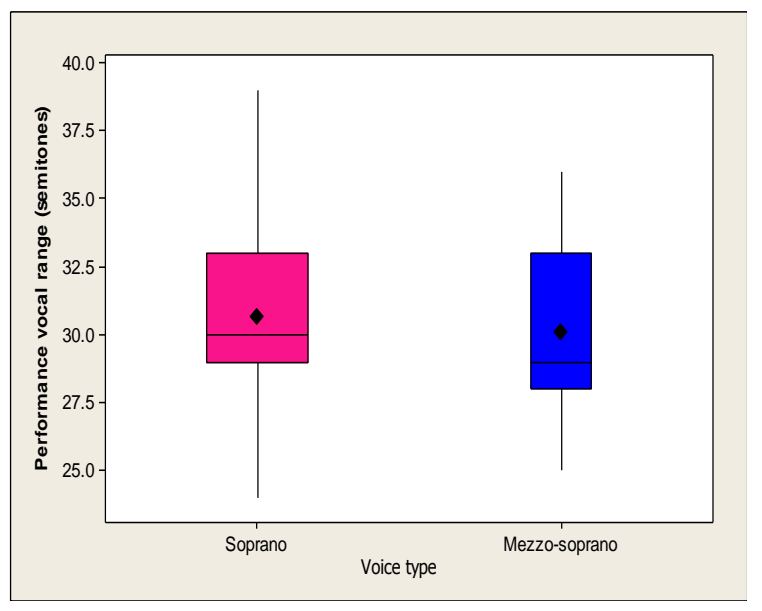

\begin{tabular}{|c|c|c|}
\hline $\begin{array}{c}\text { Voice } \\
\text { Type }\end{array}$ & $\mathbf{n}$ & $\begin{array}{c}\text { Mean } \\
\text { performance } \\
\text { vocal range }\end{array}$ \\
\hline All & 43 & $31 \pm 3.1$ \\
\hline Soprano & 32 & $31 \pm 3.2$ \\
\hline $\begin{array}{l}\text { Mezzo- } \\
\text { soprano }\end{array}$ & 11 & $30 \pm 3.2$ \\
\hline \multicolumn{2}{|c|}{ Significance (P) } & 0.619 \\
\hline
\end{tabular}

Figure 6.18: Comparison of performance vocal range for each voice type as box and whisker plot and tabular mean values

- Lowest practice note

As expected, sopranos had significantly higher mean lowest practice notes than the mezzo-sopranos (Figure 6.19).

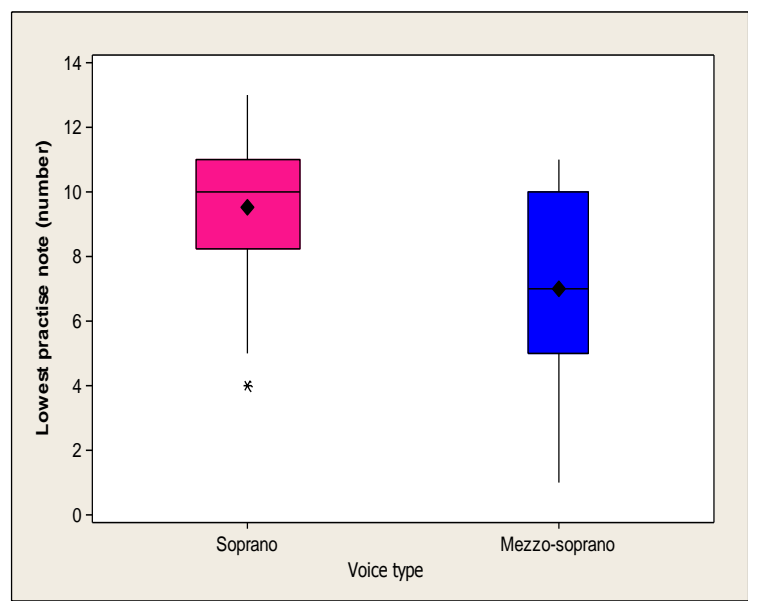

\begin{tabular}{|c|c|c|}
\hline $\begin{array}{c}\text { Voice } \\
\text { Type }\end{array}$ & $\mathbf{n}$ & $\begin{array}{c}\text { Mean lowest } \\
\text { practice } \\
\text { note }\end{array}$ \\
\hline All & 43 & $\mathrm{D \# 3} \pm 2.8$ \\
\hline Soprano & 32 & $\mathrm{E} 3 \pm 2.5$ \\
\hline $\begin{array}{c}\text { Mezzo- } \\
\text { soprano }\end{array}$ & 11 & $\mathrm{C \# 3} \pm 3.1$ \\
\hline \multicolumn{2}{|c|}{ Significance (P) } & 0.028 \\
\hline
\end{tabular}

Figure 6.19: Comparison of lowest practice note for each voice type as box and whisker plot and tabular mean values 
- Highest practice note

Also as expected the mean upper limit of the practice range highest note for the sopranos (F6) was significantly higher than for the mezzo-sopranos (C\#6) (Figure 6.20).

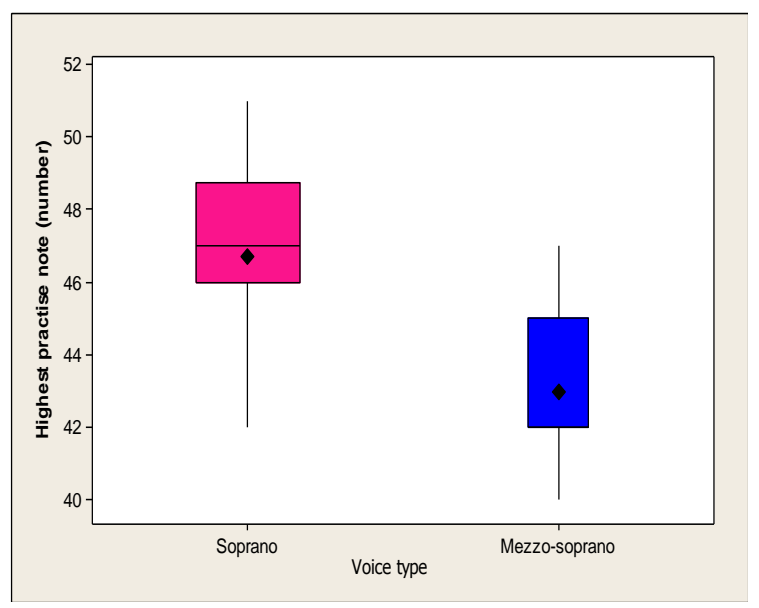

\begin{tabular}{|c|c|c|}
\hline $\begin{array}{c}\text { Voice } \\
\text { Type }\end{array}$ & $\mathbf{n}$ & $\begin{array}{c}\text { Mean } \\
\text { highest } \\
\text { practice } \\
\text { note }\end{array}$ \\
\hline All & 43 & $\mathrm{E} 6 \pm 2.8$ \\
\hline Soprano & 32 & $\mathrm{~F} 6 \pm 2.3$ \\
\hline $\begin{array}{c}\text { Mezzo- } \\
\text { soprano }\end{array}$ & 11 & $\mathrm{C \# 6} \pm 2.2$ \\
\hline \multicolumn{2}{|l|}{ Significance (P) } & $<0.0005$ \\
\hline
\end{tabular}

Figure 6.20: Comparison of highest performance note for each voice type as box and whisker plot and tabular mean values

- Practice vocal range

Mean values for the practice vocal range (Figure 6.21) which is sometimes called the physiological frequency range, were not significantly different between sopranos and mezzo-sopranos.

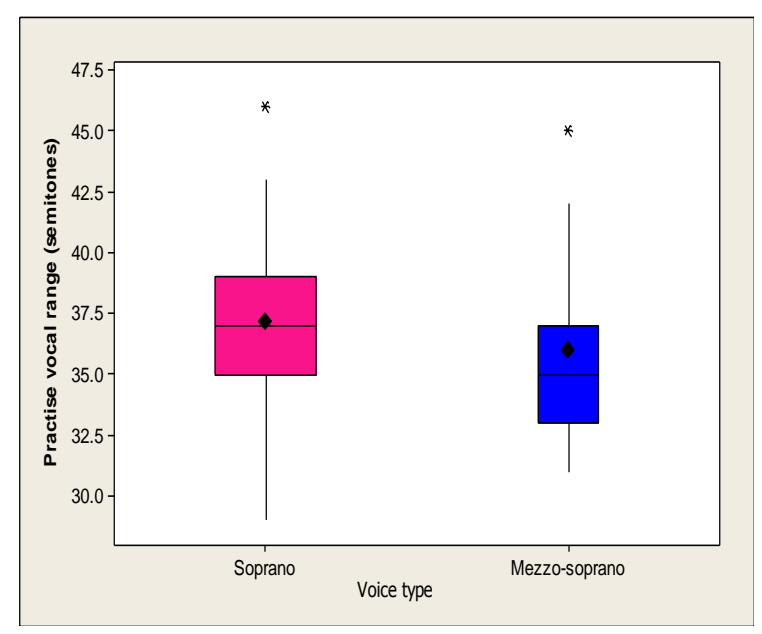

\begin{tabular}{|c|c|c|}
\hline $\begin{array}{c}\text { Voice } \\
\text { Type }\end{array}$ & $\mathbf{n}$ & $\begin{array}{c}\text { Mean } \\
\text { practice } \\
\text { vocal range } \\
\text { (semitones) }\end{array}$ \\
\hline All & 43 & $37 \pm 3.7$ \\
\hline Soprano & 32 & $37 \pm 3.6$ \\
\hline $\begin{array}{c}\text { Mezzo- } \\
\text { soprano }\end{array}$ & 11 & $36 \pm 4.2$ \\
\hline \multicolumn{2}{|l|}{ Significance $(\mathrm{P})$} & 0.417 \\
\hline
\end{tabular}

Figure 6.21: Comparison of practice vocal range for each voice type as box and whisker plot and tabular mean values 
- Results of re-tested measurements

To assess measurement reliability, 14 of the 43 participants were remeasured for neck circumference and length, speaking fundamental frequency, cricoid and thyroid cartilage height and the anterior cricothyroid space. A summary of the re-tested parameters are shown in Table 8.

Table 8: Re-tested parameters showing differences in the mean, standard deviation and standard error of the mean for the selected measurements.

\begin{tabular}{|c|c|c|c|c|c|}
\hline Parameter measured & Reading & $\mathbf{n}$ & Mean & S.D & S.E \\
\hline Neck circumference (cm) & First & 14 & 34.4 & 4.1 & 1.1 \\
\hline \multirow[t]{2}{*}{ Neck circumference $(\mathrm{cm})$} & Second & 14 & 34.2 & 4.2 & 1.1 \\
\hline & Difference & 14 & 0.2 & 0.1 & 0.0 \\
\hline Neck length (cm) & First & 14 & 11.9 & 1.5 & 0.4 \\
\hline \multirow[t]{2}{*}{ Neck length $(\mathrm{cm})$} & Second & 14 & 11.8 & 1.4 & 0.4 \\
\hline & Difference & 14 & 0.1 & 0.1 & 0.0 \\
\hline Cricoid cartilage (mm) & First & 14 & 5.7 & 0.8 & 0.2 \\
\hline \multirow[t]{2}{*}{ Cricoid cartilage (mm) } & Second & 14 & 5.5 & 0.8 & 0.2 \\
\hline & Difference & 14 & 0.2 & 0.0 & 0.0 \\
\hline Cricothyroid space (mm) & First & 14 & 10.4 & 1.6 & 0.4 \\
\hline \multirow[t]{2}{*}{ Cricothyroid space (mm) } & Second & 14 & 10.7 & 1.8 & 0.5 \\
\hline & Difference & 14 & 0.3 & 0.2 & 0.1 \\
\hline Thyroid cartilage $(\mathrm{mm})$ & First & 14 & 12.7 & 1.7 & 0.5 \\
\hline \multirow[t]{2}{*}{ Thyroid cartilage (mm) } & Second & 14 & 12.4 & 1.7 & 0.5 \\
\hline & Difference & 14 & 0.3 & 0.0 & 0.0 \\
\hline $\begin{array}{l}\text { Speaking fundamental frequency } \\
\qquad(\mathrm{Hz})\end{array}$ & First & 14 & 196.9 & 29.1 & 7.8 \\
\hline \multirow[t]{2}{*}{$\begin{array}{l}\text { Speaking fundamental frequency } \\
\qquad(\mathrm{Hz})\end{array}$} & Second & 14 & 196.7 & 28.7 & 7.7 \\
\hline & Difference & 14 & 0.2 & 0.4 & 0.1 \\
\hline
\end{tabular}


Repeated neck measurements made by the same examiner on different occasions were identical in three of 28 readings, within $0.5 \mathrm{~cm}$ in another 21 , and 0.6 to $1.0 \mathrm{~cm}$ different in four. For SFF, three out of 14 values were exactly the same, nine less than $5 \mathrm{~Hz}$ different and two readings were between 6 and $8 \mathrm{~Hz}$ different (Appendix K).

Results from repeated measurements of the larynx (cricoid cartilage height, thyroid cartilage height and anterior cricothyroid space) made by the same sonographer on different occasions were not significantly different; identical values were obtained for eight measurements, 28 were within 0.5 $\mathrm{mm}$, five within 0.6 to $1.0 \mathrm{~mm}$ and only one laryngeal measurement differed by more than $1 \mathrm{~mm}$ (Appendix K).

\subsection{Correlations}

Performance vocal range showed a strong correlation with practice vocal range $(r=0.833 ; P=<0.0005)$ (Appendix $L)$. Performance vocal range rather than practice vocal range was used in subsequent analyses because performance vocal range is the range used by singers for musical demonstration.

\subsubsection{CT space correlations}

- Anthropometric indices

Overall, there was a weak positive correlation between the height of the CT space and body height $(r=0.361 ; P=0.017)$ and neck length $(r=0.408$; $\mathrm{P}=0.007)$. When analysed according to voice type, there was a moderate correlation between the CT space and body height amongst mezzosopranos ( $r=0.645 ; P=0.032)$ but no correlation in sopranos $(r=0.220$; $P=0.226$ ). Likewise, there was a weak positive correlation between $C T$ space and neck length in sopranos $(r=0.421 ; P=0.016)$, but not in mezzosopranos ( $r=-0.060 ; P=0.862$ ). (Appendix $M$ contains correlations for all data, whilst Appendix $\mathrm{N}$ shows the correlations for sopranos and Appendix $O$ the correlations for mezzo-sopranos) 
Age and general anthropometric indices such as body weight, BMI, and neck circumference did not show any significant correlation with CT space distance (Appendix M).

- Laryngeal cartilage dimensions

The height of the cricoid cartilage in the midline showed a weak but significant correlation with the CT space distance $(r=0.397 ; P=0.008)$, whilst the combined length of all the laryngeal cartilages in the midline (thyroid and cricoid cartilage heights and CT space) was more strongly correlated with the CT space distance $(r=0.718 ; P<0.0005)$. Thyroid cartilage height showed no significant correlation with CT space (Appendix M).

- Performance and practice ranges

Neither performance $(r=0.176 ; P=0.259)$ nor practice range $(r=0.103$; $\mathrm{P}=0.512)$ showed a correlation with CT space.

- Speaking fundamental frequency

There was a weak but significant negative correlation between speaking fundamental frequency and CT space (Figure 6.22).

In Figure 6.22, it can be seen that the two participants with the lowest SFF of $152 \mathrm{~Hz}$ had CT space measurements of $10.8 \mathrm{~mm}$ and $11.1 \mathrm{~mm}$, respectively. The participant with the highest SFF of $243 \mathrm{~Hz}$ had a CT space of $9.4 \mathrm{~mm}$. The two participants with the smallest CT space of 7.3 mm had SFF of 207 and $240 \mathrm{~Hz}$, whilst the individual with the largest CT space of $14.0 \mathrm{~mm}$ had a SFF of $163 \mathrm{~Hz}$. 


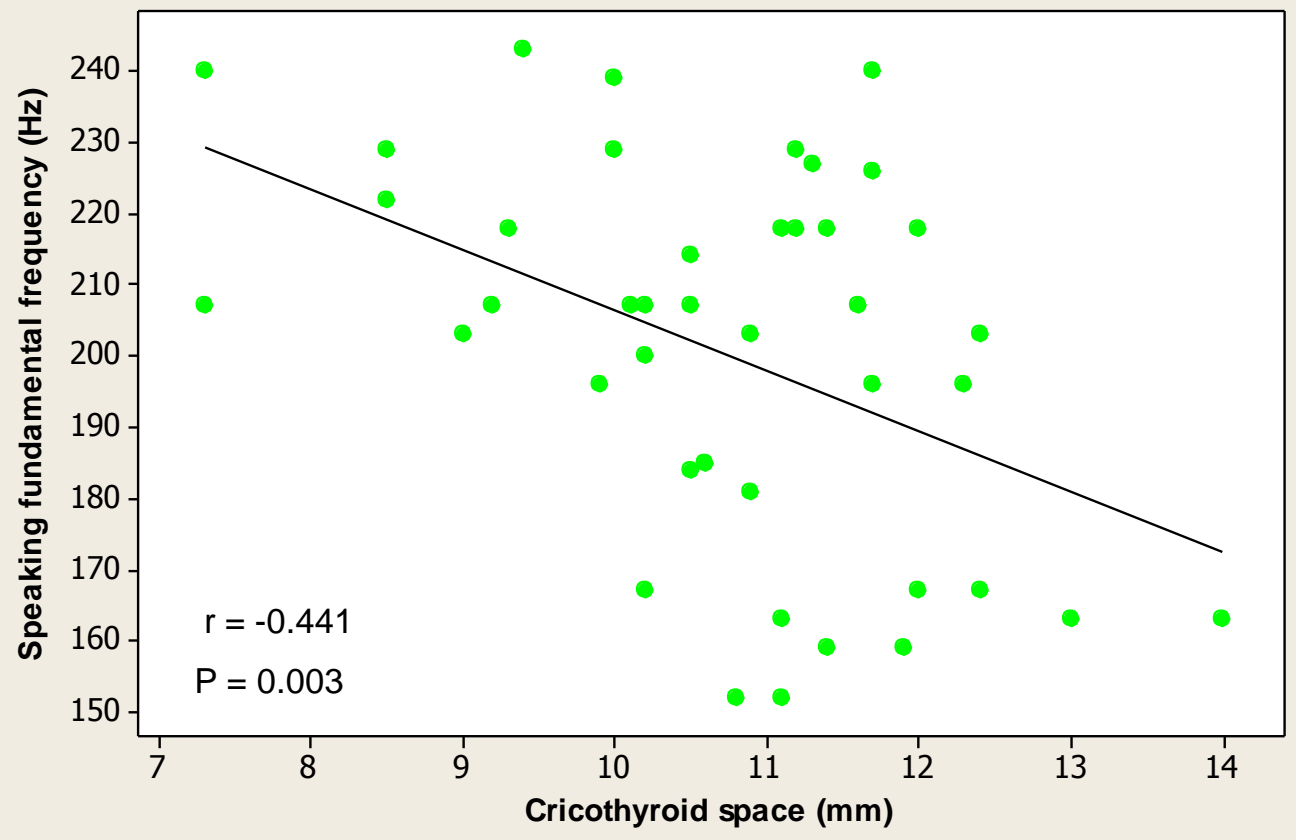

Figure 6.22: Relationship between cricothyroid space and speaking fundamental frequency

- CT space and lowest performance note correlation

There was a weak but significant negative correlation between the lowest performance note and the CT space (Figure 6.23).

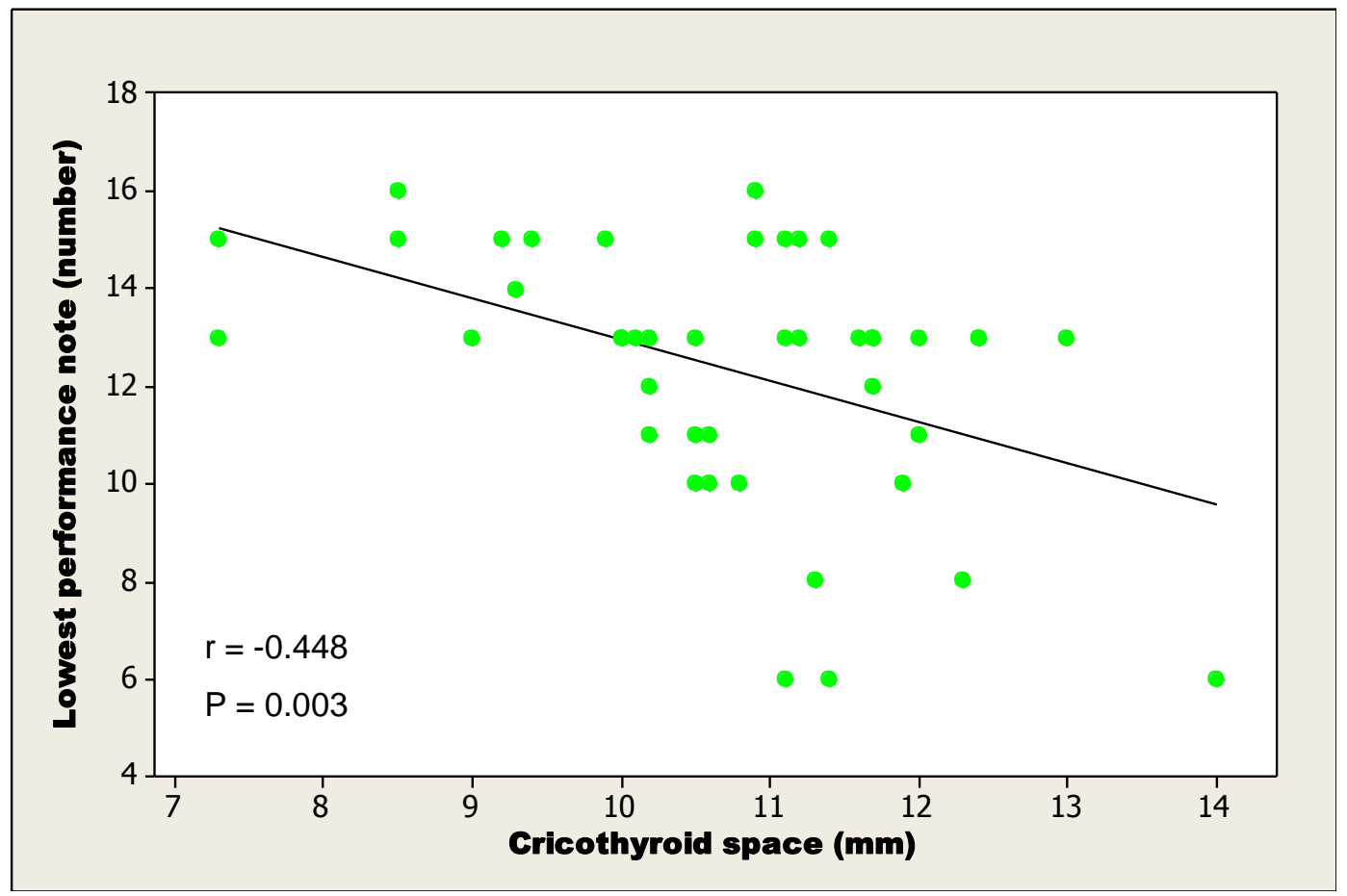

Figure 6.23: Relationship of the cricothyroid space to the lowest performance note (G3 is note number 13) 
This implies that the smaller the CT space the higher the achievable lowest performance note. Further analysis showed that this was evident only in soprano participants (Figure 6.24) and not in mezzo-sopranos ( $r=-$ $0.107 ; P=0.754)$.

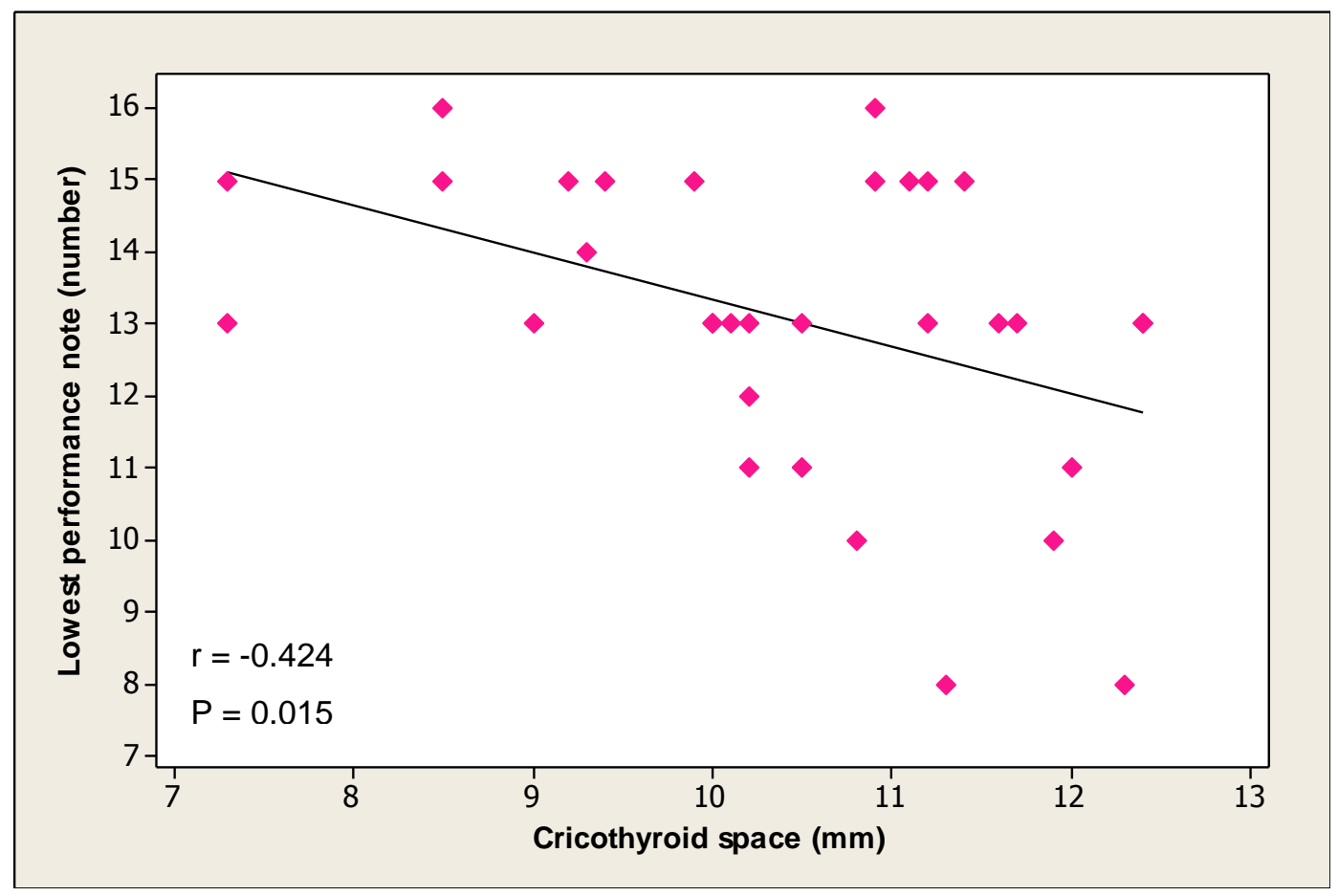

Figure 6.24: Relationship of the cricothyroid space to the lowest performance note in sopranos (G3 is note number 13)

Figure 6.25 shows that all singers with a resting CT space of less than $10.4 \mathrm{~mm}$ were sopranos. There was a tendency for those sopranos with a CT space of less than $10 \mathrm{~mm}$ to have a higher 'lowest performance note' than other sopranos. In contrast, their highest useable note was similar to singers with a resting CT space of more than $10.4 \mathrm{~mm}$ (Figure 6.27). This implies that singers with a small CT space cannot sing low notes and will therefore probably be sopranos. However, a CT space of less than $10 \mathrm{~mm}$ does not predict the type of soprano: lyric, coloratura, spinto etc. 


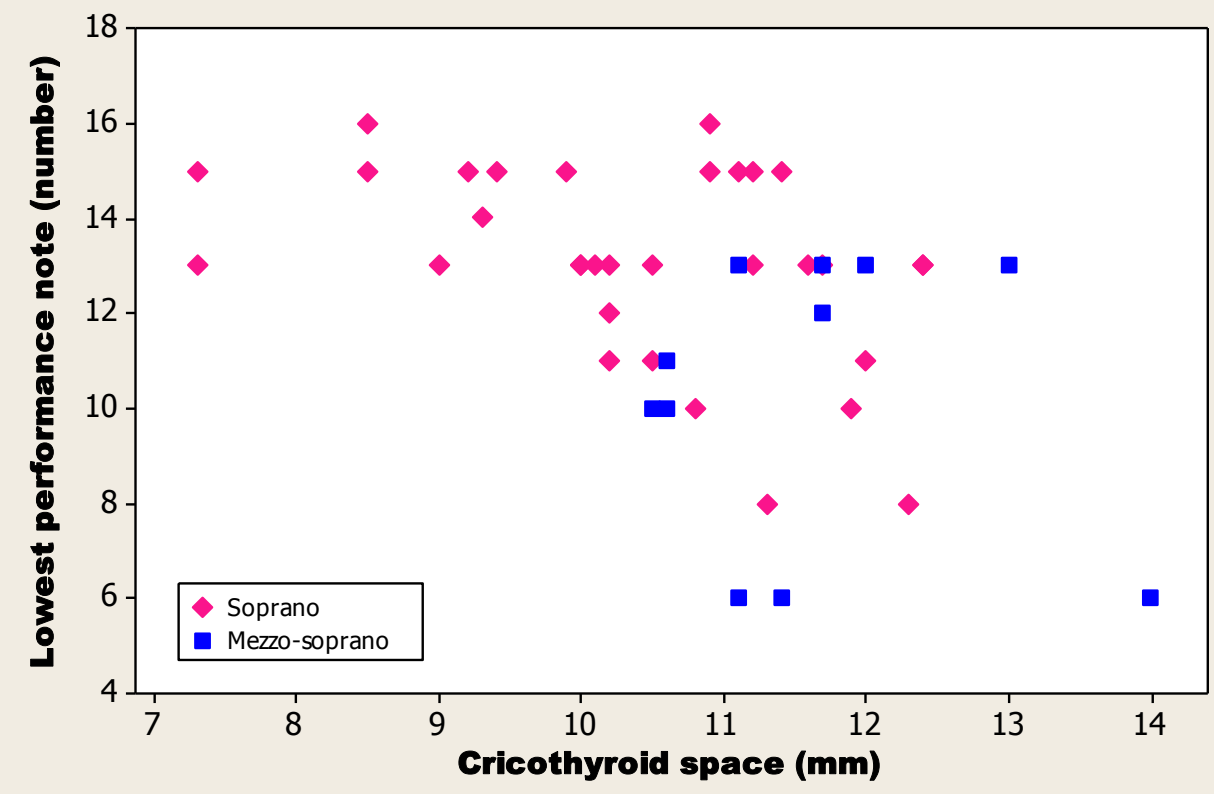

Figure 6.25: Cricothyroid space showing lowest performance notes of sopranos and mezzo-sopranos (G3 is note number 13)

- CT space and highest performance note correlation

There was no significant correlation between the CT space and highest performance note, although there was a statistical trend toward a weak negative correlation (Figure 6.26). This lack of a significant correlation persisted when sopranos $(r=0.016 ; P=0.931)$ and mezzo-sopranos $(r=-$ $0.221 ; P=0.513$ ) were analysed separately. All sopranos with a resting CT space of less than $10.4 \mathrm{~mm}$ had highest performance notes comparable to other sopranos (Figure 6.27). 


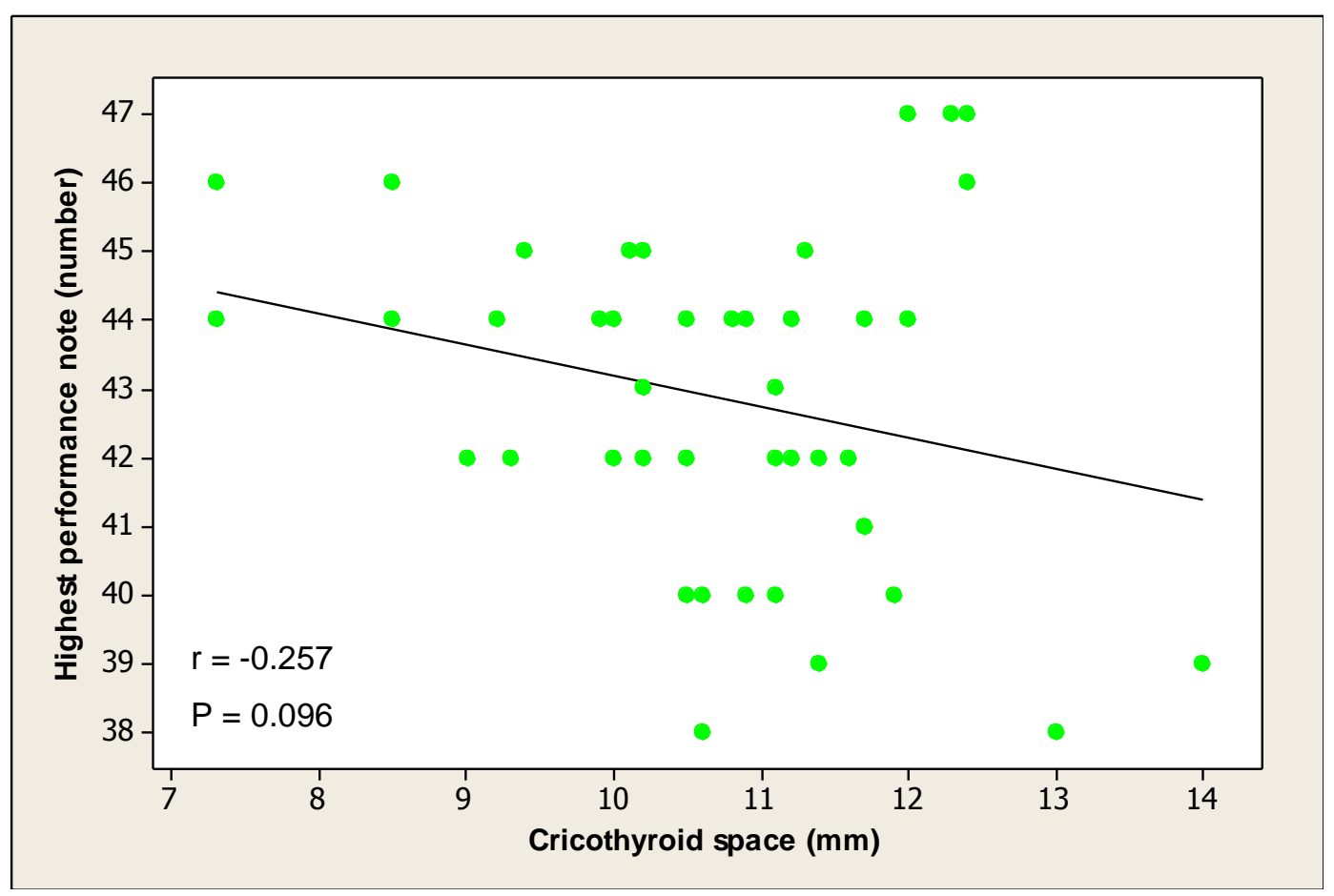

Figure 6.26: Relationship of anterior cricothyroid space with highest performance note (Note number 47 is F6)

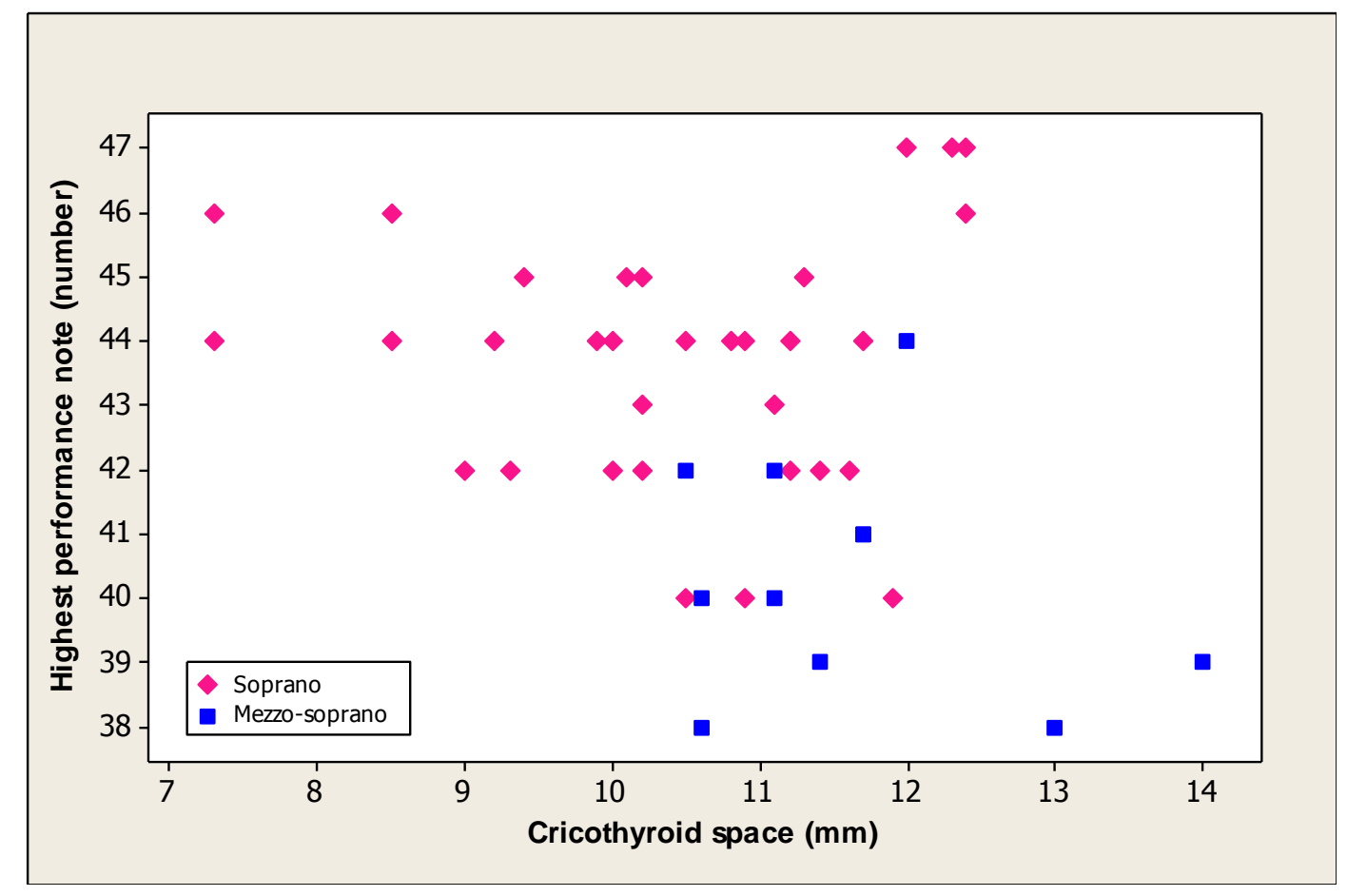

Figure 6.27: Cricothyroid space showing highest performance notes of sopranos and mezzo-sopranos (Note number 47 is F6) 
- CT space and performance vocal range correlation

There was no statistically significant correlation between anterior CT space and performance vocal range in all participants (Figure 6.28) or in sopranos $(r=0.292 ; P=0.105)$ and mezzo-sopranos $(r=-0.029 ; P=0.932)$ when analysed separately.

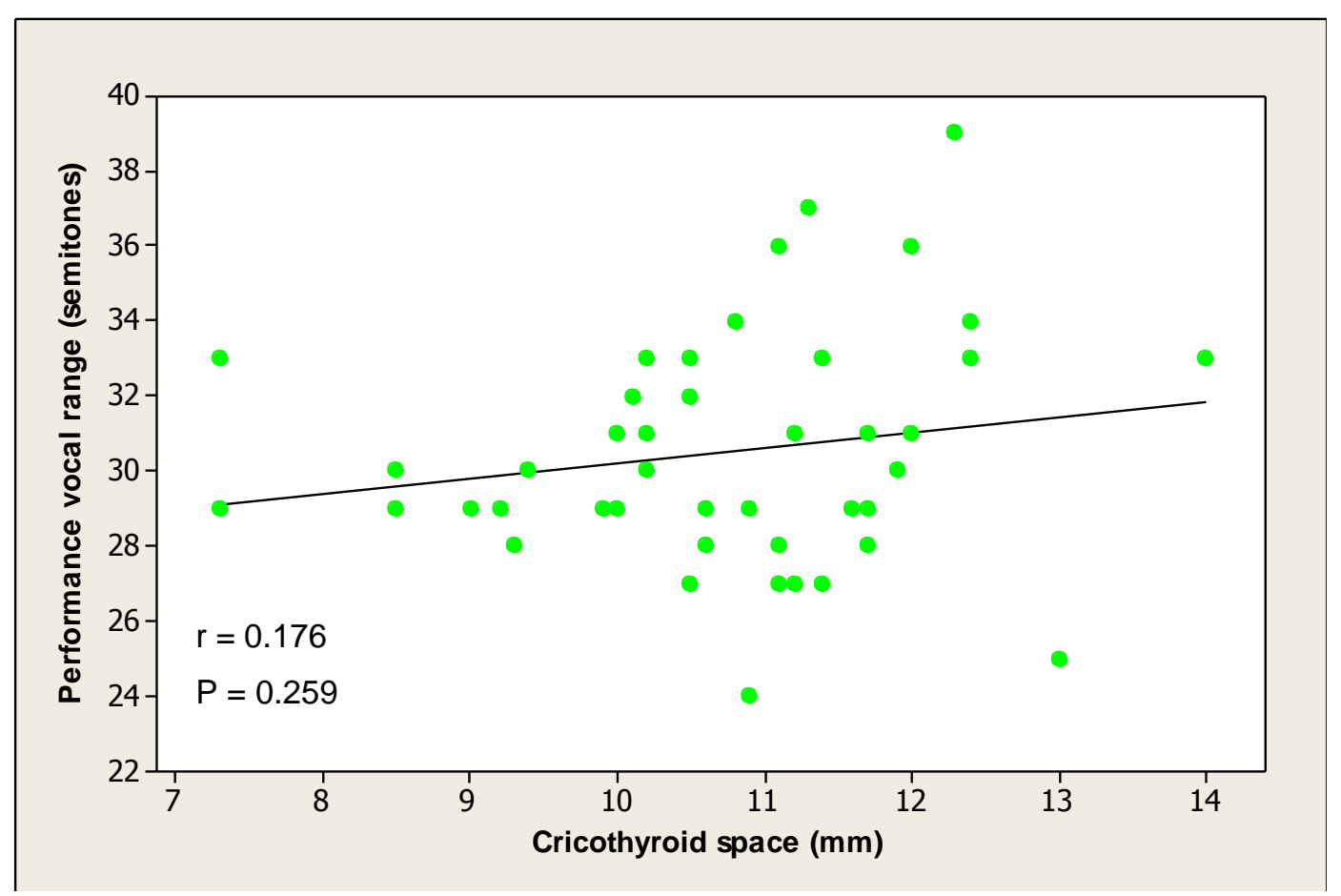

Figure 6.28: Relationship of anterior cricothyroid space with performance vocal range

Looking at Figure 6.28, there were two participants, both sopranos, with the smallest CT space of $7.3 \mathrm{~mm}$, whilst the largest CT space of $14.0 \mathrm{~mm}$ was a mezzo-soprano. The participant with the largest anterior CT space and one of the sopranos (soubrette) with the smallest space both had the same vocal range of 33 semitones. There were also four other participants (one mezzo-soprano and three sopranos) who had a vocal range of 33 semitones but with varying dimensions of their CT space (a soubrette soprano with a space of $10.2 \mathrm{~mm}$, a heavy lyric soprano with a space of $10.5 \mathrm{~mm}$, a mezzo-soprano with a space of $11.4 \mathrm{~mm}$ and a spinto soprano with a space of $12.4 \mathrm{~mm}$ ). The other soprano (spinto) with smallest anterior CT space had a vocal range of 29 semitones. 
The participants with the largest (39 semitones) and smallest (24 semitones) vocal ranges were both light lyric sopranos with CT space dimensions of $12.3 \mathrm{~mm}$ and $10.9 \mathrm{~mm}$, respectively. (Appendix $\mathrm{P}$ contains a summary of the raw data).

\subsubsection{Speaking fundamental frequency correlations}

There were weak but significant negative correlations of SFF with body weight $(r=-0.332 ; P=0.030)$ and the total length of the laryngeal cartilages $(r=-0.363 ; P=0.017)$. In addition, the lowest performance note $(r=0.517$; $\mathrm{P}=<0.0005)$ and highest performance note $(r=0.358 ; \mathrm{P}=0.018)$ showed significant weak positive correlations with the SFF.

\subsubsection{Other performance vocal range correlations}

There were weak significant correlations between performance vocal range and neck length $(r=0.372 ; P=0.014)$, thyroid cartilage length $(r=0.403 ; P=0.007)$ and the total length of the laryngeal cartilages $(r=0.332$; $\mathrm{P}=0.029$ ).

Overall, there was a moderate negative correlation between performance vocal range and lowest performance note $(r=-0.661 ; P<0.0005)$ and $a$ weak positive correlation between performance vocal range and highest performance note $(r=0.582 ; P<0.0005)$. With regards to voice types, sopranos had a moderate negative correlation between performance vocal range and lowest performance note $(r=-0.794 ; P=<0.0005)$ and $a$ moderate positive correlation between performance vocal range and highest performance note $(r=0.760 ; P<0.0005)$. Mezzo-sopranos had a stronger negative correlation between performance vocal range and lowest performance note $(r=-0.822 ; P=0.002)$ but no significant correlation between performance vocal range and highest performance note $(r=0.413$; $\mathrm{P}=0.207)$. 
Performance vocal range did not decrease significantly over the age range of the study sample $(r=-0.171 ; P=0.273)$ and there was no significant reduction in lowest $(r=-0.034 ; P=0.828)$ or highest performance note $(r=-$ $0.259 ; P=0.094$ ) with age (Appendix $Q$ has summary of data comparing age to performance vocal range). However, one of the two oldest participants (63 years) did have the smallest vocal range of only 23 semitones (one note under 2 octaves). She reported that her highest performance note had indeed decreased in the last few years but her lowest performance note had not altered. The other 63 year old participant had a vocal range of 29 semitones which was comparable with younger singers. The youngest participant (18 years) had a vocal range of 31 semitones which was the same as a 60 year old. The participant with the largest performance range of 39 semitones (just over 3 octaves) was aged 35 years.

There was no significant correlation between performance vocal range and speaking fundamental frequency $(r=-0.155 ; P<0.0005)$. Also, there were no significant correlations between performance vocal range and height, weight, BMI, neck circumference and the height of the cricoid cartilage (all $r$ and $P$ values are noted in Appendix $\mathrm{M}, \mathrm{N}$ and $\mathrm{O}$ ).

\subsubsection{Other anthropometric correlations}

There was a weak significant positive correlation between stature and cricoid cartilage height $(r=0.346 ; P=0.023)$, but not between stature and midline thyroid cartilage height $(r=0.229 ; P=0.140)$. However, there was a weak positive correlation between stature and total length of the laryngeal cartilages ( $r=0.405 ; P=0.007)$, evident amongst mezzo-sopranos $(r=0.689$; $\mathrm{P}=0.019)$ but not sopranos $(r=0.237 ; \mathrm{P}=0.139)$.

No correlation was found between stature and neck circumference $(r=0.203 ; P=0.192)$. 


\subsubsection{Correlations between re-tested parameters}

Overall, there were strong positive correlations between the first and second measurements on all re-tested parameters.
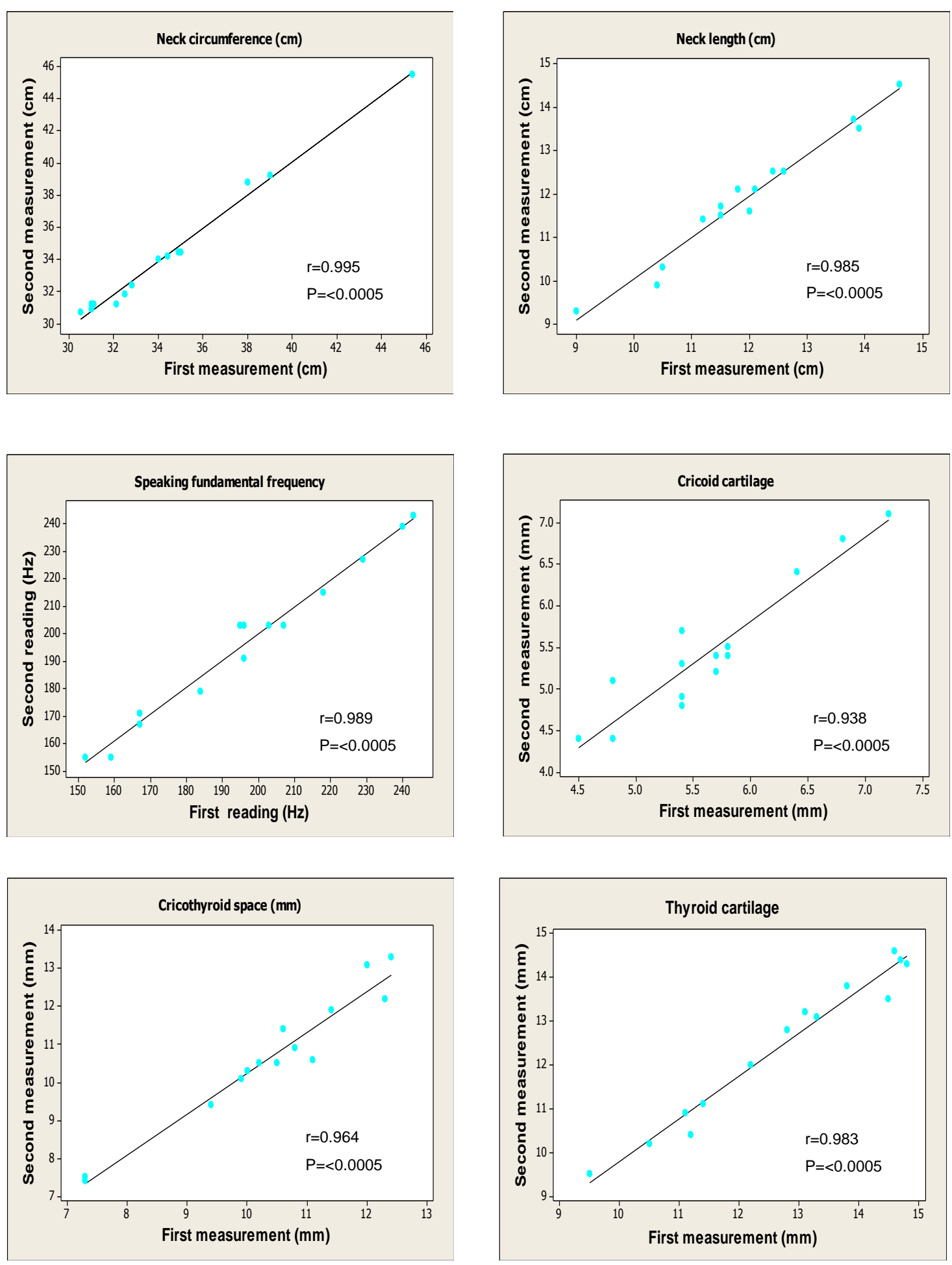

Figure 6.29: Relationship between first and second measurements on selected parameters 


\section{Discussion}

\subsection{Main findings}

The main finding in this study was that mezzo-sopranos have a significantly greater mean CT space than sopranos. As expected, mezzosopranos also had significantly lower mean values of lowest performance note, highest performance note, and speaking fundamental frequency $(\mathrm{Hz})$ than sopranos (Table 9). Mezzo-sopranos tended to be taller with a longer neck (although the latter was not statistically significant).

This research suggests that there may be a relationship between CT space and the lowest note a female can sing (refer back to Figure 6.23). This was most apparent in the correlation of a small CT space with a higher 'lowest performance note'. It appears that a smaller CT space plays a major part in determining how low one can sing, but does not limit how high one can sing. 
Table 9: Summary of selected parameters according to voice type expressed as means and standard deviations

\begin{tabular}{|c|c|c|c|}
\hline Parameter & $\begin{array}{l}\text { Sopranos } \\
\qquad n=32\end{array}$ & $\begin{array}{l}\text { Mezzo-sopranos } \\
\qquad n=11\end{array}$ & $P$ value \\
\hline $\begin{array}{l}\text { Cricothyroid space } \\
\qquad(\mathrm{mm})\end{array}$ & $10.4 \pm 1.4$ & $11.6 \pm 1.1$ & $0.007^{*}$ \\
\hline $\begin{array}{l}\text { Lowest performance } \\
\text { note (pitch) }\end{array}$ & $\mathrm{G} 3 \pm 2.1$ & E3 \pm 3.0 & $0.013^{*}$ \\
\hline $\begin{array}{c}\text { Highest } \\
\text { performance note } \\
\text { (pitch) }\end{array}$ & $\mathrm{D} 6 \pm 2.0$ & $A \# 5 \pm 1.9$ & $<0.0005^{*}$ \\
\hline $\mathrm{SFF}(\mathrm{Hz})$ & $207 \pm 23.1$ & $180 \pm 26.3$ & $0.008^{*}$ \\
\hline $\begin{array}{l}\text { Performance vocal } \\
\text { range (semitones) }\end{array}$ & $31 \pm 3.2$ & $30.1 \pm 3.2$ & 0.619 \\
\hline Body height $(\mathrm{cm})$ & $165.0 \pm 6.6$ & $168.2 \pm 6.8$ & 0.175 \\
\hline Neck length $(\mathrm{cm})$ & $11.9 \pm 1.7$ & $12.7 \pm 1.0$ & 0.061 \\
\hline
\end{tabular}

${ }^{*}$ Statistical significance $\mathrm{P}<0.05$

There was no significant correlation between CT space and vocal range (the distance between the lowest and highest performance notes) in either sopranos or mezzo-sopranos. This suggests that the CT space does not determine performance vocal range. 


\subsection{Comparison with previous studies}

\subsubsection{Anthropometric indices}

The present study found a weak positive correlation between stature and cricoid cartilage height, but not between stature and midline thyroid cartilage height. This differs from a previous study where Jain and Dhall (2008) found statistically significant positive correlations between stature and the height of the thyroid lamina (male $r=0.696$, female $r=0.461$ ) height of the cricoid arch (male $r=0.719$, female $r=0.511$ ) and the transverse diameter of the cricoid cartilage (male $r=0.576$, female 0.752 ). However, Ajmani et al. (1980) and Longia (1990) found no such correlations.

Although not statistically significant, a relationship between stature and voice type was noted in this study. Mean height for sopranos was 164.9 $\mathrm{cm}$ and for mezzo-sopranos $168.2 \mathrm{~cm}$. These values are lower than those obtained by Larsson and Hertegård (2008) who recorded an average soprano height $(n=9)$ of $169(162-175) \mathrm{cm}$ and average mezzo-soprano height $(n=5)$ of $174(170-177) \mathrm{cm}$. Roers (2005), on the other hand, reported similar results to this study with an average soprano $(n=97)$ height of $166.1 \mathrm{~cm}$ and average mezzo-soprano ( $n=56)$ height of 167.9 $\mathrm{cm}$. All these results agree that on average mezzo-sopranos are taller than sopranos. This concurs with Roers et al. (2007) who showed that the taller the singer in general, the lower the voice type.

Roers (2005) found the average weight of sopranos ( $n=97$ ) was $59.0 \mathrm{~kg}$ and mezzo-sopranos ( $n=54) 60.0 \mathrm{~kg}$; this compares with $72.0 \mathrm{~kg}$ and 76.1 $\mathrm{kg}$, respectively in the current study, which may reflect population trends in weight. With respect to BMI, Roers (2005) found that they were similar between voice types: sopranos ( $\mathrm{n}=97) 21.1 \mathrm{~kg} / \mathrm{m}^{2}$ and mezzo-sopranos $(n=54) 21.2 \mathrm{~kg} / \mathrm{m}^{2}$. The present study concurs with this, as sopranos had an average BMl of $26.8(19.5-38.5) \mathrm{kg} / \mathrm{m}^{2}$ while mezzo-sopranos were $26.9(19.3-44.6) \mathrm{kg} / \mathrm{m}^{2}$. 
In conclusion, there are no consistent correlations between voice type and anthropometric indices.

\subsubsection{Neck measurements}

There is a paucity of data comparing neck dimensions with SFF or voice category although mean values from other studies (Table 10) suggest no strong relationship between these variables.

Table 10: Comparison of neck dimensions

\begin{tabular}{|c|c|c|c|c|c|}
\hline & & \multicolumn{2}{|c|}{ Soprano } & \multicolumn{2}{c|}{ Mezzo-soprano } \\
\hline Author & Year & $\mathbf{n}$ & $\begin{array}{c}\text { Neck diameter } \\
\text { (cm) }\end{array}$ & $\mathbf{n}$ & $\begin{array}{c}\text { Neck diameter } \\
\text { (cm) }\end{array}$ \\
\hline Roers & 2005 & 86 & 32.1 & 49 & 32.5 \\
\hline $\begin{array}{c}\text { Larsson and } \\
\text { Hertegård }\end{array}$ & 2008 & 9 & $35(31-43)$ & 5 & $35(33-37)$ \\
\hline This study & 2012 & 31 & $34.0(30.0-45.5)$ & 12 & $34.0(30.5-39.8)$ \\
\hline
\end{tabular}

Larsson and Hertegård (2008) found a moderate positive correlation between neck circumference and height $(r=0.60)$ but no such correlation was evident in this study. 


\subsubsection{CT space and laryngeal cartilages}

From this study the average female thyroid cartilage height of $12.1 \pm 2.0$ $\mathrm{mm}$ was lower than values reported in previous studies (Table 11). These previous reports recorded "anterior thyroid height", but it is questionable as to whether they were actually measuring the height of the thyroid lamina rather than the height of the thyroid cartilage in the midline. (The height of the thyroid cartilage in the midline will be less because of the presence of the thyroid notch).

The average cricoid cartilage height in the present study was $5.7 \pm 0.7 \mathrm{~mm}$ in adult females. This value is similar to results reported by Tayama (2001) and Jain and Dhall (2008) who recorded $5.4 \pm 0.01 \mathrm{~mm}$ and $5.6 \pm 1.0 \mathrm{~mm}$, respectively (Table 11 ).

The mean cricothyroid space distance in females obtained in this study was $10.7 \pm 1.4 \mathrm{~mm}$ which was higher than previous reports of this measurement in cadavers (Table 10). Given that the mean total height of the thyroid and cricoid cartilages together with the CT space was $28.5 \pm$ $2.9 \mathrm{~mm}$ in this study, the results were similar to those of Tayama et al. (2001) who recorded a value of $26.75 \mathrm{~mm}$ (standard deviation not stated). However, since the present study uniquely used ultrasound to systematically measure laryngeal dimensions in live subjects, differences in methodology could account for some of the differences observed between the current results and those obtained from cadavers in previous studies.

The study sample consisted of mainly European New Zealanders with a small proportion of Māori, so it is unlikely that ethnicity would account for any major differences with previous studies. Furthermore, given the vagueness and complexity of the ethnicity in New Zealand (see NZ Statistics definitions) no conclusions can be drawn about this aspect of the study. 
Table 11: Comparison of female thyroid and cricoid cartilage measurements and cricothyroid space with previous studies

\begin{tabular}{|c|c|c|c|c|c|c|c|}
\hline Author & Ethnicity & $\mathbf{n}$ & $\begin{array}{l}\text { Study } \\
\text { sample }\end{array}$ & $\begin{array}{c}\text { Anterior thyroid vertical } \\
\text { height in midline }(\mathrm{mm}) \\
\pm \mathrm{S} . \mathrm{D}\end{array}$ & $\begin{array}{c}\text { Cricoid arch vertical height } \\
\text { in midline }(\mathrm{mm}) \\
\pm \text { S.D }\end{array}$ & $\begin{array}{l}\text { Cricothyroid } \\
\text { vertical space } \\
(\mathrm{mm}) \\
\pm \text { S.D }\end{array}$ & $\begin{array}{c}\text { Total height of } \\
\text { cartilages and } \\
\text { space } \\
(\mathrm{mm}) \pm \text { S.D }\end{array}$ \\
\hline $\begin{array}{c}\text { Chievitz } \\
(1882)\end{array}$ & European & 135 & Cadavers & 15.8 & 6.8 & & \\
\hline $\begin{array}{l}\text { Maue and Dickson } \\
(1971)\end{array}$ & North American & 10 & Cadavers & $26.0^{7}$ & $3.1^{7}$ & & \\
\hline $\begin{array}{l}\text { Ajmani } \\
\text { (1990) }\end{array}$ & Nigerian & 12 & Cadavers & $17.3 \pm 6.6$ & $7.5 \pm 4.3$ & & \\
\hline $\begin{array}{l}\text { Eckel et al. } \\
\text { (1994) }\end{array}$ & German & 25 & Cadavers & $15.8 \pm 1.1$ & $6.2 \pm 1.1$ & & \\
\hline Tayama (2001) & Japanese & 3 & Cadavers & 13.6 & $5.4 \pm 0.01$ & 8.6 & 26.8 \\
\hline $\begin{array}{l}\text { Jain and Dhall } \\
\text { (2008) }\end{array}$ & Indian & 20 & Cadavers & $13.4 \pm 3.2$ & $5.6 \pm 1.0$ & & \\
\hline $\begin{array}{l}\text { Hammer et al., } \\
(2010) \\
\text { Windisch et al., } \\
(2010)\end{array}$ & Austrian & 25 & Cadavers & & & $6.6 \pm 1.8$ & \\
\hline This study & $\begin{array}{c}\text { European and } \\
\text { Māori }\end{array}$ & 43 & $\begin{array}{l}\text { Living } \\
\text { Ultrasound }\end{array}$ & $12.1 \pm 2.0$ & $5.7 \pm 0.7$ & $10.7 \pm 1.4$ & $28.5 \pm 2.9$ \\
\hline
\end{tabular}

\footnotetext{
${ }^{7}$ Questionable data
} 


\subsubsection{Speaking Fundamental Frequency}

In the literature, the normal mean SFF for premenopausal women is 224 $\mathrm{Hz}$ (range 180-250 Hz) and for postmenopausal women $200 \mathrm{~Hz}$ (range 160-230). The mean SFF obtained in this study was $200 \mathrm{~Hz}$ (range 152$243 \mathrm{~Hz}$ ) which is reasonably similar. Menopausal status was not recorded in this study but 24 women were less than 40 years and therefore this is likely to represent a conservative estimate of the proportion who were premenopausal; their SFF was $202 \mathrm{~Hz}$, compared with over 40 year olds whose SFF was $199 \mathrm{~Hz}$.

A comparison of SFFs of sopranos and mezzo-sopranos in this study with published data is shown in Table 12. Mean values for sopranos and mezzo-sopranos in this study were within the range of values reported in previous studies. Lower values in mezzo-sopranos compared to sopranos is also consistent with previous reports.

Table 12: Comparison of average SFF values in voice types (ranges in parentheses)

\begin{tabular}{|c|c|c|c|c|c|}
\hline & & \multicolumn{2}{|c|}{ Soprano } & \multicolumn{2}{c|}{ Mezzo-soprano } \\
\hline Author & Task type & $\mathbf{H z}$ & $\mathbf{n}$ & $\mathbf{H z}$ & $\mathbf{n}$ \\
\hline $\begin{array}{c}\text { Nadoleczny } \\
(1923)\end{array}$ & Reading & 262 & 10 & 230 & 10 \\
\hline $\begin{array}{c}\text { Roers } \\
(2005)\end{array}$ & $\begin{array}{c}\text { Reading and } \\
\text { spontaneous } \\
\text { speech }\end{array}$ & 233.5 & 94 & 220 & 17 \\
\hline $\begin{array}{c}\text { Larsson and } \\
\text { Hertegård } \\
(2008)\end{array}$ & Reading & $\begin{array}{c}189 \\
(160-247)\end{array}$ & 7 & $\begin{array}{c}177 \\
(153-205)\end{array}$ & 7 \\
\hline This study & Reading & $\begin{array}{c}207 \\
(152-243)\end{array}$ & 31 & $\begin{array}{c}180 \\
(152-240)\end{array}$ & 12 \\
\hline
\end{tabular}

${ }^{8}$ Both reading and spontaneous speech tasks were used to determine SFF. Both tasks correlated well with each other with no significant difference for values found between task type. 
A lower SFF in older women was not apparent in this study. One of the oldest participants (age 63 years) had the second highest SFF of $240 \mathrm{~Hz}$, a value that was considerably higher than the practice mean and that recorded in the literature. Conversely, the youngest participant, who was 18 years old, had a SFF of $167 \mathrm{~Hz}$, which is substantially below the practice mean of the study sample and the mean reported in previous studies. These study results appear consistent with the findings of Hollien and Shipp (1972), Brown Jr et al. (1991) and Morris et al. (1995) who noted that age related changes in SFF seemed to be less prominent or even absent in trained singers.

Debruyne et al. (2002) studied SFF in 30 female monozygotic (MZ) twins and 30 dizygotic (DZ) twins using a reading task. Values were more congruent in the $M Z$ twins, which the authors concluded was compatible with a genetic basis for SFF. In the present study there was one pair of DZ twins and a mother and daughter. The SFF results from the pair of DZ twins (both sopranos) were $222 \mathrm{~Hz}$ and $214 \mathrm{~Hz}$, whilst the SFFs from mother and daughter (both sopranos) were 243 and $239 \mathrm{~Hz}$ respectively. No conclusions can be drawn from these single cases but the results are at least compatible with Debruyne's suggestion of a genetic predisposition.

Numerous studies have been conducted on the effects of ethnicity on SFF. It has been suggested that physical differences between speakers of different ethnic origins may result in acoustic differences in vocal characteristics including SFF (Hollien \& Malcik, 1962; Hudson \& Holbrook, 1982; Xue \& Mueller, 1996). The numbers of participants from different ethnic backgrounds in this study are too small to draw any meaningful conclusions about ethnicity and SFF.

Some studies have suggested a correlation between SFF and physical characteristics, in particular a negative correlation between height and/or weight (Laver \& Trudgill, 1979). Larsson and Hertegård (2008) found a strong negative correlation $(r=-0.72)$ between SFF and body height in their study of 27 singers (14 females and 13 males). The findings in the present 
study concur with their results in that shorter individuals tend to have a higher SFF and taller singers a lower SFF. Evans (2006) found weight was significantly negatively correlated with SFF $(r=-0.34)$ in a study on 50 men. Although the current study was undertaken on females, a similar significant weak negative correlation of SFF with weight was found $(r=-$ 0.33).

\subsubsection{Vocal range}

Universally accepted performance vocal ranges of female sopranos and mezzo-sopranos (and Fach types) compared to those in this study are shown in Tables 13 and 14. Average ranges for sopranos and mezzosopranos (and Fach types) in the current study exceeded typical vocal ranges. Just about all vocal categories had a $2 \frac{1}{2}$ octave range. As expected, the mezzo-soprano range was at a lower tessitura than the sopranos, being E3 - A\#5 and G3 - D6, respectively. Both practice ranges were comparable. In a study by Roers (2005), vocal ranges were B3-G\#5 in sopranos $(n=38)$ and A3-F\#5 in mezzo-sopranos $(n=21)$. Whilst the vocal range for mezzo-sopranos in both the Roers study and the present study was comparable to normal, the vocal range for sopranos differed in the Roers study, especially in the highest performance note which was lower than expected.

Table 13: Comparison of average performance vocal ranges between sopranos and mezzo-sopranos

\begin{tabular}{|c|c|c|c|c|}
\hline & \multicolumn{2}{|c|}{$\begin{array}{l}\text { Normal data }{ }^{9} \\
\text { Middle } C=C 4\end{array}$} & \multicolumn{2}{|c|}{$\begin{array}{c}\text { This study } \\
\text { Middle } \mathrm{C}=\mathrm{C4}\end{array}$} \\
\hline Voice Type & $\begin{array}{c}\text { Average } \\
\text { vocal range in } \\
\text { note names }\end{array}$ & $\begin{array}{c}\text { Average } \\
\text { vocal range in } \\
\text { number of } \\
\text { semitones }\end{array}$ & $\begin{array}{c}\text { Average } \\
\text { vocal range in } \\
\text { note names }\end{array}$ & $\begin{array}{c}\text { Average } \\
\text { vocal range in } \\
\text { number of } \\
\text { semitones }\end{array}$ \\
\hline Soprano & $\mathrm{C} 4-\mathrm{C} 6$ & 24 & G3 - D6 & 31 \\
\hline Mezzo-soprano & $\mathrm{A} 3-\mathrm{A} 5$ & 24 & E3 - A\#5 & 30 \\
\hline
\end{tabular}

9 (O'Connor, 2012) 
Table 14: Comparison of average performance vocal ranges between Fach

\begin{tabular}{|c|c|c|c|c|}
\hline & \multicolumn{2}{|c|}{$\begin{array}{l}\text { Normal data }{ }^{10} \\
\text { Middle C= C4 }\end{array}$} & \multicolumn{2}{|c|}{$\begin{array}{c}\text { This study } \\
\text { Middle C= C4 }\end{array}$} \\
\hline Voice Type & $\begin{array}{l}\text { Average } \\
\text { vocal range in } \\
\text { note names } \\
\text { note names }\end{array}$ & $\begin{array}{c}\text { Average } \\
\text { vocal range in } \\
\text { number } \\
\text { semitones }\end{array}$ & $\begin{array}{c}\text { Average } \\
\text { vocal range in } \\
\text { note names }\end{array}$ & $\begin{array}{l}\text { Average } \\
\text { vocal range } \\
\text { in number of } \\
\text { semitones }\end{array}$ \\
\hline Coloratura & $\mathrm{C} 4-\mathrm{F} 6$ & 29 & $\mathrm{~A} 3-\mathrm{E} 6$ & $31(n=4)$ \\
\hline Spinto & $\mathrm{C} 4-\mathrm{C} 6$ & 24 & G3 - C\#6 & $30(n=6)$ \\
\hline Light Lyric Soprano & $\mathrm{C} 4-\mathrm{C} 6$ & 24 & $\mathrm{G} 3-\mathrm{C \# 6}$ & $31(n=10)$ \\
\hline $\begin{array}{c}\text { Heavy Lyric } \\
\text { Soprano }\end{array}$ & C4 - C6 & 24 & F\#3 - D6 & $32(n=5)$ \\
\hline Soubrette & C4 - C6 & 24 & G\#3 - D6 & $31(n=4)$ \\
\hline Dramatic Soprano & B3 - C6 & 25 & G3 - C6 & $29(n=3)$ \\
\hline
\end{tabular}

10 (Suverkrop, 2013) 
Larsson and Hertegård (2008) compared vocal ranges of sopranos and mezzo-sopranos among professional singers. They reported average ranges of 33 and 31 semitones, respectively for sopranos and mezzosopranos. These ranges compare well with 31 and 30 semitones, respectively obtained in this study. Both studies showed that sopranos have a slightly wider vocal range than mezzo-sopranos.

There were no significant age related differences in vocal range, in particular no change in the vocal range, with progressive loss of top voice, expansion of lower voice, and reduction in total voice range with increasing age as described in the literature (Mueller, et al., 1984; Linville, 1987; Sataloff, et al., 1997; Teles-Magalhães, et al., 2000; Verdonck-de Leeuw \& Mahieu, 2004). The performance vocal range did not decrease over the age range of the study sample and there was no reduction in the lowest and highest performance note with age. A 60 year old had the same performance vocal range of 31 semitones as the youngest 18 year old participant. This range was consistent with the mean vocal range in the current study and considerably higher than the mean vocal range recorded in the literature. Apart from one participant who reported that her highest performance note had decreased in the last few years, while her lowest performance note had not altered, no other participants reported a dropping off of highest or lowest performances notes with age. 


\section{Practical Implications}

The dimension of the anterior CT space showed no significant correlation with performance vocal range in this study. It appears that a narrow CT space does not limit how high one can sing but does affect how low one can sing. Participants with the narrowest and widest anterior CT space had similar vocal ranges. The CT space narrows with rising pitch, but this dynamic process does not necessarily mean that it determines vocal range. Other factors must be involved.

The control of fundamental frequency is primarily related to several complex underlying physiological mechanisms, functioning together or separately, which may or may not alter the CT space in the process. The principal biomechanical factor regulating $F_{0}$ is the tension on the vocal folds which can be achieved by lengthening them or making them intrinsically more taut. Both of these activities are controlled by the opposing actions of the CT and TA muscles, each having a different effect on the CT space. At high pitches the $F_{0}$ is primarily regulated by the CT muscle, which lengthens the vocal folds (closing the CT space). Conversely, in low pitches the $F_{0}$ is mainly regulated by the TA muscle which shortens the vocal folds (opening the CT space).

A higher pitch produced by increasing vocal fold tension with concurrent vocal fold lengthening occurs as a result of the CT muscle acting on the CTJ (Colton, 1988; Honda, 2004). However, as mentioned previously, the CT muscle is composed of two parts: pars recta and pars obliqua. These have different direct actions on the CTJ, varying the length and/or tension of the vocal folds, and a different effect on the CT space. The pars recta (functions across all F0) rotates the thyroid cartilage down towards the cricoid cartilage along a vertical axis (Vilkman, et al., 1996) which reduces the CT space and results in increased length and tension in the vocal folds (Hong, et al., 1998). The pars obliqua (mostly used for upper pitches) moves the cricoid cartilage backwards (forward translation of the thyroid cartilage) with simultaneous forward anteroposterior gliding at the CT joint. 
This results in vocal fold lengthening and minimal CT space closure due to the forward horizontal movement.

The second way that a higher pitch can be produced by increasing vocal fold tension occurs without concurrent lengthening of the vocal folds. There are two possibilities for this manifestation. Either, when the vocal folds are elongated to the point where the collagenous fibres cannot yield any further, or when the CT and vocalis reach an isometric point. When these conditions occur, vocal fold tension and stiffness can be increased, but no vocal fold lengthening ensues (Miller, 2000). This increases the $F_{0}$ without CTJ rotation and therefore the CT space remains relatively unchanged.

In summary, there are mechanisms by which vocal pitch can be varied without altering the CT space. The two possible strategies to increase pitch without closing the CT space are: increasing the length of vocal folds (anteroposterior gliding of the CTJ), or increasing vocal fold tension only (Table 15). Of course, pitch can still be increased with the closing of the CT space by rotation of the CTJ with lengthening and tensing of the vocal folds, but the strategies that do not only involve CT space changes are advantageous to singers. 
Table 15: Summary of pitch raising mechanisms and their effect on the CT space

\begin{tabular}{|c|c|c|c|c|}
\hline $\begin{array}{c}\text { Vocal folds } \\
\text { Increased tension }\end{array}$ & CTJ & Muscle used & $\begin{array}{c}\text { CT } \\
\text { space }\end{array}$ & $\begin{array}{c}\text { Frequency } \\
\text { range }\end{array}$ \\
\hline $\begin{array}{c}\text { Lengthen } \\
\text { Increased tension }\end{array}$ & $\begin{array}{c}\text { Anteroposterior } \\
\text { gliding }\end{array}$ & $\begin{array}{c}\text { CT } \\
\text { Pars recta }\end{array}$ & Closes & All frequencies \\
\hline $\begin{array}{c}\text { No lengthening } \\
\text { Increased tension }\end{array}$ & $\begin{array}{c}\text { No rotation or } \\
\text { anteroposterior } \\
\text { gliding }\end{array}$ & $\begin{array}{c}\text { CT and vocalis } \\
\text { (Isometric) } \\
\text { closure }\end{array}$ & $\begin{array}{c}\text { No } \\
\text { closure } \\
\text { frequencies }\end{array}$ & All \\
\hline $\begin{array}{c}\text { No lengthening } \\
\text { Increased tension }\end{array}$ & $\begin{array}{c}\text { No rotation or } \\
\text { anteroposterior } \\
\text { gliding }\end{array}$ & $\begin{array}{c}\text { CT physical } \\
\text { limit of } \\
\text { contraction } \\
\text { reached }\end{array}$ & $\begin{array}{c}\text { No } \\
\text { closure }\end{array}$ & frequencies \\
\hline
\end{tabular}

Studies have shown that when compared with untrained individuals, trained singers do not use different physiological strategies for speaking (McGlone, 1976; Allen \& Wilder, 1977; Brown 1977; McGlone, 1977; Brown et al., 1978; Watson \& Hixon, 1985; Brown Jr, et al., 1988). However, it has been noted that professional and untrained singers differ in their $F_{0}$ control strategies depending on pitch and register (Sonninen, 1968; Shipp, 1975; Shipp \& Morrissey, 1977; Fink \& Demarest, 1978; Larson, et al., 1987; Sonninen et al., 1992; Titze, 1993; Larsson \& Hertegård, 2008). As discussed above, alteration of vocal pitch is achieved by either lengthening the vocal folds or increasing their tension. To what extent each of these two mechanisms is used by singers and non-singers varies, according to different studies. However, it has been confirmed that trained singers use more forward anteroposterior gliding at the CTJ (greater pars obliqua activity) to lengthen the vocal folds to increase pitch across the vocal pitch range, compared to non-singers (Sonninen, 1968; Fink \& Demarest, 1978; Larsson \& Hertegård, 2008). Additionally, it was shown that trained singers increased vocal fold length 
with pitch up to a certain point, then increased vocal fold tension and stiffness only, more than non-singers, especially in the high pitch range (Sonninen, et al., 1992; Larsson \& Hertegård, 2008).

Equally, it has been shown that non-singers used more rotation (greater pars recta activity) to lengthen vocal folds with increased longitudinal tension across all vocal pitches (Sonninen, 1968; Fink \& Demarest, 1978; Sonninen, et al., 1992). Furthermore, it was noted that untrained singers elevated the larynx for high pitches and lowered it when singing low pitches (Dimitriev, 1962; Sundberg, 1974; Shipp \& Izdebski, 1975). This changes the vertical position of the larynx which indirectly causes increased rotation at the CTJ (Honda, 2004). In contrast, the trained singer stabilised or lowered the larynx in association with increasing frequency, so it was rarely above its resting position (Sundberg, 1974; Shipp \& Izdebski, 1975; Brown, et al., 1978; Brown Jr, et al., 1988).

As noted in the preceding discussion, pitch can be increased without necessarily changing the CT space. This implies that the starting CT space dimension is less relevant. However, the starting space may influence baseline voice characteristics which can then be modified by mechanisms other than changing the CT space. Whether the CT space is narrow or wide, vocal pitch can still be increased. Indeed in this study, the singers that had a narrow CT space still had top notes equal to their contemporaries that had a wider CT space. Sopranos with a small CT space of less than $10 \mathrm{~mm}$ could still reach top notes but had difficulty singing low notes. Difficulty singing low notes could mean they were unable to relax their CT muscles enough in the lower range, or could not activate TA enough or that the balance between CT and TA was not optimal. Singers with a smaller vocal range may not have learned optimal muscle balance. It is likely from the results of this study that most of the singers had mastered control over pitch raising mechanisms from their vocal training. This concurs with studies that have shown that vocal training increases the vocal range, especially at the upper end (Åkerlund, et al., 1992; Brown Jr, et al., 1993; Mendes, et al., 2003). 


\section{Limitations}

Some uncontrollable factors may have affected the quality of this research, particularly as the study involved the measurement of biological variables.

\subsection{General limitations}

- Sample selection and size

This study had a relatively small sample size $(n=43)$ with a limited range of voice types. Every effort was made to recruit similar numbers of individuals with each voice type and Fach. However, sopranos are more common than mezzo-sopranos, and lyric sopranos are more common than other soprano Fach types. In the final analysis there were reasonably similar numbers of different Fach types within the sopranos (4 coloraturas, 6 spintos, 10 light lyric sopranos, 5 heavy lyric sopranos, 4 soubrettes, 3 dramatic sopranos). Furthermore, compared with previous studies in this field (Vilkman, et al., 1997; Laukkanen, et al., 2002), the sample size in this study was considerably larger.

- Questionnaire results

These depend on the honesty of study participants and their nonbiased participation i.e. not changing their behaviour because they know they are participating in a study. Most of the participants and their voice types were previously known to the researcher and there was no evidence to suggest that the answers to the questionnaire were inaccurate.

\subsection{Measurement errors}

Prior to formal testing, numerous trials were conducted on all measurements, frequently retesting the same subjects to assess consistency of results. During this phase, a potential weakness was found in the measurement of neck length. Steps were taken to standardise the technique, namely the construction and use of a custom made device to ensure the head was aligned in a neutral anatomical position (see Section 5.4). 
Measuring instruments have a limited reliability i.e. its ability to give reproducible measurements when used in the same way. To minimise such measurement errors, the same equipment was used throughout the study: ultrasound machine, ultrasound transducer, scales, stadiometer, plastic tape measure and ruler.

- Ultrasound measurements

Visualising the edges of the thyroid cartilage was occasionally difficult, particularly in participants with short and/or plump necks in whom the contact between the skin and transducer probe had to be very close.

- Anthropometric measurements

Every effort was made to measure neck circumference just below the larynx and perpendicular to the axis of the neck. This was occasionally difficult in participants with a short neck.

Even though steps were taken to reduce the error in neck length measurement by utilising the Frankfurt plane for standardising head position, this may be compromised in individuals with low-set ears or any abnormality of ear shape in whom the position of the left porion could be affected. Other landmarks such as the suprasternal notch and tip of the chin were also slightly harder to define in obese participants.

- Speaking fundamental frequency results

The main limitation of SFF measurements was the method used for determining the fundamental frequency values of spoken words. Minor discrepancies may have arisen from the manual placing of the cursor when analysing frequency wave forms. Any errors may have been reduced by using a computer program to automatically generate peak wave form or average SFF values. 


\subsection{Measurement reliability}

In 14 of the 43 participants measurements were repeated after an appropriate interval and in a suitably blinded fashion to access intra-rater reproducibility. These measurements included neck circumference and length, speaking fundamental frequency, cricoid and thyroid cartilage height, and the anterior cricothyroid space (Appendix K).

The results from these repeated measurements on different occasions were not significantly different (see Results Section 6). In all 14 participants, there was less than $1 \mathrm{~cm}$ difference between the repeated neck measurements and less than $8 \mathrm{~Hz}$ difference between the repeated SFF readings. Only one laryngeal measurement differed by more than $1 \mathrm{~mm}$ and in this participant, there was difficulty accurately visualising the thyroid cartilage, which affected measurement of the CT space. However, thyroid cartilage and CT space measurements were relatively large in this case, which would tend to minimise any errors due to measurement variability.

As reported in the Results Section 6.15, strong positive correlations were attained between the first and second measurements on all re-tested parameters; neck circumference $(r=0.995 ; P<0.005)$, neck length $(r=0.985 ; P<0.005)$, speaking fundamental frequency $(r=0.989 ; P<0.005)$, cricoid cartilage $(r=0.938 ; P<0.005)$, thyroid cartilage $(r=0.983 ; P<0.005)$ and cricothyroid space $(r=0.964 ; P<0.005)$.

In summary, these measurements were highly repeatable and therefore reliable. 


\subsection{Suggestions for improvements}

This study may have been improved by streamlining the collection and analysis of data. The data acquisition process might have been easier if ultrasound images had been recorded and analysed later with fewer time constraints. Similarly, speaking fundamental frequency might also have been analysed later. However, there is no reason to believe that this procedural change would have affected the results.

\subsection{Future research}

This study showed that mezzo-sopranos had a larger CT space than sopranos. It would be interesting to explore the musical and practical implications of this observation for singers and singing practice. For example, in determining a singer's tessitura, in changing from soprano to mezzo-soprano, in warm up and practice techniques, and whether it affects durability and sustainability in prolonged periods of singing. It would also be interesting to undertake a comparative study in male singers, to see if differences are present between bass and tenor voice types. Furthermore, it appears that a narrow CT space affects how low one can sing. Further research on the impact of the CT space on potential vocal performance would be worthwhile.

The literature review (Section 2.3.1) demonstrated that numerous studies have found that senescence is associated with a reduction in total vocal range, progressive loss of top voice, and expansion of lower voice. However, several studies indicated that this trend is reduced, or even absent, in trained singers. A longitudinal study would be the best way to determine the validity of these observations.

The literature review also revealed that the physiological mechanisms behind achieving a wide range of fundamental frequencies are not completely understood. This study has shown that the CT space by itself is not a predictor of vocal range. There could be another as yet unidentified mechanism underlying $\mathrm{F}_{0}$ increase. Further investigations of 
this aspect and pitch changing mechanisms that don't involve CT space closure would be worth pursuing.

The two components of the cricothyroid (CT) muscle have been studied in detail (see Section 2.5.6). Recent literature has suggested a third (horizontal) part of this muscle (see Section 2.5.6, page 52). Further research is needed to determine the function of the component parts of this muscle and how it interacts with the pars recta and pars obliqua, and with other laryngeal muscles. It may well have a significant role in increasing $F_{0}$. Similarly, although there has been considerable research on the CT space, particularly in relation to the CTJ, CT muscle and TA muscles, there may well be other mechanisms that are involved in narrowing or opening the CT space.

The literature review also showed that both rotation and gliding movements at the CTJ vary considerably between individuals (see Section 2.5.5 and Appendix D). The function of the CTJ has a major role in determining CT space closure and hence $F_{0}$ and it may therefore be interesting to know to what extent the anatomy of the CTJ constrains the limits of the CT space.

Finally, during the course of this thesis, it became apparent that only a few reports have published normal data for SFF, neck measurements, body height and vocal range for different subcategories within each voice type. Further research is needed to establish normative data for singers according to their voice classification (including subcategories) with consideration given to age, duration of professional training, quantity and type of professional experience, hormonal factors, vocal pathology factors, cultural influences, ethnic factors, and choice of vocal tasks. 


\section{Conclusions}

This study had two aims. The first was to determine whether the anterior height of the resting cricothyroid space measured using ultrasound correlated with performance vocal range in female singers. A second aim was to investigate potential associations with and between voice categories (soprano and mezzo-soprano), age, laryngeal dimensions (anterior thyroid and cricoid cartilage heights), neck dimensions (circumference and length), anthropometric indices (weight, height, BMI), and habitual speaking fundamental frequency (SSF).

The most significant finding was that mezzo-sopranos have a wider CT space than sopranos. However, in this study sample there was no evidence to show that the CT space is a major determinant of vocal range. This is demonstrated by the fact that those singers with the narrowest and widest CT spaces had similar vocal ranges. Thus, the findings of this study do not support the hypothesis proposed by Titze (1998a) that "A wide cricothyroid space makes a wide pitch range more likely", at least in female singers.

It appears that there are individual differences in the use of $F_{0}$ control mechanisms, and that singers use vocal pitch raising mechanisms that don't simply involve closing the CT space. An extended vocal range reflects the singer's ability to use numerous strategies to enhance the vocal range. The fact that other mechanisms are involved reduces the impact of the resting CT space distance in determining vocal range. 


\section{References}

Abitbol, J., Abitbol, P., \& Abitbol, B. (1999). Sex hormones and the female voice. Journal of Voice, 13(3), 424-446.

Abitbol, J., de Brux, J., Millot, G., Masson, M.-F., Mimoun, O. L., Pau, H., \& Abitbol, B. (1989). Does a hormonal vocal cord cycle exist in women? Study of vocal premenstrual syndrome in voice performers by videostroboscopy-glottography and cytology on 38 women. Journal of Voice, 3(2), 157-162.

Abitbol, J., Timsit, C. A., \& Maimaran, J. J. (1995). Atlas of laser voice surgery. San Diego, CA: Singular Publishing Group.

Ajmani, M. L. (1990). A metrical study of the laryngeal skeleton in adult Nigerians. Journal of Anatomy, 171, 187-191.

Ajmani, M. L., Jain, S. P., \& Saxena, S. K. (1980). A metrical study of laryngeal cartilages and their ossification. Anatomischer Anzeiger, 148(1), $42-48$.

Åkerlund, L., Gramming, P., \& Sundberg, J. (1992). Phonetogram and averages of sound pressure levels and fundamental frequencies of speech: Comparison between female singers and nonsingers. Journal of Voice, 6(1), 55-63.

Alarcos, A. L., Behlau, M. S., \& Tosi, O. (1983). Computer acoustical analysis of senile voices. Folia Phoniatr, 35, 102.

Alderson, A. (1993). Positioning the velum. Journal of Research in Singing and Applied Vocal Pedagogy XVI(2), 25-32.

Allen, E., \& Wilder, C. (1977). Respiratory patterns in singers: A proposed research design. In V. Lawrence (Ed.), Transcripts of the Sixth Symposium on Care of the Professional Voice (pp. 18-20). New York: The Voice Foundation.

Altenberg, E. P., \& Ferrand, C. T. (2006). Fundamental frequency in monolingual English, bilingual English/Russian, and bilingual English/Cantonese young adult women. Journal of Voice, 20(1), 8996.

Appelman, D. R. (1967). The science of vocal pedagogy: Theory and application (Vol. 378). Bloomington, IN: Indiana University Press. 
Ardran, G. M., \& Kemp, F. H. (1966). The mechanism of the larynx. Part I: The movements of the arytenoid and cricoid cartilages. The British Journal of Radiology, 39(465), 641-654.

Arnold, G. E. (1961). Physiology and pathology of the cricothyroid muscle. The Laryngoscope, 71(7), 687-753.

Arnold, G. E. (1962). Vocal nodules and polyps: Laryngeal tissue reaction to habitual hyperkinetic dysphonia. Journal of Speech Disorders, 27(205-217).

Aronson, A. E. (1980). Clinical voice disorders: An interdisciplinary approach New York, NY: Thieme.

Atkinson, J. E. (1978). Correlation analysis of the physiological factors controlling fundamental voice frequency. Journal of the Acoustical Society of America, 63, 211-222.

Bagnall, A. D., Dorrian, J., \& Fletcher, A. (2011). Some vocal consequences of sleep deprivation and the possibility of "fatigue proofing" the voice with Voicecraft $\Theta$ voice training. Journal of Voice, 25(4), 447-461.

Baken, R. J., \& Orlikoff, R. F. (2000). Clinical measurements of speech and voice (2nd ed.). San Diego, CA: Singular Publishing Group.

Baker, S., Weinrich, B., Bevington, M., Schroth, K., \& Schroeder, E. (2008). The effect of task type on fundamental frequency in children. International Journal of Pediatric Otorhinolaryngology, 72(6), 885889.

Balasubramanian, T. (2010). Surgical management of puberphonia recent concepts. Retrieved March 18, 2013, from http://www.drtbalu.co.in/puber rec.html

Balboni, G. (1955). Biometric study of the dimensions of the human larynx. Archivio Italiano Di Anatomia E Di Embriologia 60, 161-183.

Bastian, R. W. (1996). Vocal fold microsurgery in singers. Journal of Voice, 10(4), 389-404.

Behlau, M. S., Pontes, P. A. d. L., Ganança, M. M., \& Tosi, O. (1988). Presbifonia: tratamento da deterioraçäo vocal inerente a idade. Acta Awho, 7(2), 110-115.

Behnke, E. (1900). The mechansim of the human voice (14th ed.). London, United Kingdom: Curwen, J \& Sons. 
Behrman, A. (2005). Common practices of voice therapists in the evaluation of patients Journal of Voice, 19(3), 454-469.

Ben-Noun, L. L., Sohar, E., \& Laor, A. (2001). Neck circumference as a simple screening measure for identifying overweight and obese patients. Obesity Research, 9(8), 470-477.

Benjamin, B. J. (1986). Dimensions of the older female voice. Language \& Communication, 6(1), 35-45.

Benninghoff, A., \& Drenckhahn, D. (2002). Makroskopische Anatomie, Histologie, Embryologie, Zellbiologie (Band 1, 16. Auflage).

München: Urban \& Fischer Verlag

Berry, D. A., Montequin, D. W., Chan, R. W., Titze, I. R., \& Hoffman, H. T. (2003). An investigation of cricoarytenoid joint mechanics using simulated muscle forces. Journal of Voice, 17(1), 47-62.

Boone, D. R., McFarlane, S. C., Von Berg, S. L., \& Zraick, R. I. (2010). The voice and voice therapy (8th ed.). Boston, MA: Allyn \& Bacon.

Braund, K., Steiss, J., Marshall, A., Mehta, J., \& Amling, K. (1988). Morphologic and morphometric studies of the intrinsic laryngeal muscles in clinically normal adult dogs. American Journal of Veterinary Research, 49(12), 2105.

Bricker, P. D., \& Pruzansky, S. (1976). Speaker recognition. In N. J. Lass (Ed.), Contemporary issues in experimental phonetics. New York: NY: Academic Press.

Britto, A. I., \& Doyle, P. C. (1990). A comparison of habitual and derived optimal voice fundamental frequency values in normal young adult speakers. Journal of Speech and Hearing Disorders, 55, 476-484.

Brodnitz, F. S. (1965). Vocal rehabilitation: A manual prepared for the use of graduates in medicine (3rd ed.). Rochester, MN: American Academy of Opthalmology and Otolaryngology.

Brodnitz, F. S. (1966). Rehabilitation of the human voice. Bulletin of the New York Academy of Medicine, 42(3), 231-240.

Brown Jr, W. S., Hunt, E., \& Williams, W. N. (1988). Physiological differences between the trained and untrained speaking and singing voice. Journal of Voice, 2(2), 102-110. 
Brown Jr, W. S., Morris, R. J., Hicks, D. M., \& Howell, E. (1993).

Phonational profiles of female professional singers and nonsingers. Journal of Voice, 7(3), 219-226.

Brown Jr, W. S., Morris, R. J., Hollien, H., \& Howell, E. (1991). Speaking fundamental frequency characteristics as a function of age and professional singing. Journal of Voice, 5(4), 310-315.

Brown Jr, W. S., Rothman, H. B., \& Sapienza, C. M. (2000). Perceptual and acoustic study of professionally trained versus untrained voices. Journal of Voice, 14(3), 301-309.

Brown , W. S. (1977). Supraglottal air pressure measurement as a technique for studying speech and singing. In V. Lawrence (Ed.), Transcripts of the Sixth Symposium on Care of the Professional Voice (pp. 45-47). New York: The Voice Foundation.

Brown, W. S., Rothman, H. B., \& Williams, W. N. (1978). Physiological differentiation between singers and non-singers. In V. Lawrence (Ed.), Transcripts of the Seventh Symposium on Care of the Professional Voice (pp. 11-18). New York: The Voice Foundation.

Callaghan, J. (2000). Singing and voice science. San Diego, CA: Singular Publishing Group

Chagnon, F. P. (1998, September). Classifying singing voices. La Scena Musicale, 4(1). Retrieved from http://www.scena.org/lsm/sm41/sm4-1VoiceDoc en.htm

Chapman, J. L. (2006). Singing and teaching singing: A holistic approach to classical voice. San Diego, CA: Plural Publishing.

Chen, Y., Kimelman, M. D. Z., \& K., M. (2009). Investigation of habitual pitch during free play activities for preschool-aged children. International Journal of Pediatric Otorhinolaryngology, 73, 73-80.

Cheng, Y., Leow, W. K., \& Lim, T. C. (2012). Automatic identification of Frankfurt Plane and mid-sagittal plane of skull. Paper presented at the Applications of Computer Vision (WACV), 2012 IEEE Workshop, Breckenridge, CO. Retrieved from http://www.comp.nus.edu.sg/ cyuan/publications/WACV2012skull.p $\underline{\mathrm{df}}$

Chevrie-Muller, C., Salomon, D., \& Ferrey, G. (1971). Contribution a l'établissement de quelques constantes physiologiques de la voix parlée de la femme adolescente, adulte et age. Journal Français d'Oto-Rhino-Laryngologie, XVI, 433-455. 
Chiba, T., \& Kajiyama, M. (1941). The vowel: Its nature and structure. Tokyo, Japan: Kaiseikan.

Chievitz, J. H. (1882). Unter suchungen uber die verknocherung. Archives für Anatomie und Physiologie.

Cleveland, T. F. (1977). Acoustic properties of voice timbre types and their influence on voice classification. Journal of Acoustic Society of America, 61, 1622-1629.

Cleveland, T. F. (1991). Does the premier singer's larynx show visible differences from the normal singer's larynx? Journal of Voice, 1991(7), 33.

Coleman, R. F. (1993). Sources of variation in phonetograms. Journal of Voice, 7(1), 1-14.

Coleman, R. F., \& Markham, I. W. (1991). Normal variations in habitual pitch. Journal of Voice, 5(2), 171-177.

Collins, S. A. (2000). Men's voices and women's choices. Animal Behaviour, 60(6), 773-780.

Colton, R. H. (1988). Physiological mechanisms of vocal frequency control: The role of tension. Journal of Voice, 2(3), 208-220.

Colton, R. H., Casper, J. K., \& Leonard, R. (2011). Understanding voice problems: A physiological perspective for diagnosis (4th Revised edition ed.). Philadelphia, PA: Lippincott Williams and Wilkins.

Darwin, C. (1871). The Descent of Man and selection in relation to sex. London, UK: Murray.

De Pinto, O., \& Hollien, H. (1982). Speaking fundamental frequency characteristics of Australian women: then and now. J Phon, 10, 367-375.

Debruyne, F., Decoster, W., Van Gijsel, A., \& Vercammen, J. (2002). Speaking fundamental frequency in monozygotic and dizygotic twins. Journal of Voice, 16(4), 466-471.

Decoster, W., \& Debruyne, F. (1997). Changes in spectral measures and voice-onset time with age: a cross-sectional and a longitudinal study. Folia Phoniatrica et Logop 49, 269-280. 
Deguchi, S., Kawahara, Y., \& Takahashi, S. (2011). Cooperative regulation of vocal fold morphology and stress by the cricothyroid and thyroarytenoid muscles. Journal of Voice, 25(6), e255-e263.

Dimitriev, L. (1962). Golosoorasovanie i pevstsov: Gosidarstvennoe musikalnoe isdatelstvo. Moscow, Russia.

Donald, P. J. (1982). Voice change surgery in the transsexual. Head and Neck Surgery, 4, 433-437.

Doscher, B. (1994). The functional unity of the singing voice (2nd ed.). Metuchen, NJ: Scarecrow Press.

Drake, R. L., Vogl, W., \& Mitchell, A. W. M. (2005). Gray's anatomy for students flash cards. New York, NY: Churchill Livingstone.

Drew, R., \& Sapir, S. (1995). Average speaking fundamental frequency in soprano singers with and without symptoms of vocal attrition. Journal of Voice, 9(2), 134-141.

Duffy, R. (1970). Fundamental frequency characteristics of adolescent females. Language and Speech, 13, 14-24.

Eckel, H. E., \& Sittel, C. (1995). Morphometry of the larynx in horizontal sections. American Journal of Otolaryngology, 16(1), 40-48.

Eckel, H. E., Sittel, C., Zorowka, P., \& Jerke, A. (1994). Dimensions of the laryngeal framework in adults. Surgical and Radiologic Anatomy, 16(1), 31-36.

Evans, S., Neave, N., \& Wakelin, D. (2006). Relationships between vocal characteristics and body size and shape in human males: An evolutionary explanation for a deep male voice. Biological Psychology, 72(2), 160-163.

Fairbanks, G. (1959). Voice and articulation drillbook. New York, NY: Harper \& Row.

Fairbanks, G., Herbert, E., \& Hammond, J. (1949). An acoustical study of vocal pitch in seven- and eight-year old girls. Child Development, 20, 71-78.

Fairbanks, G., Wiley, J. H., \& Lassman, F. (1949). An acoustical study of vocal pitch in seven- and eight-year-old boys. Child Development, 20, 63-69. 
Filho, J. A. X., Bohadana, S. C., Perazzio, A. F., Tsuji, D. H., \& Sennes, L. U. (2005). Anatomy of the cricothyroid articulation: Differences between men and women Annals of Otology, Rhinology and Laryngology 114(3), 250-252

Fink, B. R. (1962). Tensor Mechanism of vocal folds. Annals of Otology, Rhinology and Laryngology 71, 591-601.

Fink, B. R., \& Demarest, R. J. (1978). Laryngeal Biomechanics. Cambridge, MA: Harvard Univeristy Press.

Fitch, J. L. (1990). Consistency of fundamental frequency and perturbation in repeated phonations of sustained vowels, reading, and connected speech. Journal of Speech and Hearing Disorders, 55, 360-363.

Fitch, W. T., \& Giedd, J. (1999). Morphology and development of the human vocal tract: a study using magnetic resonance imaging. Journal of Acoustical Society of America, 106(3 Pt 1), 1511-1522.

Freedman, L. M. (1956). The role of the cricothyroid muscle in tension of the vocal cord. Laryngoscope, 66, 574-581.

Friedrich, G., Remacle, M., Birchall, M., Marie, J. P., \& Arens, C. (2007). Defining phonosurgery: a proposal for classification and nomenclature by the Phonosurgery Committee of the European Laryngological Society (ELS). European Archives of Otorhinolaryngology, 264(10), 1191-2000.

Gay, T., Strome, M., Hirose, H., \& Sawashima , M. (1972). Electromyography of the intrinsic laryngeal muscles. Annals of Otolaryngology, Rhinology and Laryngology, 81, 401-409.

Genack, S. H., Woo, P., Colton, R. H., \& Goyette, D. (1993). Partial thyroarytenoid myectomy: an animal study investigating a proposed new reatment for adductor spasmodic dysphonia. Otolaryngology Head and Neck Surgery, 108, 256-264.

Gilbert, H. R., \& Weismer, G. G. (1974). The effects of smoking on the speaking fundamental frequency of adult women. Journal of Psycholinguistic Research, 3, 225-231.

González, J. (2003). Estimation of speakers' weight and height from speech: A re-analysis of data from multiple studies by Lass and colleagues. Perceptual and Motor Skills, 96, 297-304. 
Goumas, P., Kokkinis, K., Petrocheilos, J., Naxakis, S., \& Mochloulis, G. (1997). Cricothyroidotomy and the anatomy of the cricothyroid space. An autopsy study. The Journal of Laryngology and Otology., 111(4), 3

Graddol, D., \& Swann, J. (1983). Speaking Fundamental Frequency: Some Physical and Social Correlates. Language and Speech, 26(4), 351-366.

Gray, H. (1959). Anatomy of the human body (27th ed.). Philadelphia: Lea and Febiger.

Greene, M. (1972). The voice and its disorders. London, New York: Pitman pp32-105.

Gross, M. (1999). Pitch-raising surgery in male-to-female transsexuals. Journal of Voice, 13(2), 246-250.

Gross, M., \& Fehland, P. (1995). Results after surgical raising the average speaking voice for transsexuals position by shortening the vibrating vocal folds share Berlin 1996.

Gugatschka, M., Kiesler, K., Chibidziura-Priesching, J., Schökler, B., \& Friedrich, G. (2009). Pitch synchronous changes of the anterior cricothyroid gap by using ultrasonography. Journal of Voice, 23(5), 610-613.

Gunter, C., \& Manning, W. (1982). Listener estimations of speaker height and weight in unfiltered and filtered conditions. Journal of Phonetics, 10, 251-257.

Gutzmann, H. (1949). Über das Erkennen der Stimmgattung: On the identification of voice classification. Folia Phoniatrica (Basel), 2, 1-5.

Haglund, S. (1973). The normal electromyogram in human cricothyroid muscle. Acta Oto-Laryngologica, 75(2-6), 448-453.

Hamdan, A.-L., Al-Barazi, R., Tabri, D., Saade, R., Kutkut, I., Sinno, S., \& Nassar, J. (2012). Relationship between acoustic parameters and body mass analysis in young males. Journal of Voice, 26(2), 144147.

Hammer, G. P., Windisch, G., Prodinger, P. M., Anderhuber, F., \& Friedrich, G. (2010). The cricothyroid joint: Functional aspects with regard to different types of its structure. Journal of Voice, 24(2), 140-145. 
Hanley, T. D. (1951). An analysis of vocal frequency and duration characteristics of selected sample of speech from General American, Eastern American and Southern American dialect regions. Speech Monographs, 18, 78-93.

Harjeet, \& Jit, I. (1992). Dimensions of the thyroid cartilage in neonates, children and adults in north west Indian subjects. Journal of the Anatomical Society of India, 41(2), 81-92.

Harnsberger, J. D., Shrivastav, R., Brown Jr, W. S., Rothman, H., \& Hollien, H. (2008). Speaking rate and fundamental frequency as speech cues to perceived age. Journal of Voice, 22, 58-69.

Harries, M., Hawkins, S., Hacking, J., \& Hughes, I. (1998). Changes in the male voice at puberty vocal fold length and its relationship to the fundamental frequency of the voice. Journal of Laryngology and Otolaryngology, 112(5), 451-454.

Harris, T., Harris, S., Rubin, J. S., \& Howard, D. M. (1998). The voice clinic handbook: Whurr Publishers, London

Hass, J. (2003). An acoustics primer. Retrieved 9 January 2012, from http://www.indiana.edu/ emusic/acoustics/frequency.htm

Hast, M. H. (1966). Mechanical properties of the cricothyroid muscle. The Laryngoscope, 76(3), 537-548.

Henderson, R. (1954). Kathleen Ferrier 1912-1953, A memoir. London.

Henrich, N. (2006). Mirroring the voice from Garcia to the present day: Some insights into singing voice registers. Logopedics Phoniatrics Vocology, 31(1), 3-14.

Henrich, N., d'Alessandro, C., Doval, B., \& Castellengo, M. (2005). Glottal open quotient in singing: Measurements and correlation with laryngeal mechanisms, vocal intensity, and fundamental frequency. The Journal of the Acoustical Society of America, 117, 1417.

Hertegård, S., Hakansson, A., \& Thorstensen, O. (1993). Vocal fold length measurements with computed tomography. Logopedics Phoniatrics Vocology, 18(2), 57-63.

Higgins, M. B., \& Saxman, J. H. (1989). Variations in vocal frequency perturbation across the menstrual cycle. Journal of Voice, 3(3), 233243. 
Higgins, M. B., \& Saxman, J. H. (1991). Comparsion of selected phonatory behaviours of healthy aged and young adults. Journal of Speech and Hearing Research, 34, 1000-1010.

Hirano, M. (1974). Morphological structure of the vocal cord as a vibrator and its variations. Folia Phoniatrica Logopaedica, 26, 89-94.

Hirano, M. (1975). Phonosurgery: Basic and clinical investigations. . Otologia (Fukuoka) 21, 239-442.

Hirano, M. (1977). Structure and vibratory behavior of the vocal folds. In M. Sawashima \& S. C. Franklin (Eds.), Dynamic aspects of speech production (pp. 13-30). Tokyo: University of Tokyo Press.

Hirano, M. (1981). The function of the intrinsic laryngeal muscles in singing. In K. Stevens \& M. Hirano (Eds.), Vocal fold physiology (pp. 13-20). Tokyo, Japan: University of Tokyo Press.

Hirano, M. (1989). Objective evaluation of the human voice: clinical aspects. Folia Phoniatrica, 41(2-3), 89-144.

Hirano, M., Kurita, S., \& Nakasbirna, T. (1981). The structure of the vocal folds. In K. N. Stevens \& M. Hirano (Eds.), Vocal fold physiology (XIV $421 \mathrm{~S}$ ed.). Tokyo, Japan: University of Tokyo Press. pp. 33-41.

Hirano, M., Matsuo, K., Kakita, Y., Kawasaki, H., \& Kurita, S. (1983, May 4-7). Vibratory behavior versus the structure of the vocal fold. Paper presented at the International Conference on the Physiology and Biophysics of Voice, University of lowa, lowa City, lowa.

Hirano, M., Ohala, J., \& Vennard , W. (1969). The function of laryngeal muscles in regulating fundamental frequency and intensity of phonation. Journal of Speech and Hearing Research, 12, 616-628.

Hirano, M., \& Sato, K. (1993). Histological color atlas of the human larynx. San Diego, CA: Singular Publishing.

Hirano, M., Vennard, W., \& Ohala, J. (2009). Regulation of register, pitch and intensity of voice. Folia Phoniatrica et Logopaedica, 22(1), 1-20.

Hiroto, I., Hirano, M., Toyozumi, Y., \& Shin, T. (1967). Electromyographic investigation of the intrinsic laryngeal muscles related to speech sounds. Annals of Otology, Rhinology and Laryngology 76, 861-872.

Hollien, H. (1960). Some laryngeal correlates of vocal pitch. Journal of Speech and Hearing Research, 3, 52-58. 
Hollien, H. (1993). That golden voice: Talent or training? Journal of Voice, 7(3), 195-205.

Hollien, H., Dew, D., \& Phillips, P. (1971). Phonational frequency ranges of adults. Journal of Speech and Hearing Research, 14, 755-760.

Hollien, H., Green, R., \& Massey, K. (1994). Longitudinal research on adolescent voice change in males. Journal of the Acoustical Society of America, 96, 2646-2653.

Hollien, H., \& Malcik, E. (1962). Adolescent voice changes in southern negro males. Speech Monographs, 29, 53-58.

Hollien, H., \& Michel, J. F. (1968). Vocal Fry as a Phonational Register. Journal of Speech and Hearing Research, 11(3), 600-604.

Hollien, H., \& Shipp, T. (1972). Speaking fundamental frequency and chronological age in males. Journal of Speech and Hearing Research, 15, 155-159.

Honda, K. (1983). Variability analysis of laryngeal muscle activities. In I. R. Titze \& R. Scherer (Eds.), Vocal Fold Physiology: Biomechanics, Acoustics, and Phonatory Control (pp. 286-297). Denver, CO: The Denver Centre for the Performing Arts.

Honda, K. (1988). Various larngeal mechansims in controlling the voice fundamental frequency. Journal of the Acoustical Society of America, 84(Suppl. 1, S82).

Honda, K. (2004, March 23-26). Physiological factors causing tonal characteristics of speech: From global to local prosody. Paper presented at the Speech Prosody 2004, Nara, Japan.

Hong, K. H., Kim, H. K., \& Kim, Y. M. (2001). The role of the pars recta and pars oblique of cricothyroid muscle in speech production. Journal of Voice, 15, 512-518.

Hong, K. H., Ye, M., Kim, Y. M., Kevorkian, K. F., Kreiman, J., \& Berke, G. S. (1998). Functional differences between the two bellies of the cricothyroid muscle. American Academy of Otolaryngology - Head and Neck Surgery Foundation, 118(5), 714-722.

Honjo, I., \& Isshiki , N. (1980). Laryngoscopic and voice characteristics of aged persons. Archives of Otolaryngology, 106, 149-150. 
Horii, Y. (1975). Some statistical characteristics of voice fundamental frequency. Journal of Speech and Hearing Research, 18(1), 192201.

Horii, Y. (1982). Some voice fundamental frequency characteristics of young black adults: spontaneous speaking and oral reading. Journal of Speech and Hearing Research, 25, 608-210.

How Music Works. (2012). Sound and music. Retrieved 16 September 2012, from http://www.howmusicworks.org/hmw100.html

Hsiao, J., \& Pacheco-Fowler, V. (2008). Cricothyroidotomy. New England Journal of Medicine, 358(22), e25.

Hudson, A. I., \& Holbrook, A. (1982). Fundamental frequency characteristics of young black adults: spontaneous speaking and oral reading. Journal of Speech and Hearing Research, 25, 25-28.

Hunter, E. J. (2009). A comparison of a child"s fundamental frequencies in structured elicited vocalizations versus unstructured natural vocalizations: A case study. International Journal of Pediatric Otorhinolaryngology, 73, 561-571.

Isshiki, N. (1981). Phonosurgery to change vocal pitch. Hals Nasen Ohrenheilkunde, Praxis (Leipzig), 6, 179-180.

Isshiki, N. (1989). Phonosurgery: Theory and practice. Surgery to elevate vocal pitch. Berlin, Germany: Springer.

Isshiki, N., Haji, T., Yamamoto, Y., \& Mahieu, H. F. (2001). Thyroplasty for adductor spasmodic dysphonia: further experiences. The Laryngoscope, 111(4), 615-621.

Isshiki, N., Morita, H., Okamura, H., \& Hiramoto, M. (1974). Thyroplasty as a new phonosurgical technique. Acta Otolaryngologica, 78(5-6), 451-457.

Isshiki, N., Taira, T., \& Tanabe, M. (1983). Surgical alteration of the vocal pitch. The Journal Of Otolaryngology, 12(5), 335-340.

Jain, M., \& Dhall, U. (2008). Morphometry of the thyroid and cricoid. Journal of Anatomical Society of India, 57(2), 119-123.

Jewett, S. O. (1869). Parlor singing. The Atlantic Monthly, 24(October), 10.

Jiang, J., Lin, E., \& Hanson, D. G. (2000). Vocal fold physiology. Otolaryngologic Clinics of North America, 33(4), 699-718. 
Judson, L. S., \& Weaver, A. T. (1942). Vocal Science. New York, NY: Crofts, F.S.

Kahane, J. C. (1978). A morphological study of the human prepubertal and pubertal larynx. [Research Support, U.S. Gov't, P.H.S.]. The American Journal of Anatomy, 151(1), 11-19.

Kahane, J. C. (1994). A contemporary look at the cricothyroid joint. Paper presented at the Special Session at the 23rd Annual Symposium: Care of the Professional Voice, Philadelphia, Pennsylvania.

Kitajima, K., Tanabe, M., \& Isshiki, N. (1979). Cricothyroid distance and vocal pitch. Experimental surgical study to elevate the vocal pitch. Annals of Otology, Rhinology and Laryngology, 88(1 Pt 1), 52-55.

Kitzing, P. (1979). Glottografisk frekvensindikering. Lund University, Sweden.

Klimek, M. M., Obert, K., \& Steinhauer, K. (2005). Estill voice training system: Level One: Think Voice International.

Kocak, I., Dogan, M., Tadihan, E., Alkan Cakir, Z., Bengisu, S., \& Akpinar, M. (2008). Window anterior commissure relaxation laryngoplasty in the management of high-pitched voice disorders. Archives of Otolaryngology Head and Neck Surgery, 134(12), 1263-1269.

Kochis-Jennings, K. A., Finnegan, E. M., Hoffman, H. T., \& Jaiswal, S. (2012). Laryngeal muscle activity and vocal fold adduction during chest, chestmix, headmix, and head registers in females. Journal of Voice, 26(2), 182-193.

Kokawa, N. (1977). A new surgical procedure for dysphonia due to androgenic or anabolic hormones. Journal of Japanese Bronchoesoph society, 28, 323-332.

Koufman, J. A., \& Blalock, P. D. (1988). Vocal fatigue and dysphonia in the professional voice user: Bogart-Bacall syndrome. Laryngoscope, May;98((5)), 493-498.

Koufman, J. A., \& Isacson, G. (1991). Laryngoplastic phonosurgery. Otolaryngologic Clinics of North America(24), 1151-1177.

Krauss, R. M., Freyberg, R., \& Morsella, E. (2002). Inferring speakers' physical attributes from their voices. Journal of Experimental Social Psychology, 38, 618-625. 
Kreiman , J. (1997). Listening to voices: theory and practice in voice perception research. In K. Johnson \& J. W. Mullennix (Eds.), Talker variability in speech processing. San Diego, CA: Academic Press.

Kunachak, S., Prakunhungsit, S., \& Sujjalak, K. (2000). Thyroid cartilage and vocal fold reduction: a new phonosurgical method for male-tofemale transsexuals. The Annals of Otology, Rhinology, and Laryngology, 109(11), 1082-1086.

Kunzel, H. J. (1989). How well does average fundamental frequency correlate with speaker height and weight? Phonetica, 46(1-3), 117125.

Lanz, v. T., \& Wachsmuth, W. (1955). Praktische Anatomie (Vol. 1, pp. 272-292). Berlin, Germany: Springer.

Larson, C. R., Kempster, G. B., \& Kistler, M. K. (1987). Changes in voice fundamental frequency following discharge of single motor units in cricothyroid and thyroarytenoid muscles. Journal of Speech, Language and Hearing Research, 30(4), 552-558.

Larsson, H., \& Hertegård, S. (2008). Vocal Fold Dimensions in Professional Opera Singers as Measured by Means of Laser Triangulation. Journal of Voice, 22(6), 734-739.

Lass, N. J., Barry, P. J., Reed, R. A., Walsh, J. M., \& Amusto, T. A. (1979). The effect of temporal speech alterations on speaker height and weight identification. Language \& Speech, 22, 163-171.

Lass, N. J., Beverly, A. S., Nicosia, D. K., \& Simpson, L. A. (1977). An investigation of speaker height and weight identification: II. Direct estimations. Journal of the Acoustical Society of America, 61(S1), S70.

Lass, N. J., \& Brown, W. S. (1978). Correlational study of speakers' heights, weights, body surface areas, and speaking fundamental frequencies. Journal of the Acoustical Society of America, 63(4), 1218-1220.

Lass, N. J., \& Colt, E. G. (1980). A comparative study of the effect of visual and auditory cues on speaker height and weight identification. Journal of Phonetics, 8, 277-285.

Lass, N. J., \& Davis, M. (1976). An investigation of speaker height and weight identifcation. Journal of the Acoustical Society of America, 60, 700-703. 
Lass, N. J., DiCola, G. A., Beverly, A. S., Barbera, C., Henry, G. H., \& Badali, M. K. (1979). The effect of phonetic complexity on speaker height and weight identification. Language \& Speech, 22, 297-309.

Laukkanen, A.-M., Takalo, R., Arvonen, M., \& Vilkman, E. A. (2002). Pitchsynchronous changes in the anterior cricothyroid space during singing. Journal of Voice, 16(2), 182-194.

Laver, J., \& Trudgill, P. (1979). Phonetic and linguistic markers in speech. In R. Scherer \& Giles (Eds.), Social markers in speech (pp. 1-32). Cambridge, UK: Cambridge University Press.

Lawrence, A. (2004). Voice feminisation surgery: An overview. Retrieved 12 November 2011, from http://www.annelawrence.com/voicesurgery.html

LeJeune, F. E., Guice, C. E., \& Samuels, P. M. (1983). Early experiences with vocal ligament tightening. Annals of Otology, Rhinology and Laryngology 92, 475-477.

Lindestad, P.-Å., Fritzell, B., \& Persson, A. (1991). Quantitative analysis of laryngeal EMG in normal subjects. Acta Oto-Laryngologica, 111(3), 1146-1152.

Lindh, J. (2006). Preliminary FO statistics and forensic phonetics. Paper presented at the IAFPA, Department of Linguistics, Göteborg University, Sweden.

Linville, S. E. (1987). Maximum phonational frequency range capabilities of women's voices with advancing age. Folia Phoniatrica (Basel), 39(6), 297.

Linville, S. E., \& Fisher, H. B. (1985). Acoustic characteristics of perceived versus actual vocal age in controlled phonation by adult females. Journal of the Acoustical Society of America, 78, 40-48.

Little, M. (2012). The voice detective. Retrieved 13 October 2013, from http://blog.ted.com/2012/12/21/the-voice-detective-fellows-fridaywith-max-little/

Longia, G. S. (1990). Anthropometrical features of laryngeal cartilges. Journal of Forensic Medicine (Istanbul), 6, 141-148.

Longia, G. S., \& Saxena, S. K. (1980). Anthropometrical features of thyroid and cricoid cartilage. Journal of Anatomical Science, 2, 33-40. 
Mahieu, H. F., \& Schutte, H. K. (1989). New surgical techniques for voice improvement. European Archives of Oto-Rhino-Laryngology, 246(5), 397-402.

Malinowski, A. (1967). Shape, dimensions and process of calcification of the cartilaginous framework of the larynx in relation to age and sex in the Polish population. Folia Morphologica, 26(2), 121.

Manén, L. (1974). The art of singing. London, England: Faber Music.

Matai, V., Cheesman, A. D., \& Clarke, P. M. (2003). Cricothyroid approximation and thyroid chondroplasty: A patient survey. Otolaryngology - Head and Neck Surgery, 128(6), 841-847.

Maue, W. M., \& Dickson, D. R. (1971). Cartilages and ligaments of the adult human larynx. Archives of Otolaryngology, 94, 432-439.

Mayet, A., \& Mundnich, K. (1958). Contribution to the anatomy and functions of the cricothyreoideus muscle and articulation. Acta Anatomica (Basel), 33(4), 273-288.

McCoy, S. (2004). Your voice : An inside view. Princeton, NJ: Inside View Press.

McGinnis, P. Y. (2010). The opera singers career guide: Understanding the European fach system. Lanham, MD: Scarecrow Press.

McGlone, R. E. (1976). Lingual pressure variation during singing by trained and untrained individuals. Paper presented at the Fifth Symposium on Care of the Professional Voice, The Juilliard School, New York.

McGlone, R. E. (1977). Supraglottal air pressure variation from trained singers while speaking and singing. In V. Lawrence (Ed.), Transcripts of the Sixth Symposium on Care of the Professional Voice (pp. 48-49). New York, NY: The Voice Foundation.

McGlone, R. E., \& Hollien, H. (1963). Vocal pitch characteristics of aged women. Journal of Speech, Language and Hearing Research, 1963(6), 164-170.

McHenry, M. A., Kuna, S. T., Minton, J. T., Vanoye, C. R., \& Calhoun, K. (1997). Differential activity of the pars recta and pars oblique in fundamental frequency control. Journal of Voice, 11, 48-58. 
Mendes, A. P., Rothman, H. B., Sapienza, C., \& Brown Jr, W. S. (2003). Effects of vocal training on the acoustic parameters of the singing voice. Journal of Voice, 17(4), 529-543.

Messing, B. (2012). Anatomy and physiology of the voice. Retrieved March 19, 2012, from http://www.gbmc.org/home voicecenter.cfm?id=1552

Michael, D. D. (2012). About the voice: How does your voice work? Retrieved March 19, 2012, from http://www.lionsvoiceclinic.umn.edu/page2.htm

Miller, D. G. (2008). Resonance in singing. Princeton, NJ: Inside View Press.

Miller, R. (1986a). The role of the jaw in singing. Journal of Voice, 43, 2932.

Miller, R. (1986b). The structure of singing. New York, NY Schirmer Books. pp.161-170, 205-292.

Miller, R. (2000). Training soprano voices. New York, NY: Oxford University Press. pp 1-28.

Minnigerode, B. (1955). Messungen über die lage einiger auf den schildknorpel projinzieter teile des kehlkopfinneren. Hals Nasen Ohrenheilkunde (Berlin), 5, 51-56.

Minnigerode, B. (1967). Study on the importance of extralaryngeal muslces for the act of phonation with specail reference to the cricopharyngeal muscle. Archiv Fur Klinische Und Experimentelle Ohren-, Nasen- Und Kehlkopfheilkunde 188, 604-623.

Moeller, J., \& Fisher, J. F. (1904). Observation on the action of the crocothyroideus and thyroarytenoideusinternus. Archives Laryngology and Rhinology, 15(72).

Morris, R. J., \& Brown Jr, W. S. (1987). Age-related voice measures among adult women. Journal of Voice, 1(1), 38-43.

Morris, R. J., \& Brown Jr, W. S. (1996). Comparison of various automatic means for measuring mean fundamental frequency. Journal of Voice, 10(2), 159-165.

Morris, R. J., Brown Jr, W. S., Hicks, D. M., \& Howell, E. (1995). Phonational profiles of male trained singers and nonsingers. Journal of Voice, 9(2), 142-148. 
Moses, P. (1949). Vocal analysis. Archives of Otolaryngology, 48, 171.

Mu, L., \& Sanders, I. (2007). The human cricothyroid muscle: Three muscle bellies and their innervation patterns. Journal of Voice, 23(1), 21-28.

Mueller, P., Sweeney, R., \& Baribeau, L. (1984). Acoustic and morphologic study of the senescent voice. Ear, Nose, \& Throat Journal, 63(6), 292-295.

Mürbe, D., Roers, F., \& Sundberg, J. (2011). Voice classification in professional singers: The influence of vocal fold length, vocal tract length and body measurements. Hals Nasen Ohrenheilkunde, 59(6), 7.

Murry, T. (1978). Speaking fundamental frequency characteristics associated with voice pathologies. Journal of Speech and Hearing Disorders, 43(3), 374-379.

Murry, T., Brown, W. S., \& Morris, R. J. (1995). Patterns of fundamental frequency for three types of voice samples. Journal of Voice, 9 , 282-289.

Murry, T., \& Doherty, E. T. (1980). Selected acoustic characteristics of pathological and normal speakers. Journal of Speech and Hearing Research, 23(2), 361-369.

Mysak, E. D. (1959). Pitch and duration characteristics of older males. Journal of Speech and Hearing Research, 2, 46-54.

Nadoleczny, M. (1923). Studies on the art song (Untersuchwingen uber den kunstgesang). Berlin, Germany: Springer.

National Center for Voice and Speech. (2013a). How humans control pitch. Retrieved June 30, 2013, from http://www.ncvs.org/ncvs/tutorials/voiceprod/tutorial/cover.html

National Center for Voice and Speech. (2013b). Voice changes throughout life. Retrieved June 30, 2013, from http://www.ncvs.org/ncvs/tutorials/voiceprod/tutorial/changes.html

Negus, V. (1949). The comparative anatomy of the larynx. London, UK: Heinemann.

Neumann, K., \& Welzel, C. (2004). The importance of the voice in male-tofemale transsexualism. Journal of Voice, 18(1), 153-167. 
Neumann, K., Welzel, C., \& Berghaus, A. (2002). Cricothyroidopexy in male-to-female-transsexuals: Modification of Thyroplasty Type IV. The International Journal of Transgenderism, 6(3).

Nishizawa, N., Sawashima, M., \& Yonemoto, K. (1988). Vocal fold length in vocal pitch change. In O. Fujimura (Ed.), Vocal physiology: Voice production mechansims and functions (Vol. 2, pp. 75-83). New York, NY: Raven Press.

O'Connor, K. (2012). Sing Wise. Retrieved June 6, 2013, from http://www.singwise.com/index.php

Orloff, L. A., Mann, A. P., Damrose, J. F., \& Goldman, S. N. (2006). Laserassisted voice adjustment (LAVA) in transsexuals. Laryngoscope, $116(4), 655-660$.

Pedersen, M., \& McGlashan, J. (2010). Surgical versus non-surgical interventions for vocal cord nodules (Review). The Cochrane Library(3), 15.

Pegoraro- Krook, M. I. (1988). Speaking fundamental frequency characterisitcs of normal Swedish sunjects obtained by glottal frequency analysis. Folia Phoniatrica, 40, 82-90.

Peppard, R. C., Bless, D. M., \& Milenkovic, P. (1988). Comparison of young adult singers and nonsingers with vocal nodules. Journal of Voice, 2(3), 250-260.

Perlman, A. L., Titze, I. R., \& Cooper, D. S. (1984). Elasticity of canine vocal fold tissue. Journal of Speech, Language and Hearing Research, 27(2), 212.

Pešák, J. (2009). Complex Mechanism of Laryngeal Phonation. Folia Phoniatrica et Logopaedica, 42(4), 201-207.

Pfau, W. (1973). Klassifizierung der menschlichen Stimme. Leipzig, Germany: J. A. Barth.

Proctor, D. F. (1980). Breathing, Speech and Song. New York, NY: Springer.

Raj, A., Gupta, B., Chowdhury, A., \& Chadha, S. (2010). A study of voice changes in various phases of menstrual cycle and in postmenopausal women. Journal of Voice, 3, 363-368. 
Ramig, L. A., \& Ringel, R. L. (1983). Effects of physiological aging on selected acoustic characteristics of voice. Journal of Speech and Hearing Research, 26(1), 22-30.

Ranke, O. F., \& Lullies, H. (1953). Gehor-Stimme-Sprache. Berlin, Germany: Springer.

Reeve, M. (2005). The Bernoulli effect and vocal fold vibration. Retrieved 19th April 2012, from http://www.voicesource.co.uk/article/151

Reidenbach, M. M. (1996). The cricothyroid space: Topography and clinical implications. Acta Anatomica, 157, 9.

Remacle, M., Matar, N., Verduyckt, I., \& Lawson, G. (2010). Relaxation thyroplasty for mutational falsetto treatment. Annals of Otology, Rhinology and Laryngology 119(2), 105-109.

Ricci-Maccarini, A., Pieri, F., \& Magnani, M. (2011). Phonosurgical options in transexual patients. Cesena, Itlay: ENT Department, Phoniatrics and Phonosurgery Module, Bufalini Hospital.

Roers, F. (2005). Morphological and physical differences between vocal students of different kinds of voices. MD Thesis, Dresden University of Technology Dresden

Roers, F., Mürbe, D., \& Sundberg, J. (2007). Predicted singers' vocal fold lengths and voice classification: A study of X-Ray morphological measures. Journal of Voice, 23(4), 408-413.

Roers, F., Mürbe, D., \& Sundberg, J. (2009). Voice classification and vocal tract of singers: A study of x-ray images and morphology. Journal of the Acoustical Society of America, 125(1), 503.

Rosen, C. A., \& Simpson, C. B. (2008). Operative techniques in laryngology. Berlin, Germany: Springer.

Roubeau, B., Castellengo, M., Bodin, P., \& Ragot, M. (2004). Laryngeal registers as shown in the voice range profile. Folia Phoniatrica Logopaedica, 56(5), 321-333.

Roubeau, B., Chevrie-Muller, C., \& Saint Guily, J. L. (1997).

Electromyographic activity of strap and cricothyroid muscles in pitch change. Acta Oto-Laryngologica, 117(3), 459-464.

Rubin, W. (1988). Allergic, dietary, chemical, stress, and hormonal influences in voice abnormalities. Journal of Voice, 1(4), 378-385. 
Russell, A., Penny, L., \& Pemberton, C. (1995). Speaking fundamental frequency changes over time in women: a longitudinal study. Journal of Speech, Language and Hearing Research, 38(1), 101.

Saito, S. (1977). Phonosurgery. Otologica, 23(Supplement I), 171-384.

Sappey, M. P. C. (1874). Treatise on descriptive anatomy (2nd ed. Vol. 4). Paris, France: Delahaye 382-420.

Sataloff, R. T. (1995). Genetics of the voice. Journal of Voice, 9(1), 16-19.

Sataloff, R. T. (1997). The Proffessional Voice: The science and art of clinical care (2nd ed.). San Diego, CA: Singular Publishing Group.

Sataloff, R. T., Caputo Rosen, D., Hawkshaw, M., \& Spiegel, J. R. (1997). The aging adult voice. Journal of Voice, 11(2), 156-160.

Sataloff, R. T., Rao, V. M., Hawkshaw, M., Lyons, K., \& Spiegel, J. R. (1998). Cricothyroid joint injury. Journal of Voice, 12(1), 112-116.

Sataloff, R. T., Spiegel, J. R., Carroll, L. M., \& Heuer, R. J. (1992). Male soprano voice: A rare complication of thyroidectomy. Laryngoscope, 102(1), 90-93.

Schade, G., Hess, M., \& Rassow, B. (2002). Possibility for endolaryngeal morphometric measurements with a new laser light method. Hals Nasen Ohrenheilkunde, 50(8), 753-755.

Scherer, R. C., \& Guo, C. G. (1990, November 18-22). Laryngeal modeling: Translaryngeal pressure for a model with many glottal shapes. Paper presented at the First International Conference on Spoken Language Processing, Kobe, Japan.

Schiwitz, A. C. (2011). The effect of task type on speaking fundamental frequency in women. Master of Science, (Thesis) Auburn University, Auburn, Alabama.

Schultz-Coulon, H. J. (1975). Bestimmung und Beurteilung der individuellen mittleren Sprechstimmlage. Folia Phoniatrica, 27(5), 375-386.

Schünke, M., Ross, L. M., Lamperti, E. D., Schulte, E., \& Schumacher, U. (2006). Atlas of anatomy : Neck and internal organs. Stuttgart, Germany: Thieme.

Schuster, M., Lohscheller, J., Kummer, P., Eysholdt, U., \& Hoppe, U. (2005). Laser projection in high-speed glottography for high- 
precision measurements of laryngeal dimensions and dynamics. European Archives of Otorhinolaryngology, 262(6), 477-481.

Shewan, R. (1979). Voice classification: An examination of methodology. The NATS Bulletin, 35, 17-27.

Shewell, C. (2009). Voice Work: Art and science in changing voices. Chichester, UK: John Wiley \& Sons.

Shipp, T. (1975). Vertical laryngeal position during continuous and discrete vocal frequency change. Journal of Speech, Language and Hearing Research, 18(4), 707.

Shipp, T. (1982). Aspects of voice production and motor control. In S. Gillner, B. Lindblom, J. Lubker \& A. Persson (Eds.), Speech motor control (pp. 105-112). New York, NY: Pergamon Press.

Shipp, T., Doherty, E. T., \& Morrissey, P. (1979). Predicting vocal frequency from selected physiologic measures. Journal of the Acoustical Society of America, 66(3), 678-684.

Shipp, T., \& Izdebski, K. (1975). Vocal frequency and vertical larynx positioning by singers and nonsingers. Journal of the Acoustical Society of America, 58(5), 1104-1106.

Shipp, T., \& McGlone, R. E. (1971). Laryngeal dynamics associated with voice frequency change. Journal of Speech, Language and Hearing Research, 14(4), 761-768.

Shipp, T., \& Morrissey, P. (1977). Physiologic adjustments for frequency change in trained and untrained voices. The Journal of The Acoustical Society Of America, 62, 476.

Singh, M., Chin, K. J., Chan, V. W. S., Wong, D. T., Prasad, G. A., \& Yu, E. (2010). Use of sonography for airway assessment: An observational study. Journal of Ultrasound in Medicine, 29(1), 79-85.

Smith, M. E., Roy, N., Stoddard, K., \& Barton, M. (2008). How does cricotracheal resection affect the female voice? Annals of Otology, Rhinology and Laryngology 117, 85-89

Snidecor, J. (1951). The pitch and duration characteristics of superior female speakers during oral reading. Journal of Speech and Hearing Research, 16, 44-52. 
Sonesson, B. (1959). Die funktionelle Anatomie des

Cricoarytaenoidgelenkes. Zeitschrift für Anatomie und

Entwicklungsgeschichte, 121(4), 292-303.

Sonesson, B. (1982). Vocal fold kinesiology. In S. Grilln, B. Lindholm, J. Lubker \& A. Persson (Eds.), (pp. 113-118). Oxford: Pergamon Press.

Sonninen, A. (1954). Is the length of the vocal cords the same at all different levels of singing? Acta Oto-Laryngologica, 118, 219-231.

Sonninen, A. (1956). The role of the external laryngeal muscles in length adjustment of the vocal cords in singing. Acta Oto-Laryngologica (Stockholm), Supplement 130, 1-102.

Sonninen, A. (1968). The external frame function in the control of pitch in the human voice. In A. Bouhuys (Ed.), Sound production in man (Vol. 155, pp. 68-90). New York Annals of New York Acadamy of Science.

Sonninen, A., Hurme, P., \& Laukkanen, A.-M. (1999). The external frame function in the control of pitch, register, and singing mode: Radiographic observations of a female singer. Journal of Voice, 13(3), 319-340.

Sonninen, A., Hurme, P., \& Vilkman, E. A. (1992). Roentgenological observations on vocal fold length-changes with special reference to register transition and open/covered voice. Logopedics Phonatrics Vocology, 17(2), 95-106.

Sorenson, D. N. (1989). A fundamental frequency investigation of children ages 6-10 years old. Journal of Communication Disorders, 22(2), 115-123.

Spalding, G. (1995). The larynx. In P. L. Williams (Ed.), Gray's anatomy (38th ed., pp. 1140). Edinburgh, UK: Churchill Livingstone.

Sprinzl, G. M., Eckel, H. E., Sittel, C., Pototschnig, C., \& Koebke, J. (1999). Morphometric measurements of the cartilaginous larynx: An anatomic correlate of laryngeal surgery. Head Neck, 21(8), 743-750.

Statistics New Zealand. (2013). Statistics New Zealand Retrieved 29

August 2013, from

http://www.stats.govt.nz/surveys and methods/methods/classificati ons-and-standards/classification-related-stats-

standards/ethnicity/definition.aspx 
Stoicheff, M. L. (1981). Speaking fundamental characteristics of nonsmoking female adults. Journal of Speech and Hearing Research, 24, 437-441.

Stone, R., \& Nuttall, A. (1974). Relative movements of the thyroid and cricoid cartilages assessed by neural stimulation in dogs. Acta OtoLaryngologica (Stockholm), 78(1-6), 135-140.

Storck, C., Gehrer, R., Fischer, C., Wolfensberger, M., Honegger, F., Friedrich, G., \& Gugatschka, M. (2011). The role of the cricothyroid joint anatomy in cricothyroid approximation surgery. Journal of Voice, 25(5), 632-637.

Storck, C., Juergens, P., Fischer, C., Haenni, O., Ebner, F., Wolfensberger, M., \& Sorantin. (2010). Three-dimensional imaging of the larynx for pre-operative planning of laryngeal framework surgery. European Archives of Otorhinolaryngology, 267, 557-563.

Strong, M., \& Vaughan, B. (1981). The morphology of the phonatory organs and their neural control. In K. Stevens \& M. Hirano (Eds.), Vocal fold physiology (pp. 13-20). Tokyo, Japan: University of Tokyo Press.

Su, M.-C., Yeh, T.-H., Tan, C.-T., Lin, C.-D., Linne, O.-C., \& Lee, S.-Y. (2002). Measurement of adult vocal fold length. Journal of Laryngology \& Otology, 116(06), 447-449.

Sundberg, J. (1974). Articulatory interpretation of the "singing formant". Journal of the Acoustical Society of America, 55, 838-844.

Sundberg, J. (1987). The science of the singing voice. Dekald, IL: Illinois University Press.

Suverkrop, B. (2013). IPA Source. Retrieved October 12, 2010, from http://www.ipasource.com/soprano

Swinscow, T. D. V., \& Campbell, M. J. (2002). Statistics at square one. London, UK: BMJ Books.

Tanabe, M., Haji, T., Honjo, I., \& Isshiki, N. (1985). Surgical treatment for androphonia: An experimental study. Folia Phoniatrica (Basel), 37(1), 15-21.

Tayama, N., Chan, R. W., Kaga, K., \& Titze, I. R. (2001). Geometric characterization of the laryngeal cartilage framework for the purpose of biomechanical modeling. Annals of Otology, Rhinology and Laryngology, 110(12), 1154-1161. 
Teles-Magalhães, L. C., Pegoraro-Krook, M. I., \& Pegoraro, R. (2000). Study of the elderly females' voice by phonetography. Journal of Voice, 14(3), 310-321.

Testut, L., \& Jacob, O. (1921). Traite d'anatomie topographique avec applications medico-chirurgicales. Paris, France: O. Doin et fils.

Thomas, J. (2004). Cricothyroid approxmation surgery. Retrieved May 26, 2011, from http://voicedoctor.net/surgery/pitch-altering-surgeries

Titze, I. R. (1981). What determines the elastic properties of the vocal folds and how important are they? Journal of Singing, 38, 30-31.

Titze, I. R. (1988). Male-female differences in the larynx. Voice Research, $44,31-32$.

Titze, I. R. (1993). Current topics in voice production mechanisms. Acta Otolaryngol (Stockh), 113, 421-427.

Titze, I. R. (1994). Principles of voice production. Englewood Cliffs, N.J.: Prentice Hall.

Titze, I. R. (1996). Why do we have a vocal ligament. Journal of Singing, $53,31-32$.

Titze, I. R. (1998a). Voice research: Five ingredients of a physiologically gifted voice. Journal of Singing, 54(3), 45-46.

Titze, I. R. (1998b). The wide pharynx. Journal of Singing, 55, 27.

Titze, I. R. (2000). Principles of voice production. Iowa City, IA: National Center for Voice and Speech.

Titze, I. R., \& Hunter, E. J. (2004). Normal vibration frequencies of the vocal ligament. Journal of the Acoustical Society of America, 115(5 Pt 1), 2264-2269.

Titze, I. R., Luschei, E. S., \& Hirano, M. (1989). Role of the thyroarytenoid muscle in regulation of fundamental frequency. Journal of Voice, 3(3), 213-224.

Tortora, G. J., \& Derrickson, B. (2006). Principles of anatomy and physiology: John Wiley \& Sons.

Traunmuller, H., \& Eriksson, A. (1994). The frequency range of the voice fundamental in the speech of male and female adults. Stockholm, 
Sweden: Institutionen för lingvistik, Stockholms universitet, S-106 91.

Tucker, H., M. (1985). Anterior commissure laryngoplasty for adjustment of vocal fold tension. Annals of Otology, Rhinology, and Laryngology, 94, 547-549.

Tucker, H. M. (1989). Laryngeal framework surgery in the management of spasmodic dysphonia. Preliminary report. Annals of Otology, Rhinology, and Laryngology, 98(1 Pt 1), 52-54.

Van Den Berg, J., \& Tan, T. S. (1959). Results of experiments with human larynxes. Practica Oto-Rhino-Laryngologica, 21, 425-450.

van Dommelen, W. A., \& Moxness, B. H. (1995). Acoustic parameters in speaker height and weight identification: Sex-specific behaviour. Language \& Speech, 38(Part 3), 267-287.

Vennard, W. (1967). Singing: The mechanism and the technique (5th ed.). New York, NY: Carl Fischer.

Verdonck-de Leeuw, I. M., \& Mahieu, H. F. (2004). Vocal aging and the impact on daily life: A longitudinal study. Journal of Voice, 18(2), 193-202.

Vilkman, E. A. (1987). An apparatus for studying the role of the cricothyroid articulation in the voice production of excised human larynges. Folia Phoniatr Logopaedica, 39(4), 169-177.

Vilkman, E. A., Pitkänen, R., \& Suominen, H. (1987). Observations on the structure and the biomechanics of the cricothyroid articulation. Acta Oto-Laryngologica, 103(1-2), 117-126.

Vilkman, E. A., Sonninen, A., Hurme, P., \& Körkkö, P. (1996). External laryngeal frame function in voice production revisited: A review. Journal of Voice, 10(1), 78-92.

Vilkman, E. A., Takalo, R., Maatta, T., Laukkanen, A.-M., Nummenranta, J., \& Lipponen, T. (1997). Ultrasonographic measurement of cricothyroid space in speech. Paper presented at the EUROSPEECH '97 5th European Conference on Speech Communication and Technology: September 22-25, Rhodes, Greece. pp.39-42.

Von Leden, H. (1977). Speech and hearing problems in the geriatric patient. Journal of the American Geriatrics Society, 25(9), 422-426. 
Washington Voice Consortium. (2013). The voice problem website: Understanding how voice is produced. Retrieved March 20, 2012, from http://www.voiceproblem.org/anatomy/understanding.php

Watson, P., \& Hixon, T. (1985). Respiratory kinematics in classical (opera) singers. Journal of Speech and Hearing Research, 28, 104-122.

Wendler, J. (1990). Vocal pitch elevation after transexualism male to female. Paper presented at the Proceedings of the Union of the European Phoniatricians, Salsomaggiore, Italy.

Wheat, M. C., \& Hudson, A. I. (1988). Spontaneous speaking fundamental frequency of 6-year-old black children. Journal of Speech and Hearing Research, 31, 723-725.

Windisch, G., Hammer, G. P., Prodinger, P. M., Friedrich, G., \& Anderhuber, F. (2010). The functional anatomy of the cricothyroid joint. Surgical \& Radiologic Anatomy, 32(2), 135-139.

Wolfe, J., Garnier, M., \& Smith, J. (2010). Voice acoutics: An introduction. Retrieved June 3, 2011, from http://www.phys.unsw.edu.au/jw/voice.html

Wolfe, V. I., Ratusnik, D. L., Smith, F. H., \& Northrop, G. (1990). Intonation and fundamental frequency in male-to-female transsexuals. Journal of Speech and Hearing Disorders, 55(1), 43.

Xue, A., \& Mueller, P. B. (1996). Speaking fundamental frequency of elderly African-American nursing home residents: Preliminary data Clinical Linguistics and Phonetics, 10, 65-70.

Yanagihara, N., \& von Leden, H. (1968). The cricothyroid muscle during phonation. Annals of Otology, Rhinology and Laryngology, 75, 9871006.

Yanagisawa, E., Estill, J., Kmucha, S. T., \& Leder, S. B. (1989). The contribution of aryepiglottic constriction to "ringing" voice quality: A videolaryngoscopic study with acoustic analysis. Journal of Voice, 3(4), 342-350.

Yang, C. Y., Palmer, A. D., Meltzer, T. R., Murray, K. D., \& Cohen, J. I. (2002). Cricothyroid approximation to elevate vocal pitch in malefemale transsexuals: Results of surgery. Annals of Otology, Rhinology and Laryngology, 111, 477-485.

Zeitels, S. M., Hillman, R. E., Desloge, R. B., \& Bunting, G. A. (1999). Cricothyroid subluxation: A new innovation for enhancing the voice 
with laryngoplastic phonosurgery. Annals of Otology, Rhinology and Laryngology, 108(12), 1126-1131.

Zenker, W., \& Zenker, A. (1960). On the regulation of vocal cord tension through externally influencing mechanisms. Folia Phoniatrica (Basel), 12, 1-36.

Zraick, R. I., Birdwell, K., \& Smith-Olinde, L. (2005). The effect of speaking sample duration on determination of habitual pitch. Journal of Voice, 19, 197-201.

Zraick, R. I., Skaggs, S. D., \& Montague, J. C. (2000). The effect of task on determination of habitual pitch. Journal of Voice, 14, 484-489. 


\section{Appendices}

\section{Appendix A: Surgical procedures for pitch modification}

The following passage describes several surgical procedures for raising and lowering voice pitch.

\section{- Cricothyroid Approximation}

Cricothyroid approximation (CTA) surgery (type IV thyroplasty) was the first procedure described to alter vocal pitch and was introduced by Isshiki in 1974 (Isshiki, et al., 1974; Isshiki, et al., 1983; Isshiki, 1989). This procedure reduces the CT space by approximating the cricoid cartilage to the lower margin of the thyroid cartilage, thereby increasing vocal fold tension (Lawrence, 2004) and elevating vocal pitch (Isshiki, 1989; Neumann, et al., 2002). The increase in tension causes thinning of the vocal folds although there is no actual decrease in total vocal fold mass and so it is still the increased tension alone that accounts for the increase in vocal pitch (Proctor, 1980; Kunachak et al., 2000). The technique of CTA effectively mimics the normal contraction of the cricothyroid muscles (Thomas, 2004) and thus raises the $F_{0}$ of the voice (Kunachak, et al., 2000; Matai et al., 2003).

CTA is the safest surgical procedure to increase vocal pitch, as it alters the position and tension of the vocal fold without incising the vocal folds (Isshiki, et al., 1983). For this reason it has become the standard procedure to raise vocal pitch (Storck, et al., 2011) especially in male to female transgender reassignments (Yang, et al., 2002). Theoretically, the procedure is reversible (Neumann, et al., 2002; Lawrence, 2004). A fibrescope may be used to allow the surgeon to visually monitor the degree of stretching of the vocal folds (Colton, et al., 2011).

The procedure has limitations, not least that the results are inconsistent (Lawrence, 2004). The cricoid and thyroid cartilages must be approximated under tension and occasionally the cartilages can rupture (Gross, 1999). Whilst the cricoid cartilage is solid enough to tolerate 
tension of sutures, the mid-portion of the thyroid cartilage is relatively thin and requires boosters (e.g. silicone) to disperse the pressure (Kitajima, et al., 1979). Furthermore, the pitch increase is sometimes insufficient, because $F_{0}$ varies with the square root of vocal cord tension (Lawrence, 2004). There are also physical limitations on how much room there is to rotate the cricoid cartilage toward the thyroid cartilage, which depends on the individual's preoperative CT space (Koufman \& Isacson, 1991; RicciMaccarini, et al., 2011). Late loosening of the sutures may occur, resulting in deterioration of $F_{0}(R E F)$. Increasing popularity and experience of the technique has resulted in technical modifications in an attempt to reduce complications (Lee, 1986; Sataloff (1997) Neumann et al., (2002); Matai, (2003); Friedrich, (2007).

\section{- Cricothyroid Subluxation}

Cricothyroid subluxation is a relatively new procedure that increases the distance between the cricoarytenoid joint and the insertion of the anterior commissure. It is a relatively adjustable procedure that increases the length and viscoelastic tension of the denervated vocal fold (Zeitels et al., 1999).

- Anterior - posterior expansion of the thyroid ala

This is done by vertically incising the thyroid ala and inserting a silicone wedge to broaden the thyroid cartilage and increase vocal fold tension (Isshiki, et al., 1983). This has been shown to be less effective than CT approximation in raising $F_{0}(R E F)$.

\section{- Anterior Commissure Laryngoplasty}

This is the second most popular surgical technique to increase $F_{0}$. This may be achieved by anterior web creation. Variants of this procedure include anterior commissure with vocal fold webbing (Donald, 1982); Wendler's glottoplasty (Wendler, 1990); endolaryngeal shortening of the vocal folds (Gross \& Fehland, 1995; Gross, 1999) and anterior commissure advancement using either a vocal ligament tightening 
technique (LeJeune, et al., 1983) or an anterior commissure laryngoplasty for adjustment of vocal fold tension (Tucker, 1985).

The principle of vocal fold webbing is to shorten the vibratory length of the vocal folds by creating an anterior commissure web. This can be achieved by de-epithelializing the anterior third of the vocal folds with a laser, suturing the corresponding margins of the vocal folds together allowing them to fuse and create a new V shaped anterior commissure. Only the posterior portions of the vocal folds are left free to vibrate. A portion of the anterior thyroid cartilage is also removed in Donald's (1982) technique and further minor modifications were made in Wendler's glottoplasty (Wendler, 1990).

Anterior commissure advancement is accomplished by advancing the anterior commissure anteriorly relative to the arytenoid cartilage. This procedure involves creating a vertical rectangular flap in the mid-portion of the anterior thyroid cartilage at the level of the vocal cords and then displacing it forwards with the vocal folds. This results in anteroposterior lengthening of the thyroid cartilage at the level of the vocal cords. Vocal fold stretching can be visually monitored by means of a fibrescope and vocal pitch monitored audibly during surgery if the procedure is performed under local anaesthesia. Unfortunately, due to the ventral shift of the thyroid cartilage the larynx may become prominent (LeJeune, (1983).

More aggressive modifications of this group of procedures have been reported (Kunachak, et al., 2000; Thomas, 2004). Both techniques are irreversible and complex with a high risk of impairing the voice but nevertheless apparently effective for long term voice change. The principle of both procedures is to shorten and increase the tension of both vocal folds. This is accomplished by excision of a segment of thyroid cartilage along with resection of the anterior third of the vocal folds and reconstruction of a new $\mathrm{V}$ anterior commissure. Both surgeries require general anaesthesia, so monitoring of pitch is not possible. 
- Laser procedures

Several different but less effective procedures for raising $F_{0}$ have been reported using a laser to reduce vocal fold mass and/or increase vocal fold tension and stiffness by scarring (Isshiki, et al., 1983). The advantage of these procedures is that they can be performed endoscopically without a neck incision. In Laser Assisted Voice Adjustment (LAVA; Orloff Technique) the laser is directed at the epithelium of the vocal folds causing scarring and stiffness and increasing vocal fold tension (Orloff et al., 2006). An alternative procedure, partial thyroarytenoid myectomy, has been claimed to be an alternative and safe method to reduce vocal fold mass (Genack et al., 1993) Abitbol, (1995).

- Scarification of vocal folds

As a variant of laser treatment, other surgical procedures have been used to raise vocal pitch by increasing vocal fold stiffness from scarring. Most result in a permanent modification of vocal fold consistency. Some of the proposed methods are: vocal fold stripping (Hirano, et al., 1969); longitudinal incisions in the vocal folds to cut the vocalis muscle (Kokawa, 1977; Saito, 1977) and scarification (Tanabe et al., 1985).

- Injection laryngoplasty

The vocal folds can be injected under general or local anaesthetic either directly or transcutaneously through the cricothyroid membrane with a variety of agents including corticosteroids (Isshiki, et al., 1983), teflon or collagen (Mahieu \& Schutte, 1989). This produces atrophy, which decreases vocal fold mass at the site of injection. The theory is that thinner vocal folds will vibrate at a higher pitch thus increasing SFF.

- Relaxation laryngoplasty

As $F_{0}$ decreases, the vocal folds elongate, are less tense and have a greater vibrating mass. According to the European Laryngological Society, techniques that attempt to surgically lower $F_{0}$ can be grouped under the heading of relaxation laryngoplasty (Friedrich, et al., 2007) (Table A1). 
Table A16: Relaxation laryngoplasty: surgical procedure

\begin{tabular}{|l|l|}
\hline \multicolumn{2}{|c|}{ Relaxation Laryngoplasty } \\
\hline Shortening thyroplasty & Type III thyroplasty \\
\hline Lateral approach & Anterior commissure retrusion \\
\hline Medial approach & Relaxation of both vocal folds \\
\hline Combination laryngoplasty & $\begin{array}{l}\text { Relaxation and increased mass of one vocal } \\
\text { fold }\end{array}$ \\
\hline Bilateral relaxation laryngoplasty \\
\hline laryngoplasty
\end{tabular}

The major aim of relaxation laryngoplasty is to shorten the distance between the anteroposterior vocal fold attachments, reducing tension in the vocal folds and decreasing pitch (Isshiki, 1977). The technique can be used for inappropriately high pitch voices or pathologically tight or stiff vocal folds (Friedrich, et al., 2007).

There are two main types of shortening thyroplasties, one using a lateral approach (thyroplasty III) and one those via a medial approach (anterior commissure retrusion). They can be used separately or in combination.

(i) Thyroplasty III

The classic type III thyroplasty, or anteroposterior shortening of the thyroid ala by anterior vertical strip excision of the thyroid cartilage, was introduced by Isshiki (Isshiki, et al., 1974; Isshiki, et al., 1983; Isshiki, 1989). The procedure causes retrusion of the middle portion of the thyroid cartilage thereby reducing the length of the vocal folds (Balasubramanian, 2010).

(ii) Anterior commissure retrusion

In principle, a vertical cartilage flap in the mid-portion of the thyroid cartilage and the anterior commissure are pushed posteriorly, closer to the arytenoid cartilages, and secured (Niimi et al.,1991; (Kocak et al., 2008; Balasubramanian, 2010). This is a successful treatment option for lowering vocal pitch (Remacle et al., 2010) and can be performed under 
local anaesthesia, allowing audible monitoring of voice change (Tucker, 1989; Isshiki et al., 2001).

- Cricothyroid resection

Cricothyroid resection has been shown to lower pitch and pitch range in women (Smith, et al., 2008). The vocal function may be affected by disrupting the attachment of the cricothyroid muscles or disturbing the rocking and sliding motion at the cricothyroid joint (Smith, et al., 2008). 


\section{Appendix B: Voice types}

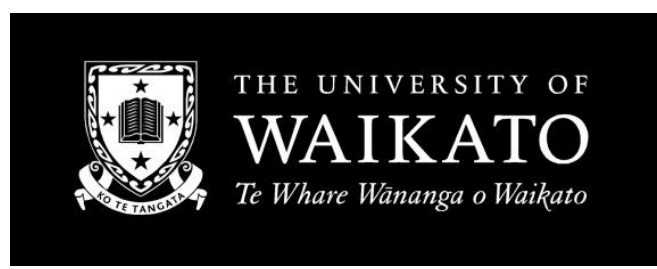

\section{Voice Types}

\section{Relationship between the Cricothyroid Space and Vocal Range.}

\section{Coloratura Soprano: $\left(C^{4}-F^{6}\right)$}

A very flexible, bright voice with an extended top range. The ability to sing extremely high notes, fast coloratura passages and a bright sound.

Typical roles:

Zerbinetta (Adriadne auf Naxos), Norina (Pasquale), Olympia (Hoffmann), Fiordiligi (Cosi), Rosalinda (Fledermaus), Violetta (Traviata), Donna Elvira (Giovanni), Nedda (Pagliacci), Lucia (Lucia di Lammermoor), Countess (Figaro), Queen of the Night (Magic Flute)

\section{Soubrette: $\left(C^{4}-C^{6}\right)$}

A mellow supple voice, delicate physical appearance, an excellent actress.

Much of the singing lies in middle voice, a warm soprano.

Typical roles:

Adele (Fledermaus), Despina (Cosi), Lauretta (Gianni Schicchi), Musetta (Boheme), Susanna (Figaro), Zerlina (Giovanni), Marzellina (Fidelio) 


\section{Lyric Soprano: $\left(C^{4}-C^{6}\right)$}

A supple voice with a beautiful mellow quality and a noble line.

Lyric means ability to sustain lines with more voice and full legato line.

Typical roles:

Mimi (Boheme), Countess (Figaro), Antonia (Hoffmann), Pamina (Magic Flute), Michaela (Carmen), Lauretta (Gianni Schicchi),Rusalka (Rusalka), Liu (Turandot), Margarethe (Faust)

\section{Spinto: $\left(C^{4}-C^{6}\right)$}

A metallic voice with good line and power, capable of bringing moments of dramatic intensity.

Typical roles:

Donna Anna (Giovanni), Elsa (Lohengrin), Desdemona (Othello), Leonora (Der Troubadour), Tatajana (Eugene Onegin), Elizabeth (Tannhauser)

\section{Dramatic Soprano: $\left(B^{3}-C^{6}\right)$}

A large, heavy and projecting instrument with well-developed middle and lower registers.

Typical roles:

Leonora (Fidelio), Aida (Aida), Elizabeth (Don Carlos), Tosca (Tosca), Ariadne (Ariadne auf Naxos), Brunnhilde (Die Walkure) (Die Gotterdammerung), Elektra (Elektra), Isolde (Tristan und Isolde), Turandot (Turandot) 


\section{Lyric Mezzo-soprano: $\left(A^{3}-A^{5}\right)$}

A flexible, expressive voice, an excellent actress.

Mezzo counterpart of the soubrette. Quite often a pants role.

Voice is a flexible, mellow instrument lacking the metallic ring and broad legato line.

Typical roles:

Rosina (Seville), Dorabella (Cosi), Cherubino (Figaro), Orlofsky (Fledermaus), Cenerentola (Cenerentola), Siebel (Faust), Sextus (La Clemenza di Tito), Charlotte (Werther)

\section{Dramatic Mezzo-soprano: $\left(A^{3}-B^{5}\right)$}

A flexible, metallic voice with a well-developed top and bottom register, a dark colour that often develops with age, and capable of dramatic intensity.

Typical roles:

Eboli (Don Carlos), Leonora (La Favorita), Waltraute (Gotterdammerung), Orturd (Lohengrin), Octavin (Rosenkavalier), Dalila (Samson und Dalila), Carmen (Carmen)

\section{Contralto: $\left(E^{3}-G^{5}\right)$}

A full rich voice with an extended bottom range.

This is rather a special category and an important one. It contains many roles demanding an intensive low voice and a mature appearance.

Typical roles:

Ulrica (Ein Maskenball), Erda (Das Rheingold), Azucenza (Der Troubadour), Orpheus (Orpheus und Eurudike) 


\section{Appendix C: Standard 25 Fach types}

Female Fach voice types

\begin{tabular}{|c|c|c|c|}
\hline Voice type & English & German & Characteristics \\
\hline \multirow[t]{7}{*}{ Soprano } & Soubrette & Spielsopran & Young, light, bright \\
\hline & Lyric coloratura & $\begin{array}{c}\text { Lyrischer } \\
\text { Koloratursopran }\end{array}$ & $\begin{array}{l}\text { High, bright, } \\
\text { flexible }\end{array}$ \\
\hline & $\begin{array}{l}\text { Dramatic } \\
\text { coloratura }\end{array}$ & $\begin{array}{c}\text { Dramatischer } \\
\text { Koloratursopran }\end{array}$ & High, dark, flexible \\
\hline & Lyric soprano & Lyrischer Sopran & Warm, legatto, full \\
\hline & $\begin{array}{l}\text { Character } \\
\text { soprano }\end{array}$ & Charaktersopran & $\begin{array}{c}\text { Bright, metallic, } \\
\text { theatrical }\end{array}$ \\
\hline & $\begin{array}{l}\text { Spinto/ Young } \\
\text { dramatic } \\
\text { soprano }\end{array}$ & $\begin{array}{l}\text { Jugendlich- } \\
\text { dramatischer } \\
\text { Sopran }\end{array}$ & $\begin{array}{l}\text { Powerful, young, } \\
\text { full }\end{array}$ \\
\hline & Dramatic & $\begin{array}{c}\text { Dramatischer } \\
\text { Sopran }\end{array}$ & $\begin{array}{l}\text { Powerful, dark, } \\
\text { rich }\end{array}$ \\
\hline \multirow[t]{3}{*}{$\begin{array}{l}\text { Mezzo- } \\
\text { soprano }\end{array}$} & Coloratura & $\begin{array}{c}\text { Coloratura } \\
\text { Mezzo-Soprano }\end{array}$ & Agile, rich, bright \\
\hline & Lyric & $\begin{array}{c}\text { Lyrischer } \\
\text { Mezzosopran }\end{array}$ & $\begin{array}{c}\text { Strong, flexible, } \\
\text { lachrymose }\end{array}$ \\
\hline & Dramatic & $\begin{array}{l}\text { Dramatischer } \\
\text { Mezzosopran }\end{array}$ & $\begin{array}{l}\text { Rich, powerful, } \\
\text { imposing }\end{array}$ \\
\hline \multirow[t]{2}{*}{ Alto } & Dramatic & Dramatischer Alt & $\begin{array}{c}\text { Powerful, full, } \\
\text { metallic }\end{array}$ \\
\hline & Low & Tiefer Alt & Low, full, warm \\
\hline
\end{tabular}


Male Fach voice types

\begin{tabular}{|c|c|c|c|}
\hline Voice type & English & German & Characteristics \\
\hline \multirow[t]{5}{*}{ Tenor } & Countertenor & Contratenor & $\begin{array}{l}\text { High, agile, } \\
\text { powerful }\end{array}$ \\
\hline & Lyric Tenor & Lyrischer Tenor & $\begin{array}{l}\text { Soft, warm, } \\
\text { flexible }\end{array}$ \\
\hline & Acting Tenor & Spieltenor & $\begin{array}{c}\text { Flexible, theatrical, } \\
\text { light }\end{array}$ \\
\hline & Dramatic Tenor & Heldentenor & Full, low, stamina \\
\hline & Character Tenor & Charaktertenor & $\begin{array}{l}\text { Bright, powerful, } \\
\text { theatrical }\end{array}$ \\
\hline \multirow[t]{4}{*}{ Baritone } & Lyric Baritone & Lyrischer Bariton & $\begin{array}{l}\text { Smooth, flexible, } \\
\text { sweet }\end{array}$ \\
\hline & $\begin{array}{l}\text { Cavalier } \\
\text { Baritone }\end{array}$ & Kavalierbariton & $\begin{array}{c}\text { Brilliant, warm, } \\
\text { agile }\end{array}$ \\
\hline & $\begin{array}{l}\text { Character } \\
\text { Baritone }\end{array}$ & Charakterbariton & $\begin{array}{c}\text { Flexible, powerful, } \\
\text { theatrical }\end{array}$ \\
\hline & $\begin{array}{l}\text { Dramatic } \\
\text { Baritone }\end{array}$ & Heldenbariton & $\begin{array}{l}\text { Powerful, full, } \\
\text { imposing }\end{array}$ \\
\hline \multirow[t]{4}{*}{ Bass } & Character Bass & Charakterbass & Full, rich, stamina \\
\hline & Acting Bass & Spielbass & Flexible, agile, rich \\
\hline & $\begin{array}{c}\text { Heavy Acting } \\
\text { Bass }\end{array}$ & $\begin{array}{l}\text { Schwerer } \\
\text { Spielbass }\end{array}$ & Full, rich, imposing \\
\hline & Serious Bass & Seriöser Bass & $\begin{array}{l}\text { Mature, rich, } \\
\text { powerful }\end{array}$ \\
\hline
\end{tabular}




\section{Appendix D: Cadaver studies on the cricothyroid joint}

The anatomical and biomechanical features of the cricothyroid joint (CTJ) have been studied by several investigators (Mayet \& Mundnich, 1958; Maue \& Dickson, 1971; Vilkman, 1987; Vilkman, et al., 1987; Berry et al., 2003; Hammer, et al., 2010; Windisch, et al., 2010; Storck, et al., 2011). At the CTJ gliding movements occur in an anteroposterior (horizontal) and craniocaudal (vertical) direction as well as rotational movements (Figure 1.1).(Mayet \& Mundnich, 1958; Zenker \& Zenker, 1960; Minnigerode, 1967; Maue \& Dickson, 1971; Vilkman, et al., 1987; Filho, et al., 2005; Hammer, et al., 2010; Windisch, et al., 2010). Recent studies on the morphological and functional anatomy of the CTJ have shown that the anatomy of the joint directly influences the range of movement and therefore the extent of vocal fold lengthening (Windisch, et al., 2010; Storck, et al., 2011).

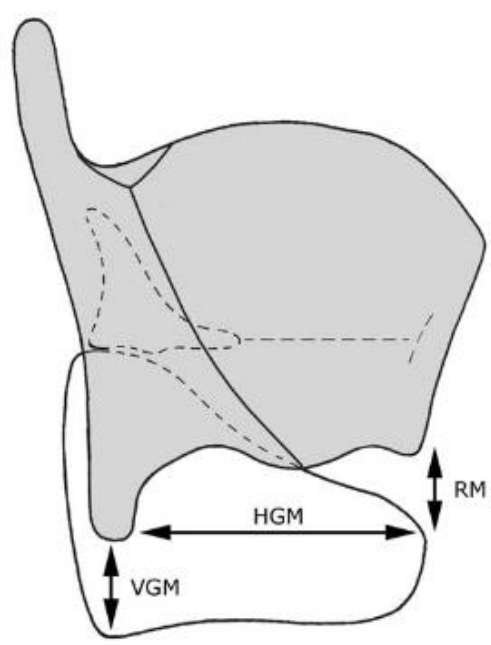

Figure A12.1: Drawing of a larynx showing movements of the thyroid cartilage secondary to movements at the CTJ. RM = rotation, $\mathrm{HGM}=$ horizontal gliding and VGM = vertical gliding motion (Windisch, et al., 2010; used with permisssion from Elsevier) 


\section{Types of CTJs}

Through the studies of Maue and Dickson (1971) it has been generally agreed that there are three types of CTJ, categorised according to their articular surface Figure 1.2) (Maue \& Dickson, 1971; Windisch, et al., 2010):

- Type A describes a well-defined joint surface on the cricoid with tight capsular ligaments and a small concavity directed from posterosuperior to anteroinferior position. This is the commonest (55-60\%).

- Type B has a relatively flat joint surface, with or without a tiny protuberance, and only a thin capsular connection. This type constitutes about $20 \%$.

- Type C shows no definable joint surface on the cricoid and only a tenuous connection by connective tissue (approximately 22\%).
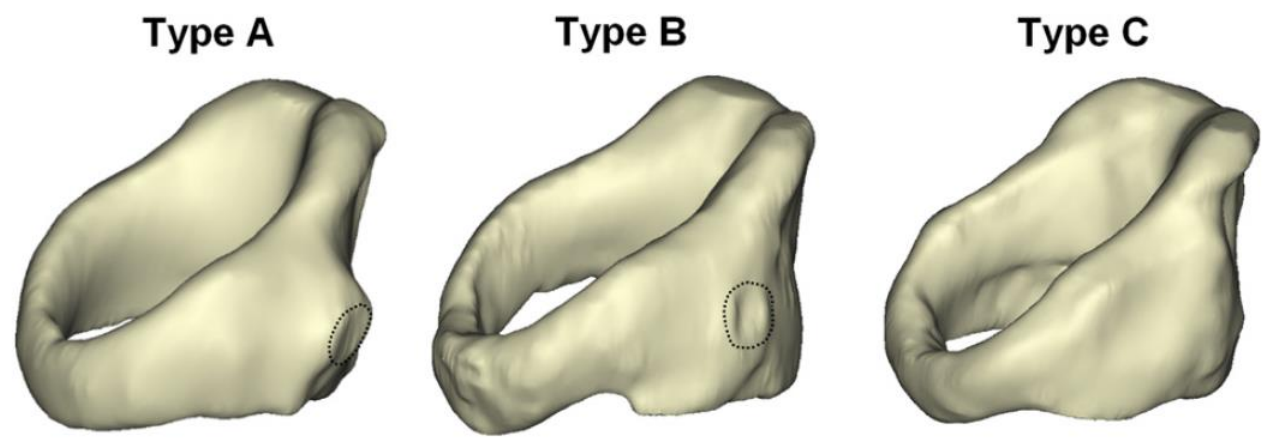

Figure A12.2: CT joints according to Maue and Dickson (1971). Oblique view of three cricoid cartilages: Type A shows a well-defined joint surface; Type B shows a flat joint surface, with only a tiny protuberance; Type $C$ shows no definable joint surface (Storck, et al., 2011; used with permission from Elsevier)

Both the stability and mobility of the CTJ depends upon the configuration of its articular facets, the joint capsule, and the extra capsular ligaments (lateral and posterior ceratocricoid ligaments) (Maue \& Dickson, 1971; Vilkman, et al., 1987; Hammer, et al., 2010). There is a highly significant difference in mobility between type $A$ and the other two. Type A with its 
tight joint capsule, tight lateral ceratocricoid ligament, and well defined joint surface is very stable with restricted horizontal and vertical gliding movements (Hammer, et al., 2010). The other types are more mobile, with type C being the most mobile (Windisch, et al., 2010). Vilkman (1987) found that the capsule contained both type I and type III collagen fibres as well as prominent elastin fibres. The collagen content of the lateral ceratocricoid ligament was negatively correlated with the degree of gliding movement.

\section{Rotational axis of the CTJ}

The traditional view is that the inferior cornu of the thyroid cartilage rotates on the cricoid cartilage about a transverse axis that runs through both CTJs (Neumann \& Welzel, 2004; Windisch, et al., 2010). Through a vector geometrical analysis, Storck (2011) demonstrated that in some cases the effective rotational axis ran considerably above the CTJ, depending on whether synchronous gliding movements were occurring. This author described three positions on the cricoid where the effective rotational axis was located: the lower third of the cricoid; the middle third of the cricoid; or the upper third of the cricoid. When the effective rotational axis was in the lower third of the cricoid close to the previously assumed axis of rotation through the CTJs then there was true rotation at the CTJ. When the effective rotational axis was located above the CTJ, then rotation and gliding occurred at the joint, equivalent to an anterior shift of the axis described by others (Figure 1.3) (Minnigerode, 1967; Maue \& Dickson, 1971; Vilkman, et al., 1987; Filho, et al., 2005; Hammer, et al., 2010; Storck et al., 2010; Windisch, et al., 2010).

Storck also found a clear correlation between the location of the effective rotational axis and the type of CTJ. Where the effective rotational axis ran through the lower third of the cricoid, individuals had a well-defined type A joint. Where the effective rotational axis ran through the middle third of the cricoid a type $B$ joint was evident. Finally, when the effective rotational axis ran through the upper third of the cricoid, a type $\mathrm{C}$ joint was found. 

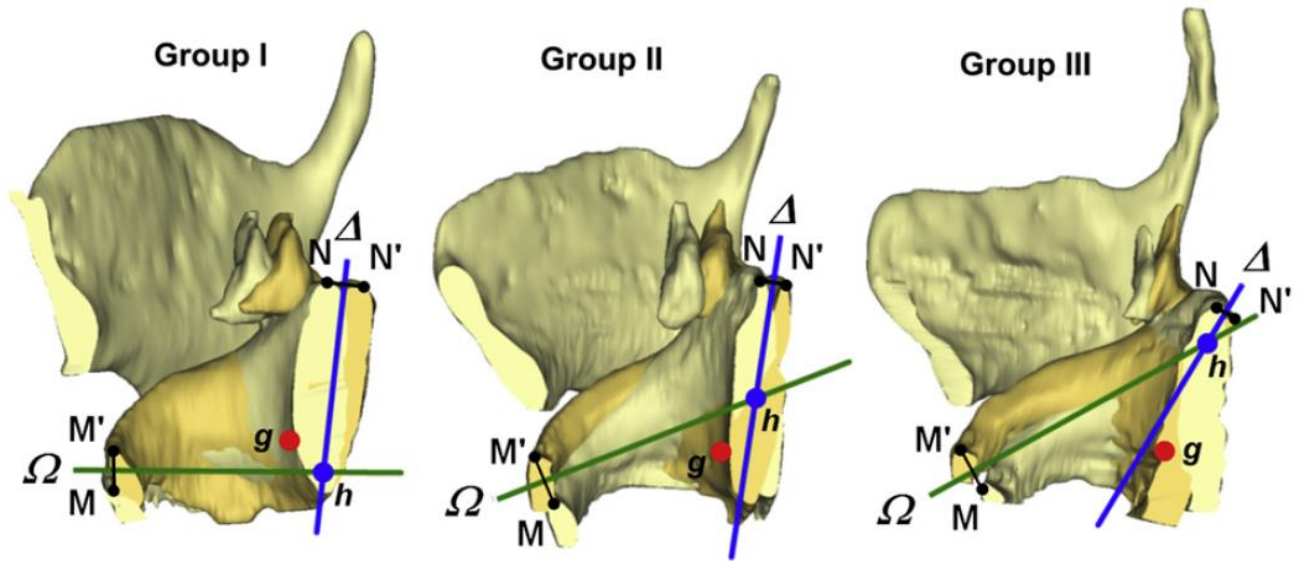

Figure A12.3: Different locations of the effective rotational axis of the CTJ. Medial view of the right hemilarynx. $g=$ traditional presumed rotational axis running through both CT joints. Group I: Effective rotational axis (h) located in lower third of cricoid cartilage; Group II: Effective rotational axis (h) located above the CTJ in middle third of cricoid cartilage; Group III: Effective rotational axis (h) located above the CTJ in upper third of cricoid cartilage (Storck, et al., 2011; used with permission from Elsevier)

When rotation occurs at the CTJ the thyroid cartilage can tilt forward causing elongation of the vocal folds. The extent of vocal fold elongation depends on the location of the effective rotational axis. Rotation around a low axis, as in joint type $A$, leads to a considerably greater vocal fold elongation than rotation around a higher axis, as in joint types $B$ and $C$. Thus, there is a clear correlation between CTJ anatomy and the degree of vocal fold lengthening. 


\section{Appendix E: Reading passage}

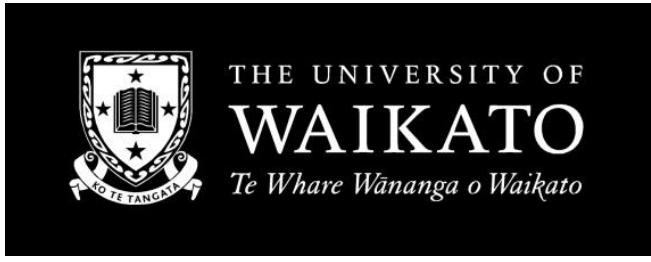

\section{Reading Passage}

\section{Relationship between the cricothyroid space and vocal range}

- Please read the following three lines in your normal speaking voice.

- Please start when I say "go".

- After each line please pause until I say "go".

1. Singing is the act of producing musical sounds with the voice.

2. The sound of each individual's singing voice is entirely unique.

3. Voice classification is the process by which human singing voices are evaluated. 


\section{Appendix F: Ethical approval, information sheet and consent form}

26 August 2010

Dear Beverley

Application for Ethical Approval: FS2010-33 “Does the Cricothyroid Space Determine Vocal Pitch Height?"

Thank you for submitting a revised Application for Ethical Approval in response to my letter of 1 August. Many thanks for the thorough and careful job you have done in responding to each point. I am pleased you have been able to clarify the issue of application to Northern $Y$ - thank you for handing me today a copy of the email from Claire Lindsay, Ethics Committees, Ministry of Health, stating that ethical approval for your project is not required under their guidelines.

I emailed you on 25 August about the statement in your Information Sheet about ACC coverage - you agreed when talking to me today that you will delete that statement.

This letter is to provide formal ethical approval for your DMA project.

I would be grateful if you would arrange for a hard copy of your revised Application to be signed by yourself and your chief supervisor and to be given to the Committee's secretary, Diane Kenning (Room J3.20) so that it can be placed in the files.

With best wishes,

John Paterson

Chair

FASS Human Research Ethics Committee 


\section{Information Sheet}

\section{Relationship between the Cricothyroid Space and Vocal Range.}

You are invited to take part in a research study that is using ultrasound to measure the cartilages at the front of your neck (the cricoid and thyroid cartilages) and the space between them (cricothyroid space) to see if these features limit the range of your singing voice. Your participation is entirely voluntary. Please take as much time as you need to consider whether you wish to take part and to discuss this with family, whānau, and friends.

\section{What is the purpose of this study?}

The cricothyroid space lies between two cartilages at the front of your neck. There is now growing evidence that the CT space is an important factor in determining vocal pitch. Although there is some ability to alter the functionality of a voice through vocal training, FACH type is mostly determined by inherited characteristics of your larynx. I would like to explore the concept that a female singer's laryngeal anatomy helps determine their $\mathrm{FACH}$. Research indicates that laryngeal dimensions have a bearing on the range and timbre of an individual voice i.e. whether a singer is an alto, a soprano or a coloratura soprano. Regardless of voice category though, every voice is bound by an ultimate pitch at either end of its negotiable scale. Even with vocal training and exercises that develop all registers in a singer allowing them to make full use of their entire range, every singer has an upper range limit.

If we were able to determine that the cricothyroid space does indeed determine vocal pitch height, this could be useful in helping to indicate a person's true FACH. Ultrasound is a painless and safe way of seeing what's beneath the skin, and is used regularly to scan babies before birth and to diagnose internal problems. Studies have shown that the cricothyroid space can be reliably measured using ultrasound.

\section{Am I eligible to take part in this study?}

You have been invited to take part in this study because you are over 18 years of age, have no known thyroid disorder, do not currently have a 
cough or cold, and have never had vocal cord nodules. We are hoping to recruit up to 40 participants for this study.

\section{What will be involved if I agree to take part?}

You will first be asked to confirm that you have never had any thyroid disorder or vocal cord nodules. We will then need to record your age (in years and months), ethnicity, measure your weight, height and neck dimensions. You will be asked to speak into a microphone attached to a computer to record the pitch of your speaking voice, while reading a passage of script. Your vocal range will be tested through a series of vocal exercises. After that, you will be asked to sit and a clear hypoallergenic gel will be applied to your skin over the front of your neck. A smooth plastic ultrasound probe will be gently placed on the skin at this site. This will not cause any discomfort.

- Who will do the scans? A qualified female ultrasound technician with more than 20 years of clinical experience will do the scan in a private room.

- Who will collect the other data? The researcher will collect the consent forms, record your age, ethnicity, measure your weight, height, neck dimensions, pitch and range of voice.

- Where? The Ultrasound Department, $4^{\text {th }}$ Floor Waiora Building, Waikato Hospital, Pembroke Street.

- When? Any Monday - Friday from 5 pm onwards, Saturday or Sunday any time, in the months September to December 2010 inclusive. Let the researcher know when is convenient for you and she will endeavour to accommodate your request.

- How long will it take? Scans will take up to 15 minutes to complete. Your whole visit may last up to 30 minutes.

- Will I be compensated? Prior to leaving the hospital, you will be offered a petrol voucher to the value of $\$ 20$. This is to reimburse you for travel costs and any parking charges you may incur. This will be offered to you even if at some point during the testing, you wish to withdraw from the study.

We are extremely unlikely to find any abnormality on the ultrasound scan but, if we did, we would tell you of this finding and suggest that you inform your GP.

\section{Can I withdraw from the study at any time?}

Yes, you can change your mind and withdraw without having to give a reason. There are no consequences if you decide to withdraw. But please note that once I have started analysing your data you can no longer withdraw. 


\section{Are there any risks from taking part in this study? No}

\section{Are there any benefits from taking part in the study?}

The only real benefit is finding out if how high we can sing is determined by the natural dimensions of our cricoid and thyroid cartilages and the intervening cricothyroid space. This could be useful when female singers can't work out what FACH they are, are unhappy with their current $\mathrm{FACH}$, or feel they are singing the wrong repertoire.

\section{Will the information in the study be confidential?}

During and after the study, the information you provide will be kept confidential and stored securely at the University. Only the principal investigator and ultrasound technician will have access to your data during and after the study. Your name will be removed to make the ultrasound images and other data anonymous. Only anonymised data will be discussed with the researcher's supervisors. The researcher hopes to have the results of this study presented as part of their Doctor of Musical Arts, but no material which could personally identify you will be used in any report. Anonymised summary findings may also be published in a scientific journal. Anonymous data from the study will be stored for five years.

\section{Will I get to see the results of this study?}

There will be a delay of up to three years between doing the scans and fully analysing the results. At the end of the study you will be sent a short summary of the findings if you request this on the consent form. In the meantime, you are welcome to contact the researcher for information about the results of the study when they become available.

This research project has been approved by the Human Research Ethics Committee of the Faculty of Arts and Social Sciences. Any questions about the ethical conduct of this research may be sent to the Secretary of the Committee, email fass-ethics@waikato.ac.nz, postal address, Faculty of Arts and Social Sciences, Te Kura Kete Aronui, University of Waikato, Te Whare Wananga o Waikato, Private Bag 3105, Hamilton 3240.

Thank you for considering taking part in this study.

Please feel free to contact the researcher or her supervisors if you have any questions.

Researcher: Beverley Pullon (cullanna@clear.net.nz) Telephone (07) 8563437 or 0274257235 
Supervisors: Professor Mark Stringer mark.stringer@anatomy.otago.ac.nz (03) 4795992

Martin Lodge mlodge@waikato.ac.nz

David Griffiths GRIFFMUS@waikato.ac.nz 


\section{Consent form}

THE UNIVERSITY OF

\section{Consent Form}

\section{Relationship between the Cricothyroid Space and Vocal Range.}

I have read and understood the Information Sheet for volunteers taking part in this ultrasound study of measuring the cricoid and thyroid cartilages in the neck and the distance between them.

I have had the opportunity to ask questions and discuss the study and I am satisfied with the answers to my questions.

I have had the opportunity to use whānau support or a friend/family member to help me ask questions and understand the study.

I have received enough information about this study and have had time to consider whether to take part.

I understand that taking part in this study is entirely voluntary (my choice) and that I am free to withdraw from the study up to the point when the scans are completed. I do not have to give a reason and this would not affect my future healthcare.

I understand that my participation in this study is confidential and that no material that could identify me personally will be used in any reports on this study. I understand that non-identifying data collected from me by the researcher will be stored on a secure computer at the University of Waikato and may be published.

I can contact either the researcher or her supervisors if I have any questions about the study. 
I am aware that I can contact the study investigator for a copy of the results of this study when they become available but that this may be up to three years from now.

I wish to receive a short summary of the findings. YES NO (Please circle your choice)

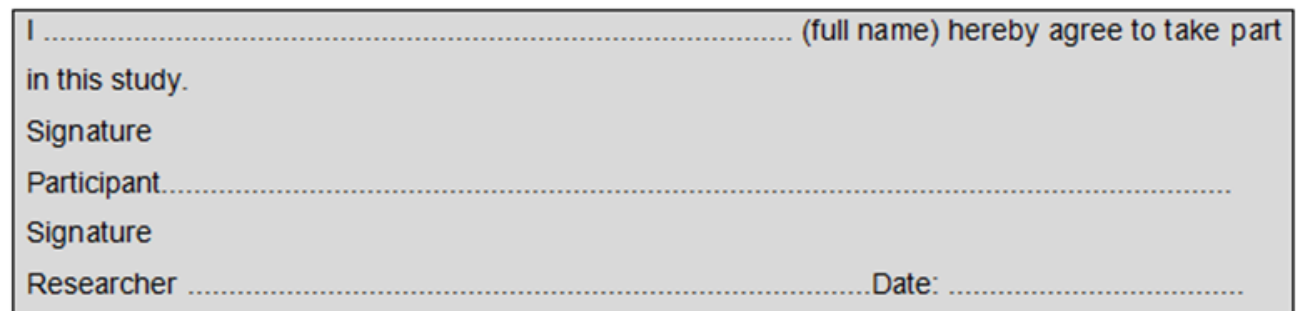

This research project has been approved by the University of Waikato Human Research Ethics Committee of the Faculty of Arts and Social Sciences. Any questions about the ethical conduct of this research may be sent to the Secretary of the Committee, email fass-ethics@waikato.ac.nz, postal address, Faculty of Arts and Social Sciences, Te Kura Kete Aronui, University of Waikato, Te Whare Wananga o Waikato, Private Bag 3105, Hamilton 3240. Any issues you raise will be treated in confidence and investigated and you will be informed of the outcome.

Please feel free to contact the researcher or her supervisors if you have any questions.

Researcher: Beverley Pullon (cullanna@clear.net.nz) Telephone (07)856 3437 or 0274257235

Supervisors: Professor Mark Stringer mark.stringer@anatomy.otago.ac.nz (03) 4795992

Martin Lodge mlodge@waikato.ac.nz

David Griffiths GRIFFMUS@waikato.ac.nz 


\section{Appendix G: Summary of anthropometric data divided into voice types soprano and mezzo-soprano}

\begin{tabular}{|c|c|c|c|c|}
\hline \multicolumn{5}{|c|}{ Sopranos } \\
\hline Parameter & $\begin{array}{c}\text { Mean } \\
\mathbf{( n = 3 2 )}\end{array}$ & $\begin{array}{c}\text { SD } \\
(\mathbf{n = 3 2})\end{array}$ & SE mean & $\begin{array}{c}\text { Range } \\
\mathbf{( n = 3 2 )}\end{array}$ \\
\hline Age (years) & 36.3 & 14.4 & 2.5 & $18-63$ \\
\hline Height (cm) & 164.9 & 6.6 & 1.2 & $151.3-177$ \\
\hline Weight (kg) & 71.9 & 18.7 & 3.3 & $47.4-139.8$ \\
\hline BMI (kg/m $\left.{ }^{2}\right)$ & 26.4 & 6.0 & 1.1 & $19.3-44.6$ \\
\hline Neck circumference (cm) & 34.0 & 3.4 & 0.6 & $30.0-45.5$ \\
\hline Neck length (cm) & 11.9 & 1.7 & 0.3 & $9.0-15.4$ \\
\hline $\begin{array}{c}\text { Cricoid cartilage arch height in } \\
\text { midline (mm) }\end{array}$ & 5.6 & 0.7 & 0.1 & $4.4-7.2$ \\
\hline $\begin{array}{c}\text { Thyroid cartilage height in } \\
\text { midline (mm) }\end{array}$ & 12.2 & 2.0 & 0.4 & $8.3-15.3$ \\
\hline Cricothyroid space (mm) & 10.4 & 1.4 & 0. & $7.3-12.4$ \\
\hline $\begin{array}{c}\text { Combined height of thyroid and } \\
\text { cricoid cartilages \& cricothyroid } \\
\text { space in midline (mm) }\end{array}$ & 28.2 & 2.9 & 0.5 & $21.8-33.7$ \\
\hline
\end{tabular}

\begin{tabular}{|c|c|c|c|c|}
\hline \multicolumn{5}{|c|}{ Mezzo-sopranos } \\
\hline Parameter & $\begin{array}{c}\text { Mean } \\
(\mathbf{n = 1 1 )}\end{array}$ & $\begin{array}{c}\text { SD } \\
(\mathbf{n = 1 1 )}\end{array}$ & SE mean & $\begin{array}{c}\text { Range } \\
(\mathbf{n = 1 1})\end{array}$ \\
\hline Age (years) & 43.2 & 13.4 & 4.0 & $21-62$ \\
\hline Height (cm) & 168.2 & 6.8 & 2.04 & $155.7-176.5$ \\
\hline Weight (kg) & 76.05 & 17.0 & 5.1 & $58.2-107.5$ \\
\hline BMI (kg/m²) & 26.9 & 5.6 & 1.7 & $19.5-38.5$ \\
\hline Neck circumference (cm) & 34.0 & 2.7 & 0.8 & $30.5-39.8$ \\
\hline $\begin{array}{c}\text { Neck length (cm) } \\
\text { Cricoid cartilage arch height in } \\
\text { midline (mm) }\end{array}$ & 12.7 & 1.0 & 0.3 & $11.5-14.5$ \\
\hline $\begin{array}{c}\text { Thyroid cartilage height in } \\
\text { midline (mm) }\end{array}$ & 12.0 & 2.2 & 0.7 & 9.4 \\
\hline Cricothyroid space (mm) & 11.6 & 1.1 & 0.3 & $10.5-14.0$ \\
\hline $\begin{array}{c}\text { Combined height of thyroid and } \\
\text { cricoid cartilages \& cricothyroid } \\
\text { space in midline (mm) }\end{array}$ & 29.5 & 3.1 & 1.0 & $25.2-35.0$ \\
\hline
\end{tabular}


Appendix H: Summary of acoustic data divided into voice types soprano and mezzo-soprano

\begin{tabular}{|c|c|c|c|c|}
\hline \multicolumn{5}{|c|}{ Sopranos } \\
\hline Parameter & $\begin{array}{l}\text { Mean } \\
(n=32)\end{array}$ & $\begin{array}{c}\text { SD } \\
(n=32)\end{array}$ & SE mean & $\begin{array}{l}\text { Range } \\
(\mathrm{n}=32)\end{array}$ \\
\hline $\begin{array}{c}\text { Speaking fundamental } \\
\text { frequency } \\
\operatorname{SFF}(\mathrm{Hz})\end{array}$ & 207 & 23.1 & 4.1 & $152-243$ \\
\hline $\begin{array}{l}\text { Lowest performance note } \\
\text { (pitch) }\end{array}$ & G3 & 2.1 & 0.4 & D3-A\#3 \\
\hline $\begin{array}{l}\text { Highest performance note } \\
\text { (pitch) }\end{array}$ & D6 & 2.0 & 0.4 & A\#5-F6 \\
\hline $\begin{array}{l}\text { Performance vocal range } \\
\text { (semitones) }\end{array}$ & 31 & 3.2 & 0.6 & $24-39$ \\
\hline $\begin{array}{l}\text { Lowest practice note } \\
\text { (pitch) }\end{array}$ & E3 & 2.5 & 0.4 & A\#2-G3 \\
\hline $\begin{array}{l}\text { Highest practice note } \\
\text { (pitch) }\end{array}$ & F6 & 2.3 & 0.4 & C6-A6 \\
\hline Practice vocal range (semitones) & 37 & 3.6 & 0.6 & $29-46$ \\
\hline \multicolumn{5}{|c|}{ Mezzo-sopranos } \\
\hline Parameter & $\begin{array}{l}\text { Mean } \\
(n=11)\end{array}$ & $\begin{array}{c}\text { SD } \\
(n=11)\end{array}$ & SE mean & $\begin{array}{l}\text { Range } \\
\text { ( } n=11)\end{array}$ \\
\hline $\begin{array}{l}\text { Speaking fundamental } \\
\text { frequency } \\
\text { SFF }(\mathrm{Hz})\end{array}$ & 180 & 26.3 & 7.9 & $152-240$ \\
\hline $\begin{array}{l}\text { Lowest performance note } \\
\text { (pitch) }\end{array}$ & E3 & 3.0 & 0.9 & C3-G3 \\
\hline $\begin{array}{l}\text { Highest performance note } \\
\text { (pitch) }\end{array}$ & A\#5 & 1.9 & 0.6 & G\#5-D6 \\
\hline $\begin{array}{l}\text { Performance vocal range } \\
\text { (semitones) }\end{array}$ & 30 & 3.2 & 1.0 & $25-36$ \\
\hline $\begin{array}{l}\text { Lowest practice note } \\
\text { (pitch) }\end{array}$ & $\mathrm{C \# 3}$ & 3.1 & 0.9 & G2-F3 \\
\hline $\begin{array}{l}\text { Highest practice note } \\
\text { (pitch) }\end{array}$ & $\mathrm{C \# 6}$ & 2.2 & 0.7 & A\#5-F6 \\
\hline Practice vocal range (semitones) & 36 & 4.2 & 1.3 & $31-45$ \\
\hline
\end{tabular}




\section{Appendix I: Data comparing age to SFF}

\begin{tabular}{|c|c|c|c|}
\hline Age (years) & SFF (Hz) & Age (years) & SFF (Hz) \\
\hline 18 & 167 & 40 & 159 \\
\hline 19 & 203 & 40 & 152 Lowest SFF \\
\hline 20 & 207 & 41 & 229 \\
\hline 21 & 229 & 45 & 240 \\
\hline 21 & 207 & 48 & 218 \\
\hline 21 & 196 & 49 & 243 Highest SFF \\
\hline 21 & 239 & 49 & 159 \\
\hline 22 & 218 & 49 & 185 \\
\hline 23 & 218 & 49 & 227 \\
\hline 24 & 207 & 52 & 226 \\
\hline 25 & 200 & 54 & 163 \\
\hline 25 & 181 & 54 & 203 \\
\hline 26 & 184 & 55 & 218 \\
\hline 27 & 163 & 56 & 152 Lowest SFF \\
\hline 28 & 222 & 59 & 207 \\
\hline 28 & 214 & 60 & 167 \\
\hline 30 & 163 & 62 & 185 \\
\hline 31 & 207 & 63 & 203 \\
\hline 35 & 196 & 63 & 240 \\
\hline 35 & 196 & & \\
\hline 35 & 167 & & \\
\hline 36 & 218 & & \\
\hline 38 & 207 & & \\
\hline 39 & 229 & & \\
\hline
\end{tabular}




\section{Appendix J: Conversion of note names to numbers}

Each musical note was assigned a numerical value for the purposes of generating a graph. The notes were numbered in order from $G 2$ up to A6, a total of 51 values.

\begin{tabular}{|c|c|c|c|}
\hline Number & $\begin{array}{c}\text { Note name } \\
\mathrm{C}_{4}=\text { Middle } \mathrm{C}\end{array}$ & Number & $\begin{array}{c}\text { Note name } \\
\mathrm{C}_{4}=\text { Middle } \mathrm{C}\end{array}$ \\
\hline 1 & G2 & 27 & A4 \\
\hline 2 & G\#2 & 28 & A\#4 \\
\hline 3 & A2 & 29 & B4 \\
\hline 4 & A\#2 & 30 & C5 \\
\hline 5 & B2 & 31 & C\#5 \\
\hline 6 & C3 & 32 & D5 \\
\hline 7 & C\#3 & 33 & D\#5 \\
\hline 8 & D3 & 34 & E5 \\
\hline 9 & D\#3 & 35 & F5 \\
\hline 10 & E3 & 36 & F\#5 \\
\hline 11 & F3 & 37 & G5 \\
\hline 12 & F\#3 & 38 & $\mathrm{G} \# 5$ \\
\hline 13 & G3 & 39 & A5 \\
\hline 14 & G\#3 & 40 & A\#5 \\
\hline 15 & A3 & 41 & B5 \\
\hline 16 & A\#3 & 42 & C6 \\
\hline 17 & B3 & 43 & C\#6 \\
\hline 18 & C4 & 44 & D6 \\
\hline 19 & C\#4 & 45 & D\#6 \\
\hline 20 & D4 & 46 & E6 \\
\hline 21 & D\# & 47 & F6 \\
\hline 22 & E4 & 48 & F\#6 \\
\hline 23 & F4 & 49 & G6 \\
\hline 24 & F\#4 & 50 & G\#6 \\
\hline 25 & G4 & 51 & A6 \\
\hline 26 & G\# & & \\
\hline
\end{tabular}




\section{Appendix K: Repeated measurements raw data}

\section{Anthropometric and SFF repeated measurements}

\begin{tabular}{|c|c|c|c|c|c|c|c|c|}
\hline \multicolumn{3}{|c|}{ Neck circumference $(\mathrm{cm})$} & \multicolumn{3}{|c|}{ Neck length $(\mathrm{cm})$} & \multicolumn{3}{|c|}{$\begin{array}{l}\text { Speaking fundamental } \\
\text { frequency }(\mathrm{Hz})\end{array}$} \\
\hline $\begin{array}{l}\text { First } \\
\text { reading }\end{array}$ & $\begin{array}{l}\text { Second } \\
\text { reading }\end{array}$ & Difference & $\begin{array}{c}\text { First } \\
\text { reading }\end{array}$ & $\begin{array}{l}\text { Second } \\
\text { reading }\end{array}$ & Difference & $\begin{array}{c}\text { First } \\
\text { reading }\end{array}$ & $\begin{array}{l}\text { Second } \\
\text { reading }\end{array}$ & Difference \\
\hline 31.0 & 31.2 & 0.2 & 10.5 & 10.3 & 0.2 & 196 & 203 & 7 \\
\hline 34.0 & 34.0 & 0.0 & 11.8 & 12.1 & 0.3 & 195 & 203 & 8 \\
\hline 32.1 & 31.2 & 0.9 & 12.0 & 11.6 & 0.4 & 229 & 227 & 2 \\
\hline 32.8 & 32.4 & 0.4 & 12.4 & 12.5 & 0.1 & 196 & 191 & 5 \\
\hline 39.0 & 39.2 & 0.2 & 10.4 & 9.9 & 0.5 & 152 & 155 & 3 \\
\hline 35.0 & 34.4 & 0.6 & 13.9 & 13.5 & 0.4 & 184 & 179 & 5 \\
\hline 30.5 & 30.7 & 0.2 & 11.5 & 11.7 & 0.2 & 159 & 155 & 4 \\
\hline 32.5 & 31.8 & 0.7 & 11.5 & 11.5 & 0.0 & 207 & 203 & 4 \\
\hline 34.4 & 34.2 & 0.2 & 13.8 & 13.7 & 0.1 & 167 & 171 & 4 \\
\hline 45.4 & 45.5 & 0.1 & 12.6 & 12.5 & 0.1 & 167 & 167 & 0 \\
\hline 34.9 & 34.4 & 0.5 & 11.2 & 11.4 & 0.2 & 218 & 215 & 3 \\
\hline 31.0 & 30.9 & 0.1 & 14.6 & 14.5 & 0.1 & 203 & 203 & 0 \\
\hline 31.1 & 31.2 & 0.1 & 12.1 & 12.1 & 0.0 & 243 & 243 & 0 \\
\hline 38.0 & 38.8 & 0.8 & 9.0 & 9.3 & 0.3 & 240 & 239 & 1 \\
\hline
\end{tabular}


Laryngeal repeated measurements

\begin{tabular}{|c|c|c|c|c|c|c|c|c|}
\hline \multicolumn{3}{|c|}{ Cricoid cartilage $(\mathrm{mm})$} & \multicolumn{3}{|c|}{ Cricothyroid space (mm) } & \multicolumn{3}{|c|}{ Thyroid cartilage $(\mathrm{mm})$} \\
\hline $\begin{array}{l}\text { First } \\
\text { reading }\end{array}$ & $\begin{array}{l}\text { Second } \\
\text { reading }\end{array}$ & Difference & $\begin{array}{l}\text { First } \\
\text { reading }\end{array}$ & $\begin{array}{l}\text { Second } \\
\text { reading }\end{array}$ & Difference & $\begin{array}{c}\text { First } \\
\text { reading }\end{array}$ & $\begin{array}{l}\text { Second } \\
\text { reading }\end{array}$ & Difference \\
\hline 4.8 & 5.1 & 0.3 & 9.9 & 10.1 & 0.2 & 13.1 & 13.2 & 0.1 \\
\hline 4.8 & 4.4 & 0.4 & 10.6 & 11.4 & 0.8 & 11.1 & 10.9 & 0.2 \\
\hline 5.8 & 5.4 & 0.4 & 10.0 & 10.3 & 0.3 & 14.8 & 14.3 & 0.5 \\
\hline 5.4 & 5.3 & 0.1 & 12.3 & 12.2 & 0.1 & 14.6 & 14.6 & 0.0 \\
\hline 5.4 & 4.9 & 0.5 & 10.8 & 10.9 & 0.1 & 13.8 & 13.8 & 0.0 \\
\hline 7.2 & 7.1 & 0.1 & 10.5 & 10.5 & 0.0 & 12.2 & 12.0 & 0.2 \\
\hline 5.7 & 5.2 & 0.5 & 11.4 & 11.9 & 0.5 & 11.2 & 10.4 & 0.8 \\
\hline 5.4 & 4.8 & 0.6 & 7.3 & 7.5 & 0.2 & 13.3 & 13.1 & 0.2 \\
\hline 5.7 & 5.4 & 0.3 & 12.0 & 13.1 & 1.1 & 10.5 & 10.2 & 0.3 \\
\hline 5.8 & 5.5 & 0.3 & 10.2 & 10.5 & 0.3 & 12.8 & 12.8 & 0.0 \\
\hline 6.4 & 6.4 & 0.0 & 11.1 & 10.6 & 0.5 & 9.5 & 9.5 & 0.0 \\
\hline 6.8 & 6.8 & 0.0 & 12.4 & 13.3 & 0.9 & 14.5 & 13.5 & 1.0 \\
\hline 5.4 & 5.7 & 0.3 & 9.4 & 9.4 & 0.0 & 14.7 & 14.4 & 0.3 \\
\hline 4.5 & 4.4 & 0.1 & 7.3 & 7.4 & 0.1 & 11.4 & 11.1 & 0.3 \\
\hline
\end{tabular}


Appendix L: Relationship between practice and performance vocal range

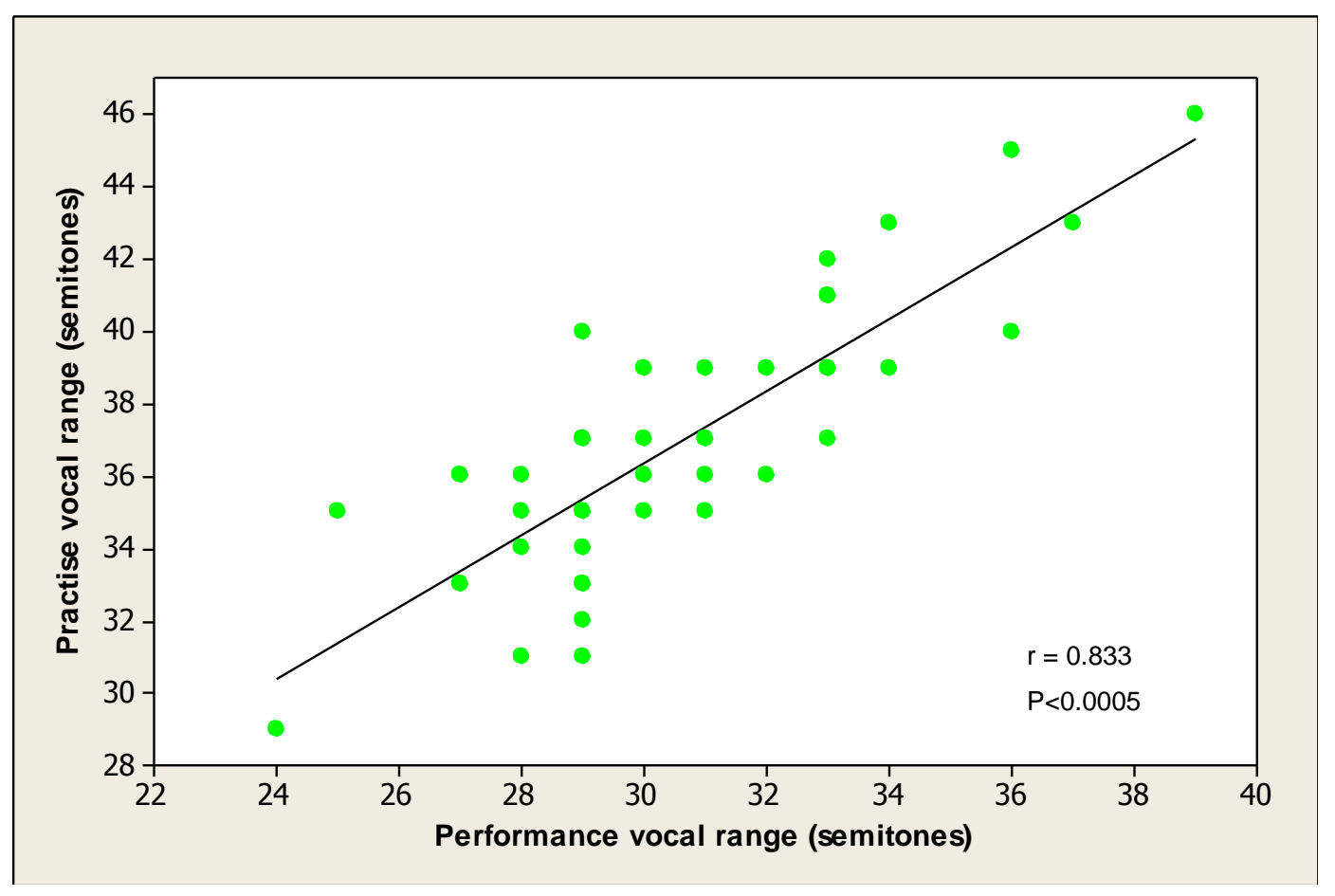




\section{Appendix M: Correlations of all data}

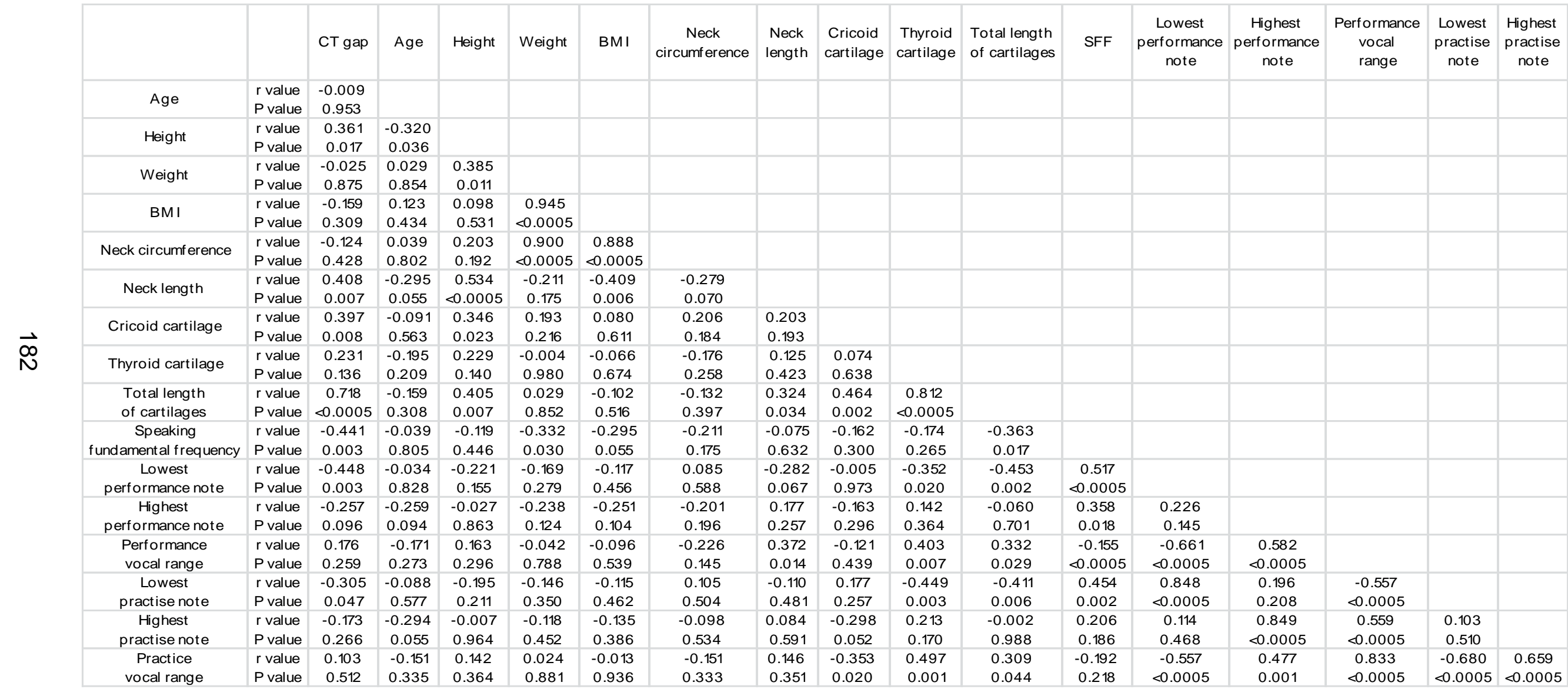




\section{Appendix N: Soprano correlations}

\begin{tabular}{|c|c|c|c|c|c|c|c|c|c|c|c|c|c|c|c|c|c|}
\hline & & CT gap & Age & Height & Weight & BMI & $\begin{array}{c}\text { Neck } \\
\text { circumf erence }\end{array}$ & $\begin{array}{l}\text { Neck } \\
\text { length }\end{array}$ & $\begin{array}{l}\text { Cricoid } \\
\text { cartilage }\end{array}$ & $\begin{array}{l}\text { Thyroid } \\
\text { cartilage }\end{array}$ & $\begin{array}{l}\text { Total length } \\
\text { of cartilages }\end{array}$ & SFF & $\begin{array}{l}\text { Lowest } \\
\text { performance } \\
\text { note }\end{array}$ & $\begin{array}{l}\text { Highest } \\
\text { performance } \\
\text { note }\end{array}$ & $\begin{array}{l}\text { Performance } \\
\text { vocal } \\
\text { range }\end{array}$ & $\begin{array}{c}\text { Lowest } \\
\text { practise } \\
\text { note }\end{array}$ & $\begin{array}{l}\text { Highest } \\
\text { practise } \\
\text { note }\end{array}$ \\
\hline Age & $\begin{array}{l}r \text { value } \\
P \text { value }\end{array}$ & $\begin{array}{l}-0.119 \\
0.518\end{array}$ & & & & & & & & & & & & & & & \\
\hline Height & $\begin{array}{l}r \text { value } \\
P \text { value }\end{array}$ & $\begin{array}{l}0.220 \\
0.226\end{array}$ & $\begin{array}{l}-0.397 \\
0.024\end{array}$ & & & & & & & & & & & & & & \\
\hline Weight & $\begin{array}{l}r \text { value } \\
P \text { value }\end{array}$ & $\begin{array}{l}-0.412 \\
0.438\end{array}$ & $\begin{array}{r}-0.036 \\
0.847\end{array}$ & $\begin{array}{l}0.391 \\
0.027\end{array}$ & & & & & & & & & & & & & \\
\hline BMI & $\begin{array}{l}r \text { value } \\
P \text { value }\end{array}$ & $\begin{array}{c}-0.238 \\
0.190\end{array}$ & $\begin{array}{l}0.070 \\
0.705\end{array}$ & $\begin{array}{l}0.132 \\
0.472\end{array}$ & $\begin{array}{c}0.950 \\
<0.0005\end{array}$ & & & & & & & & & & & & \\
\hline Neck circumference & $\begin{array}{l}r \text { value } \\
P \text { value }\end{array}$ & $\begin{array}{l}-0.169 \\
0.356\end{array}$ & $\begin{array}{l}-0.007 \\
0.970\end{array}$ & $\begin{array}{l}0.204 \\
0.263\end{array}$ & $\begin{array}{c}0.930 \\
<0.0005\end{array}$ & $\begin{array}{c}0.919 \\
<0.0005\end{array}$ & & & & & & & & & & & \\
\hline Neck length & $\begin{array}{l}r \text { value } \\
P \text { value }\end{array}$ & $\begin{array}{l}0.421 \\
0.016\end{array}$ & $\begin{array}{r}-0.368 \\
0.038\end{array}$ & $\begin{array}{l}0.545 \\
0.001\end{array}$ & $\begin{array}{c}-0.240 \\
0.186 \\
\end{array}$ & $\begin{array}{l}-0.437 \\
0.012 \\
\end{array}$ & $\begin{array}{l}-0.331 \\
0.064\end{array}$ & & & & & & & & & & \\
\hline Cricoid cartilage & $\begin{array}{l}r \text { value } \\
P \text { value }\end{array}$ & $\begin{array}{l}0.334 \\
0.061\end{array}$ & $\begin{array}{l}-0.144 \\
0.432\end{array}$ & $\begin{array}{l}0.290 \\
0.108\end{array}$ & $\begin{array}{l}0.116 \\
0.526\end{array}$ & $\begin{array}{r}0.017 \\
0.928\end{array}$ & $\begin{array}{l}0.128 \\
0.484\end{array}$ & $\begin{array}{r}0.195 \\
0.286\end{array}$ & & & & & & & & & \\
\hline Thyroid cartilage & $\begin{array}{l}r \text { value } \\
P \text { value }\end{array}$ & $\begin{array}{l}0.212 \\
0.243\end{array}$ & $\begin{array}{l}-0.143 \\
0.435\end{array}$ & $\begin{array}{l}0.141 \\
0.442\end{array}$ & $\begin{array}{l}-0.126 \\
0.493\end{array}$ & $\begin{array}{l}-0.163 \\
0.372\end{array}$ & $\begin{array}{r}-0.250 \\
0.167\end{array}$ & $\begin{array}{r}0.239 \\
0.187\end{array}$ & $\begin{array}{l}0.117 \\
0.524\end{array}$ & & & & & & & & \\
\hline $\begin{array}{l}\text { Total length } \\
\text { of cartilages }\end{array}$ & $\begin{array}{l}r \text { value } \\
P \text { value }\end{array}$ & $\begin{array}{c}0.396 \\
<0.0005\end{array}$ & $\begin{array}{l}-0.187 \\
0.304\end{array}$ & $\begin{array}{l}0.237 \\
0.139\end{array}$ & $\begin{array}{l}-0.127 \\
0.487\end{array}$ & $\begin{array}{l}-0.221 \\
0.223\end{array}$ & $\begin{array}{l}-0.223 \\
0.219\end{array}$ & $\begin{array}{l}0.409 \\
0.020\end{array}$ & $\begin{array}{l}0.467 \\
0.007\end{array}$ & $\begin{array}{c}0.818 \\
<0.0005\end{array}$ & & & & & & & \\
\hline $\begin{array}{l}\text { Speaking } \\
\text { fundamental frequency }\end{array}$ & $\begin{array}{l}r \text { value } \\
P \text { value }\end{array}$ & $\begin{array}{c}-0.347 \\
0.052\end{array}$ & $\begin{array}{l}0.109 \\
0.552\end{array}$ & $\begin{array}{l}-0.017 \\
0.927\end{array}$ & $\begin{array}{l}-0.311 \\
0.083\end{array}$ & $\begin{array}{c}-0.290 \\
0.108\end{array}$ & $\begin{array}{c}-0.332 \\
0.064\end{array}$ & $\begin{array}{l}-0.050 \\
0.785\end{array}$ & $\begin{array}{l}-0.236 \\
0.194 \\
\end{array}$ & $\begin{array}{l}-0.111 \\
0.547\end{array}$ & $\begin{array}{c}-0.294 \\
0.102\end{array}$ & & & & & & \\
\hline $\begin{array}{l}\text { Lowest } \\
\text { performance note }\end{array}$ & $\begin{array}{l}r \text { value } \\
P \text { value }\end{array}$ & $\begin{array}{c}-0.424 \\
0.015\end{array}$ & $\begin{array}{l}0.170 \\
0.353\end{array}$ & $\begin{array}{c}-0.322 \\
0.072\end{array}$ & $\begin{array}{l}-0.121 \\
0.511\end{array}$ & $\begin{array}{c}-0.048 \\
0.795\end{array}$ & $\begin{array}{l}0.029 \\
0.875\end{array}$ & $\begin{array}{l}-0.419 \\
0.017\end{array}$ & $\begin{array}{l}0.005 \\
0.979\end{array}$ & $\begin{array}{l}-0.538 \\
0.002\end{array}$ & $\begin{array}{l}-0.271 \\
0.001\end{array}$ & $\begin{array}{l}0.414 \\
0.018\end{array}$ & & & & & \\
\hline $\begin{array}{c}\text { Highest } \\
\text { performance note }\end{array}$ & $\begin{array}{l}r \text { value } \\
P \text { value }\end{array}$ & $\begin{array}{l}0.016 \\
0.931\end{array}$ & $\begin{array}{l}-0.200 \\
0.272\end{array}$ & $\begin{array}{l}0.081 \\
0.661\end{array}$ & $\begin{array}{l}-0.327 \\
0.068\end{array}$ & $\begin{array}{l}-0.375 \\
0.034\end{array}$ & $\begin{array}{l}-0.353 \\
0.048\end{array}$ & $\begin{array}{l}0.382 \\
0.031\end{array}$ & $\begin{array}{c}-0.076 \\
0.679\end{array}$ & $\begin{array}{l}0.306 \\
0.088\end{array}$ & $\begin{array}{l}0.202 \\
0.268\end{array}$ & $\begin{array}{l}0.078 \\
0.673\end{array}$ & $\begin{array}{l}-0.208 \\
0.252\end{array}$ & & & & \\
\hline $\begin{array}{l}\text { Performance } \\
\text { vocal range }\end{array}$ & $\begin{array}{l}r \text { value } \\
P \text { value }\end{array}$ & $\begin{array}{r}0.292 \\
0.105\end{array}$ & $\begin{array}{l}-0.237 \\
0.191\end{array}$ & $\begin{array}{l}0.264 \\
0.144\end{array}$ & $\begin{array}{l}-0.123 \\
0.504\end{array}$ & $\begin{array}{l}-0.021 \\
0.269\end{array}$ & $\begin{array}{l}-0.238 \\
0.189\end{array}$ & $\begin{array}{r}0.516 \\
0.003\end{array}$ & $\begin{array}{l}-0.050 \\
0.784\end{array}$ & $\begin{array}{l}0.548 \\
0.001\end{array}$ & $\begin{array}{l}0.505 \\
0.003\end{array}$ & $\begin{array}{l}-0.227 \\
0.211\end{array}$ & $\begin{array}{l}-0.794 \\
<0.0005\end{array}$ & $\begin{array}{c}0.760 \\
<0.0005\end{array}$ & & & \\
\hline $\begin{array}{l}\text { Lowest } \\
\text { practise note }\end{array}$ & $\begin{array}{l}r \text { value } \\
P \text { value }\end{array}$ & $\begin{array}{l}-0.192 \\
0.292\end{array}$ & $\begin{array}{l}0.008 \\
0.964\end{array}$ & $\begin{array}{c}-0.185 \\
0.311\end{array}$ & $\begin{array}{l}-0.063 \\
0.732\end{array}$ & $\begin{array}{c}-0.048 \\
0.795\end{array}$ & $\begin{array}{l}0.077 \\
0.675\end{array}$ & $\begin{array}{l}-0.169 \\
0.356\end{array}$ & $\begin{array}{l}0.226 \\
0.214\end{array}$ & $\begin{array}{l}-0.485 \\
0.005\end{array}$ & $\begin{array}{r}-0.374 \\
0.035\end{array}$ & $\begin{array}{c}0.249 \\
0.170\end{array}$ & $\begin{array}{l}0.782 \\
<0.005\end{array}$ & $\begin{array}{l}-0.203 \\
0.266\end{array}$ & $\begin{array}{l}-0.646 \\
<0.0005\end{array}$ & & \\
\hline $\begin{array}{c}\text { Highest } \\
\text { practise note }\end{array}$ & $\begin{array}{l}r \text { value } \\
P \text { value }\end{array}$ & $\begin{array}{l}0.009 \\
0.963\end{array}$ & $\begin{array}{l}-0.285 \\
0.114\end{array}$ & $\begin{array}{l}0.021 \\
0.907\end{array}$ & $\begin{array}{l}-0.212 \\
0.244\end{array}$ & $\begin{array}{c}-0.242 \\
0.182\end{array}$ & $\begin{array}{l}-0.177 \\
0.334\end{array}$ & $\begin{array}{l}0.277 \\
0.125\end{array}$ & $\begin{array}{l}-0.283 \\
0.116\end{array}$ & $\begin{array}{c}0.242 \\
0.181\end{array}$ & $\begin{array}{l}0.107 \\
0.560\end{array}$ & $\begin{array}{l}-0.033 \\
0.856\end{array}$ & $\begin{array}{l}-0.228 \\
0.210\end{array}$ & $\begin{array}{c}0.777 \\
<0.0005\end{array}$ & $\begin{array}{c}0.634 \\
<0.0005\end{array}$ & $\begin{array}{l}-0.152 \\
0.407\end{array}$ & \\
\hline $\begin{array}{c}\text { Practice } \\
\text { vocal range }\end{array}$ & $\begin{array}{l}r \text { value } \\
P \text { value }\end{array}$ & $\begin{array}{l}0.137 \\
0.454\end{array}$ & $\begin{array}{l}-0.186 \\
0.309\end{array}$ & $\begin{array}{l}0.140 \\
0.444\end{array}$ & $\begin{array}{c}-0.090 \\
0.623\end{array}$ & $\begin{array}{l}-0.120 \\
0.514\end{array}$ & $\begin{array}{l}-0.164 \\
0.369\end{array}$ & $\begin{array}{r}0.290 \\
0.107\end{array}$ & $\begin{array}{l}-0.333 \\
0.062\end{array}$ & $\begin{array}{l}0.486 \\
0.005\end{array}$ & $\begin{array}{l}0.324 \\
0.070\end{array}$ & $\begin{array}{l}-0.192 \\
0.293\end{array}$ & $\begin{array}{c}-0.680 \\
<0.0005\end{array}$ & $\begin{array}{c}0.629 \\
<0.0005\end{array}$ & $\begin{array}{c}0.843 \\
<0.0005\end{array}$ & $\begin{array}{l}-0.782 \\
<0.0005\end{array}$ & $\begin{array}{c}0.735 \\
<0.0005\end{array}$ \\
\hline
\end{tabular}




\section{Appendix 0: Mezzo-soprano correlations}

\begin{tabular}{|c|c|c|c|c|c|c|c|c|c|c|c|c|c|c|c|c|c|}
\hline & & CT gap & Age & Height & Weight & BMI & $\begin{array}{c}\text { Neck } \\
\text { circumference }\end{array}$ & $\begin{array}{l}\text { Neck } \\
\text { length }\end{array}$ & $\begin{array}{l}\text { Cricoid } \\
\text { cartilage }\end{array}$ & $\begin{array}{l}\text { Thyroid } \\
\text { cartilage }\end{array}$ & $\begin{array}{l}\text { Total length } \\
\text { of cartilages }\end{array}$ & SFF & $\begin{array}{l}\text { Lowest } \\
\text { performance } \\
\text { note }\end{array}$ & $\begin{array}{c}\text { Highest } \\
\text { performance } \\
\text { note }\end{array}$ & $\begin{array}{l}\text { Performance } \\
\text { vocal } \\
\text { range }\end{array}$ & $\begin{array}{l}\text { Lowest } \\
\text { practise } \\
\text { note }\end{array}$ & $\begin{array}{c}\text { Highest } \\
\text { practise } \\
\text { note }\end{array}$ \\
\hline Age & $\begin{array}{l}\mathrm{r} \text { value } \\
\mathrm{P} \text { value }\end{array}$ & $\begin{array}{r}-0.027 \\
0.937\end{array}$ & & & & & & & & & & & & & & & \\
\hline Height & $\begin{array}{l}\mathrm{r} \text { value } \\
\mathrm{P} \text { value }\end{array}$ & $\begin{array}{l}0.645 \\
0.032\end{array}$ & $\begin{array}{r}-0.347 \\
0.295\end{array}$ & & & & & & & & & & & & & & \\
\hline Weight & $\begin{array}{l}\mathrm{r} \text { value } \\
\mathrm{P} \text { value }\end{array}$ & $\begin{array}{l}0.245 \\
0.468\end{array}$ & $\begin{array}{l}0.167 \\
0.624\end{array}$ & $\begin{array}{l}0.318 \\
0.340\end{array}$ & & & & & & & & & & & & & \\
\hline BMI & $\begin{array}{l}\mathrm{r} \text { value } \\
P \text { value }\end{array}$ & $\begin{array}{l}0.020 \\
0.952\end{array}$ & $\begin{array}{l}0.289 \\
0.389\end{array}$ & $\begin{array}{l}-0.035 \\
0.920\end{array}$ & $\begin{aligned} & 0.935 \\
&<0.0005\end{aligned}$ & & & & & & & & & & & & \\
\hline Neck circumf erence & $\begin{array}{l}\mathrm{r} \text { value } \\
\mathrm{P} \text { value }\end{array}$ & $\begin{array}{l}0.035 \\
0.918\end{array}$ & $\begin{array}{l}0.238 \\
0.481\end{array}$ & $\begin{array}{l}0.229 \\
0.497\end{array}$ & $\begin{array}{l}0.804 \\
0.003\end{array}$ & $\begin{array}{l}0.767 \\
0.006\end{array}$ & & & & & & & & & & & \\
\hline Neck length & $\begin{array}{l}\mathrm{r} \text { value } \\
\mathrm{P} \text { value }\end{array}$ & $\begin{array}{r}-0.060 \\
0.862\end{array}$ & $\begin{array}{r}-0.360 \\
0.277\end{array}$ & $\begin{array}{l}0.392 \\
0.233\end{array}$ & $\begin{array}{l}-0.271 \\
0.421\end{array}$ & $\begin{array}{c}-0.430 \\
0.187\end{array}$ & $\begin{array}{c}-0.009 \\
0.980\end{array}$ & & & & & & & & & & \\
\hline Cricoid cartilage & $\begin{array}{l}r \text { value } \\
P \text { value }\end{array}$ & $\begin{array}{l}0.427 \\
0.190\end{array}$ & $\begin{array}{c}-0.131 \\
0.701\end{array}$ & $\begin{array}{l}0.389 \\
0.238\end{array}$ & $\begin{array}{l}0.372 \\
0.259\end{array}$ & $\begin{array}{l}0.254 \\
0.452\end{array}$ & $\begin{array}{l}0.517 \\
0.103\end{array}$ & $\begin{array}{l}0.031 \\
0.927\end{array}$ & & & & & & & & & \\
\hline Thyroid cartilage & $\begin{array}{l}\mathrm{r} \text { value } \\
\mathrm{P} \text { value }\end{array}$ & $\begin{array}{l}0.485 \\
0.130\end{array}$ & $\begin{array}{c}-0.328 \\
0.324\end{array}$ & $\begin{array}{l}0.525 \\
0.097\end{array}$ & $\begin{array}{l}0.382 \\
0.246\end{array}$ & $\begin{array}{l}0.226 \\
0.505\end{array}$ & $\begin{array}{l}0.065 \\
0.849\end{array}$ & $\begin{array}{r}-0.288 \\
0.391\end{array}$ & $\begin{array}{l}0.007 \\
0.985\end{array}$ & & & & & & & & \\
\hline $\begin{array}{l}\text { Total length } \\
\text { of cartilages }\end{array}$ & $\begin{array}{l}r \text { value } \\
P \text { value }\end{array}$ & $\begin{array}{l}0.792 \\
0.004\end{array}$ & $\begin{array}{l}-0.275 \\
0.413\end{array}$ & $\begin{array}{l}0.689 \\
0.019\end{array}$ & $\begin{array}{l}0.443 \\
0.172\end{array}$ & $\begin{array}{l}0.226 \\
0.504\end{array}$ & $\begin{array}{r}0.174 \\
0.609\end{array}$ & $\begin{array}{l}-0.221 \\
0.513\end{array}$ & $\begin{array}{l}0.375 \\
0.255\end{array}$ & $\begin{array}{c}0.891 \\
<0.0005\end{array}$ & & & & & & & \\
\hline $\begin{array}{c}\text { Speaking } \\
\text { fundamental frequency }\end{array}$ & $\begin{array}{l}r \text { value } \\
P \text { value }\end{array}$ & $\begin{array}{l}-0.265 \\
0.430\end{array}$ & $\begin{array}{l}-0.057 \\
0.869\end{array}$ & $\begin{array}{l}-0.037 \\
0.914\end{array}$ & $\begin{array}{c}-0.365 \\
0.270\end{array}$ & $\begin{array}{l}-0.385 \\
0.243\end{array}$ & $\begin{array}{l}0.072 \\
0.833\end{array}$ & $\begin{array}{l}0.433 \\
0.183\end{array}$ & $\begin{array}{l}0.324 \\
0.332\end{array}$ & $\begin{array}{c}-0.486 \\
0.130\end{array}$ & $\begin{array}{l}-0.370 \\
0.263\end{array}$ & & & & & & \\
\hline $\begin{array}{l}\text { Lowest } \\
\text { performance note }\end{array}$ & $\begin{array}{l}\mathrm{r} \text { value } \\
\mathrm{P} \text { value }\end{array}$ & $\begin{array}{l}-0.107 \\
0.754\end{array}$ & $\begin{array}{l}-0.137 \\
0.687\end{array}$ & $\begin{array}{l}0.253 \\
0.453\end{array}$ & $\begin{array}{r}-0.192 \\
0.571\end{array}$ & $\begin{array}{l}-0.272 \\
0.418\end{array}$ & $\begin{array}{l}0.288 \\
0.391\end{array}$ & $\begin{array}{l}0.536 \\
0.089\end{array}$ & $\begin{array}{c}0.15 \\
0.345\end{array}$ & $\begin{array}{c}-0.200 \\
0.517\end{array}$ & $\begin{array}{c}-0.125 \\
0.714\end{array}$ & $\begin{array}{l}0.337 \\
0.310\end{array}$ & & & & & \\
\hline $\begin{array}{l}\text { Highest } \\
\text { performance note }\end{array}$ & $\begin{array}{l}\mathrm{r} \text { value } \\
\mathrm{P} \text { value }\end{array}$ & $\begin{array}{l}-0.221 \\
0.513\end{array}$ & $\begin{array}{l}-0.047 \\
0.890\end{array}$ & $\begin{array}{l}0.326 \\
0.328\end{array}$ & $\begin{array}{l}0.142 \\
0.677\end{array}$ & $\begin{array}{l}0.012 \\
0.972\end{array}$ & $\begin{array}{l}0.134 \\
0.695\end{array}$ & $\begin{array}{l}0.636 \\
0.035\end{array}$ & $\begin{array}{l}0.036 \\
0.916\end{array}$ & $\begin{array}{l}-0.331 \\
0.320\end{array}$ & $\begin{array}{c}-0.307 \\
0.358\end{array}$ & $\begin{array}{l}0.217 \\
0.521\end{array}$ & $\begin{array}{l}0.180 \\
0.597\end{array}$ & & & & \\
\hline $\begin{array}{l}\text { Performance } \\
\text { vocal range }\end{array}$ & $\begin{array}{l}\mathrm{r} \text { value } \\
\mathrm{P} \text { value }\end{array}$ & $\begin{array}{c}-0.029 \\
0.932\end{array}$ & $\begin{array}{l}0.100 \\
0.770\end{array}$ & $\begin{array}{c}-0.045 \\
0.895\end{array}$ & $\begin{array}{l}0.260 \\
0.439\end{array}$ & $\begin{array}{l}0.259 \\
0.442\end{array}$ & $\begin{array}{c}-0.189 \\
0.578\end{array}$ & $\begin{array}{l}-0.127 \\
0.709\end{array}$ & $\begin{array}{l}-0.271 \\
0.421\end{array}$ & $\begin{array}{l}0.012 \\
0.973\end{array}$ & $\begin{array}{c}-0.062 \\
0.856\end{array}$ & $\begin{array}{l}-0.186 \\
0.583\end{array}$ & $\begin{array}{r}-0.822 \\
0.002\end{array}$ & $\begin{array}{l}0.413 \\
0.207\end{array}$ & & & \\
\hline $\begin{array}{l}\text { Lowest } \\
\text { practise note }\end{array}$ & $\begin{array}{l}r \text { value } \\
P \text { value }\end{array}$ & $\begin{array}{l}-0.161 \\
0.635\end{array}$ & $\begin{array}{l}-0.036 \\
0.916\end{array}$ & $\begin{array}{l}0.031 \\
0.927\end{array}$ & $\begin{array}{c}-0.266 \\
0.429\end{array}$ & $\begin{array}{l}-0.277 \\
0.409\end{array}$ & $\begin{array}{l}0.233 \\
0.490\end{array}$ & $\begin{array}{l}0.589 \\
0.057\end{array}$ & $\begin{array}{l}0.429 \\
0.188\end{array}$ & $\begin{array}{l}-0.569 \\
0.068\end{array}$ & $\begin{array}{l}-0.370 \\
0.262\end{array}$ & $\begin{array}{l}0.525 \\
0.097\end{array}$ & $\begin{array}{l}0.880 \\
<0.0005\end{array}$ & $\begin{array}{l}0.295 \\
0.378\end{array}$ & $\begin{array}{c}-0.644 \\
0.033\end{array}$ & & \\
\hline $\begin{array}{c}\text { Highest } \\
\text { practise note }\end{array}$ & $\begin{array}{l}\mathrm{r} \text { value } \\
P \text { value }\end{array}$ & $\begin{array}{r}0.334 \\
0.315\end{array}$ & $\begin{array}{l}0.034 \\
0.921\end{array}$ & $\begin{array}{l}0.580 \\
0.061\end{array}$ & $\begin{array}{l}0.418 \\
0.201\end{array}$ & $\begin{array}{l}0.207 \\
0.541\end{array}$ & $\begin{array}{l}0.092 \\
0.789\end{array}$ & $\begin{array}{l}0.355 \\
0.284\end{array}$ & $\begin{array}{r}-0.046 \\
0.893\end{array}$ & $\begin{array}{l}0.193 \\
0.569\end{array}$ & $\begin{array}{l}0.245 \\
0.468\end{array}$ & $\begin{array}{c}-0.252 \\
0.455\end{array}$ & $\begin{array}{l}-0.261 \\
0.438\end{array}$ & $\begin{array}{l}0.712 \\
0.014\end{array}$ & $\begin{array}{l}0.655 \\
0.029\end{array}$ & $\begin{array}{c}-0.250 \\
0.458\end{array}$ & \\
\hline $\begin{array}{c}\text { Practice } \\
\text { vocal range }\end{array}$ & $\begin{array}{l}r \text { value } \\
P \text { value }\end{array}$ & $\begin{array}{l}0.292 \\
0.393\end{array}$ & $\begin{array}{l}0.044 \\
0.897\end{array}$ & $\begin{array}{l}0.278 \\
0.408\end{array}$ & $\begin{array}{l}0.412 \\
0.207\end{array}$ & $\begin{array}{r}0.311 \\
0.352\end{array}$ & $\begin{array}{c}-0.124 \\
0.717\end{array}$ & $\begin{array}{c}-0.248 \\
0.463\end{array}$ & $\begin{array}{c}-0.339 \\
0.308\end{array}$ & $\begin{array}{l}0.518 \\
0.103\end{array}$ & $\begin{array}{l}0.399 \\
0.224\end{array}$ & $\begin{array}{c}-0.516 \\
0.104\end{array}$ & $\begin{array}{c}-0.782 \\
0.004\end{array}$ & $\begin{array}{l}0.153 \\
0.653\end{array}$ & $\begin{array}{l}0.813 \\
0.002\end{array}$ & $\begin{array}{r}-0.864 \\
0.001\end{array}$ & $\begin{array}{l}0.703 \\
0.016\end{array}$ \\
\hline
\end{tabular}


Appendix P: Data summary of CT space measurements $(\mathrm{mm})$ with vocal range in semitones, lowest and highest performance notes and Fach

\begin{tabular}{|c|c|c|c|c|}
\hline $\begin{array}{c}\text { Anterior } \\
\text { cricothyroid } \\
\text { space }(\mathrm{mm})\end{array}$ & $\begin{array}{l}\text { Vocal } \\
\text { range } \\
\text { (semitones) }\end{array}$ & $\begin{array}{c}\text { Lowest } \\
\text { performance } \\
\text { note } \\
\text { (pitch name) }\end{array}$ & $\begin{array}{c}\text { Highest } \\
\text { performance } \\
\text { note } \\
\text { (pitch name) }\end{array}$ & Fach \\
\hline 7.3 & 33 & G3 & E6 & Soubrette \\
\hline 7.3 & 29 & A3 & D6 & Spinto \\
\hline 8.5 & 29 & A3 & D6 & Light lyric \\
\hline 8.5 & 30 & A\#3 & E6 & Coloratura \\
\hline 9.0 & 29 & G3 & $\mathrm{C6}$ & Light lyric \\
\hline 9.2 & 29 & A3 & D6 & Light lyric \\
\hline 9.3 & 28 & $\mathrm{G} \# 3$ & C6 & Soubrette \\
\hline 9.4 & 30 & A3 & D\#6 & Coloratura \\
\hline 9.9 & 29 & A3 & D6 & Coloratura \\
\hline 10.0 & 31 & G3 & D6 & Light lyric \\
\hline 10.0 & 29 & G3 & $\mathrm{C} 6$ & Light lyric \\
\hline 10.1 & 32 & G3 & D\#6 & Heavy lyric \\
\hline 10.2 & 31 & F3 & C6 & Dramatic \\
\hline 10.2 & 33 & F\#3 & D\#6 & Soubrette \\
\hline 10.2 & 30 & G3 & C\#6 & Spinto \\
\hline 10.5 & 33 & F3 & D6 & Spinto \\
\hline 10.5 & 27 & G3 & A\#5 & Dramatic \\
\hline 10.5 & 32 & E3 & C6 & Mezzo \\
\hline 10.6 & 29 & F3 & A\#5 & Mezzo \\
\hline 10.6 & 28 & E3 & G\#5 & Mezzo \\
\hline 10.8 & 34 & E3 & D6 & Heavy Lyric \\
\hline 10.9 & 24 & A\#3 & A\#5 & Light lyric \\
\hline 10.9 & 29 & A3 & D6 & Soubrette \\
\hline 11.1 & 28 & A3 & C\#6 & Dramatic \\
\hline 11.1 & 36 & C3 & C6 & Mezzo \\
\hline 11.1 & 27 & G3 & A\#5 & Mezzo \\
\hline 11.2 & 31 & G3 & D6 & Heavy lyric \\
\hline 11.2 & 27 & A3 & C6 & Light Lyric \\
\hline 11.3 & 37 & D3 & D\#6 & Light Lyric \\
\hline 11.4 & 33 & C3 & A5 & Mezzo \\
\hline 11.4 & 27 & A3 & C6 & Spinto \\
\hline 11.6 & 29 & G3 & C6 & Heavy lyric \\
\hline 11.7 & 31 & G3 & D6 & Light lyric \\
\hline 11.7 & 29 & F\#3 & B5 & Mezzo \\
\hline 11.7 & 28 & G3 & B5 & Mezzo \\
\hline 11.9 & 30 & E3 & A\#5 & Spinto \\
\hline 12.0 & 31 & G3 & D6 & Mezzo \\
\hline 12.0 & 36 & F3 & F6 & Heavy lyric \\
\hline 12.3 & 39 & D3 & F6 & Light lyric \\
\hline 12.4 & 34 & G3 & F6 & Coloratura \\
\hline 12.4 & 33 & G3 & E6 & Spinto \\
\hline 13.0 & 25 & G3 & G\#5 & Mezzo \\
\hline 14.0 & 33 & C3 & A5 & Mezzo \\
\hline
\end{tabular}


Appendix Q: Data comparing age to performance vocal range

\begin{tabular}{|c|c|c|c|}
\hline Age (years) & $\begin{array}{l}\text { Performance } \\
\text { vocal range } \\
\text { (semitones) }\end{array}$ & Age (years) & $\begin{array}{l}\text { Performance } \\
\text { vocal range } \\
\text { (semitones) }\end{array}$ \\
\hline 18 & 31 & 40 & 30 \\
\hline 19 & 34 & 40 & 27 \\
\hline 20 & 32 & 41 & 30 \\
\hline 21 & 31 & 45 & 28 \\
\hline 21 & 30 & 48 & 31 \\
\hline 21 & 29 & 49 & 30 \\
\hline 21 & 29 & 49 & 33 \\
\hline 22 & 28 & 49 & 29 \\
\hline 23 & 36 & 49 & 37 \\
\hline 24 & 33 & 52 & 31 \\
\hline 25 & 33 & 54 & 33 \\
\hline 25 & 29 & 54 & 29 \\
\hline 26 & 33 & 55 & 27 \\
\hline 27 & 25 & 56 & 34 \\
\hline 28 & 29 & 59 & 29 \\
\hline 28 & 27 & 60 & 31 \\
\hline 30 & 36 & 62 & 28 \\
\hline 31 & 29 & 63 & $\begin{array}{l}24 \text { Smallest } \\
\text { vocal range }\end{array}$ \\
\hline 35 & $\begin{array}{l}39 \text { Largest } \\
\text { vocal range }\end{array}$ & 63 & 29 \\
\hline 35 & 29 & & \\
\hline 35 & 33 & & \\
\hline 36 & 28 & & \\
\hline 38 & 32 & & \\
\hline 39 & 27 & & \\
\hline
\end{tabular}

